The University of Chicago Science Series

\title{
PROTOPLASMIC ACTION AND NERVOUS ACTION
}

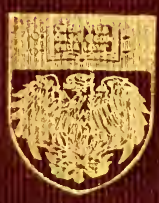

RALPH S. LILLIE 


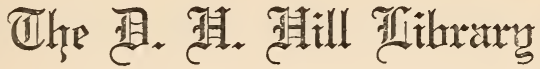

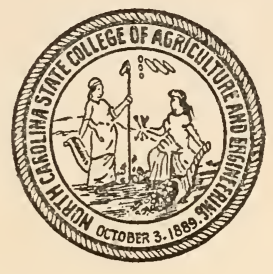

Narth Uaralina State Uallege

$$
\text { QH331 }
$$

L55 


\section{Date Due}

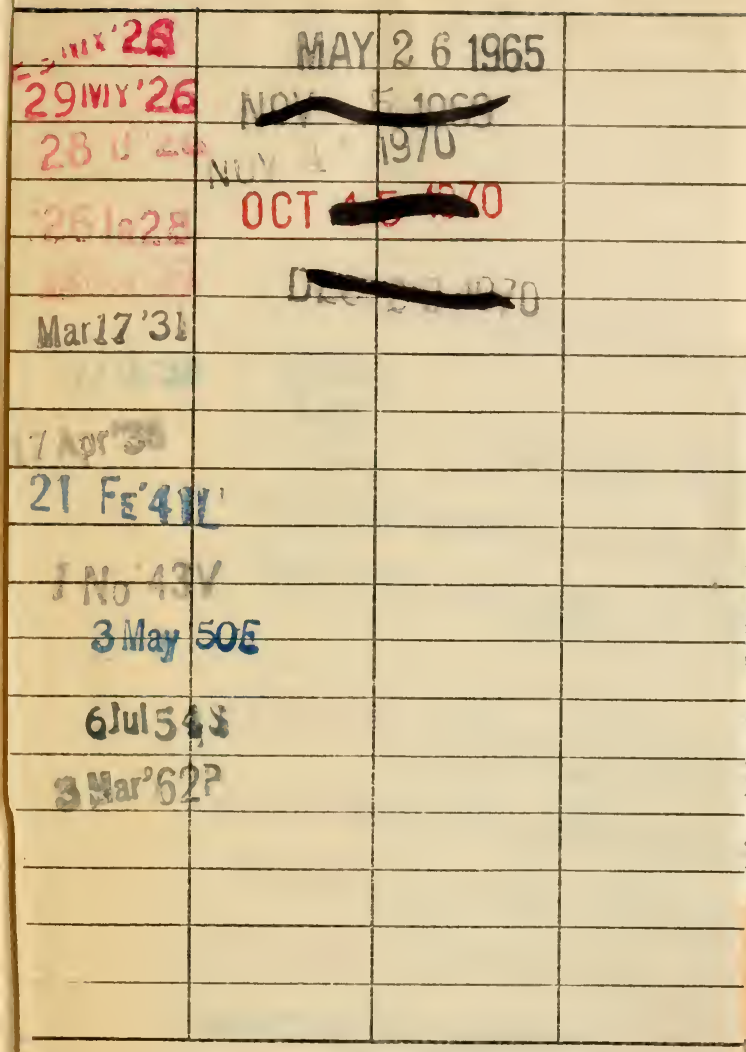

S. and $-403$ IME

L. B. Cat. No. 1137

If lipara

21 FE:ाL है $1 \mathrm{No}^{\circ} 4 \mathrm{By}$

3 May'F

19 May' 


\title{
THE UNIVERSITY OF CHICAGO SCIENCE SERIES
}

\author{
Editorial Committee \\ ELIAKIM HASTINGS MOORE, Chairman \\ JOHN MERLE COULTER \\ PRESTON KYES
}




\section{He UNIVERSITY OF CHICAgo SCIENCE SERIES, established by the Trustees of the University, owesits origin}

to a belief that there should be a medium of publication occupying a position between the technical journals with their short articles and the elaborate treatises which attempt to cover several or all aspects of a wide field. The volumes of the series will differ from the discussions generally appearing in technical journals in that they will present the complete results of an experiment or series of investigations which previously have appeared only in scattered articles, if published at all. On the other hand, they will differ from detailed treatises by confining themselves to specific problems of current interest, and in presenting the subject in as summary a manner and with as little technical detail as is consistent with sound method. They will be written not only for the specialist but for the educated layman. 
PROTOPLASMIC ACTION AND NERVOUS ACTION 


\section{THE UNIVERSITY OF CHICAGO PRESS \\ CHIOAGO, ILLINOIS}

THE BAKER AND TAYLOR COMPANY NEW YORK

THE CAMBRIDGE UNIVERSITY PRESS

LONDON

THE MARUZEN-KABUSHIKI-KAISHA TOKYO, OSAКА, КYОTO, FЕКЕОКА, SENDAI

THE MISSION BOOK COMPANY SHANGHAI 


\title{
PROTOPLASMIC ACTION AND NERVOUS ACTION
}

\author{
By \\ RALPH S. LILLIE \\ Biologist, Nela Research Laboratories, Cleveland; formerly \\ Professor of Biology, Clark University
}

What am I, Life? a thing of watery salt, Held in cohesion by unresting cells .... ?

-Masefield

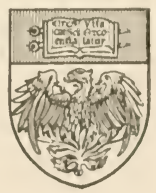

THE UNIVERSITY OF CHICAGO PRESS CHICAGO ILLINOIS 
Copyright I923 By

The University of Chicago

All Rights Reserved

Published October 1923

Composed and Printed By The University of Chicago Press Chicago, Illinois, U.S.A. 


\section{LIBRARY \\ N. C. State College}

TO

HELEN MAKEPEACE LILLIE 



\section{PREFACE}

The present volume is based in part on lectures delivered in Clark University and the Marine Biological Laboratory, on the physico-chemical basis of the more general or fundamental properties of living matter. Common to all forms of living matter are certain properties or modes of action which are absent or imperfectly developed in non-living matter. The chief of these are (I) the property of specific growth, and (2) a unification or integration of activities, of such a kind as to secure the continued existence of the living system in its environment. The question of how the living system must be constituted (in the physico-chemical sense) in order to exhibit such properties is the fundamental one for physiology.

Of late years the analytical investigation of the living organism and its products has made great advances; on the synthetic side, however, progress has been relatively slight. The precise manner in which certain special physico-chemical materials and processes are combined so as to produce life still remains largely obscure. It may be expected that properly directed experiment will throw light on this problem, as it has on many others apparently equally difficult, but at present we are at a stage where exact or scientific knowledge is only in its beginning.

In this book I have made no attempt to consider in detail the many special problems of pure physics and chemistry which are presented by the organism. It is 
assumed that these problems are of the same kind, and to be approached by the same methods, as other problems of physics and chemistry. This point of view seems the only one possible for the scientific investigator. The organism exhibits a regularity which, although of a special kind, is obviously based upon and presupposes the regularity of its component physico-chemical processes. Investigation of the latter requires the use of the exact methods developed by modern analysis; and these have been shown to yield the same constant and reproducible results in organisms as in non-living systems. In fact, one of the most striking features of organic processes is their exactitude, which is frequently safeguarded by regulatory devices of the utmost delicacy. The investigation of many such processes is purely physico-chemical in its method and results.

It must be remembered, however, that in living organisms we are dealing with synthetic products of a higher order. When the materials and energies of the surrounding world unite to constitute the organism, new qualities and modes of activity inevitably come into existence; these special properties of living beings form the subject-matter of the biological sciences, as distinguished from the physical sciences. For this reason the physical and chemical characterization of the constituents, reactions, and processes whose combination or synthesis produces life is not in itself sufficient; the biological interest centers in the conditions and special mode of this combination, and in the nature of the resulting unity. The problem of the nature of vital organization remains the fundamental one for biology. 
We may safely assume that all qualitative phenomena, including those of the living organism, are subject to quantitative laws; but the determination of these laws, while an essential object of scientific investigation, cannot be regarded as its only object. The biologist is primarily interested in the phenomena which are peculiar to life and in the conditions under which these originate and manifest themselves. As already indicated, growth, development, and an integrative correlation of activities are the chief distinguishing characters of organisms. Underlying and determining these properties are the fundamental or universal properties of protoplasm. The essential problem in the physiology of growth (and ultimately of development and heredity) is the problem of the conditions of specific chemical synthesis in protoplasm. And the problem of integration resolves itself largely into the problem of the conditions under which protoplasmic processes, although spatially separated, mutually influence one another; i.e., the problem of transmission. For the solution of these problems we require first of all a knowledge of the special conditions under which the chemical reactions in protoplasm proceed and influence one another.

The general physical conditions under which chemical reactions are initiated, accelerated or retarded, and influence other reactions at a distance are undoubtedly the same in living as in non-living matter; but the special features of composition and arrangement in the protoplasmic system often render detailed analysis difficult. Under these circumstances the study of "models" simple artificial systems in which the action of single factors may be isolated and observed may be of great 
service, and I have made use of this method in a number of instances. For example, the transmission of the effects of stimulation in nerve and other irritable forms of protoplasm resembles closely certain types of chemical transmission or distance-action in metal-electrolyte combinations; many biocatalytic reactions are identical with those induced by colloidal platinum or charcoal; there are also instructive analogies between organic growth and certain types of inorganic growth. Many fundamental physical processes which play an important part in protoplasm are independent of the special chemical composition of the material; thus the influence of radiation and electricity on living matter is a special case of the general influence which these agents exercise under appropriate conditions upon all chemical reactions. The detailed nature of the conditions in protoplasm can be determined only by special investigation.

The more special sections of this book have reference to the two fundamental problems above defined. The structural and physico-chemical organization of living matter, the modifiability of its rate of reaction under varying conditions (irritability), and its transmissive property (so highly developed in nervous tissues) are considered in some detail; and their probable relation to the polyphasic and film-partitioned character of the protoplasmic system is indicated. 


\section{CON'TEN'TS}

CHAPTER

I. Introduction-General Cilaractiristics of I,IN ING MATTER

II. The Cellular Organization of Living Mattir - if

iII. General Characters of Living Organisms

IV. General Peculiarities of Protoplasil as a I'hisik Cal System

V. Physical Nature of Protoplasmic Strlcture: Ise portance of Surface Conditions

VI. Protoplasmic Structure (Contimucd): Perme.bility and Other Properties of P'rotoplasmic MEMBRANES

VII. General Conditions Deterahning the Properties of Protoplasmic Membranes

VIII. Relation of the INorganic Salts of the Medicm to the Physiological Processes in Protoplasm

IX. General Physiological Action of Lipoid-Alter. ant and Surface-Active Substances

X. Catalysis in Relation to the Chemical I'roCESSES IN Living MatTer.

XI. Electrical and Other Factors in the Catalytic Action of Protoplasir

XII. Stimulation and Transmission of Excitation IN PRotoplasM

XIII. Bioelectric Phejomena

XIV. Membrane Changes during Stimulation

XV. The Physico-Chemical Basis of Transmissioy in Nerve and Other Protoplasme Sisticis

INDEX 



\section{CHAPTER I}

\section{INTRODUCTION-GENERAL CHARACTERISTICS}

OF LIVING MATTER

It is a peculiarity of living matter, as distinguished from non-living matter, that it is never found in a diffuse, unorganized, or formless state, but always composing definite individualized systems or organisms, of which there are many kinds or species, each with definite and, on the whole, highly constant physicochemical, structural, and active characters. These organisms form a class of natural systems which, considered quantitatively, is a very small one in comparison with physical nature as a whole. This fact in itself implies that living systems are highly special developments; they represent a higher order of synthesis, and it is to be expected that they should exhibit properties and activities which are absent in non-living systems. Hence the existence of a sharp contrast between the living and the non-living -i.e., between organism and environment-is not in itself surprising. We know, however, that continuous transitions from the one to the other have existed and still exist; life has evolved from non-living matter in the past; and in the present every living organism is the seat of a continual transformation of non-living into living matter. The chief problem of general physiology is to trace the steps of this transition; i.e., to determine the nature of the synthesis by which the living matter, protoplasm, is 
built up from the non-living material which it incorporates from the surroundings.

Physiology regards the living organism solely in its objective aspect as a physical object in external nature; many aspects and manifestations of living beings do not form directly a part of its subject-matter, and the general philosophical question of the essential significance of life in the cosmos - the question of vitalism or anti-vitalism -is not one which it makes any pretensions to answer. It observes simply that certain systems, living organisms, exist in the external world, presenting a remarkable combination of properties not found in other natural systems; and its task is the analysis of these systems in the terms and by the methods of physical science.

These special or distinguishing peculiarities of living organisms may be grouped under several general heads, as follows: (I) metabolism, (2) growth, automatic selfmaintenance, reproduction and heredity, (3) irritability, (4) regulation and adaptation, (5) spontaneous activity, having reference to future as well as present conditions. The essential character and implications of these various properties will first be briefly considered.

\section{METABOLISM}

The essential peculiarity which places organisms in a class apart from most non-living objects is that their properties and manifestations depend on their continued chemical activity; in other words, they are metabolizing systems, formed, maintained, and perpetuated by processes of chemical transformation. The production of new chemical compounds by transformation of other compounds taken from the surroundings, and the 
organization of these compounds in new structural and chemical relationships, constitute the fundamental activities of all living matter. Hence a consideration of the general features of metabolic processes must come first in any discussion of the nature of protoplasmic action.

Under the term metabolism are included primarily the nutritive and energy-yielding chemical processes in protoplasm, and secondarily the other chemical processes subserving or underlying these. The application of the term is usually clear; but metabolic processes comprise chemical reactions of all kinds, many of which are in no sense peculiar to organisms, while others are not met with elsewhere in nature. The traditional distinction between constructive and destructive metabolism remains an essential one; what the organism is at any time is a resultant of the effects of these two large and, in general, oppositely directed groups of chemical reactions. Broadly speaking, the constructive reactions represent the nutritive processes, and the destructive reactions the energy-yielding processes. Constructive metabolism includes the synthetic (anabolic) reactions underlying growth, self-maintenance, and reproduction. In any species the end-products of the constructive sequence of reactions consist largely of certain colloidal compounds, highly individualized and specific in their chemical constitution, the proteins; the other synthetic products (carbohydrates, fats, lipoids, etc.) are chemically nonspecific; i.e., not confined to the species in question; these form, together with the specific compounds, water and various dissolved substances, a complex and highly organized system, or organic individual, which is specific 
(i.e., definitely characterized and unique) in its structural and active characters. This building-up, by means of metabolic construction, of a complex system, specific in chemical composition, structure, and activity, out of relatively simple non-specific materials taken from the surroundings (food, water, salts) is the fundamental general peculiarity which distinguishes living organisms from non-living systems. Constantly associated with the constructive group of reactions is the destructive or catabolic group by which substances contained in the protoplasm are broken down, usually oxidized, to yield the energy freed in vital activity. A great diversity of compounds are thus utilized by protoplasm as sources of energy; the catabolic process is non-specific; i.e., sugars, fats, and proteins are metabolized to yield the same endproducts $\left(\mathrm{CO}_{2}\right.$, water, urea, etc. $)$ and energy in organisms of all kinds.

\section{GROWTH, MAINTENANCE, REPRODUCTION,}

AND HEREDITY

It is essential at the beginning of any study of fundamental vital properties to recognize the dependence of the various phenomena designated by the four terms above upon the fundamental process of specific constructive metabolism. In every organic individual normal self-maintenance, by which the material lost as a result of metabolic destruction is replaced by new construction, involves the same specific synthetic reactions as those concerned in growth. And growth is obviously a highly specific process; this becomes evident whenever a seed or an egg "grows into" the specifically organized adult. Organic growth thus involves or implies "hered- 
ity"; and since growth is the foundational life-process that by which all living matter is brought into existence -we see at once that the specificity of the underlying metabolic syntheses is the essential condition underlying organic specificity. When constructive metabolism ceases, not only does growth cease but life itself, since the continual formation of specific material is a prerequisite for normal maintenance.

Each of the terms above, however, designates a feature or aspect of vital phenomena which is as a rule perfectly definite and distinguishable from the others. Organic growth is perhaps best defined as increase in the quantity of the specifically organized living material. ${ }^{I}$ Reproduction is the formation of new individuals by growth from the parent organism or a detached portion of the latter (germ, gamete); in metazoa the replacement of outworn or senescent individuals is thus accomplished. Reproduction has been defined as "discontinuous growth"; thus the growth of a plant-cutting is a reproduction, and many" cases of asexual reproduction in animals illustrate the same phenomenon (reproduction by fission, regeneration). In the lowest organisms, e.g., bacteria, it becomes no longer a matter of practical interest to distinguish between growth and reproduction. Heredity, the resemblance of offspring or outgrowth to parent stock, is illustrated in all of these cases; the special problem of heredity, therefore, is reducible ultimately to the fundamental problem of the conditions determining the property of specific construction possessed by all foms

${ }^{\mathrm{r}} \mathrm{Cf}$. the discussion by Child, Senescence and Rejurenescence, Chicago (I915), chap. ii. 
of protoplasm. ${ }^{\mathrm{x}}$ This consideration is overlooked in many "theories of heredity," which apparently take for granted the existence of the property which they are called upon to explain. Ids, pangenes, chromosomes, and the other representative particles of these theories are self-multiplying units; i.e., they possess ex hypothesi this automatic power of synthesizing material and structure of their own kind. It is well, therefore, to realize clearly the fundamental identity of the physiological conditions underlying all of the phenomena grouped under the foregoing head.

To prevent any possible misunderstanding, a few words may be added here concerning the nature of the physiological problems raised by the chromosome theory of heredity, which now seems to be established on a secure basis through the correlation of genetic and cytological investigation. ${ }^{2}$

All the evidence indicates that the chromosomes, the carriers of genetic factors or "genes," are the elements or units in a sorting and distributing mechanism, by means of which special formative metabolic processes are localized in definite regions of the growing and

${ }^{x}$ Haldane's remarks in his British Association address of 1908 (Nature, LXXVIII, 555), "nutrition itself is only a constant process of reproduction" and "heredity is for biology an axiom and not a problem," do not dispose of the problem of heredity, but apparently assign it to a border-line position, somewhere between chemistry and biology. The property of automatic specific synthesis is the one to be explained. The original natural systems which exhibited this property were presumably the ones from which living organisms, as we find them, have evolved.

${ }^{2}$ Cf. T. H. Morgan, The Physical Basis of Heredity, Philadelphia (I919); also "The Mechanism of Heredity," Nature, CIX (I922), 24I, 275, 3I 2 . 
developing organism. The property of self-multiplication possessed by these units, on which the possibility of their special action depends, is, however, not peculiar to them, as already pointed out, but is a property of protoplasm and of protoplasmic structures in general. ${ }^{x}$ Once the chromosomes have been produced by this autosynthetic process, they are free to exercise their special influence and function. In this respect they are like other structures which are definite factors in the formative processes; they must first be synthesized by the fundamental growth processes before they can function. There are obvious analogies between the action of the chromosomes and the action of special form-determining chemical substances (or hormones) produced by various organs. Development at certain stages is demonstrably a consequence, as regards certain special features, of the previous development of the thyroid or the pituitary gland or the gonads. An even more general analogy may be pointed out here, since it illustrates the nature of many biological sequences. A prerequisite to the normal activity of the adult is the development of the normal adult structure; for example, the formation of hands must precede the construction of a house, but we do not explain the whole constructive process by reference to the hands, the tools for sorting and distributing the materials. How the chromosomes influence formative metabolism is the essential problem for physiology; this problem is at present unsolved, but there can be no doubt as to the existence of this influence.

${ }^{x} \mathrm{H}$. J. Muller has discussed the properties of the genes from this point of view in a recent paper in American Naturalist, LVI (1922), 32. 


\section{IRRITABILITY}

It is characteristic of all organisms that they respond to changes in their environment (stimuli) by changes in their own activity (response). And since metabolic reactions underlie all vital activity, this fact implies that the chemical reactions constituting metabolism are subject to the influence of external agencies acting upon the protoplasm. Both constructive and destructive metabolism may be thus influenced.

In general, what we mean by irritability is this susceptibility to external influence; irritability, however, cannot be considered as a special property independent of the continual automatic, chemical, and other activity of the living system; its existence merely shows that the chemical reactions of protoplasm are subject to modification-e.g., acceleration or the reverse-under the influence of relatively slight changes of state, caused usually by the action of external agencies upon the protoplasm.

A peculiarity of most intact organisms is that the changes of activity thus induced are normally of such a character as to favor the continued existence of the individual or of the species in the environment; this general fact may be expressed by saying that the normal responses to stimulation, however varied in detail, have a regulative or adaptive character. Adaptiveness, however, is a peculiarity of the organism as a whole, not an inherent property of protoplasm in general; this is shown by the fact that isolated parts may show irritability quite independently of any adaptive reference; e.g., nerve or muscle. In this respect irritability may be compared with the chemical instability of explosives, which may also be applied adaptively. 
4. REGULATION AND ADAPTATION, INTEGRATION

These characters, while based on irritability; have a more distinctively organic or vital quality-are manifestations of a higher plane of organization - than the simple property of responsiveness to stimuli. Fundamentally they are related to the characteristic self-conserving property of the organic individual or species; this property is exhibited by all naturally occurring organisms, i.e., the structure and activity of the latter are of such a kind as to favor a permanent or stable existence in the environment. Under the terms regulation and adaptation, we include, in their broadest application, all of those features of adjustment-structural, chemical, and active-which are especially characteristic of living as distinguished from non-living systems. The organism is "fitted" to its environment; the reciprocal relations between the two are so balanced or correlated that the species persists. In other words, the properties or activities which have special "survival value" are those which we designate as adaptive. Adaptations may be (I) of a static or morphological kind-non-temporal in their reference-e.g., when the structure of the organism shows a correspondence with the unchanging features of its environment. Perhaps the most general and widespread example of such static adaptation is seen in the general plan of bodily structure common to most freeliving animals-bilateral symmetry combined with antero-posterior and dorso-ventral differentiation. ${ }^{x}$ Or

I have discussed more fully the general conditions that render this type of structural plan adaptive in a paper on purposive and adaptive behavior in the Journal of Philosophy, Psychology and Scicntific Methods, XII (I9I5), 589. 
they may be of an active kind; such are classed as regulations. In this case the activity of the organism or of its parts changes in such a way as to resist or compensate departure from the normal; i.e., from the physiological or other conditions required for continued life. The automatic regulation of food-intake, gaseous exchange or temperature, the protective and other self-conserving reactions or instincts, and the phenomena of form-regulation are examples. Since in all such cases the persistence of the organism in the environment is the condition promoted or secured, and since persistence in external nature implies equilibrium, we may characterize regulations as reactions of an equilibrating type; i.e., regulation corresponds essentially to equilibration. In a sense it is obvious that the structure and activities of an organic species must be such as to secure persistence in the environment, since the alternative is extinction; nevertheless the universal presence of regulative modes of activity is a peculiar and highly remarkable feature of living as distinguished from non-living systems, and requires special consideration. Regulations or automatic equilibrations are also met with in many non-living systems (regulators in machines or other artificial systems), but for the most part these are of a relatively simple type.

The conception of organic integration is closely related to that of regulation; the maintenance of a definite and unified structure and activity in any complex system consisting of many parts requires the mutual interaction and control of the different parts in such a manner that the activity of each is subordinated to that of the whole. This integration presupposes the trans- 
mission of chemical and other influence between different regions, and in higher organisms is effected chiefly through the nervous system in co-operation with a chemical control exercised by special substances (hormones and other metabolic products) transported from place to place in the circulation. ${ }^{x}$ The possibility of these two forms of integration rests ultimately on mechanical or structural factors, shown in the permanence of morphological form and organization; hence some authors speak of a mechanical integration (or correlation) in addition to the other two. ${ }^{2}$

\section{SPONTANEOUS ACTIVITY}

The chief vital phenomena classed under this head are characteristic of the organism in its action as a whole, rather than of its special parts, although many of these are spontaneously active; e.g., the heart. They are especially developed in animals, and include spontaneous activity and trains of activity (instincts) directed toward the external world and having usually some definite future reference; purposive and conscious action, in their physiological aspect, also belong here. All such characters are based upon, or presuppose, the other more fundamental characters; i.e., they are not general protoplasmic properties but appear at a higher level of vital synthesis; hence they do not form, strictly speaking, a part of our present subjectmatter.

${ }^{2}$ Cf. Sherrington's Integrative Action of the Nerious Sy'stem.

${ }^{2}$ Cf. Child, The Origin and Development of the Nerrous System from a Physiological Viewpoint, University of Chicago Press (192I), chap. i, p. I2. 


\section{SCOPE OF GENERAL PHYSIOLOGY}

The first four groups of characters appear to be common to all forms of living matter; i.e., they are the expressions of the general or fundamental properties and activities of the living substance or protoplasm wherever found. We class as "living" all natural systems exhibiting these properties in combination; and general physiology has for its object the study of the essential composition and activities of such systems.

From this point of view the distinction between animals and plants becomes one of minor importance. This difference is essentially one of method of nutrition; in plants the processes of constructive metabolism start with more elementary and widely diffused materials than in animals. A brief reference to the main points of distinction seems relevant here, since it may assist in defining the essential problem under consideration.

It is evident that all organisms require for their normal growth and activities the presence of energyyielding (chiefly oxidizable) materials in the protoplasm, as well as materials for building up protoplasmic structure; the chief representatives of these two classes of substances are, respectively, the carbohydrates and the amino-acids. The main differences between plants and animals relate to the methods by which these materials are obtained or rendered available. In green plants they are synthesized from simpler compounds which in their unaltered state cannot serve as sources of energy$\mathrm{CO}_{2}$, salts, water. In animals the chief "food" materials are already complex compounds of high chemical potential which are not synthesized in the organism but are prepared outside of the latter (ultimately by 
plants), and are introduced into the organism from without by its own special activities. In both groups, however, the active living substance or protoplasm consists chiefly of compounds of the same general chemical type, which in both cases undergo similar transformations. The fundamental physiological processes of plant and animal cells are thus closcly similar. Hence, in general physiology, whose aim is the analysis of the vital process, wherever occurring, organisms of both groups come equally under consideration. 


\section{CHAPTER II}

\section{THE CELLULAR ORGANIZATION OF LIVING MATTER}

General physiology has been defined by Verworn ${ }^{\mathrm{x}}$ as "cellular physiology," in accordance with the general conception of the cell theory that the ultimate living units of any organism are the cells. According to this conception the cells are the simplest units capable of independent life; hence general physiology, aiming at the analysis and characterization of life-processes, should be equivalent to cell physiology. There appears, however, to be a certain arbitrariness in this idea. The cell is already a complex system with a definite organization, usually containing a nucleus and exhibiting other special structural differentiations. The question of the physiological significance of the cellular organization constitutes a special problem in itself. While it is remarkable that all higher organisms show this type of organization, it seems hardly justifiable to regard all organisms as consisting of cells and products of cells. Such a conception regards the simplest living unit as having a certain definite type of structural organization; i.e., it is essentially a morphological conception. A chemical characterization seems to meet the requirements of the case more completely. Many organisms are known which do not show the chief structural feature of the cell, differentiation into nucleus and cytoplasm; e.g., bacteria and blue-green algae. Usually bacteria are regarded as plant cells of a special kind; it is questions Allgemeine Physiologie, 5 th edition, Jena (I 909 ), chap. i. 
able, however, if micrococci, and especially the organisms in filterable viruses, can be considered as cells in the true sense. The case of the ultra-microscopic organisms present in the filterable viruses is of special interest. These organisms can be demonstrated only by the effects which they produce (infection); they prove themselves to be living by their power of automatic growth, shown by multiplication in the body of the host or in culturemedia, and also by exhibiting other properties characteristic of protoplasm in general, such as thermolability and susceptibility to toxic agents of the disinfectant class. They may be described as complex and chemically: active (metabolizing) material in a fine state of subdivision (like that of colloidal material), possessing in addition to the other properties of matter in this state the special vital properties of assimilation, growth, and multiplication. As already pointed out, this ability to transform environmental material into its own specifically organized and active substance is the distinctive criterion of living as distinguished from non-living matter.

Our conceptions of the nature of living organisms must be broad enough to include the ultra-microscopic forms. Cells, as found in higher organisms, are units of a relatively complex and highly differentiated kind, representing a comparatively advanced stage of evolution. They are by no means to be regarded as the only: systems in nature exhibiting the characteristics of life.

\section{THE CELL}

In higher organisms, however, we have definite experimental evidence that the smallest unit capable of continued independent life is the nucleated cell. The 
protozoa remain as single cells throughout life. The higher animals and plants are single cells only at the beginning of their development-in the germ-cell stage; in later developmental stages and as adults they consist of large, closely associated aggregates or colonies of cells, which, together with the intercellular fluid media serving for transport (blood) and various other products of cellular activity (skeletal and other structures), form a complex and highly integrated system, or organic individual. Each cell in this organism is to be regarded as living and capable of independent existence under appropriate conditions.

The statement that single isolated cells are capable of independent life has been shown experimentally to be true, not merely of organisms which throughout their life are unicellular, but also of many of the cells of higher organisms when isolated under favorable conditionsleucocytes, ciliated cells, muscle cells, tissue-cells. Epithelial cells will grow in suitable culture-media; embryonic nerve cells, isolated in sterile plasma, send out axones in a characteristic manner; i.e., retain the normal power of growth, differentiation, and development; ${ }^{2}$ and many functional adult cells continue to live and grow when isolated under favorable conditions of food and oxygen supply. ${ }^{3}$

On the other hand, experiment shows that for normal and long-continued vital activity the cell must be

${ }^{x}$ Cf. L. Loeb, Arch. Entwickl. Organ., XIII (I902), 487, and earlier papers there cited.

${ }^{2}$ Harrison, Proc. Soc. Exp. Biol. and Med., III (1907), 140; Journal of Experimental Zoölogy, IX (1910), 797; XVII (19I4), 521.

${ }^{3}$ Cf. Carrel and Burrows, Journal of Experimental Medicine, XIII (I9II); W. H. and M. R. Lewis, Anatomical Record, VI (IgI 2). 
complete, at least in the sense that both nucleus and cytoplasm (or portions of both) are present. This is shown by experiments on enucleated cells, such as egrg cells; portions containing nuclei survive; the others die. But if enucleated portions are fertilized and thus furnished with nuclei, they continue to live. ${ }^{x}$ An otherwise complete protozoön such as Stentor will die if deprived of its nucleus, while fragments of less than one-twentieth the normal size will survive and reform a complete organism if a portion of nucleus is present. ${ }^{2}$ Similar results have been obtained in experiments on other protozoa; e.g., Verworn's with Thalassicolla. ${ }^{3}$

There is a large body of similar experimental fact indicating that the continued interaction of nuclear and cytoplasmic components is an essential feature of normal cell-metabolism. It is usually supposed that the nucleus has special relations to synthetic metabolism; hence its special importance in growth and regencration, but the whole problem of the relation of nucleus to cytoplasm is at present in an unsatisfactory state. ${ }^{4}$

It is clear, nevertheless, that the nucleated cell of the higher organism is a complete and autonomous living unit. But in view of what has just been pointed out regarding the non-cellular or subcellular constitution of some organisms, we must avoid regarding the distinctively cellular features of protoplasmic organization

${ }^{x}$ Cf. Delage, Arch. de zool. exp. et gén., VII (1899), $38_{3}$.

${ }^{2}$ F. R. Lillie, Journal of Morphology, XII (1896), 239.

3 Verworn, Arck. ges. Physiol., LI (ISgr); cf. also Allgcmeine Physiologie, 5th edition (I909), p. 620.

4 For a recent study cf. V. Lynch, American Journal of Physiology, XLVIII (1919), 258. 
as the all-essential ones. To do so would be to imply that in the early or precellular stages of organic evolution the assimilative or proliferative types of colloidal material, which presumably were then the only systems representing organisms, were not living. The formation of a particular kind of structure is not the essential criterion of vitality; the properties which underlie the formative or structure-building activities are the primary ones. In most animals and plants these activities give rise to a cellular type of structure, but this is not necessarily true of all.

\section{IMPORTANCE OF CELLULAR ORGANIZATION}

There is a sense, therefore, in which we may regard the cellular type of structural organization as not so much the cause or necessary condition of the vital activities as their product or effect. Obviously all cellular organisms come into existence through the constructive processes of growth. This was pointed out by Huxley, in the early years of the cell theory, in a well-known passage in which he speaks of the cells as being not the producers but simply the products or indicators of vital action. Like the shells on the sea beach the cells "mark only where the vital tides have been and how they have acted."' This comparison is an apt one in that it emphasizes the primary importance of the structure-forming vital activity which expresses itself in the formation of cells; but it tends perhaps to subordinate the part played by the cellular structure, once it has been attained. There is no doubt that this

"Cf. Huxley, "Review of the Cell Theory" in the British and Foreign Medico-chirurgical Revierw (1853). 
structure determines, in a quite special way, the character of the protoplasmic processes; i.e., has its own definite causative and controlling influence. The metabolic and other cell processes can be shown to be profoundly. influenced by changes in the physical and other state of cell structures. For example, there is evidence that in most cells irritability depends primarily upon the special properties of the external protoplasmic layer or plasma membrane; the contractile, secretory, and similar mechanisms are cell structures; the special relation of the nucleus to constructive metabolism has already been mentioned. In general we may say that physiological activity in all higher organisms is intimately bound up with the special features of structure, chemical organization, and activity peculiar to cells.

A universal peculiarity of living matter, considered simply as a chemical reaction-system, is that its principal chemical reactions, especially the specific constructive group, occur under the control of structural conditions. If protoplasmic structure is destroyed, mechanically or otherwise, these essential vital reactions at once cease. New structure as it arises in growth or development must therefore have a modifying influence on the metabolic processes and the other physiological processes dependent upon these. The structural characters peculiar to cells cannot fail to influence profoundly the chemical activity of all living systems having the cellular type of organization. One of the fundamental problems of general physiology has reference to the special nature of the relations existing between cellular structure and the chemical processes of the cell protoplasm. 
The question, Why is living matter so characteristically cellular in structure, seems to be equivalent to the question, Why is it partitioned, subdivided into minute, usually microscopical, portions (cells), or structurally discontinuous? Each portion of protoplasm is separated from its surrounding medium or from adjoining cells by a thin, structurally distinct boundary layer usually called the "plasma membrane"; and the presumption is that some definite physiological advantage attaches to this peculiarity. The most evident general answer is that this layer serves to separate or insulate the living protoplasm from the surroundings, and thus to protect it from the disintegrative or otherwise adverse influence of the latter. This view regards the plasma membrane as primarily a protective structure. Through its presence each separate portion of living substance, or cell, is enabled to retain its special composition and individuality. But this answer, while undoubtedly correct in part, is too vague and general to be satisfactory. The recent experimental studies on protoplasmic permeability have thrown a more definite light on the problem. They have shown that in typical living cells the external protoplasmic layer has the properties of a semi-permeable membrane; i.e., it is impermeable or difficultly permeable to the water-soluble substances of low molecular weight present in the protoplasm and surroundings (and to chemically similar substances), while freely permeable to water. Free diffusion of soluble substances either into or out of the cell is thus prevented; the protoplasm can preserve a chemical composition different from that of the surrounding medium without the interference that would result 
from unrestricted diffusive interchange. It is evident that if a minute portion of protoplasm is to retain its special chemical organization, it must be protected against loss of its water-soluble constituents by diflusion, and also against the unregulited entrance of soluble. substances from without. Chemical analysis shows in fact that the crystalloidal content of living cells is typically widely different from that of the surrounding medium. $^{2}$ 'The presence of a diffusion-proof partition separating each small portion of living protoplasm from its surroundings is apparently an essential feature of the cellular organization.

Without such a diffusion-hindering type of structure. it is difficult to see how a high degree of chemical difierentiation could be maintained in such a system as the living organism, consisting, as it does, in large part of an aqueous solution of diffusible substances. Difierences in the distribution of soluble substances between protoplasm and surroundings would tend to equalize themselves by diffusion, and chemical differentiation would become difficult or impossible. Morphological difierentiation has long been recognized as favored by the subdivision of the developing germ into cells: this condition permits morphogenetic processes in neighboring cells and cell groups to proceed in relative independence of one another. ${ }^{3}$ In a similar manner an essential

${ }^{x}$ Cf. my paper in Biological Bulletin, XVII (so0g), isS, for a fuller discussion.

2 For a summary of work in this field, if. Hober's Phyentabiate Chemie der Zelle und der Geucbe (1914), pp. 370, 40.; (f. alwo Bottenein article in Winterstein's IIandbuch der vergl. Physubl, I (10) 11), 37.

${ }^{3}$ Cf. F. R. Lillie, "Adaptation in Cleavage," Heods Hele Bidlunnod Lectures (1899), p. 43. 
condition for the isolation of chemical and physiological processes in adjacent regions of the organism is the presence of the semi-permeable intercellular partitions.

There is also evidence that the internal protoplasm of the single cell is frequently pervaded by a system of films or closed partitions giving a chambered type of structure; and the possibility of intracellular chemical differentiation ("chemical organization") has been referred to this condition. ${ }^{I} \quad$ Such a chambered structure corresponds essentially to that of an emulsion-like or alveolar system. Apparently any physico-chemical system which is built up largely of water and substances in aqueous solution must be a partitioned system if it is to maintain within a small space a high degree of chemical differentiation together with a corresponding diversity of chemical activity.

In general, each living cell can be shown to possess a surface layer (plasma membrane) with properties different from those of the internal protoplasm. At the boundary between this surface layer and the adjoining medium the general phenomena characteristic of phase-boundaries are exhibited. A highly characteristic feature of the living cell is that its surface is sharply defined against the medium, like the surface of an oil drop, very much as if the surface layer consisted of water-insoluble material. This water-immiscible property of living protoplasm and the semi-permeability of its boundary layer are closely associated properties; together they constitute one of the most noteworthy physical peculiarities of living protoplasm. Especially significant is the

${ }^{x}$ Hofmeister, Die chemische Organisation der Zelle, Braunschweig (IgOI). 
fact that they are preserved only while the cell remains living. All cells disintegrate on death; the vital semipermeability and water-immiscibility are then lust. Any living cell, such as a blood corpuscle, suspended in its normal medium, exhibits general physical properties similar to those of a suspended insoluble particle; e.g., an oil drop. These properties are largely an expression of general physical conditions present at all boundary surfaces between adjacent phases, and their consideration becomes of great importance to the physiologist.

In common with other boundary surfaces between mutually immiscible phases the coll surfaces have characteristic electrical properties (interfacial potential differences), exhibit surface tension, and possess the property of condensing or absorbing dissolved substances from the surrounding solution (adsorption). 'The general rôle of adsorption in protoplasmic activity is a highly important one, to be considered later in more detail; and undoubtedly this process is a chief factor in the catalytic or quasi-catalytic action of living matter. In general, the catalytic properties of finely divided substances, such as charcoal and colloidal metals, are referable - at least in large part - to adsorption, and the same is probably true of the cataly tic properties of living cells. Adsorption appears also to be a factor in the collection of nutrient and other substances from very dilute solution, also a highly characteristic feature of protoplasmic activity.

These considerations show that in addition to limiting diffusion and thus providing for structural and chemical differentiation in the manner indicated, the cellular or partitioned structure of living matter is physiologically. 
24 PRकTOPLASMIC ACTION AND NERVOUS ACTION importan't because it furnishes the conditions for another kighy characteristic group of properties, those dependent Honfurface conditions. The protoplasm is thus enabled to utilize (so to speak) the special physical properties exhibited by matter at boundary surfaces. With fine subdivision the proportion of surface protoplasm to the total mass of living substance is large, and the rôle of surface processes assumes corresponding importance. This general point of view recalls Herbert Spencer's explanation of cell-division as essentially a regulative process, the effect of which is to maintain a certain minimal surface-volume ratio in the protoplasmic mass. The living substance enters into relation with its surroundings through the intermediary of a surface layer, which has special physiological properties, in correspondence with the special nature of the physical conditions resident at boundary surfaces. Evidence will be presented later indicating that these electrical, adsorptive, and catalytic properties of the protoplasmic surface layers determine many of the most characteristic features of protoplasmic activity, especially the automatic and rhythmical processes, the susceptibility to electrical influence, and the various manifestations of irritability. 


\section{CHAPTER III}

\section{GENERAL CHARACTERS OF LIVING ORGANISMS}

\section{CHARACTERS OF ORGANISMS IN RELATION}

TO ENVIRONMENT

All organisms have the power of self-maintenance; i.e., of maintaining their identity and a certain constancy of structure, chemicąl composition, and activity in spite of continual changes in their surroundings and in their own living substance. The degree of environmental change to which different organisms are exposed varies greatly, and many cells of higher animals pass their whole life in media which are automatically secured against all but slight variation. On the other hand, protoplasmic activity, implying chemical change, is uninterrupted during life; and, as already pointed out, is largely the expression of chemical reactions, chictly oxidative in nature, by which energy is freed. In all organisms part of the energy thus freed takes such a form that the organism is enabled to maintain itself in equilibrium with its surroundings, grow, and eventually reproduce itself. A curious and highly characteristic cycle of activity is thus shown; thus the animal uses its muscular energy, derived from the oxidation of carbohydrate, to secure more carbohydrate and other materials which serve as sources of vital energy; and this cycle, regulated in accordance with the varying physiological requirements, is repeated continually. throughout life. Such facts illustrate the general dependence of life upon the interchange of material and energy: 
with the environment and explain why so large a part of biological investigation has reference to the interrelations between organism and environment.

We may here recall Spencer's characterization of life as essentially a continual adjustment of internal to external relations. ${ }^{\mathrm{I}}$ Such an abstract definition, however, applies to many other systems found in nature; e.g., to any system in "dynamic equilibrium," such as a candle flame, a whirlpool, or other physical system in which there is an automatically regulated balance between the material and energy supplied to the system and that lost to the environment. Nevertheless, it is peculiarly true of organisms that their processes are of such a kind as to maintain constantly a certain special complex of structural and active characters in spite of internal and external changes. The requirements for such maintenance vary in the different cases, but certain conditions are universal. The primary condition is that material must be taken from the outside that will serve (I) as a source of energy (to replace substances consumed in supplying this energy) and (2) as building material for the structural substratum (protoplasm) in which the energy-yielding transformations occur; in this second class are included substances which do not serve directly as sources of energy-e. g., inorganic salts. Considered from the most general point of view, therefore, the living organism exhibits (I) a continual transformation of material taken from its surroundings into its own specifically organized substance; and (2) a continual chemical decomposition of portions of this substance of such a kind as to furnish free energy which is utilized

× Principles of Biology. 
by the organism in the characteristic activities (foodseeking, etc.) required for its individual maintenance and the perpetuation of its kind.

From this general point of view the simplest cases are the most instructive; e.g., that of a single yeast cell or bacterium introduced into a nutrient medium. The organism grows and divides until eventually in place of the single cell there are thousands. Evidently the material of these additional cells comes from the surrounding medium, certain constituents of which are transformed into the living material or protoplasm. The total quantity of material in the whole system, organism plus culture-medium, is unaltered; but its condition has undergone a profound change. A typical nutrient solution for yeast (Pasteur's solution) contains sugar and various salts ( $\mathrm{NaK}$ tartrate, chlorides, phosphates, and sulphates of $\mathrm{Na}$ and $\mathrm{K}$ ) together with water and oxygen. From these relatively simple materials are built up proteins, lipoids, fats, and other complex bodies; not only are these characteristic substances synthesized but they are distributed or arranged (partly in solid form) in a definite and constant manner so as to give rise to numerous complex and uniformly constituted systems, the yeast cells. Each of these, once formed, becomes the seat of further transformations of the same kind; and by a repetition of this process the non-living material of the medium is progressively transformed into living protoplasm. The transformation is constant and specific, chemically, structurally, and physiologically; "heredity" receives here its simplest manifestation."

${ }^{x}$ Cf. my paper, "Heredity from a Physico-Chemical P'oint of V'iew, Biological Bulletin, XXXIV (1918), 65. 
All organisms and all cells without exception possess this power, that of transforming certain materials selectively appropriated from the surroundings into their own specifically organized and chemically active living substance. The materials used by different organisms vary widely in chemical character and accessibility-contrast the case of a yeast cell growing in a culture-medium with man in his complex social environment-but in every case the essential process is the transformation of non-living environmental material into living substance of a constant and characteristic organization and activity.

The general as well as the special features of the organization of any living being are an index of the nature and accessibility of the environmental materials required for its maintenance. This is well illustrated by the general morphological and physiological contrast between animals and plants. Since in plants constructive metabolism begins with simple mobile or diffusible materials $\left(\mathrm{CO}_{2}\right.$, water, salts), present everywhere in the soil and atmosphere, there is no need for locomotion; and these organisms lead typically a stationary existence, remaining rooted to one spot where the necessary materials can reach them by diffusion. The typical radiating, branching, or dichotomous habit of growth, reaching out into all directions of space and thus providing a large area of surface for interchange, is an "adaptation" to this general environmental condition. Sessile animals also tend to acquire a radiating plan of structure, as illustrated in coelenterates and echinoderms. In the great majority of animals, however, the food supplies have to be selected from an environment containing 
relatively little utilizable and much non-utilizable material. In such a case self-maintenance demands, in addition to the ability to move from place to place, a selective power of reaction by which food materials may be picked out and incorporated. Accordingly the responsiveness to external changes (irritability, motor activity) reaches its highest development in this group of organisms. The development of locomotor powers is especially characteristic of animals; related to this is their great variety of reactions and instincts. From such general considerations we may see in a general way how the distinguishing or prevailing characters of each group have arisen in evolution in correspondence with the differences in their methods of nutrition.

In all organisms this selection of assimilable material from the environment and its transformation into living protoplasm proceed automatically and are regulated in correspondence with the physiological requirements, as these vary with the changes of activity and of external conditions. Both the automaticity and the regulated character of these activities are well illustrated by the changes in the reaction of animals to food materials during periods of "hunger." Consumption of the energy-yielding reserves within the living protoplasm leads to an increased reactivity of the whole organism to these substances. Through this means the maintenance of the metabolic equilibrium is assured under the usual conditions. Regulation of this kind is shown to a greater or less degree by all organisms, and constitutes a fundamental condition of self-preservation; typically if the organism is deprived of any substance or condition necessary for maintenance, its reactivity and behavior 
are altered in a manner tending to compensate or remove the deficiency. Thus hunger is, physiologically speaking, increased reactivity to food materials; thirst is increased reactivity to water; the respiratory center of vertebrates increases its rhythm as $\mathrm{CO}_{2}$ accumulates in the blood; when the oxygen in the water is decreased, the gill-cilia of the fresh-water clam beat more vigorously. ${ }^{x}$ These and many other instances illustrate the manner in which a physiological deficiency may itself furnish the means of setting in motion some physiological mechanism which remedies the deficiency. ${ }^{2}$ The end-effect of all such regulatory responses is to further the persistence of the organism in its environment. As already mentioned, the term adaptive is usually applied to those special peculiarities of structure and activity by which the organism is automatically conserved in spite of environmental change; hence, from the present generalized point of view any active adaptation may be regarded as a special kind of regulation. It is evident that all such regulations are based upon a highly developed irritability; this fundamental property of irritability, therefore, controls all of the active relations between organism and environment, including the interchange of material and energy which is the essential feature of such relations.

\section{SPECIFIC CHARACTERS OF ORGANISMS}

The constructive metabolic processes which build up the living system involve the synthesis of a multiplicity of new chemical compounds from the food materials and

${ }^{\mathrm{I}}$ Cf. Babak, Z. allg. Physiol., XV (1913), I84.

${ }^{2}$ In Pflüger's aphorism, in living organisms "the cause of the need is the cause of the satisfaction of the need." 
other substances (oxygen, salts, water) furnished by the environment. Of these synthesized compounds the most individualized and specific are the proteins. These compounds, characteristically colloidal in their physical properties, constitute, together with certain other materials, chiefly lipoid, the relatively stable, solid, or permanent (structural) portion of the protoplasmic complex.

It is significant that the chief structure-forming compounds should be at the same time those which are chemically the most specific. Specific form and structure are the most obvious peculiarities of the living organism; hence species are usually distinguished by their structural characters. It is to be remembered, however, that the chemical and physiological characters are equally constant and definite, and must be included in the complete characterization of any species. The essential fact, requiring physiological explanation, is that each individual animal or plant resembles, structurally. chemically, and physiologically other individuals of the same species, while differing from those of other species. As already indicated, the physiological basis of this specificity is to be sought in the specific nature of the chemical processes by which the organism is synthesized. We find in fact that a chemical specificity, corresponding to the specificity of the organism as a whole, is exhibited by its constituent proteins, and apparently by these compounds alone. The other chief biochemical compounds (carbohydrates, lipins) are chemically identical in widely differing species, while the proteins vary in their detailed chemical character from species to species. Apparently each native protein has 'a special composition 
and stereo-chemical configuration, by which it is distinguished from the corresponding proteins of even nearly related species. This general fact of an association between specific chemical composition and specific organic structure indicates, together with other evidence, that the specific chemical characters of the structural proteins of any organism determine, in a manner which cannot be defined in detail at present, its specific peculiarities as an organic species. ${ }^{\mathrm{I}}$ Apparently this chemical specificity determines the more intimate protoplasmic structure, and hence indirectly the protoplasmic activities, chemical and other, which are the correlative of that structure and determine ultimately the physiological and other peculiarities of the species.

The problem of the conditions of specific formdetermination in organisms has its special physiological aspects; but on the purely physical side its closest affiliations are with the problem of the relations between the chemical constitution of compounds and their crystalline or other molar structure. When similar molecules unite to form larger molar aggregates, definite regularities of form and structure usually make their appearance; this is especially true when substances separate from solution to form crystals; the axes and angles of the crystal form are an index of the orientation which the molecules assume as the aggregate is built up, and of the linear proportions of the molecules. In most solid compounds this association of structural specificity with chemical specificity can be readily

${ }^{x}$ Cf. Loeb's recent discussion in his Organism as a Whole from a Physico-Chemical Viewpoint, New York (I9I6), chap. iii, "The Chemical Basis of Genus and Species." 
demonstrated; i.e., each compound has a definite and characteristic crystalline form, which is similar for compounds of similar chemical configuration (law of isomorphism). In colloidlal compounds like proteins, crystals are less easily produced, but under appropriate conditions many of these compounds can be crystallized, and it is then found that corresponding or homologous proteins from different species form crystalline aggregrates which differ characteristically in their specific formcharacters. Specificity of crystalline form has been demonstrated most clearly in the case of the hæmoglobins; i.e., the hæmoglobin crystals of the domestic cat differ in a definite and constant manner from those of other species of the same family, and in different vertebrates a general correlation between similarity of crystal form and nearness of relationship can be recognized. ${ }^{2}$ Such facts indicate that as the molecules unite in the process of crystallization to form larger aggregates. structures are built up having definite morphological characters which are determined by the special configuration of the hæmoglobin molecule. 'The growing crystal mass takes on definite form characters, like the growing germ. We may assume that in the living cell, as it grows and differentiates, similar conditions determine the physical state assumed by those proteins which are laid down as microscopic aggregates or deposits to form the

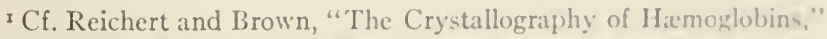
Carnegie Institution Publication No. II6, Washington (1900); also Reichert's paper, "The Germ Plasm as a Stcreochemic System," Science. XL (I9I4), 649. Nuttall's work with precipitin reactions demonstrates a similar correlation between the chemical specificity of proteins and blood relationship. (Nuttall, Blood Immnnily and Blood Relationships. Cambridge University Press.,[1904].) 
protoplasmic structures; in such a case a specific protoplasmic and ultimately a specific cellular structure would be produced, corresponding to the specific constitution of the structure-forming compounds.

The general nature of the relation between stereochemical configuration and crystalline form is best illustrated by Pasteur's classical investigations on the tartrates. The characteristic spatial arrangement of the atoms in the $\mathrm{d}$-tartrate molecule is evidently what determines the production of the specifically formed asymmetric crystals of this compound. Similarly constituted molecules have a tendency to segregate, hence the dextro- and lævo-groups in the solution of the racemic salt unite separately to form separate crystals. The importance of such conditions in the chemical processes of protoplasm is illustrated in the characteristic relations existing between the stereo-configuration of asymmetric compounds and their assimilability, fermentability and physiological action. The possibilities of a specificity based on stereo-chemical configuration are at a maximum in compounds like proteins, built up of chains of asymmetric amino-acids. As we have seen, chemical specificity implies structural specificity in the aggregate formed from such molecules.

As is well known, the chief proofs of the chemical specificity of closely related proteins are derived from immunological and related phenomena. Antigenic properties are apparently confined to proteins, and this peculiarity is of fundamental importance in relation to the whole problem of the conditions of specific synthesis in organisms. When a foreign protein is introduced into the tissue-media of higher animals, one of its physiological 
effects is to alter constructive metabolism in the cells of the organism in a definite manner so as to give rise to other compounds (apparently also protein) of related or complimentary configuration. These new compounds, anti-bodies, form specific chemical unions with the antigens, and hence may serve as a means of identifying the latter or of distinguishing between nearly related proteins, as in the precipitin and anaphylaxis reactions. The living protoplasm responds to the presence of the antigen by synthesizing a compound of similar or complementary configuration; and this chemical resemblance is what determines the intimacy and specificity of union in the antigen-anti-body reaction. The anaphylactic guinea-pig is in fact the most sensitive means at our disposal for distinguishing between proteins of nearly related composition. ${ }^{x}$

It is evident that such phenomena have a most important bearing on the question of the basis of organic specificity. They indicate not only that the synthesis of specific compounds by living protoplasm is determined by the presence of other specific compounds, a fact of general application in the theory of growth processes, but also that the specific syntheses characteristic of a species may be modified under the influence of compounds having a different configuration from those normally present. The indications from precipitin and other tests are that the chemical resemblance between the corresponding proteins of different species is greatest when the biological relationship is closest-when the species are structurally and physiologically most closely similar and in general decreases as the organic difference increases.

${ }^{r}$ Cf. S. Flexner, Science, LII (1920), 6r 5. 
The general conclusion seems therefore justified that the specific biological characters of an animal or plant depend ultimately upon the specific chemical characters of its proteins. The developing germ, or the growing and metabolizing organism, builds up proteins of specific constitution, and these, since they determine the specific structural characters - with the correlative physiological activities - of the organism, form the basis of its biological specificity or special singularity as an organic species. A fundamental problem, therefore, relates to the condition determining the synthesis of proteins of its own specific type by each form of protoplasm. This problem is as yet unsolved. Apparently the presence of proteins of a certain composition and configuration promotes or "catalyzes" the formation of proteins of similar or complementary configuration. A general condition comparable with autocatalysis ${ }^{\mathrm{x}}$ thus determines the specific character of the protoplasmic syntheses, but such a statement merely defines the problem without solving it. The problem, however, cannot be solved before it is clearly defined, and its solution would unquestionably represent a great advance in biological knowledge, since it would involve the solution of the fundamental problems of growth and heredity.

There is some evidence of an identity, or at least close chemical resemblance, between the specific proteins of adult tissues or organs and corresponding or repre-

${ }^{3}$ For the comparison of organic growth with autocatalysis cf. J Loeb, Biochem. Zeitschrift, II (I906), 4I; T. B. Robertson, Arch. Entwicklungsmech., XXIV (I908), 58I; Wfg. Ostwald, Roux's Vorträge und A ufsätze, V (1908). Chodat made a similar comparison for plant growth in 1905 (cf. D'Arcy Thompson's Growth and Form, Cambridge University Press [I9I 7], p. I32). 
sentative proteins in the germ cells. Guyer ${ }^{\mathrm{I}}$ has recently found that the germ cells of rabbits which have been injected with anti-lens serum (formed by immunization in fowls injected with crushed rabbit lenses) are so modified as to give rise in development to rabbits having defective lenses and otherwise abnormal eyes. These defects are transmitted hereditarily by either ova or spermatozoa through several generations. Since anti-bodies attach themselves to proteins of corresponding configuration, these observations are evidence of the presence of the specific lens proteins (or proteins closely corresponding) in the germ cells. Results of an analogous kind recently reported by Detlefsen and Griffith may possibly have a similar significance; rats which had been subjected to prolonged rotation gave rise to offspring showing characteristic defects in equilibrium and tendency to circus-morements, and those abnormalities were also heritable. ${ }^{2}$

While it is difficult to believe that all, or even more than a very few, of the proteins in the adult body are represented by corresponding proteins in the germ, yet it seems not improbable that there may exist some correspondence of a general kind between the chemical organizations of adult and germ, analogous to or paralleling the general morphological correspondence which Conklin's work ${ }^{3}$ has demonstrated between the eggss

${ }^{x}$ Guyer and Smith, Journal of Experimental Zoülogy, XXVI (I gih), 65, and XXXI (1920), 17r. Cf. also American Vaturalist, LV (1921), 97, and LVI (1922), 80.

${ }^{2}$ Cf. Griffith, Science, LVI (1922), 676 .

${ }^{3} \mathrm{Cf}$. Conklin, IIcredity and Environment in the Decelopment of Man, Princeton University Press (1918); also his paper, "The Share of Egg and Sperm in Heredity," Proceedings of the National Academy of Science, III (1917), Ior. 
and the larval stages in certain animals. That is, certain proteins with a basic or fundamental relation to the organization of the species may be chemically identical in adult and germ; and they may even be distributed spatially in a similar way in both; e.g., with reference to the main axes. In this sense a chemical continuity between germ and adult may exist, corresponding to the morphological continuity. At present, however, we are completely ignorant regarding the details of this correspondence and can only await the results of further investigation.

\section{EXPERIMENTAL MODIFICATIONS OF GROWTH}

\section{AND HEREDITY}

If the metabolic production of proteins of specific configuration constitutes the essential chemical basis of growth and development, it must also form the basis of heredity, since by "heredity" is meant not a separate phenomenon but simply the similarity of the constructive or developmental process in the successive generations of a particular organic species. We may therefore regard the factors of growth as identical with the factors of heredity, and apply the same type of physiological analysis in both cases.

We find experimentally that while under normal conditions development follows a highly definite and constant course in each species, it can be altered in a definite manner by various procedures; and a large part of experimental embryology is concerned with modifying the growth processes in the germ or embryo and thus controlling the rate and character of development. In this manner it has been shown that constancy 
of development in any particular species requires constancy in the external conditions. For example, the developing sea-urchin larva forms a skeleton of a characteristic and often complex design in sea water and in artificially balanced media containing the chief salts of sea water together with some sodium carbonate; the formation of this skeleton causes the larva to assume the triangular and long-armed shape characteristic of the pluteus stage. But if the carbonate is omitted from the medium, the skeleton fails to form, and development does not proceed beyond the gastrula stage. ${ }^{\mathrm{I}}$ The special form of the skeleton is said to be "inherited"; this experiment shows, however, that it is dependent on the presence of carbonate quite as much as on the presence of special determinants in the germ.

Such an example shows further that constancy in the normal sequence of growth processes is the essential condition for the manifestation of heredity; it also illustrates the composite nature of the physiological factors determining the production of any adult formcharacter; in all cases the co-operation of definite "internal" and "external" factors is necessary to yield the final result. Many cases are also known where development is altered in a definite manner by the addition of special growth-modifying substances; a wellknown example of such influence, exerted by a simple inorganic substance, is the production of cyclopia in fishes by increasing the magnesium content of sea water ${ }^{2}$ other substances and conditions (alcohol

I J. Loeb, American Journal of Physiology, III (1900), 44r.

2Stockard, Arcli. Entwickl. Organ., XXIII (1907), 249; Journal of Experimental Zoölogy, VI (Igo9), 285. 
anæsthetics, cyanide, cold) have a similar effect. ${ }^{\mathrm{x}}$ These substances hinder or suppress the growth of the anterior region of the forebrain between the optic vesicles so that the latter tend to approximate and coalesce, producing a single instead of a double structure. ${ }^{2}$ The production of exogastrulæ from sea-urchin blastulæ by adding lithium chloride to the sea water is a similar instance; in this case the endoderm grows outward instead of inward. ${ }^{3}$ The transition is direct from such simple cases of artificial chemical control of development to the cases where various developmental processes occur normally under the control of special chemical substances produced by the organism itself; the influence of hormones illustrates such cases; metamorphosis (in tadpoles), the growth of the skeleton, and the production of sexual characters are thus determined.

In general any condition affecting the rate or character of the formative metabolic reactions has a corresponding influence on growth and development. Such conditions include the influence of physical agents like electricity, light, temperature, contact. It is significant that the term "irritability" is applied, especially in plant physiology, to the susceptibility of growth processes to such modifying influences; in such cases the organism "responds" by changing its rate or manner of growth. Any such response implies a corresponding modification in constructive metabolism; hence such facts show that re-

${ }^{x}$ Cf. Stockard, American Journal of Anatomy, X (I9Io), 369; McClendon, American Journal of Physiology, XXIX (IgI 2), 289.

${ }^{2}$ Cf. the discussion in Child's Origin and Development of the Nervous System, pp. $36 \mathrm{ff}$.

${ }^{3}$ Cf. Herbst, Z. wiss. Zool., IV (I892), 446; Mitteilungen zool. Sla. Neapel, XI (1893), I36. 
sponses involving metabolic synthesis are called forth under the same conditions as the other more familiar types of response, such as muscular contraction in animals, which depends more directly upon processes of metabolic breakdown.

The importance of the relations existing between normal growth and the normal physiological activity of the organism has been hitherto insufficiently recognized. Probably the main reason for this is that in the egg and early embryo the development of any organ up to a certain stage necessarily precedes its functional activity; often, in fact, development is complete before there is any possibility of function (generative organs, many muscular mechanisms). In many other cases, however, normal physiological activity is a prerequisite for normal growth and development. Inactivity means lowered or subnormal metabolism, and this involves subnormal growth; frequently, when physiological activity is subnormal, metabolic construction lags behind destruction, and regression or atrophy ("disusc-atrophy") results. The need of activity for normal growth is most evident in the adult stages of higher organisms, and is especially well shown in intermittently active tissues like voluntary muscle, where increased activity leads to increased growth, as shown in the effects of exercise. while disuse is followed by regression more or less complete. Other tissues show similar conditions; the removal of one kidney is followed by increase in the size of the other, in correlation with the enforced increase of activity; and valvular insufficiency in the heart leads to muscular enlargement. Such cases of compensatory hypertrophy are apparently an example of the above- 
cited general rule, and indicate clearly that the physicochemical conditions determining functional activity are in close relation to those determining metabolic synthesis and growth.

Claude Bernard has pointed out that in any living system a relation of this kind must exist if the system is to persist and retain its normal properties under varying conditions of activity. ${ }^{I}$ All activity involves a certain breakdown of organized structural material, as well as of energy-yielding compounds like sugar; hence a return to the normal or resting condition after stimulation requires that compensatory or constructive processes should be set in motion by the same condition that calls forth the destructive or energy-yielding activity.

The general metabolism of any living system represents an ordered combination of constructive and destructive processes; the living condition always involves metabolic construction; as Bernard expresses it, "synthesis is life," even during rest. Hence the rate of metabolic construction is to be recognized as under the same kind of control as the rate of destruction; i.e., of energy-production or normal activity. Growth processes are therefore modified by any condition (cold, poisons, $\mathrm{H}$-ion concentration, salts, anæsthetics) which alters the general activity of the living cell. The growth of the embryo can be temporarily arrested by anæsthetization; the same is true of seedlings and dividing cells. ${ }^{2}$ Such

' Claude Bernard, Leçons sur les phénomènes de la vie, I, I 27.

${ }^{2}$ Bernard describes the anæsthesia of seedlings and embryos ( $\mathrm{La}$ Science Expérimentale, Paris [I890], p. 224). For a study of anæsthesia of cell-division, see my article in Journal of Biological Chemistry, XVII (I9I4), I2I. 
facts illustrate the unitary character and control of the metabolic processes underlying the various vital manifestations; they show that growth and development are controlled by the same conditions as the other forms of protoplasmic activity. Hence stimulation is a conception which is applicable to growth processes in the same sense as to muscular or nervous activity.

Constructive metabolism thus varies with the general physiological activity of the living system; and this latter activity is determined largely by the external agents which act upon or "stimulate" the protoplasm. The general property of "irritability" thus implies not only the ability of the protoplasmic system to carry out definite reactions in response to stimuli but also the ability to vary its constructive metabolism in correlation with the rate or degree of the energy-yielding or destructive processes. Restitution, compensatory growth, recovery from injury, or fatigue and apparently the normal recovery of the irritable state after stimulation are different manifestations of this constructive process.

\section{GENERAL FEATURES OF STIMULATION PROCESSES}

In general, the term "irritability," as used in physiology, designates the universal property of living matter by which the chemical or other activities of the living system change, in some specific way, in response to changes in the surroundings. We say "change in some specific way", i.e., in a manner distinctive of the living system, in order to separate true cases of stimulation from cases where the chemical or other processes occurring in the protoplasm are changed as a direct consequence of non-vital factors. For example, within the usual 
physiological range $\left(5^{\circ}-40^{\circ}\right)$ a rise of temperature of I0 $0^{\circ}$ ore than doubles the rate of most chemical reactions $\left(Q_{\text {Iо }} 2-3\right)$; this rule applies to many processes which, though occurring within the living system, have in them nothing that is specifically vital; thus the rate of hydrolysis in the digestive tract, the rate of consumption of oxygen or evolution of $\mathrm{CO}_{2}$ by living cells, and the rate of autolysis in dead cells are all accelerated to about the same degree by a given rise of temperature; the same is true of chemical reactions in non-living systems; e.g., the hydrolysis of sucrose by acid. Such accelerations are not instances of stimulation in the physiological sense; true stimulation is illustrated only when the organism, cell, or other living system makes a response whose characteristics can be explained only by reference to the special peculiarities of the system as living. Thus a muscle can be mechanically subdivided by scissors, and the purely mechanical action is the same in the living as in the dead muscle; but, in addition, the former contracts, i.e., exhibits its characteristically vital response. Or a living unfertilized starfish egg or frog's egg mechanically treated in an appropriate way begins a sequence of cell-divisions; the same result follows when a starfish egg is kept at $35^{\circ}$ for 2 minutes, or treated with $\frac{\mathrm{m}}{200}$ butyric acid solution for a similar period.

The change in the behavior of the living system under a given stimulating condition is normally a constant one, but the special nature of this change is determined by the specific organization or "inherited" character of the system, as well as by its physiological state at the time. Hence the same change of conditions acting as a 
stimulus may produce entirely different effects upon different irritable systems, or upon the same system at different times. For example, the same intensity of light will repel one group of animals, and attract another; mechanical treatment may arouse increased activity in one motor organ (a muscle) and inhibit it in another (the swimming plate of a ctenophore). The case just cited is interesting as illustrating another general feature in the behavior of irritable systems; the swimming plates of Mnemiopsis or Eucharis beat rhythmically with considerable regularity, but instantly cease movement when mechanically stimulated in the presence of sufficient Ca salts; e.g., in sea water or artificial media containing calcium; but in similar media containing no calcium, mechanical treatment entirely fails to inhibit the morement, and on the contrary accelerates it. ${ }^{\mathrm{I}}$ This instance shows that the same external change of condition may produce different effects in the same tissue according to its physiological state at the time; under one condition there is an inhibitory, under another an acceleratory response. Electrical stimulation of the nerve supplying a voluntary muscle causes the latter to contract; but the same stimulus applied to the cardiac branch of the vagus nerve inhibits contraction.

Such examples illustrate the distinction between the stimulating effect of an agent or change of condition upon an irritable living system, and the direct effect which it produces by its purely physical or chemical action upon the system. Superposed upon and sequent to the direct physico-chemical effect is the special or physiological effect, the nature of which depends on the

${ }^{r}$ R. S. Lillie, American Journal of Physiology, XXI (1908), 200 
specific vital properties of the system. The given physico-chemical change calls forth or occasions a definite change of activity peculiar to the system. The physiological problem of stimulation has reference to the physical and chemical nature of the conditions under which this specific vital reaction is called forth.

Only a special acquaintance with a given living system or organism can enable us to predict what its behavior will be under a given stimulating condition. Irritability as such, however, is a property which is manifested under comparatively uniform conditions in all organisms; i.e., the tendency to respond to certain kinds of physical change is very widely distributed, if not universal. Such responsiveness is a general character of living matter and is largely independent of special features of structure and organization. Thus apparently all forms of protoplasm are influenced in their activity by the electric current; in many cases-nerves, certain receptors, muscles-very weak currents are sufficient for stimulation; i.e., induce a sudden and profound change in the activity of the system; in other cases the sensitivity to the current is less and the response is more gradual. The electrical sensitivity of living matter is in fact one of its most characteristic peculiarities. Evidently there is some feature of protoplasmic structure or organization, common to all cells and organisms, that renders all responsive to electricity, although in varying degrees. The same is true (though perhaps less universally) of mechanical influences or change of temperature. Chemical sensitivity is also universal; since all living matter depends for its existence upon the incorporation and transformation of the assimilable 
materials present in the surroundings, the existence of a highly developed responsiveness to the external chemical conditions is to be expected. It is especially remarkable that certain groups of compounds-the lipoid-solvent or anæsthetizing group-have a similar reversible depressant action on protoplasmic activities in all organisms, from bacteria to higher plants and animals.

We may class therefore as universal properties of protoplasm: (I) electrical sensitivity, and (2) sensitivity to the presence of special chemical substances in the surroundings. In studying the problem of the conditions of stimulation we are thus brought to consider more especially the reactions of living matter to electricity and to chemical substances in the environment. The fundamental or essential features of protoplasmic structure and composition must be those which determine the special responsiveness to influences of these two kinds. 


\section{CHAPTER IV}

GENERAL PECULIARITIES OF PROTOPLASM AS A PHYSICAL SYSTEM

In considering the general peculiarities of living protoplasm, it is essential to recognize that its characteristic properties and activities depend upon features of composition and structure which are kept in permanent existence only through a continued process of compensation, consisting in the metabolic construction of new and specific compounds to replace those broken down or lost in vital activity. Without this continual automatic renewal and repair the system is an unstable one and cannot persist. Physical diffusion and the normal chemical processes of oxidation and hydrolysis all act toward producing a disintegration of the system; these effects are well seen in experiments on autolysis; the dead cell digests itself and its soluble constituents diffuse into the surroundings. During life the structural and chemical integrity of the system is maintained by means of its continued synthetic activity; the cessation of this activity is the essential change in death.

This general conception of living matter, as a system which holds its own through a balance of constructive and disintegrative processes, is fundamental in physiology. Other systems exhibiting an analogous type of equilibrium, i.e., between constitutive and disintegrative processes, are of frequent occurrence in nature, and have been classed by Ostwald as "stationary systems."'

'Ostwald, Vorlesungen iiber Naturphilosophie, Leipzig (1902), chaps. xii, xv. 
Whirlpools, candle flames, waterfalls are examples. Such systems also exhibit a constant configuration, and are the seat of special activities, in which access of material and energy from without balances or compensates the tendency to disintegration resulting from their own activity and the envirommental influences. As with living organisms, their integrity depends upon continual and balanced interchange with the surroundings. A further general resemblance is that they frequently possess permanent features of form and structure which would be impossible as characters of systems in static equilibrium. Such types of equilibria - in which opposed active processes (rather than opposed pressures. tensions, or potentials) have equal and opposite resultant effects, so that the system as a whole retains constant properties - are often called "dynamic" or "kinctic" equilibria. The possibilities of complex structure, and of correspondingly complex activity, are at a maximum in systems of this constitution; this is readily seen when we contrast a fountain with still water, or a candle flame or fireworks with their components in static equilibrium. We may say that in such systems the possibilities of the fourth or time dimension are added to those of the three spatial dimensions.

Living matter, as a system exhibiting a dynamic equilibrium of the special kind already indicated. exhibits many characteristic peculiarities, both of structure and activity, which are derived from this fundamental feature of its constitution. All living organisms consist largely of structures which would not be possible, as permanencies, if the structural materials were not being continually formed and deposited in such 
a way as to offset the continual breakdown; and these structures subserve or render actual many activities which would be impossible in any other kind of system. In general, the activities which are most characteristic of living as distinguished from non-living systems belong in this class. We may thus understand, on the basis of the general properties of systems in stationary equilibrium, the possibility of the existence of material systems of such complex structure and activity as living organisms. The power of regulation exhibited by stationary systems, i.e., of returning to the original state after disturbance, is one of the chief properties which they exhibit in common with living systems. So long as the constitutive processes continue in action such a result is to be expected. The permanence of such delicate structures as filaments, films, nerve processes, and the other finer products of the formative activity of protoplasm depends on this continual automatic synthesis, which compensates the tendency to physical breakdown.

When any irritable organism or cell responds to stimulation, the energy for the response is derived from the chemical energy of the protoplasmic constituents, usually from the oxidation of carbohydrates. It is clear therefore that one of the essential effects of the stimulus is to alter the rate or character of cell-metabolism. In many cases this effect may be indirect; e.g., in the voluntary muscle cell a large part of the heatproduction following a single stimulus succeeds the contraction $^{\mathrm{I}}$ (Hill); similarly in the turgor-motors of

${ }^{2}$ Cf. A. V. Hill, Journal of Physiology, XLII, XLIV, XLVI, XLVII (IgII-I3); also Ergebnisse der Physiol., XV (I9I6), 340; Physiological Reviews, II (I922), 3 IO. 
plants and possibly in other motile organs. But in all cases the work performed in the response represents energy derived from metabolic breakdown, although this energy may act in the intervals between stimulation by developing a tension or turgor which is released only at the moment of stimulation. A fundamental problem thus arises with regard to the general nature of the conditions in living matter which render its rate of chemical reaction so readily alterable by physical changes in the system.

The most significant general fact is that it is only while the cell is living that its rate of metabolism is readily and quickly changed by a stimulating condition. In general, also, it is only during life that the energyyielding forms of metabolism have a high rate or intensity; typically $\mathrm{CO}_{2}$-production, heat-production, and consumption of oxygen decrease greatly at death, although they may not cease entirely. One of the most remarkable peculiarities of living protoplasm, considered as a chemical reaction-system, is that its chief energyyielding reactions, e.g., oxidation of sugar, proceed rapidly at low temperatures, and in a medium which is approximately neutral. To produce a corresponding speed of reaction in vitro, high temperatures or strong reagents are required. It is probable that the conditions which determine the susceptibility to stimulation are the same as those which are responsible for the high velocity of the energy-yielding reactions. The nature of these conditions is imperfectly understood at present; but apparently they are especially favorable to certain types of oxidation; e.g., of carbohydrates.

The indications are that structural rather than purely chemical factors are of chief importance, since the 
oxidation-furthering enzymes (oxidases) extractable from the cell have a relatively slight influence on the physiologically important oxidations; e.g., of sugar. We may thus regard the possession of a certain type of structure, characteristic of the living state, as chiefly responsible for the facility with which chemical reactions proceed in living protoplasm, as well as for their modifiability under stimulating conditions. The synthetic reactions appear to be largely dependent upon the oxidations; this is indicated by the importance of oxygen for growth processes, as well as by various other facts, although the precise nature of this interdependence is not understood at present. The whole problem of the relations between the structure of protoplasm and its chemical activity is one of fundamental interest, and some of the more general facts and considerations bearing on this problem will now be briefly reviewed.

CHEMICAL REACTIVITY OF IIVING MATTER AS RELATED TO STRUCTURE

All living matter is characterized by the possession of a certain structural organization or permanent arrangement of components which is essential to its normal activity. If we destroy protoplasmic structure by heat, mechanical injury, or chemical treatment, the specific metabolic activity of the system and its responsiveness to stimulation are lost.

In general, the chemical reactions of living matter may be grouped under two classes according to their relation to protoplasmic structure: (A) those reactions which continue in an essentially unaltered manner after the "life" of the cell has been destroyed; e.g., in cell- 
extracts or in the residue remaining after complete mechanical or other disintegration of the protoplasm; and (B) those which continue only while the protoplasm remains structurally intact and "living." The fonner group (A) includes a large number of hydrolyses and some oxidations; e.g., those due to oxidases; but, as already indicated, the physiologically significant oxidations, especially of sugar and other energy-yiclding compounds, cannot be accomplished, at least with anything like the normal velocity and completeness, under the influence of enzymes or cell-extracts. Yeast cells which have been mechanically destroyed, or even simple watery extracts of yeast, rapidly hydrolyze cane sugar, just as does the living cell, and autolyzing yeast cells split proteins rapidly into amino-acids. It has been found, however, that the alcoholic fermentation of sugar proceeds much more slowly in the press-juice of yeast than it does under the influence of the living protoplasm. ${ }^{3}$ Many other cases are known where biochemical reactions, although proceeding in dead cells or under the influence of cell-extracts, do so at a slower rate than in living protoplasm.

The latter group of reactions (B) include the specific syntheses, i.e., of protein, together with those syntheses which require the expenditure of considerable energy: like the building up of fats from carbohyclate, or of amino-acids and other compounds of high chemical potential from compounds of lower potential. The energy required for these syntheses is apparently de-

${ }^{2}$ Cf. Harden, "Alcoholic Fermentation," in Monographs on Biochemistry, edited by Plimmer and Hopkins. 
rived from the oxidation of other compounds, especially carbohydrates. ${ }^{\mathrm{T}}$

It is especially significant that in all cases the synthesis of specific proteins, the reactions essential to growth and maintenance, requires the intact protoplasmic structure. These syntheses constitute the chemical reactions most highly characteristic of the living state. At one time it was believed that all of the metabolic syntheses were the result of the special activity of living protoplasm, and that the chemical reactions of dead protoplasm or cell-extracts were always of a catabolic (splitting) kind; it is now known, however, that various syntheses involving little change of energy, e.g., the synthesis of esters and disaccharides, readily occur under the influence of enzymes alone. Yet the fundamental fact remains that the more important or specific part of the synthetic activity of protoplasm is exhibited only during life. A relation of the normal protoplasmic structure to certain types of chemical action, especially synthetic action, is thus indicated. With the alteration of structure occurring at death, as indicated by loss of semi-permeability, coagulation of cell-proteins, and other phenomena of disintegration, is associated a loss of synthetic power. Only the living yeast cell can build up from a solution of sugar, tartrates,

${ }^{x}$ Hence the importance of carbohydrates for growth and assimilation; e.g., in plants, carbohydrate is indispensable for the assimilation of amino-acids by yeast and molds (cf. the series of papers by F. Ehrlich, Biochem. Zeitschrift, I, VIII, XVIII, XXXVI (Igo6-II); similarly in higher plants the synthesis of proteins from amides in germination requires the presence of carbohydrates (cf. Jost's Physiology of Plants, p. 175 , for a summary of the chief facts). The sequence of metabolic derangements associated with diabetes shows the fundamental importance of carbohydrate metabolism in higher animals. 
and inorganic salts the various special compounds, definite and constant in number, proportions, and distribution, which compose the yeast protoplasm.

Some of the changes in the chemical reactivity of protoplasm resulting from mechanical or other destruction of the living cells or tissue are well illustrated by Fletcher's and Hopkins' work on the formation and disappearance of lactic acid in muscle; ${ }^{\mathrm{I}}$ also by the work of Harden and Maclean on oxidation by isolated animal tissues; and more recently by Warburg's determinations of the oxygen consumption in living cells (sea-urchin eggs, blood corpuscles, bacteria, etc.) as compared with that of the same cells after death or fine mechanical subdivision. $^{3}$ In all of these cases chemical activity is greatly decreased when the protoplasmic structure is artificially destroyed. Warburg has also shown that when certain cells, the blood corpuscles of birds, are mechanically broken down by freezing and thawing, the oxygen consumption exhibited by the residue is associated with the more solid part of the complex that which can be separated by centrifuging; similarly, in liver cells the separable granules have a relatively high oxygen consumption. ${ }^{4}$ A relation of oxidative activity to the solid part of the protoplasmic structure is thus indicated. In some cases it can be shown microchemically that certain oxidations (the indophenol reaction) occur most actively at the surfaces of solid

'Fletcher and Hopkins, Journal of Physiology, XXXV (1907). 247.

${ }^{2}$ Harden and Maclean, Journal of Physiology, XLIII (1011), 34.

3 Warburg, Ergebnisse der Physiol., XIV (1014), 253.

4 Warburg, cf. Biochem. Zeitschrift, CXIX (1021), 134, and references to earlier papers there given. 
structures, such as the nuclear and plasma membranes. ${ }^{\mathrm{I}}$ It is interesting to note that a visible alteration or breakdown of protoplasmic structure seems always to be associated with the death process, however induced; even after natural death, coagulative or other alterations occur in most forms of protoplasm; death rigor, increased permeability, and loss of tensile strength in muscle cells, are examples of such effects. The death change involves a structural disintegration, with which is associated a loss of normal chemical activity.

A second class of cases, in which certain chemical reactions may be promoted instead of hindered by the breakdown of normal cell structure, also throws light upon the relation of structure to the chemical activity of protoplasm; an example is the autolytic breakdown of proteins or of glycogen in dead liver cells or other autolyzing cells. The rate of such breakdown is increased when the structure is altered by death, and still more so (according to Chiari's observations) in the presence of lipoid-solvent compounds like chloroform. ${ }^{2}$ Such facts illustrate another form of chemical control exercised by protoplasmic structure. Apparently they indicate that a partitioned or alveolar structure exists during life; enzyme and substrate, for example, may thus be kept apart while this structure is intact, but on death the interalveolar partitions are broken down and interaction results. It has been suggested by Hofmeister ${ }^{3}$ that this chambered type of architecture is what renders it possible for a variety of chemical reactions to occur

${ }^{I}$ R. S. Lillie, Journal of Biological Chemistry, XV (I913), 237.

${ }^{2}$ Chiari, Arch. exper. Path. Pharmakol., LX (I909), 256.

${ }^{3}$ Hofmeister, loc. cit. 
within the limits of a single cell without mutual interference; different metabolic processes are thus localized, a necessary condition for a definite "chemical organization" of the cell.

It is well known that when living protoplasm is acted upon by cytolytic agents or heat $\left(40^{\circ}\right)$ or is altered mechanically or osmotically (i.e., by hypertonic or hypotonic media) beyond a certain degree, chemical changes are induced in it which are absent or inappreciable under normal conditions. These changes are associated with profound structural alteration, as shown by coagulation of the cell-proteins, changes of permeability and water content, and loss of the normal tensile and other mechanical properties of the protoplasm. Thus in muscle, and probably in most other cells, lactic acid is formed in large quantity; in many cells autolytic changes are initiated; in oxidase-containing fruits and tubers (apple, potato) the browning reaction occurs; and in many cases (muscle) there is a marked temporary increase in the output of $\mathrm{CO}_{2}$. Of special interest is the fact that these changes are associated with a loss of the normal semi-permeability of the plasma membranes, coincidently with a loss of the characteristic water-immiscibility of the protoplasm as a whole; hence disintegration by diffusion processes follows rapidly: These effects are such as might be expected to result from a breakdown of the normal partitioned structure of the system. Materials which during life are $k e p t$ apart by the interposition of films are thus enabled to interact; hence (as already cited) autolysis is accelerated by cytolytic compounds like chloroform. For a similar reason the minuter structural elements - which nomally 
are prevented by the pervading film-structure from fusing or otherwise losing their identity-undergo alteration or breakdown; a general coarsening or increase of opacity, indicating coagulative changes in the cell proteins, is characteristic of dying protoplasm, and is associated with the changes in mechanical properties described above. ${ }^{\text {T }}$

The basis of these changes is insufficiently understood at present, but their ready production by lipoid-solvent compounds seems to indicate that the lipoid constituents of the protoplasm are specially involved. Apparently the lipoids have a relation to the protein constituents resembling that which a "protective colloid" (gelatine) added to a suspensoid hydrosol (gold) has to the colloidal particles of the suspensoid. In the presence of the protective substance the particles remain separate under conditions, such as the presence of salts or increase of $\mathrm{H}$-ion concentration, which otherwise lead to fusion or precipitation; ${ }^{2}$ this stabilizing influence is apparently dependent on the formation of thin adsorption films about the particles. In the case of living protoplasm, the evidence from cytolysis and similar phenomena indicates that the normal fine subdivision of the structural proteins-shown by the characteristic translucency during life-is dependent on the presence of thin lipoid films (possibly soap) at the surface of the protein particles, fibrils, or other structural elements. When these films are broken down or destroyed, a coalescence of particles and a coarsening of structure result; these effects involve a loss of semi-permeability, together with the changes

${ }^{x}$ The progress of structural changes of this kind can be followed by the microscope under dark ground illumination; cf. Aggazzotti, $Z$. allg. Physiol., XI (I9I0), 249.

${ }^{2}$ Zsigmondy, Colloids and Ultramicroscopy. 
in mechanical and chemical properties already described. On such a view the cytolytic action of lipoid-alterant compounds may be explained. Such compounds act by destroying the film-structure; hence, in addition to destruction of semi-permeability, they break down the intracellular partitions and induce chemical reactions of the above-described kind and cause coagulation of the cell-proteins. In irritable cells such compounds have also a strongly stimulating action of an irreversible kind, as shown in the contraction produced in muscle cells, and similar effects.

The above-described loss of translucency accompanying cytolytic or mortiferous processes is a phenomenon of much interest, which has an intimate bearing on the general problem of protoplasmic structure. This change is shown with great clearness in all of the more transparent forms of protoplasm; e.g., the eggs of marine animals (starfish, etc.), protozoa, and muscle cells. Some years ago while studying the conditions of activity in the ctenophore swimming plate - a beautiful example of a clear translucent protoplasm, consisting of parallel contractile fibrils (fused cilia) - I was struck with the constancy and definiteness of the relations existing between changes of translucency and changes of contractile activity. In dying animals the plates become partially clouded and adopt a rapid unintermittent movement, differing from the normal movement in being of quicker rhythm and in no longer showing the mechanical inhibition described above; this movement continues until finally the plate becomes white and opaque and all activity ceases. ${ }^{x}$

${ }^{x}$ R. S. Lillie, American Journal of Physiology, XVI (1906), 117 ; XXI (1908), 200. 
A similar cycle of alteration is passed through, only more rapidly, when the normal plates are transferred from sea water to various unbalanced solutions, such as pure isotonic $\mathrm{NaCl}$; the plates then exhibit for a brief period (one or two minutes) an extremely active vibratory movement, which is associated with a progressive whitening or coagulation. In general the rate of coagulation is more rapid the more energetic the contractile activity; and it is especially noteworthy that the coagulative process does not begin until the plate starts vibrating; the vibration then continues until the whole structure is opaque. This change of structure is irreversible, and at the end the plate is so altered in consistency that it readily falls to pieces when shaken. Evidently the contractile activity is associated with the removal of some substance or condition which prevents the coalescence of the protein particles forming the fibrils. A film-structure of the kind suggested above seems indicated, which is broken down by the action of the solution with the production of both chemical and mechanical effects. The general relations between such effects and stimulation processes will be considered in more detail below. Apparently in the swirnming plate the essential effect produced by the unbalanced solution is an acceleration or intensification of the normal processes of stimulation and contraction; a dependence of these processes on the alteration or removal of film material is thus indicated. The indications are that during the normal rhythm of contraction in sea water the film-structure is alternately broken down and reformed in each contractile cycle. Presumably under the abnormal conditions resulting from the action of the pure $\mathrm{NaCl}$ solution the rate of 
breakdown is increased, and the restoration of filmstructure between successive contractions becomes imperfect, with the result that eventually the whole structure disintegrates. These effects may be compared with those of excessive fatigue, which also leads to irreparable structural breakdown. ${ }^{\text {I }}$

\section{STRUCTURE OF PROTOPLASM}

It will be evident from the preceding discussion that structure is only one factor in the chemical activity of protoplasm; undoubtedly many other factors - those entering in all chemical reactions, such as concentration, temperature, special affinities, catalysis-enter in determining the rate and character of the metabolic reactions. But the controlling factor-that which is subject to rapid and reversible alteration under the influence of stimulating agencies - appears to be the peculiar structure of the living substance. By the conception of "structure" as applied to protoplasm is meant, generally" speaking, the distribution of the physically stabler components, usually the solid components, of the system. Evidently, as already pointed out, this structure is itself a product of metabolism; but having once been formed, it influences the further course of metabolism - in the general manner of which Child's comparison of the living organism to the flowing river $^{2}$ gives a good illustration by analogy. In the living organism there is always structure of a definite kind; even the simplest "undiffer-

${ }^{x} \mathrm{Cf}$. the instances of structural alterations in the central nervous system described in Crile's recent book, A P'hysical Inlirpritution of Shock, Exhaustion, and Restoration, London (I92I).

${ }^{2}$ Cf. Child, The Regulalory Process in Organisms, Journal of Morphology, XXII (rgIr), I7r; also Senescence and Rejuicnescence, chip. i. 
entiated" protoplasm is not homogeneous, and it is necessary to reach a clear conception of the essential nature of this structure in the most generalized forms of living substance if we are to be in a position to understand the fundamental conditions of physiological activity.

That metabolism is controlled by structure is seen in many well-known physiological facts already referred to in part; e.g., the course of development, with the associated constructive metabolism, may in many eggs or embryos be profoundly modified by artificially altering the structure of the system. Developmental processes are frequently initiated by mechanical means; cases of regeneration illustrate this, or cases where injury of the egg-surface (pricking in the case of the frog's egg, ${ }^{\mathrm{T}}$ or any kind of cytolytic action in echinoderm eggs) ${ }^{2}$ initiates cleavage and development. Mechanical treatment causes stimulation in innumerable instances; in others it causes inhibition. In all of these cases the energy for the developmental or other response comes directly or indirectly from metabolic processes. This sensitivity to the action of mechanical agents, which by their impact, pressure, or other effects locally modify cell structure, is perhaps the clearest proof of the intimate relations existing between structure and function in living protoplasm.

In physical chemistry the importance of structural conditions as modifying factors in chemical reactions is illustrated in the so-called heterogeneous catalyses. In these phenomena the acceleration of reaction is

${ }^{x}$ Guyer, Science, XXV (1907), 9ro; Bataillon, Arch. zool. expêr. et gênérale, XLVI (I910), то3.

${ }^{2}$ Loeb, Artificial Parthenogenesis and Fertilization, University of Chicago Press (19r3). 
dependent chiefly on surface effects, of which two classes appear to be especially important from the biological point of view: (I) adsorption effects, leading to increased concentration at surfaces and hence increased reactionvelocity; and (2) electrolytic effects, due to the existence of local potential differences between different regions of the surface separating the two phases; when both phases conduct electricity, local circuits may thus arise, furnishing the conditions for electrolysis. This latter effect may also be regarded as a form of catalysis, and is illustrated in the spreading of rust spots on iron surfaces, or the periodic catalysis of $\mathrm{H}_{2} \mathrm{O}_{2}$ by mercury. Both kinds of effects are of fundamental importance in protoplasmic processes, as will be shown in more detail later. Other conditions characteristic of surfaces may also enter (see pp. 2I 7 ff.).

As already pointed out, all forms of protoplasm exhibit the power of specific synthesis characteristic of life. The constructive metabolism by which the specific structural elements are built up and maintained must. like other forms of metabolism, be under the control of structure. This synthetic activity, being a universal property of living matter, is undoubtedly to be correlated with the most general or fundamental type of structure exhibited by protoplasm. In correspondence with its uniformity of essential chemical composition and chemical behavior, protoplasm must also possess a uniformity in its essential type of physical structure; underlying the variety of structural detail must be some characteristic type of structural composition common to all forms of protoplasm, and determining the special features of its chemical activity. The traditional problem of the 
structure of protoplasm is thus intimately bound up with the basic problem of general physiology.

The problem relates to the nature of the structure in living protoplasm. All observers agree that cell structure is profoundly altered by death; disintegration then follows, accompanied by diffusion of the cell constituents into the surrounding medium. As already described, the death of the cell is associated with loss of its normal osmotic properties or semi-permeability; the normal electrical polarization also disappears at the same time; both phenomena are characteristic, and indicate interruption in the continuity of the protoplasmic boundary layer. The most obvious general structural changes occurring in the cell interior at death are of a coagulative kind; the protoplasm loses its normal translucency and becomes more opaque (death rigor or death coagulation). This effect is seen in the greatest variety of cells and organisms, especially those with translucent protoplasm, as cited above. The protoplasm of muscle cells becomes more opaque and loses its coherency or tensile strength; dying swimming plates whiten and fall to pieces on shaking, and other phenomena of a similar kind are well known to all biologists. Many observations on the postmortem alterations of structure have been made since the introduction of the methods of microdissection. Kite and Chambers describe dying cells as losing their viscidity and as being easily torn to pieces. Chambers describes the isolated nerve-ganglion cells of the lobster as undergoing irreversible structural changes when mechanically injured; the protoplasm then "sets into a coagulated non-viscous mass which may be broken into non-glutinous pieces." Taylor describes a similar break- 
down of protoplasmic structures in Protozoa after injury with the microdissection needle. ${ }^{\mathrm{I}}$

Facts of this kind show again that the maintenance of a certain characteristic type of structure is an essential part of normal protoplasmic activity. The structure of living protoplasm is not to be conceived as resulting from a combination of static parts like the structure of a machine; it is the product or expression of continual synthetic activity and persists only while metabolism persists; it expresses the constructive activity of metabolism, very much in the same manner as the structure of a flame or of a fountain expresses the dynamic activity of such a system. If the activity disappears, so also does the characteristic structure or configuration which is maintained by that activity. In this sense, structure in living protoplasm is to be conceived as continually in process of formation; i.e., as an index of the underlying synthetic reactions which, as already seen, are inseparable from the chemical activity of the system during life. The apparently static condition represents in reality a state of balance between construction and disintegration.

Yet a certain permanent or stable structural constitution (at least relatively permanent) has to be assumed. just as in the case of the fountain or candle flame. This is necessary if the dependent processes of chemical transformation are to exhibit constant characters. The physical nature of this permanent or persistent structural substratum of living protoplasm has first to be considered.

${ }^{I}$ Kite and Chambers, Science, XXXVI (1912), 640. Chambers, Trans. Royal Soc. Can., XII (I9I8), Series 3, 43; Taylor, L'niversity of Califormia Publications, XIX (1920), 403, cf. pp. 420, 424, 434. Mention has already: been made of Aggazzotti's observations with dark ground illumination on the structural changes produced in blood corpuscles by cytolytic agents . Cf. also Traube and Klein, Biochem. Zeits., CXXX (1922), 477. 


\section{CHAPTER V}

PHYSICAL NATURE OF PROTOPLASMIC STRUCTURE: IMPORTANCE OF SURFACE CONDITIONS

It is not possible here to review in detail the numerous and frequently conflicting conceptions of protoplasmic structure. The details made visible by microscopical technique are of so varied a kind that none of the many attempts at unification have met with universal agreement. A chief difficulty has been that most histological investigators seem to have conceived of protoplasmic structure as existing independently of the chemical and physiological activities of the living system, and not as both dependent upon and determining these activities. Some conception of structure is required which will be general enough to apply to all of the forms of living matter, and which will at the same time enable us to understand the dependence of the fundamental vital properties of specific synthesis and irritability upon structure. It may be doubted whether we are yet in a position to form a clear and permanently valid conception of protoplasmic structure, but with the progress in our knowledge of the properties of colloidal systems has come what appears to be an increased insight into the possibilities. The problem may be defined in its essential terms, as follows: Can a system, with components of the kind which we find present in all living matter, be imagined which will exhibit, as a correlative of its structural composition, the above-described properties of specific growth, sensitivity to electrical conditions, catalytic 
activity, and automatic regulation of composition and properties?

The experimental studies and observations of the last twenty years have led more and more to the conclusion that the general or fundamental structure of protoplasm corresponds more closely to that of an emulsion than to that of any other simple non-living physical system. The most general facts of its chemical composition are in agreement with this conclusion. Water-insoluble constituents (lipoids) occur in association with colloidal constituents which have water-combining powers (proteins). The whole resulting complex is during life immiscible with water, and typically is bounded from the external watery medium forming its immediate environment by a layer or surface-film having semipermeable properties. The semi-permeability and the water-immiscibility of the surface layer appear to be interdependent properties; they suggest the existence of a continuous external layer of water-insoluble material of fatty or similar nature. ${ }^{x}$ The unit of organic structure, the cell, would thus appear to be a sy'stem with an aqueous internal phase limited externally by a thin water-insoluble phase or boundary layer. The aqueous internal phase forms one component of a system, the cell protoplasm, which is structurally and chemically highly complex, and emulsion-like in its general physical constitution.

It is evident that the general properties of emulsions do not in themselves explain the properties of living matter. What seems highly probable, however, is that the original structural foundation upon which the proper${ }^{x}$ Cf. Quincke, Ann. Physik., XXXV (I8S8), 580; cf. pages 629-30. 
ties of living matter have arisen-or which has made it possible for systems with vital properties to evolveis that of an emulsion; i.e., a polyphasic system with thin interfacial films separating two or more component phases which have fluid or solvent properties. From general considerations it seems clear that some kind of polyphasic structure must be assumed in order to account for such a universal property as that of growth; the unit of living matter, even while it continues to increase in size, retains a complex and specific composition different from that of the surroundings; and this peculiarity is in itself incompatible with structural homogeneity, since the elementary need of providing against free diffusive interchange with the surroundings requires a surface layer with properties different from those of the internal protoplasm. This must be true even of the simplest forms of living matter. We cannot compare the protoplasm of ultra-microscopic organisms with self-propagating enzyme-like material (supposing such material possible), as has been done, since the physical conditions necessary for metabolism and growth must exist in even the simplest living systems; and this requires at the very least a differentiation between the more permanent or solid components of the system and the liquid components which contain in solution simpler materials (nutrients and oxygen) which are continually being renewed.

It has long been recognized that colloids form the basis of protoplasmic structure. Hardy's investigations showed that many of the characteristic structural appearances presented by fixed and stained protoplasm in microscopic preparations were incidental consequences 
of the colloidal composition of the system and not expressions of any distinctively vital condition or structure. He and others have demonstrated that similar appearances can be produced by fixation in apparently homogeneous colloidal solutions or gels (egg-white, gelatine). Hardy also pointed out various parallels between the processes of gelation in artificial colloidal systems and the changes of physical state in living protoplasm. ${ }^{2}$ From these and related facts it became clear that if we are to draw conclusions regarding protoplasmic structure from the appearances seen in microscopic preparations, the general nature of the changes produced in colloidal systems by physical and chemical agents must first be determined. Great impetus was thus given to the study of the physics and chemistry of colloids, a subject then in its early stages, and also to the study of the structure and physical properties of protoplasm in the living condition.

Various resemblances between living protoplasm and emulsions were long ago described by Bütschli. ${ }^{3}$ 'These resemblances relate both to structure and to certain peculiarities of behavior; e.g., amoeboid movement and modifications of activity by changes in the surroundings. Bütschli reached the conception that a "foam structure," corresponding essentially to a filmpervaded or chambered structure, is the type most generally exhibited by living protoplasm. ${ }^{4}$

IW. B. Hardy, Journal of Physiology, XXXV (Isyg), 15 \&; cf. also Alfred Fischer, Fixierung, Färbung, und Bau des Proloplasmas, Jena ( I Sog).

${ }^{2}$ Hardy, Proceedings of the Royal Socicty, LAVI (IS99), 110.

3 Bütschli, Microscopic Foams and Proloplasm.

${ }_{4}$ Cf. the discussion by E. B. Wilson, Jour. Morph., XI (1Sgo), Supplement. 
The subject of the physical chemistry of emulsions forms a part of the now extensively developed field of colloid chemistry, and cannot be considered here in any detail. Some of the more general facts relating to the structure and properties of emulsions must, however, be discussed briefly, since a clear conception of the physical conditions existing in these systems is necessary before proceeding to the consideration of the more complex types of structure and behavior which have evolved in living matter, apparently with emulsionsystems of a relatively simple kind as a basis.

\section{EMULSIONS ${ }^{\mathrm{I}}$}

Emulsions and foam structures are essentially similar systems, with the difference (as usually defined) that in a foam the disperse or discontinuous phase is a gas, in an emulsion a liquid. Jellies or gels also resemble these systems in constitution in many cases. It is now known that various different types of gel structure exist; many jellies, however, are essentially dense emulsions; thus stiff foams of air with a soap solution (or solutions of albumin, saponin, or other surface-active colloidal substances) have many of the characters of solids or semi-solids; i.e., permanence of form, elasticity, high viscosity; and all transitions between liquid and solid systems of the emulsion type are known-a fact familiar to anyone who makes a lather of soap solution. The same is true of an emulsion of one liquid in another; thus an emulsion of oil in a soap solution may be made

${ }^{x}$ For a general review cf. Bancroft's series of articles on "The Theory of Emulsification" in Journal of Physical Chemistry, XVI-XIX (19I2-I5); also the recent book of Clayton, The Theory of Emulsions and Emulsification. 
so concentrated - with more than 90 per cent of oil as a disperse phase in some of the emulsions prepared by Pickering ${ }^{\mathrm{x}}$ - that the whole mass has a jelly-like consistency. The differences of opinion as to whether living protoplasm belongs to the "sol" or "gel" type are thus seen to be unimportant, since transitions between these states are continuous, and in fact many forms of protoplasm exhibit a liquid consistency at one stage (or under certain conditions) and a solid consistency at another. ${ }^{2}$

In a foam of air and soap solution the individual bubbles do not coalesce, although the intervening films may be extremely thin; evidently the structural stability of the system as a whole is determined by the properties of the films. If we break down these films, mechanically or otherwise, the whole foam structure collapses. The stability of emulsions of oil in aqueous media is similarly conditioned; in this case the coalescence of the separate oil droplets is prevented by interfacial films of soap or other material, and such an emulsion can be also destroyed (de-emulsified) by altering the material composing the films, e.g., by adding strong acid if soap is the emulsifying material. Such facts lead to the general question of the conditions determining the stability of a foam structure or emulsion.

Two chief conditions for the persistence of an air foam are that the layer of solution separating the adjacent bubbles should have (I) a low surface-tension and (2) a high viscosity. A low surface-tension is favorable because the tangentially acting forces tending

'Pickering, Journal of the Chemical Society, XCI (1907), 2001.

${ }^{2}$ Cf. Bayliss' recent paper in Proceedings of the Royal Sociely; B, XCI (1920), I96. 
to rupture the films or lamellæ are then small; i.e., the natural tendency of the film material to minimize its surface, or "draw together," is slight. But this condition is not alone sufficient, as seen in the fact that pure liquids of low surface-tension against air, like ether, benzol, alcohol, etc., do not give permanent foams, any more than does water. It is well known that mixtures of alcohol and water foam more readily than either liquid alone; and this is especially true of mixtures of water and a second liquid of great surface-activity and high viscosity, such as amyl alcohol; hence the important generalization that pure liquids do not foamdo not form permanent disperse systems with air. Nor do mixtures of two pure, mutually immiscible liquids readily form permanent emulsions. Typically the presence of a third substance is necessary, and it is important that this third substance should be of such a kind as to lower the surface-tension at the boundary between the phases, and also to impart to the surface layer a relatively high viscosity or resistance to displacement. Under some conditions this viscosity may be sufficient to impart to the interfacial layer the properties of a solid film. In general, a third substance is effective as an emulsifying agent in proportion to its power of forming at the boundary a film having these properties of low surfacetension and high viscosity. Most substances which form stable emulsions of oil in water (soap, proteins, gums) are of this kind. The interfacial films or lamellæ then resist disruption and the disperse droplets are prevented from fusing. If the film is considered as a phase, most emulsions would be classed as three-phase systems (triphasic). 
Another condition faroring stability in an cmulsion is a small diameter in the disperse droplets. The disperse particles in suspensions and emulsions are electrically charged, and if they are sufficiently minute the forces due to their mutual electrostatic repulsion may be sufficient to prevent contact and fusion. ${ }^{\mathrm{I}}$ We must therefore qualify the statement that at least three components are necessary in a permanent emulsionsystem by the proviso that the subdivision be not excessively minute. This factor, however, is of minor importance in most emulsion systems, and probably is not of great importance from a biological point of view. It is worthy of note, however, that in some cases mutual electrostatic repulsion appears to play a part in determining the distribution of colloidal particles, droplets, or other minute freely mobile particles in cells; e.g.. the distribution of the chromatin in the equatorial plates and spiremes of mitotic figures shows evidence of this factor. ${ }^{2}$

An emulsion, being a system of disperse charged particles, resembles in this respect any colloidal suspension; hence electrolytes influence the stability of emulsions, because of the influence of the ions on the interfacial potentials, just as they influence the stability of other colloidal systems. ${ }^{3}$ Generally speaking, any mechanical, chemical, or electrical conditions which alter the surface lamellæ affect the stability and other

${ }^{2}$ Cf. Lewis, Kolloid-Z., V (1909), 9 г.

${ }^{2}$ R. S. Lillie, American Journal of Physiology, XV (1,06), 46.

${ }^{3}$ For the action of electrolytes on the stability of emulsion. of Powis, Z. physik. Chem., LXXXix (191-15), 186. (f. Northrop and De Kruif, J. Gen. Physiol., IV (1921-22), 030, for an account of the analogous action of electrolytes in the agglutination of bacteria 


\section{PROTOPLASMIC ACTION AND NERVOUS ACTION}

properties of an emulsion system. Emulsions of oil in alkaline water or soap solution are destroyed by adding strong acid $(\mathrm{HCl})$ which breaks down the soap films. Similarly a foam structure may be destroyed mechanically or by adding a surface-active substance of low viscosity; thus a few drops of ether destroy a beer-foam, a fact explained by Quincke as due to the displacement of the material composing the surface lamellæ. ${ }^{x}$ Similarly a saponin solution to which sufficient alcohol is added does not form a permanent foam; the addition of isobutyric acid to a saponin solution also prevents foaming, but if alkali is added to neutralize the acid and form the surface-inactive salt, foaming results. ${ }^{2}$ Many other facts of a similar kind are well known. The conditions of de-emulsification ("cracking" of emulsions) deserve careful study by biologists, for changes of this kind are almost certainly concerned in many forms of protoplasmic activity; e.g., secretion and the processes of activation, stimulation, and cytolysis.

In general, therefore, we may define the chief condition of stability in emulsion systems as the presence of material, differing from that composing the two chief phases, in the form of thin continuous layers or films deposited or adsorbed at the boundary surfaces. The thickness of these films may be extremely slight; when a material is surface-active and is free to spread over the surface separating the phases, conditions of equilibrium may not be reached until the layer is only one or two molecules thick. ${ }^{3}$ Such a film, however, is

${ }^{x}$ Quincke, Ann. Physik, XXXV (1888), 580.

${ }^{2}$ Zawidski, Z. physik. Chem., XXXV (I900), 77.

${ }^{3}$ Cf. Langmuir, Journal of the American Chemical Society, XXXIX (I9I7), I848; cf. also Freundlich's Kapillarchemie, p. 278. 
capable of holding one liquid finely dispersed in another in a permanent state of emulsion. We may infer that in at least some forms of protoplasmic emulsion-structure the interfacial films are of molecular thickness, a consideration of much importance in relation to the properties of irritability and transmissivity (or propagation of excitation-states), as will be seen below.

\section{ADSORPTION}

It will be clear from the above that in the formation of emulsions - and hence of living protoplasm as a system based upon the emulsion type of structure-the conditions determining the formation of interfacial films are of primary importance. Adsorption, the process by which material collects or concentrates at boundary surfaces, is thus a fundamental factor in the formation and behavior of emulsion systems and of colloidal systems in general. The physics and chemistry of adsorption processes have recently been discussed fully in several excellent textbooks, ${ }^{1}$ so that it is unnecessary here to give any detailed account. One general fact, however, which may be emphasized as especially important from the physiological point of view, is that adsorbed substances are typically more subject to chemical change than substances uniformly distributed in a solution. Both the increase of concentration and the presence of surface factors are concerned in the increase of reactivity, the catalytic action of many finely divided materials (charcoal, platinum, etc.) is usually referred to the increased concentration of the chemically altered material

${ }^{1}$ Höber, Physikalische Chemie der Zelle und der Gewebe (101\$); Freundlich, Kapillarchemie, Leipzig (1909); Bayliss, Principles of General Physiology; Bancroft, Applied Colloid Chemistry. 
at the surface of the catalytic agent, but it appears probable that other factors (electrical) also enter in many cases of adsorption-catalysis (see below).

In considering the case of protoplasmic systems, we may regard adsorption as of importance in two chief respects: (I) as an essential condition in the determination of structure (through the formation of the adsorption-films of the protoplasmic emulsion and in membrane structure in general), and (2) as a main factor determining the character and velocity of the chemical reactions; i.e., as influencing or controlling cell-metabolism.

It is well known that the adsorption of dissolved substances of low molecular weight is, as a rule, a strictly reversible process, with the equilibrium conditions defined by the formula $x / \dot{m}=k c^{\frac{x}{n}}$, where $x$ is the quantity adsorbed, $m$ the mass of the adsorbent, $c$ the concentration of the substances in solution, and $k$ and $n$ constants. On the other hand, in the case of colloidal substances or other substances of high molecular weight, adsorption frequently leads to a change of properties, the substances becoming converted into relatively insoluble or resistant varieties $^{\mathbf{I}}$ (possibly polymerized). In such cases the process may be difficultly reversible or irreversible, a fact of much interest as bearing on the question of the conditions under which the more permanent portion of the protoplasmic substratum is formed. In general, organic growth appears to depend on the deposition of relatively stable or persistent structural elements or material in apposition to other elements or material of

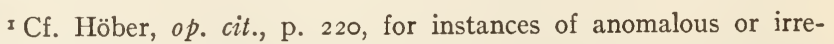
versible adsorption. 
the same kind, a process suggesting an irreversible type of adsorption. A few examples of irreversible adsorption may be cited for illustration. Freundlich and Losev $^{\mathrm{I}}$ found that a solution of the dye, crystal violet, was completely decolorized by animal charcoal, and that washing would not give back the dye. This may mean that the concentration of the dye in solution at equilibrium is indefinitely small; but more probably. it points to the formation of an insoluble modification as the result of adsorption. Proteins like egg-albumin when adsorbed at the surface of drops of chloroform, form thin highly insoluble and resistant pellicles; i.e., the protein undergoes the change usually described as "denaturation." Various other cases of anomalous adsorption are probably to be referred to conditions of a similar kind; the adsorbed material apparently undergoes some chemical modification.

A further fact of fundamental biological interest is that the adsorbent action of many materials has a certain specificity or selective character; i.e., the action varies from adsorbent to adsorbent independently of the latter's state of subdivision. This phenomenon has apparently the same ultimate basis as have the specific chemical affinities between substances; in fact, the distinction between adsorption and the formation of true chemical compounds is now very generally recognized as ill defined. ${ }^{2}$ The phenomena of cohesion, adhesion, and capillarity are closely related to adsorption; thus water wets (is adsorbed by) certain solid surfaces,

${ }^{x}$ Freundlich and Losev, Z. physik. Chem., XIIX (1907), $2 S_{4}$.

${ }^{2}$ Cf. Langmuir, op. cit., pp. I 900 ff.; also Journal of the American Chemical Sociely, XL (1918), 136r. 
but not others. It is well known that the interfacial tension between an adsorbing surface and a solution of an adsorbable substance is a direct function of the degree of adsorption of the latter.

When the adsorbed substances are of low molecular weight-e.g., in homologous series of alcohols, organic acids, or similar compounds - it is usually found that the order of relative adsorption is not altered by altering the adsorbent, although the degree of adsorption may vary widely with the different adsorbents. With more complex molecules, however, relations of an apparently arbitrary or specific kind often enter, and presumably the relations between the molecular structure or configuration of the adsorbing surface and that of the adsorbed substance then become important. Specific adsorptions, like specific chemical combinations (between enzyme and substrate, or antigen and anti-body) are thus probably largely dependent on similarities of chemical configuration. Hence a good adsorbent for one substance may be a poor one for another. ${ }^{I}$ Freundlich cites various instances illustrating the differences between the adsorbent powers of different materials for the same substance. ${ }^{2}$ Thus charcoal adsorbs crystal violet 20 times as effectively as silk, and $5_{5} 6$ times as effectively as cotton wool. He found that different adsorbents usually showed the same order of relative adsorption for the dyes used; with four solid adsorbents the general order of adsorbent action was charcoal $>$ wool $>$ silk $>$ cotton, but the ratios of the adsorption constants varied with different dyes.

${ }^{x}$ Cf. Bayliss, op. cit., p. 6o, for instances of specific adsorption; also Bancroft's Applied Colloid Chemistry, p. 3.

${ }^{2}$ Freundlich, Kapillarchemie, p. I55. 
Wöhler and Plüddemann ${ }^{1}$ found that iron oxide adsorbed ro times as much benzoic acid as acetic acid; chromic oxide adsorbed both about equally; while platinum sponge adsorbed more acetic than benzoic, but both slightly. According to Freundlich, gelatine adsorbs sugar only after having been treated with formaldehyde. ${ }^{2}$ The influence of the specific molecular structure of the adsorbent on its selective adsorption is well shown in the investigations of Marc. A crystalline adsorbent, $\mathrm{BaCO}_{3}$ (rhombic) adsorbs $\mathrm{KNO}_{3}$ (rhombic) but not, or slightly, $\mathrm{NaNO}_{3}$ (hexagonal); $\mathrm{CaCO}_{3}$ (hexagonal) adsorbs $\mathrm{NaNO}_{3}$ but not $\mathrm{KNO}_{3}{ }^{3}$ These observations throw an interesting light on the phenomena of crystallization; it is well known that the specific molecular configuration of a substance determines the form in which it crystallizes, as originally shown by Pasteur's observations on the separation of levo- and dextrotartrate in separate crystals in the crystallization of the optically inactive solution. Apparently the abstraction of molecules from solution and their deposition to form the regular solid structure or crystal are determined by conditions of the same kind as those determining selective adsorption. Adsorption of molecules at the surface of the crystal is a preliminary to the growth of the latter; this growth is evidently dependent on mutual apposition of molecules similar in configuration and dimensions and with their axes parallel. ${ }^{4}$ In organic growth-another

I Wöhler and Plüddemann, Z. physik. Chem., LXII (190\$), 664.

${ }^{2}$ Freundlich, op. cit., p. 514 .

3 Marc, Z. physik. Chem., LXXXI (19r3), 64 r.

${ }^{4}$ Crystal growth, in fact, appears to afford the clearest cases of specificity in adsorption. 
type of specific growth and form-determination-similar processes are almost certainly concerned.

The reversible character of many adsorption processes is a property of great biological importance and apparently one essential to certain physiological effects, such as narcosis, which are universal in living protoplasm. There is no doubt that this reversibility also plays an essential part in the normal chemical processes of protoplasm. The displacement of one adsorbed compound by another of greater surface-activity presupposes reversible adsorption, and various biological instances of this effect are known. Thus an adsorbed enzyme can be removed from an adsorbing surface by adding a more surfaceactive substance; e.g., rennin adsorbed by charcoal and added to milk will not coagulate the latter, but on the addition of saponin the rennin is set free and causes coagulation. A solution of rennin is inactivated by shaking with air, but not if saponin is present; the latter protects the enzyme by preventing adsorption at the air-water interface. ${ }^{\mathrm{x}}$

Similar cases of inactivation by shaking are cited by Meltzer and Shaklee. ${ }^{2}$ Apparently only the adsorbed enzyme is inactivated; it has already been mentioned that changes of physical state frequently result from adsorption; Ramsden's observation that proteins can be coagulated by shaking with air is an instance of the same phenomenon. ${ }^{3}$ Hence the prevention of adsorption through the presence of another surface-active com-

${ }^{x}$ Cf. the observations of Jahnson-Blom (I9r2), and SchmidtNeilson (Igro), cited in Bayliss' Principles of General Physiology, p. 70.

2 Meltzer and Shaklee, Amer. Jour. Physiology, XXV (I909), 8r.

3 Ramsden, Z. physik. Chem., XLVII (1904), 343. 
pound may be a factor in preventing physical and chemical alteration in living protoplasm. Preventive effects of this kind probably form a chief factor in the anrsthetic action of surface-active compounds, as well as in the protective action which they often exhibit (against salt action, hæmolysis, or mechanical injury). ${ }^{x}$

ADSORPTION AND DEPENDENT PHENOMENA IN COLLOIDAL SYSTEMS

In general, colloids of the suspensoid group are less readily adsorbed than those of the emulsoid group, in correspondence with the fact that the latter are usually: surface-active, the former not. For the same reason the suspensoids do not usually act as emulsifying agents, while many emulsoids are highly effective in this regard. The distinction, however, is not absolute, since fincly divided insoluble substances of various kinds may emulsify oils under certain conditions; ${ }^{2}$ what is essential is that the material should collect and form a continuous layer at the surface between the phases.

As a rule proteins are surface-active; their solutions have lower surface-tensions than pure water and they are readily adsorbed. The conditions are, however, complex; the degree of adsorption may vary with the same protein according to its state of subdivision (which varies with the salt content) or according to the $H$-ion concentration of the solution; the latter condition determines the proximity to the isoelectric point and hence

ऽ See pp. $207 \mathrm{ff}$.

${ }^{2}$ Cf. Bancroft, loc. cit.; Journal of Plyysical Chemistry, XVI (1912), 475 . 
the electrical properties of the particles, a factor which influences their adsorption. ${ }^{\mathrm{T}}$

Observations on the relation between the concentration of proteins in solution and the degree of their adsorption give many abnormalities, probably referable chiefly to variations in the aggregation state. ${ }^{2}$ The particles cohere and form larger aggregates which condense at surfaces, forming films of modified protein. Such processes are largely irreversible, and chemical change probably also enters as a factor; the changes in the properties of enzymes, dyes, and other colloidal compounds in adsorption are probably to be thus explained. Many cases of abnormal and irreversible adsorption belong here; such abnormalities are especially characteristic of colloids of the emulsoid group. The surface-activity of proteins, and the readiness with which they form films, filaments, and other coherent structures, at the surfaces where they are adsorbed, are undoubtedly properties of great biological importance; and we may assume that in the deposition of proteins in a solid or semi-solid state to form the more permanent structural elements of protoplasm such processes play a chief part. As already indicated, it seems probable that specific adsorption, a process apparently based on the tendency of molecules of similar configuration to cohere or coalesce to form larger aggregates, is the fundamental factor underlying the specificity of growth processes.

As already pointed out, adsorption may furnish the conditions for many of the chemical reactions in cells;

×Cf. Christiansen, cited by Pauli, Colloid Chemistry of Proteins, Philadelphia (1922), p. 89.

${ }^{2}$ Cf. Höber, op. cit., pp. 2 I 7 ff. 
i.e., material concentrated or condensed at the protoplasmic surfaces may in this manner first become capable of chemical interaction. In a recent paper ${ }^{1}$ Bayliss gives instances showing that in many reactions in polyphasic systems adsorption is the initial process which forms a necessary preliminary to the true chemical combination following.

We may conclude that the determination and control of chemical reactions by adsorption are universal in living protoplasm. The presence of colloidal complexes or "adsorption compounds"-e.g., compounds of lecithin with proteins, such as lecithin-ritellin (in the ethereal extract of egg yolk) and jecorin (dextrose and lecithin plus protein)-is frequent in organisms. Inorganic salts and ions are probably also largely present in a condition of adsorption; ${ }^{2}$ and the indications are that the action of the surface-active pharmacological compounds (especially the anæsthetics) is largely so determined. According to Loewe, the chief relation between lipoids and narcotic compounds is one of adsorption, ${ }^{3}$ although the relative solubilities of these compounds in the different protoplasmic phases (partition-coefficients) probably also enter as an important factor in narcotic action. ${ }^{4}$

The promotion of chemical action by adsorption is often called "adsorption-catalysis." Well-known examples are the formation of $\mathrm{H}_{2} \mathrm{SO}_{4}$ from $\mathrm{SO}_{2}$ in the presence of platinum, the reduction of various compounds

${ }^{x}$ Proceedings of the Royal Socicly, B, LXxxiv (1911), Sr.

${ }^{2}$ Cf. Pauli, loc. cit.

${ }^{3}$ Loewe, Biochem. Z., LVII (19r3), r6r; cf. pp. 200 If.

${ }_{4}^{4}$ See chap. ix for the relation of adsorption to narcosis. 
by hydrogen in the presence of platinum, and the oxidation of compounds by blood-charcoal (oxalic acid, etc.). The combination of tannin with leather is a good illustration of the determination of a chemical reaction by a previous adsorption; the tannin is first adsorbed, then it combines. The same condition is shown in the union of dyes with heat-denatured egg-white; the process is at first readily reversible (by acid), but not later, indicating that the first stage of the process is a close contact or adhesion, which is then followed by chemical combination. ${ }^{x}$ The toxin-antitoxin reactions and the opsonin reaction with leucocytes are further biological instances of a similar kind. According to Morgenroth, tetanus toxin is taken up or attached by living cells at $8^{\circ}$, but does not become active until $20^{\circ}$. In the action of enzymes adsorption processes play an important part, as already indicated. ${ }^{2}$

\section{INFLUENCE OF ELECTRICAL STATE OF SURFACE ON ADSORPTION}

The relations of electrostatic attraction or repulsion between the charged surface of the adsorbent and the charge on the particles of the dissolved substance constitute a factor of decisive importance in many adsorption processes. For example, acid dyes (whose colloidal particles are negatively charged) are as a class more readily adsorbed by suspended alumina (with positive particles) than by kaolin, a silicate with negative particles, and vice versa. Color bases show the reverse behavior, being adsorbed by substances which form

I Unpublished observations of my own.

${ }^{2} \mathrm{Cf}$. the data and discussion in Bayliss' textbook, p. 324. 
negatively charged surfaces; e.g., silicates, carbon, and adsorbents of chemically acid character, but not by hydrates like alumina or other positive adsorbents. ${ }^{2}$ Suspensoids of heat-denatured albumin show the same behavior; when the particles are marle positive by the addition of a little acid they become adsorbents for acid dyes, which also cause precipitation; while in the negative condition, i.e., on the alkaline side of the isioelectric point, they are precipitated by (and adsorb) basic but not acid dyes. The mutual precipitation of oppositely charged colloidal solutions when mixed in suitable proportions is an example of the same phenomenon.

The importance of the electrical factor in the general behavior of colloids is indicated by the remarkable changes in adsorptive and chemical properties which a given protein exhibits when the $\mathrm{H}$-ion concentration of the solution passes from one side of the isoelectric point to the other. On the acid side precipitation is induced by the anions of an added electrolyte, on the alkaline side by the cations, as Hardy first showed in the case of heat-modified egg-albumin. ${ }^{2}$ Recently the relation between the charged condition of the protein aggregates and their chemical and other behavior has been investigated in much detail by Loeb, ${ }^{3}$ who has determined many striking correlations between the physical and the chemical properties of proteins at varying $H$-ion con-

${ }^{2}$ Cf. Michaelis, Physikalische Chemic und Mcdisin, cdited by Korany i and Richter, Leipzig, II (1908), $34 \mathrm{r}$.

${ }^{2}$ W. B. Hardy, Procedings of the Royal Socicly, L.XII (1S00), 810.

3 For a summary of these important investigations (f. Loeb's recent book Proteins and the Theory of Colloidal Belutier, New Lork, 1922. 
centrations. As Paulir also has pointed out, many properties (viscosity, osmotic pressure, refractive index, precipitability by alcohol) pass through a minimum which is coincident with the isoelectric point.

In all colloids the electrical factor plays a special part in changes of aggregation state or dispersion. In precipitation by electrolytes adsorption processes enter; one or the other ion is adsorbed predominantly, i.e., the ion of opposite sign to the colloidal particle; accordingly this ion is the precipitant. In general the more readily adsorbed an electrolyte is, the more effective it is as a precipitating agent. Freundlich has shown this clearly for the salts of a number of organic bases. ${ }^{2}$ The relative precipitating effectiveness of the several salts, with colloidal arsenious sulphide, is shown in the following table:

\section{Salt}

Aniline chloride..........

P-chloraniline chloride......

Strychnine nitrate..........

Morphine chloride..........

Neufuchsin.............

\section{Precipitating} Concentration

4. I

2.2

0.39

0.36

0.30

The order of precipitating concentrations is the reverse of the order of relative adsorption.

Theoretically the two ions of an electrolyte should have different adsorption constants in relation to an adsorbing surface. The nature and quantity of the ions adsorbed will influence the electrical conditions at the interface, and, secondarily, all processes in which these conditions are a factor, such as colloidal stability,

${ }^{3}$ Cf. W. Pauli, loc. cit.

${ }^{2}$ Kolloid-Z., I (I907), 328; Kapillarchemie, p. 35 I. 
state of dispersion, cataphoresis and electrical endosmose, and catalytic action. The action of electrolytes on colloids shows many indications of adsorption effects; those ions which other evidence indicates are in general the most strongly adsorbed ( $\mathrm{H}, \mathrm{OH}$, polyvalent cations) have a correspondingly marked influence on the colloidal state. The influence of adsorption is shown with especial distinctness in the action of salts on protein solutions, as Pauli's work has especially shown; the characteristic curves relating temperatures of heatcoagulation, melting points of gels, and precipitability by alcohol to concentration of salt (when the salt is present in excess of that required to form stoichiometric compounds) are clearly of the adsorption type. ${ }^{x}$ The same is true for the influence of salts on the osmotic pressure of protein solutions. ${ }^{2}$

Apparently, in all processes where surface effects are concerned, the different salts of the same metal differ in their action according to the nature of their anions; according to Röntgen and Schneider ${ }^{3}$ the order of relative adsorption of anions is $\mathrm{SO}_{4}<\mathrm{Cl}<\mathrm{Br}$ and $\mathrm{NO}_{3}<\mathrm{I}$; this series corresponds to the characteristic lyotropic series of Hofmeister, which is shown not only in the above-cited work on proteins and in many of the physiological effects produced by salts but also in various purely physical phenomena involving surface factors

${ }^{x}$ Cf. Pauli's book (loc. cit.) for references.

${ }^{2}$ The data in my paper (American Journal of Physiology, XX (1007), I27) show a relatively great depressant action on osmotic pressure for low concentrations of salts and a curve corresponding to the adsorption type.

3 Röntgen and Schneider, Amn. Physik., XXIX (1SS6), 165. 
(influence of salts on surface tension, solubility, viscosity, catalytic action, etc.). ${ }^{x}$

\section{ADSORPTION OF IONS}

The difference between the adsorbability of the two ions of an electrolyte implies an influence on the potential difference at the phase-boundary; the surface receives the charge of the more adsorbable ion and the adjacent layer of solution the opposite charge. Potentials arising in this way have been called "adsorption potentials" by Freundlich, ${ }^{2}$ and they undoubtedly play an important part in colloidal phenomena, since the alteration of the surface charge is a chief factor in the changes of aggregation-state and other phenomena characteristic of colloids. Adsorption potentials may also be of great importance in influencing the character of the chemical changes occurring under the catalytic influence of surfaces.

There are many indications that the ions of water, $\mathrm{OH}$ and $\mathrm{H}$, are among the most readily adsorbed ions. This property is of special biological importance, since most forms of protoplasm are highly sensitive to variations in the concentration of these ions; and in certain cases this sensitivity has become a regulatory factor of the utmost delicacy. Free organic acids and bases are

I For a summary of the physical effects of salts showing the lyotropic series cf. Höber, op. cit., p. 3 Io.

${ }^{2}$ According to Freundlich the adsorption potential is not the potential between the interior of the solid phase and the adjoining solution, but that between an adhering immobile layer of solution and the mobile layer adjoining. Cf. Kapillarchemie, p. 243; also Report on the Physics and Chemistry of Colloids by the Faraday Society and the Physical Society of London (I92I), p. I46. 
as a rule more readily adsorbed than their salts. Mamy organic acids (the lower members of the fatty series) are highly effective in lowering surface-tension, while their salts have little influence; this effect, however, may be in large part attributable to the undissociated molecules. As a class, acids have a marked effect on the surface charges of indifferent solid substances, tending to make these surfaces positive; similarly bases make them negative. Both effects appear in very low concentrations (Perrin) ${ }^{\mathrm{I}}$ and are undoubtedly due to $\mathrm{H}$ and $\mathrm{OH}$ ions, respectively. The fact that adsorbent surfaces of the most widely varying chemical composition (carbon, hydrocarbons, silicates, oxides, metals) are thus affected indicates that adsorption rather than chemical combination in stoichiometric proportions lies at the basis of the effect. A slight change in $\mathrm{H}$ and $\mathrm{OH}$ concentration may thus have a very marked effect upon the potential difference across a surface; this is well shown in the curves given by Haber and Kiemensiewicz, ${ }^{2}$ and in the results of Perrin. ${ }^{3}$ The great effectiveness of the $\mathrm{H}$-ion as a precipitant for suspensions of indifferent substances probably depends on its high adsorbability as well as on its high velocity and special chemical properties.

At a certain concentration where $\mathrm{OH}$ and $\mathrm{H}$-iuns are adsorbed in certain proportions the surfaces will be electriçally neutral; this condition defines the isoelectric

${ }^{x}$ Cf. Perrin, Jour. Chim. Phys., II (1904), 6or.

${ }^{2}$ Haber and Klemensiewicz, Z. physik. Chem., L.XYII (1900), $3^{4} 5$.

${ }^{3}$ See especially Perrin's curve for naphethalene, reproduced in Freundlich's Kapillarchemic, p. 236. Fillis also describes this elica in the cataphoresis of oil droplets, Z. pleysik. (hem., CXXVIII (1911), 321 
point. The $\mathrm{H}$-ion concentration corresponding to this neutral position varies according to the special chemical character of the material and its adsorbent properties.

Various chemical effects apparently depending on unequal adsorption of ions are described in Freundlich's book; frequently these appear to be consequences of the displacement of one ion from an adsorbent surface by another which is more readily adsorbed. For example, many basic dyes (crystal violet or basic fuchsin) are chlorides of organic color-bases; when these dyes are adsorbed by charcoal, a large part of the combined chloride goes into solution as inorganic chloride. This result is explained by Freundlich as due to the high adsorbability of the color cation (dye- $\mathrm{HCl}=$ dye- $\mathrm{H}^{+}$ and $\mathrm{Cl}^{-}$) which displaces from the adsorbent the adsorbed cation; according to this view the chemical splitting is due to the unequal adsorbability of the two ions. The phenomenon may, however, be regarded as a consequence of the high adsorbability of the free base, which is present in the solution in consequence of partial hydrolysis; this base is removed, leaving the chloride in solution. ${ }^{2}$ It is known that many organic free bases are more highly surface-active (adsorbed) than the salts; thus Traube points out that the surface-tension of solutions of hydrochlorides of the alkaloids, cocain, atropin, and quinine is lowered by adding a little alkali, an effect due to the liberation of the free base; ${ }^{3}$ the latter will tend to be adsorbed and the hydrolysis will be promoted. The separation of

I Freundlich, op. cit., p. I68.

${ }^{2}$ Cf. Michaelis, Arch. ges. Physiol., XCVII (I903), 634.

3 Traube, Kolloidchem. Beihefte, III (IgI2), 237; cf. also Höber, op. cit., p. 215 . 
dyes on adsorbing surfaces in an insoluble form and the "denaturation" of proteins on surfaces are apparently phenomena of the same general kind; adsorption will promote hydrolysis or other chemical alteration in a compound if any reaction-product is more readily adsorbed than the original compound. From this point of view the general kinetics of adsorption-cataly'sis appear in a clearer light.

The heavy metal ions and the ions of trivalent metals appear to be adsorbed with especial readiness in many cases, and the solutions of their salts have a correspondingly great influence on the surface charge of colloidal particles and of porous partitions. In the case of suspended oil drops, Ellis ${ }^{\mathrm{I}}$ found the following concentrations of three chlorides to be equally effective in removing the charge; i.e., in rendering the particles electrically neutral.

$\begin{array}{llc} & \text { Mols. per Litre } & \begin{array}{c}\text { Relative } \\ \text { Concentration }\end{array} \\ \mathrm{AlCl}_{3} \ldots \ldots & 0.00026 & \mathrm{I} \\ \mathrm{CuCl}_{2} \ldots \ldots & 0.0089 & \mathrm{ca} .40 \\ \mathrm{NaCl} \ldots \ldots & 0.40 & \mathrm{ca} . \mathrm{I} 600\end{array}$

The relative actions of $\mathrm{Na}, \mathrm{Cu}$, and $\mathrm{Al}$ are approximately as $\mathrm{I}$ to 40 to $\mathrm{I}, 600$, indicating a rapid increase of action with increase of valence. The same rule is found in the precipitation of suspensoid colloids by electrolytes (rule of Schulze and Hardy), and indicates

${ }^{x}$ Ridsdale Ellis, Z. physik. Chem., LxxviII (19г2), 32r. Loeb's recent study of the effects of ions in altering the charge on suspended collodion particles gives a similar result (J.Gen. Pliysiol., V [1922], 109). In this case the collodion particles undergo precipitation when the P.D. against the medium falls below i6 millivolts. I3acteria are agglutinated at 15 millivolts according to Northrop and de Kruif (loc. cit.). 
that a relatively great adsorption of polyvalent ions is a general rule. The relative effectiveness of polyvalent cations in certain characteristic physiological effects, e.g., ion-antagonism, is of a corresponding order, indicating that in such cases the ions act by adsorption at the structural surfaces of the living system. Thus $\mathrm{Al}$ and $\mathrm{Cr}$ ions greatly prolong the activity of cilia in isotonic $\mathrm{NaCl}$ solution when present in concentrations of less than $\mathrm{M} /$ I00,000. ${ }^{\mathrm{I}}$

It should be pointed out that even if the adsorption constants of mono-, di-, and trivalent ions were equal, the trivalent ions should be effective in less than a third of the concentration of the monovalent ions, because, on account of the characteristic form of the adsorption curve, the ratio between the quantity adsorbed and the quantity remaining in solution is much higher in dilute than in concentrated solution; hence sufficient trivalent ions to neutralize the surface charge may be adsorbed from extremely dilute solution. This is Freundlich's explanation of Schulze's rule. ${ }^{2}$ Whetham's explanation, based on chances, ${ }^{3}$ is probably insufficient, although purely mathematical considerations would indicate that the statistical conditions to which he calls attention must play a part in the total effect. The ratio between the effective concentrations of $\mathrm{Al}$ and $\mathrm{Na}$ in the above-cited experiments seems, however, too great to be accounted for on this ground alone, and a high degree of adsorption of polyvalent cations must apparently be assumed.

I R. S. Lillie, American Journal of Physiology, X (I904), 430.

${ }^{2}$ Freundlich, Z. physik. Chem., LXXIII (19Iо), 385.

3 Whetham, Theory of Solution, p. 396. 
The importance of adsorption-potentials is shown most clearly in the phenomena of electrical endosmose, which are of great physiological importance and will be considered briefly below. The marked influence which slight quantities of acid and alkali have in altering the phase-boundary potentials, especially in the neighborhood of the isoelectric point, is a fact of special biological interest. Near this point the effect of variations in the $\mathrm{H}$-ion concentration upon the phase-boundary potentials is at its maximum; farther from the isoelectric point the difference caused by a given change in the $\mathrm{H}$-ion concentration is relatively slight. ${ }^{\mathrm{I}}$

The reactions of the tissue fluids in higher animals are slightly on the alkaline side of neutrality, and the reaction of the living protoplasm (because of the higher tension of $\mathrm{CO}_{2}$ within the cell) is presumably somewhat less alkaline and is probably not far from the isoclectric point of some of the structural proteins. Hence slight variations of the $\mathrm{H}$-ion concentration within the cell should have a correspondingly great effect upon the boundary-potentials of the corresponding cell-structures. Variations in the $\mathrm{H}$-ion concentration of the cell-medium would affect first of all the boundary-potential of the cell as a whole; i.e., that existing across the plasma membrane, and this is probably the chief reason why living tissues are frequently so sensitive to changes in the external $\mathrm{H}$-ion concentration. As already pointed out. this sensitivity has in some cases become the controlling factor in regulatory processes of vital importance to the whole organism, as in the respiratory nerve cells of vertebrates, which show an accelerated rhythm in ${ }^{s}$ Cf. Haber and klemensiewicz, loc, cit. 
response to an extremely slight rise of acidity due to increase in the $\mathrm{CO}_{2}$ of the blood.

\section{ELECTRICAL ENDOSMOSE}

Certain physical phenomena of great biological interest, having similar relations to the charged character of the interphasic surfaces, are those classed as electrical endosmose. The essential phenomenon is the transfer of fluids with or against an electric current traversing a porous partition immersed in an electrolyte solution. The fundamental conditions of this transport are the same as those determining the electrical convection of colloidal particles or emulsion droplets, except that in electrical endosmose the continuous fluid phase is the mobile one, the solid phase which forms the substance of the partition being fixed in position. Colloidal gels interposed in the path of a current exhibit this phenomenon, and it is well known in protoplasmic systems. ${ }^{\text {I }}$ There is no question but that effects of the same kind must occur normally in living matter, since the latter, during functional activity at least, is continually being traversed by the currents of the bioelectric circuits; and it seems probable that electrical endosmose plays a special rôle in the processes of secretion and adsorption, as suggested by various physiologists. ${ }^{2}$

IFor example, Hermann describes experiments showing the transport of water through tissues (muscle and nerves) in the direction of the positive stream when a constant current is passed through the tissue (Arch. ges. Physiol., LXVII [1897], 240).

${ }^{2}$ Engelmann, Arch. ges. Physiol., VI (1872), 97; Waymouth Reid, Phil. Trans., Series B, CXCII (I900), 239; Höber, Arch. ges. Physiol., CI (I904), 607; cf. also my recent paper in Biological Bulletin, XXXIII (I9I 7), r 35, I $70 \mathrm{ff}$. 
At present, however, our knowledge is insufficient for any final estimate of its physiological importance.

Since the effect depends upon the potential difference between the walls of the pores and the mobile fluid layer adjoining, all conditions that influence this potential difference affect the character of the movement. The rate of the movement or its direction or both may be thus affected. The effects of salts, acids, and alkalies are well illustrated in Perrin's investigations published in 1904. ${ }^{\mathrm{I}}$ The following table gives the results of a typical experiment. The diaphragm consisted of naphthalene, and varying solutions of $\mathrm{HCl}$ and $\mathrm{KOH}$ were used:

Solution

$\mathrm{n} / 50 \mathrm{HCl} \ldots \ldots \quad 2 \times_{10}-2$

$\mathrm{n} / \mathrm{100} \mathrm{HCl} \ldots . . \mathrm{10}^{-2}$

$\mathrm{n} / \mathrm{I} 000 \mathrm{HCl} \ldots \mathrm{IO}^{-3}$

$\mathrm{n} / 5000 \mathrm{HCl} \ldots . \quad 2 \times 10^{-4}$

$\mathrm{n} / 5000 \mathrm{KOH} \ldots 5 \times 10^{-11}$

$\mathrm{n} / \mathrm{I000} \mathrm{KOH} \ldots$ I0 $^{-11}$

$\mathrm{n} / 50 \mathrm{KOH} \ldots . .5 \times 10^{-13}$
$\mathrm{OH}$ conc. (n) $\begin{gathered}\text { Charge of } \\ \text { Diaphragm }\end{gathered}$

$\begin{array}{ll}5 \times 10^{-13} & + \\ \mathrm{IO}^{-12} & + \\ \mathrm{IO}^{-11} & + \\ 5 \times 10^{-11} & + \\ 2 \times 10^{-4} & - \\ \mathrm{IO}^{-3} & - \\ 2 \times 10^{-2} & -\end{array}$

Flow

$3^{S}$ to anode

39 to anode

28 to anode

3 to anode

29 to cathode

60 to cathode

60 to cathode

This experiment and others of a similar kind show that when a current of given intensity is passed through a partition, the rate and direction of transport are determined by the charge of the partition substance, and that this varies in a definite manner with the nature and concentration of the ions present.

Perrin also showed that the isoelectric point varied with the nature of the diaphragm; thus partitions of iodoform and glass were persistently negative, while those of $\mathrm{BaCO}_{3}$ and $\mathrm{CrCl}_{3}$ were positive. The electro-

'Perrin, J. Chim. Phys., II (1904), 601. 
positivity of any surface, whatever its chemical composition, was invariably increased by adding monovalent acid to the solution, and decreased by adding monovalent base. Polyvalent ions were very effective, reversing the sign of the partition-charge in extremely low concentrations; e.g., for a partition of chromic chloride these results were found.

\section{Solution}

Water slightly acid........

Same+.ooI $\mathrm{n} \mathrm{K}_{3} \mathrm{Fe}(\mathrm{CN})_{6} \ldots$

Same+.02 $\mathrm{n} \mathrm{K}_{3} \mathrm{Fe}(\mathrm{CN})_{6} \ldots$

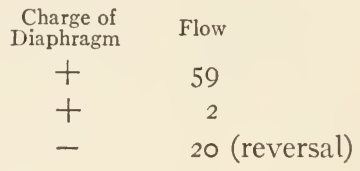

Freundlich ${ }^{\mathrm{r}}$ calculated from Perrin's data the concentration of salts (millimols per litre) required to reduce by 50 per cent the endosmose through positive and negative partitions, respectively, and obtained the following results.

I. $\mathrm{CrCl}_{3}$ (positive) Solution

Dil. acid $+\mathrm{KBr} \ldots \ldots \ldots \ldots \ldots$

$\mathrm{Same}+\mathrm{MgSO}_{4} \ldots \ldots \ldots \ldots \ldots$

$\mathrm{Same}+\mathrm{K}_{3} \mathrm{Fe}(\mathrm{CN})_{6} \ldots \ldots \ldots \ldots$

II. Carborundum (negative)

\section{Solution}

Dil. alkali $+\mathrm{NaBr} \ldots \ldots \ldots \ldots$

Same $+\mathrm{Ba}\left(\mathrm{NO}_{3}\right)_{2} \ldots \ldots \ldots \ldots$

Same $+\mathrm{La}\left(\mathrm{NO}_{3}\right)_{3} \ldots \ldots \ldots \ldots$.
Conc. (millimols per litre) for I/ 2 velocity 60 I O. I

Conc. for $\mathrm{I} / 2$ velocity

The effect thus increases very rapidly, in accordance with Schulze's rule, with increase in the valence of the active ion, which is always that having the opposite sign to that of the partition. Ellissafoff ${ }^{2}$ in an investiga-

${ }^{5}$ Kapillarchemie, p. 238.

${ }^{2}$ Ellissafoff, Z. physik. Chem., LXXIX (I9r2), 385. 
tion with glass and quartz capillaries also found the Perrin-Schulze valence rule to hold for alkali and alkali earth salts, but heary metals ( $\mathrm{Hg}, \mathrm{Ag}$ ) were more active than corresponded to their valence; organic cations (morphine, neufuchsin) were also highly active. Reversals with $\mathrm{Th}\left(\mathrm{NO}_{3}\right)_{4}$ and methyl violet were observed, but not with acids up to n/ rooo; no higher concentrations were used. ${ }^{\mathrm{x}}$

${ }^{x} \mathrm{~A}$ general review by T. B. Briggs in the Journal of Plyysical Chemistry for 1917 (XXI, 198, and XXII, 256) gives a full review of the subject of electrical endosmose. Recently its bearing on physiological phenomena and its relation to cases of anomalous osmosis have been considered in an important series of papers by J. Loeb (J. Gen. I'hysiol., II [1920], p. 557, and later papers in the same journal). For the action of ions see further, A. Gyemant, "Elektrocndosmose und Ionenadsorption," Kolloid-Z., XXVIII (1921), 103. 


\section{CHAPTER VI}

PROTOPLASMIC STRUCTURE-Continued: PERMEABILITY AND OTHER PROPERTIES OF PROTOPLASMIC MEMBRANES

We have seen that the stability of emulsion systems and many of their essential properties are determined by the presence of thin interfacial films. In the formation of these films, and in the determination of their special properties, electrical and other factors enter, of a kind characteristic of boundary surfaces in general. In general, it may be said that heterogeneous mixtures containing substances that influence the surface-tension or the electrical polarization at the phase-boundaries tend to reach a state of equilibrium in which they have what may be called a "structure"; i.e., a more or less orderly and definite distribution of the components. The gathering of surface-active compounds at the phase-boundaries, in conformity with the principle of Gibbs and J. J. Thomson, is a chief factor determining the character of this distribution; and the latter secondarily determines the character of the chemical changes occurring in the system. Living protoplasm is an example of a heterogeneous system in which the control of chemical change by structural conditions has reached perhaps its highest development.

Not only are the structural elements of protoplasm (alveoli, nuclei, colloidal particles, fibrils) bounded by surfaces at which adsorption and chemical change occur, but the whole mass of protoplasm, the living 
cell, is similarly bounded. Suspensions of living cellssuspensions of blood corpuscles, eggs, spermatozoa, bacteria, or yeast-in their nomal aqueous media may be regarded as similar in many respects to emulsions. Each cell is a small discrete particle with a surfacetension and an electrical potential-difference against its medium. The existence of this potential is shown in convection experiments; just as oil droplets migrate to the anode in an electrical field, so do suspended living cells; each cell, although living and highly differentiated internally, behaves in this respect like a negatively charged colloidal particle. As in the case of a suspended oil droplet, the sign of the charge carried by the living particle may be changed by acids or polyvalent ions; and like colloidal particles in general the cells may be precipitated from suspension (or agglutinated) under various conditions. ${ }^{\text {? }}$

Red blood corpuscles travel in neutral media like isotonic sugar solution to the anode, thus showing the presence of a negative surface charge; by passing $\mathrm{CO}_{2}$ through the media or adding weak acids, the sign of the charge may be reversed; the corpuscles then become positive or cathodic. ${ }^{2}$ At an intermediate concentration the charge is abolished (isoelectric point); and it is interesting to note that at this point the corpuscle tends to break down or undergo hrmolysis. ${ }^{3}$ This fact is of

I For data and references in this field, cf. Höber's Ploysik. Chemic d. Zelle, pp. 247, 300, 483, and 599. More recently the cataphoresis of bacteria and their agglutination by electrolytes and other substances has been studied by Northrop and De Kruif (J. Gcn. Physiol., IV |1922\}, 629,639 , and 655 ).

${ }^{2}$ Höber, Arch. ges. Physiol., CI (r904), 627; CII, 196.

${ }^{3}$ Michaelis and Takahashi, Biochem. Zcitschrift, XXIX (1910), 430. The isoelectric point is also the optimum for agglutination; cf. C. R. Coulter, Jour. Gen. Physiol., III (1920), 309 . 
special interest as indicating the importance of electrical factors in the structural stability of the protoplasmic surface layer, and suggests a reason why depolarizing influences have in general a stimulating action on irritable cells. The relations between structural change and stimulation will be considered in more detail later.

Höber and Kozawa ${ }^{x}$ found this isoelectric point to vary for different species of corpuscles and to be characteristic for a particular species; i.e., certain corpuscles are more readily made positive than others by $\mathrm{H}$ ions and polyvalent ions; thus the corpuscles of rabbits and guinea pigs were found to require the least $\mathrm{H}$-ion concentration for reversal and those of the ox and pig the highest; those of the dog, cat, goat, and man were intermediate.

Animal

Isoelectric Point (PH)

Rabbit. . . Between 3.8 and 3.4

Guinea pig..............ca. $3 \cdot 4$

Man (and cat)........... ca. 3.13

Dog.................. Between 3.13 and 2.98

Sheep ............... Between 2.98 and 2.77

Pig.................. ca. 2.77

The relative order with La ions was the same. Such differences are to be referred to the specific peculiarities of structure, composition, or permeability characteristic of each kind of corpuscle.

In their general or common features, these phenomena indicate that the behavior of the protoplasmic free surface with reference to the ions present in the medium resembles that of the surface of an individual oil-droplet in an emulsion. It may be assumed that

${ }^{2}$ Höber and Kozawa, Biochem. Zeitschrift, LX (I9I4), I46. 
the external surface of the cell is not peculiar in this respect, and that other protoplasmic interfaces (those within the cell) have similar properties. Film-formation, variations of electrical polarization, and dependent phenomena are thus to be regarded as constant features of the processes at such interfaces; e.g., at the surfaces of contractile fibrils, neuraxones, vacuoles, nuclei, alveoli, and other protoplasmic structures. Secondary effects peculiar to living protoplasm and dependent on metabolism may be superposed on these simple physical effects.

An important difference between the structure of protoplasm and that of a typical artificial emulsion, like oil in water, is that the two phases separated by a protoplasmic film are not necessarily aqueous and nonaqueous, respectively, but may both be aqueous. Any suspension of living cells, such as blood, illustrates this condition; both the interior of the corpuscle and the serum are complex aqueous solutions, and the film bounding the corpuscle is too thin to be optically detectable as a separate structure. Yet it can readily be shown by osmotic methods that a semi-permeable membrane is there present. Similarly within the limits of a single cell various structurally distinct regions, sometimes optically distinguishable, sometimes not, are to be regarded as separated by thin films. The details of this film structure will naturally vary in different types of cell.

The surface layer of the entire cell, the plasma membrane, is the modified surface-film which separates the internal protoplasm from its surrounding medium and through which the necessary interchange of material 
is effected. During life it behaves as if it were semipermeable and water-insoluble; yet it is evident that it allows passage, at least at certain times, to water and many dissolved substances; e.g., food-substances. Because of its special character as an intermediary structure or organ, its properties require special consideration in any study of the properties of protoplasm.

In some respects the properties of the plasma membrane appear difficult to explain on known physical grounds or even paradoxical. If a diffusible foodsubstance passes through the membrane into the cell it may there undergo metabolism, but it is difficult to see how any such substance can enter without other important substances leaving. If simple diffusion alone is the chief factor in the entrance and exit of dissolved materials, we should expect that any increase of permeability sufficient to allow the entrance of substances like sugar would also involve a loss of diffusible cell constituents to the exterior. It seems necessary to assume that the conditions determining the normal transport of substances across the cell boundary are of a special kind, and that diffusion is only one of a number of factors. In fact we know that in secretion and absorption special physiological mechanisms of transport are concerned, by which water and dissolved substances are actively conveyed into and out of the cell, frequently against concentration-gradients. This process requires the performance of work by the cell, just as does the process of muscular contraction, although its exact conditions are at present unknown. Many substances (sugar, salts, amino-acids, urea) may. thus be transported from regions of lower to regions of higher 
concentration, a process necessarily involving osmotic work; in this work $\mathrm{O}_{2}$ is consumed and energy set free. The problem of the nature of this physiological transporting mechanism is often distinguished as the problem of "physiological permeability" from the simpler problem of the conditions of simple diffusion across the cell surface, or "physical permeability." The majority of recent studies on protoplasmic permeability have had reference to this latter problem.

It will be apparent that the problem of the nature and conditions of cell-permeability is not a special or limited one but underlies the whole problem of the essential nature of protoplasmic structure and activities. Apparently the most fundamental property of the plasma membrane is semi-permeability; water can pass, although with considerable resistance in many cases; but the normal water-soluble constituents of the protoplasm and its surroundings do not diffuse across the cell boundary, or only under special conditions. This property of "semi-permeability" is associated with a high degree of electrical resistance (signifying impermeability to crystalloidal ions) and water-insolubility. The physicochemical conditions of these properties of the plasma membrane have been much investigated of recent years, and since the results of this work have an important bearing on our general problem, a somewhat detailed review will be given.

The general importance of membranes in organic structure and activities has long been recognized, and much study has been devoted by physiologists to various artificial types of membrane, such as precipitation membranes, membranes of parchment and collodion, 
and various impregnated types of membrane, in the hope of throwing light upon the peculiarities of living membranes. The resemblance of these artificial structures with the protoplasmic membranes seems, however, in many cases remote. The conditions observable in living protoplasm indicate that membranes of a different and somewhat special type, viz., the interfacial films formed between the separate phases in emulsions and other polyphasic systems, have a closer affinity with protoplasmic membranes than any other simple type of physical structure.

The property of forming thin films at structural surfaces of all kinds is highly characteristic of living protoplasm. The entire cell body is separated from the surrounding medium by the plasma membrane; within the cell the nuclear area is sharply delimited by a membrane which usually disappears only during mitosis; structurally distinct membranes are also formed about fibrils, vacuoles, alveoli, chromatophores, spheres, chromosomes, and various cell-inclusions. Apparently the conditions required for the formation of thin solid films are present everywhere in living protoplasm; at any structurally well-defined surface, continuous sheets of material may be deposited; the formation of membranes at cut surfaces and about extruded portions of protoplasm is simply one illustration of this general property. ${ }^{\mathrm{x}}$ The fertilized eggs of marine animals (sea-urchin, starfish) are especially favorable objects for showing this property; new films are rapidly formed

${ }^{\mathrm{I}}$ For a recent account of these phenomena, see the review by Seifriz, Annals of Botany, LXXXVIII (1921), 269; cf. also Botanical Gazette, LXX (1920), 360. 
at the surfaces of cuts made with microdissection needles, or around isolated portions of protoplasm. ${ }^{\mathrm{I}}$

Apparently the nearest inorganic analogies to these protoplasmic film-structures are the thin surface-films deposited at the boundaries between mutually insoluble liquids, one or other of which contains surface-active (especially colloidal) materials in solution; good illustrations are the films of soap or other material surrounding the droplets in an oil-water or other emulsion, or the solid films formed about globules of oil, mercury, chloroform, or other insoluble liquids immersed in proteincontaining solutions, or the haptogen membranes formed at the surfaces of warm soap solution, milk, or solutions of protein, peptone, saponin or other surface-active colloidal substances. Under certain conditions these films may acquire a solid consistency and exhibit considerable structural density, and thus limit diffusion between the two phases; the resulting system may then be described as triphasic, the three phases being the internal medium, the external medium, and the intervening thin phase or membrane. The "artificial cells" described by Harvey, ${ }^{2}$ made by breaking up a chloroform solution of lecithin in dilute egg-albumin, may be cited as examples of structures formed under these conditions.

In living protoplasm it is to be presumed that condensation-films of protein and other surface-active substances are formed at all of the surfaces bounding

I Chambers, American Journal of Physiology, XLIII (I9I7), I; Proceedings of the Society of Experimental Biology and Medicine, XVII (г919), 41; Jour. Gen. Physiol., V (I922), I89. For plant cells cf. Prowazek, Biol. Zentr., XXVII (I907), 737. Drops of protoplasm from Vaucheria form membranes about their surfaces.

${ }^{2}$ E. N. Harvey, Science, XXXVI (I912), 564. 
the different phases; and the physical resemblances between protoplasm and emulsions are apparently referable to the presence of these surface-films. Both systems are examples of what may be called filmpervaded or film-partitioned systems. By the formation of these films a certain structure is imparted to the whole system; this structure is largely the expression of surface-forces, in which chemical, electrical, and mechanical (surface-tension) factors all enter.

It should be noted that according to this conception of protoplasmic structure no essential distinction is to be drawn between the intracellular surface-films or membranes (alveolar membranes, vacuole membranes, nuclear membranes) and the surface-films inclosing entire cells (plasma membranes). In plant cells it can be shown experimentally that vacuole membranes and plasma membranes are simitar in osmotic properties, and Hamburger has shown the same for the nuclear membranes and plasma membranes of animal cells. ${ }^{\text {I }}$

In forming a general conception of the physicochemical characteristics of protoplasmic membranes, the properties of colloidal gels, especially in their relation to diffusion-processes, may be taken as a starting-point. In a gel, i.e., a solid mixture of colloidal material (such as gelatine) and water, diffusion is hindered only slightly if the concentration of the colloid is low. When the gel is made denser-as the proportion of water is decreaseddiffusion becomes slower and more restricted. At a sufficiently high density certain solutes, especially colloids, can no longer diffuse through the gel, although water and crystalloids may still pass. If the density

I Hamburger, Osmotischer Druck u. Ionenlehre, III, $8 \mathrm{ff}$. 
be still further increased, crystalloids and finally even water fail to pass. ${ }^{I} \quad$ This last condition is exemplified in water-proof organic membranes like the external skin of most animals and the membranes of certain eggs (the Fundulus egg). From an elementary or purely physical point of view a membrane may be regarded as essentially a thin sheet consisting of a gel of the kind above. Such a gel has a large surface-area in proportion to its total volume, and by virtue of its diffusion-hindering property it prevents or retards the transfer of material (colloidal particles, molecules, ions) between the two solutions which it separates.

Connected with this diffusion-hindering property, which conditions the rate of interchange and hence the rate of chemical activity at the surface between the solutions separated, are certain electrical properties ("membrane-potentials"), resulting from the influence of the membrane on the distribution and transfer of ions between the two solutions. ${ }^{2}$ These properties are apparently of fundamental importance to the bioelectric processes, and their conditions will be considered more fully later.

Independently of its structural density, a membrane of complex chemical composition may exhibit a selective

I The conditions may be compared with those presented by a series of "ultra-filters" of graded densities, as described by Bechhold (Colloids in Biology and Medicine); the permeability decreases as the density increases.

${ }^{2}$ Cf. Lewis, A System of Physical Chemistry, II (I920), 320, for an account of membrane potentials. The type of equilibrium investigated by Donnan, in which solutions are separated by a membrane which is impermeable to some but not all of the ions, plays an important part in many membrane processes, as shown especially by Loeb in his recent work. Cf. Proteins and the Theory of Colloidal Behavior, Part 2. 
permeability with reference to substances which are unequally soluble in its different chemical components. Substances soluble in a given component (lipoid-soluble substances) may thus pass a protoplasmic membrane, while chemically similar but lipoid-insoluble substances may not. Overton's experiments on the differences between the rates of diffusion of various organic compounds and salts into living cells illustrate selective permeability of this type. ${ }^{\mathrm{I}}$

It is important to recognize that the plasma membrane is not a dead structure or a purely passive partition, but represents in reality a portion of the living protoplasm, characteristically modified in its structure and physical properties by surface conditions. ${ }^{2}$ Hence it is the seat of metabolic and other activities which influence its physical properties. Evidently it is that part of the cell which comes into the most direct relations with the surroundings. Hence many changes in the surroundings influence the cell primarily through their action on the plasma membrane, and there is evidence that in irritable cells this structure plays the part of a specially sensitive and reactive intermediary between the living protoplasm and the external world; it thus exerts a far-reaching control over the metabolic and other processes occurring in the cell-interior. The relations of the plasma membrane to stimulation will be considered in detail later.

${ }^{x}$ Cf. Overton's summary of his work in Nagel's Handbuch der Physiologie, II (1907), 744.

${ }^{2}$ Cf. my paper in American Journal of Physiology, XLV (I9I8), 406, for a more complete discussion of this phase of the problem of permeability. 
The normal semi-permeability of the plasma membrane is a function of the living state of the cell, i.e., is dependent upon the continuance of the normal constructive metabolism. When metabolism ceases, as at death, the membrane soon loses its insulating or semi-permeable properties, and free interchange of diffusible substances then occurs between the cell and the medium. The loss of turgor in plant cells after death is the most familiar example of this type of effect; leaves and other parenchymatous parts then wilt because of the diffusion of the osmotically active substances through the now permeable plasma membranes. The osmotic tension which during life keeps the cell walls in their normal stretched and rigid condition disappears, the tissue becomes soft and flaccid, and the protoplasm shows other evidences of increased permeability (increased electrical conductivity, loss of diffusible materials to the surroundings, ready entrance of dyes and other substances). Similarly in animal cells various substances, such as pigments and other compounds, normally confined within the protoplasm, diffuse rapidly into the surrounding medium on death, and the plasma membrane admits substances such as alkalis and salts, to which previously it was impermeable.

It is a remarkable and apparently paradoxical fact that the living plasma membranes usually show themselves highly impermeable to many dissolved substances which are essential to the cell as foods or otherwise (sugars, amino-acids, and neutral salts), and some general explanation of this peculiarity seems required. If we were to express the matter teleologically we might say that the advantage to the cell consists in the insulation of the living protoplasm from its environment. 
which has an entirely different composition. The maintenance of the normal vital properties requires that the essential diffusible constituents of protoplasm should not be lost to the surroundings; it is also evident that too ready an entrance of substances from the outside would interfere with the stability of protoplasmic composition. The presence of a semi-permeable boundary layer appears thus to be a necessary condition for the preservation of the normal chemical organization of the cell. It is readily seen, for example, that the existence of a simple diffusion equilibrium between surrounding medium and protoplasm would prevent the latter from acquiring the special crystalloidal content which is characteristic of it. Hence, living cells are enabled to survive and develop largely by virtue of being inclosed by surface-films which are impermeable to crystalloidal compounds of the foregoing classes.

But since these compounds do in fact gain entrance to the cell, at least at certain times, it is clear that the problem of cell-permeability is not a simple one. Apparently we must conclude that the entrance or exit of substances by simple diffusion is in most cases a different phenomenon from their entrance or exit under physiological conditions. The processes of absorption and secretion are in fact special activities, requiring the performance of work by the cell. The distinction between a passive or purely physical permeability and an active or physiological permeability thus seems a necessary one.

The conditions of passive permeability are of interest chiefly because of the light which they throw upon the physical and chemical nature of the substances composing 
the plasma membranes. The most significant general fact is that apparently all substances with solubilities or solvent properties characteristic of organic compounds (rather than of water-soluble compounds or water) enter cells with special readiness. A relation between the presence of organic solvents in protoplasm and the permeability of the plasma membrane is thus indicated. Overton, who first investigated in detail this connection between organic solubility and power of penetration, drew the conclusion that the plasma membrane consisted essentially of the so-called "lipoid" compounds, especially lecithin and cholesterol, which are universally present in protoplasm. He showed that all members of homologous series, such as alcohols, ethers, esters, normal and substituted hydrocarbons, ketones, aldehydes, amides, and similar compounds, readily enter living cells; such compounds dissolve, or are dissolved by, the lipoids or solutions of lipoids in organic solvents; and their ready entrance is a result of this solubility. On the other hand, sugars and polyatomic alcohols (pentites, hexites, etc.), with molecules containing many hydroxyl groups, are highly soluble in water, but not in lipoids, and do not enter cells readily. In Overton's original experiments, acid and basic dyes also showed a relation between lipoid-solubility and power of penetration; but the conditions are complex in this case and many exceptions to this rule are now known. ${ }^{\mathrm{I}}$ In the case of neutral salts of alkali and alkali earth metals (especially $\mathrm{Na}$, $\mathrm{K}$, and $\mathrm{Ca}$ ) there is also little or no evidence of penetra-

${ }^{\circledR}$ Cf. Ruhland, Jahrb. wiss. Botanik, XLVI (rgos), I; cf. also Höber's discussion, Physikalische Chemie der Zelle und der Gewebe, pp. 426 ff. 
tion in balanced solutions, a fact corresponding to the lipoid-insolubility of these compounds. In pure solutions (pure $\mathrm{NaCl}$ solution) the salts alter the properties of the plasma membranes and secondarily may penetrate; but this fact is in no way inconsistent with Overton's view, which applies to the unaltered membrane. A definite correlation between lipoid-solubility and power of penetrating the living plasma membrane may be said to have been established by Overton's work and succeeding studies of the same kind; and this generalization is an important one, since it indicates (as do many other facts) that the lipoids play an essential part, apparently in association with the other chief colloidal compounds of protoplasm, the proteins, in the formation of membranes and probably of the other solid structural elements of cells.

Three chief methods have been employed in determining the permeability of cells to dissolved substances: (I) the plasmolytic or osmotic method, ${ }^{2}(2)$ the partition method, ${ }^{3}$ and (3) the electrical conductivity method. ${ }^{4}$ To these may be added methods dependent on the use of indicators, either those normally present in cells, ${ }^{5}$

${ }^{I}$ Cf. Osterhout, Science, XXXIV (Igri), I87.

2 Overton, Arch. ges. Physiol., XCII (I902), II5.

3 Hedin, Arch. ges. Physiol., LXVIII (I897), 229.

${ }^{4}$ Tangl and Bugarszky, Zentr. Physiol., XI (1897), 297; G. N. Stewart, ibid., p. 332; Osterhout, Scicnce, XXXV (I9I2), II2, and later papers; also Osterhout's recent book, Injury, Recovery and Death in Relation to Conductivity and Permeability, Philadelphia, I922.

5 Harvey, Internat. Z. physik.-chem. Biol., I (1914), 463; Crozier, Journal of Biological Chemistry, XXIV (1916), 255, and Jour. Gen. Physiol., IV (1922), 723; Haas, Journal of Biological Chemistry, XXVII (rgI6), 225. Cf. also Jacobs, American Journal of Physiology, LIII (I920), 457 . 
or dyes like neutral red $^{\mathrm{I}}$ which may be introduced from outside. These methods are especially valuable in studying the permeability to acids or alkalis. Permeability to dyes may usually be studied by direct observation.

The method of plasmolysis, first employed systematically by Overton, is based on the production of osmotic effects (entrance or exit of water) when the cell is placed in solutions having a different osmotic pressure from that of the cell-contents (anisotonic solutions). In hypertonic solutions of substances which do not readily traverse the plasma membrane the cell shrinks, in hypotonic solutions it swells, while in isotonic solutions its volume remains unchanged. The problem is to determine the relative degree of permeability to different substances, some of which may traverse the membrane, but with unequal readiness. To do this the behavior of the cell is observed in a hypertonic solution of the substance under examination. It is clear that when the dissolved substance in the external medium is quite unable to penetrate the membrane, it exerts pressure against the latter (by the continued impacts of the molecules), and since this pressure is greater than that exerted by the dissolved molecules within the cell, water is extracted (or expressed) from the latter until a permanent state of equilibrium is reached in which the osmotic pressure of the cell-contents equals that of the surrounding solution. The cell is then permanently shrunken. When, however, the dissolved substance

${ }^{1}$ Bethe, Arch. ges. Physiol., CXXVII (1909), 219; Warburg, Z. physiol. Chem., LXVI (1910), 305; Harvey, Journal of Experimental Zoölogy, X (I9I I), 507; Chambers, Jour. Gen. Physiol., V (1922), I\$9. 
penetrates gradually, its water-abstracting effect is only temporary, since its concentration, at first greater outside than inside the cell, is eventually equalized by diffusion; the cell then tends to resume its original water-content. In the case of a solute which penetrates rapidly (with a readiness like that of water) no effective inwardly directed pressure can be exerted against the membrane and no osmotic effect is produced.

Using plant cells (Spirogyra and others) and moderately hypertonic solutions of various substances, Overton found that in solutions of sugars, amino-acids, and neutral salts the plasmolysis was permanent; in solutions of glycerine, glycol, urea, and similar compounds the degree of plasmolysis was less than in sugar solutions, and after the initial shrinkage, water gradually reentered the cell; while in solutions of alcohols and many other organic substances (of the same osmotic pressure as the effective sugar solutions) plasmolysis was entirely absent. Similar differences were typical of a large number of other compounds. He therefore divided soluble substances into three groups, according to their ability to penetrate the living plasma membrane: (I) Those to which the plasma membrane is completely or nearly impermeable, including sugars, polyatomic alcohols (from erythrite up), soluble amino-acids, neutral salts of alkali and alkali earth metals; (2) those which penetrate the membrane, but slowly and with varying degrees of resistance, including glycol, glycerol, and certain amides such as urea; and (3) those which enter without encountering any evident resistance; here belong a variety of organic compounds of the groups cited above. These general conditions were found by 
Overton and other investigators to be characteristic both of animal cells (blood corpuscles, muscle cells, egg cells, etc.) and plant cells of the most varied kinds. In the partition method, as used by Hedin and others, the distribution of dissolved substances between the cells and the solution is measured directly (by cryoscopic determinations), and its results agree closely with those of the plasmolytic method. In general it has been found that the above-cited conditions of permeability are highly characteristic if not universal in living protoplasm. ${ }^{x}$

Since in general the lipoid-soluble substances which penetrate living protoplasm are also highly surfaceactive, the conclusion may be drawn that either lipoidsolubility or surface-activity (or both) is a property favorable to penetration (Overton, Traube). The penetration of one substance through another may depend on mutual solubility; the cases of the rubber membranes used in Flusin's experiments, the watersoaked bladder partitions employed by Nernst, and the palladium partitions of Ramsay's experiments with nitrogen and hydrogen may be cited as illustrations. ${ }^{2}$ Overton explains the permeability of the plasma membrane to lipoid-soluble substances as an expression of the solubility of these substances in the lipoids of the membrane; in general he finds a parallelism between the lipoid-water partition-ratio of a given substance and its ability to penetrate cells; this is illustrated by the behavior of substitution-products and of the members of

${ }^{x}$ Cf. Hamburger's Osmotischer Druck u. Ionenlehre for an account of comparative investigations in this field.

${ }^{2}$ Flusin, Ann. de chim. el phys., XIII (1908), 480; Nernst, Z. physik. Chem., VI (I89o), 37; Ramsay, Z. physik. Chem., XV (1894), 518. Cf. p. 144 below. 
homologous series. The contrast between $\mathrm{NH}_{4} \mathrm{OH}$ (lipoid-soluble) and $\mathrm{KOH}$ (lipoid-insoluble) in their ability to enter living cells is a striking one; ${ }^{\mathbf{I}}$ and most investigators who have studied the penetration of substances (like acids and bases) whose behavior can be determined with accuracy, have found a general parallelism between the rate of penetration and the lipoidsolubility of the various compounds. This parallelism, however, is not exact, indicating the presence of other factors. ${ }^{2}$

Impermeability to neutral salts in balanced solution is an especially important property of the plasma membrane, since it renders possible permanent differences of salt-content between protoplasm and surroundings. This means differences of ionic content, and is probably a condition of the normal electrical polarization ("physiological polarization") of the membrane, since any partition separating two solutions of different ion-content is typically the seat of an electrical potential difference. The results of the mineral analysis of cells, as well as those of the plasmolytic and partition methods just described, show clearly the inability of neutral salts to

${ }^{\top}$ Cf. Warburg, loc. cit.; Harvey, loc. cit.; Gray, Proceedings of the Royal Society, B, XCIII (1922), ro4 (cf. p. r1o); Jacobs, Jour. Gen. Physiol., V (r922), r8r. A similar contrast exists between organic acids (lipoid-soluble) and strong acids; cf. Loeb, Biochem. Zeitschrift, XV (r9o9), 254; Bethe, loc. cit.; Gray, loc. cit. Jacobs'results especially show the extraordinary ease with which carbon dioxide and ammonia penetrate living protoplasm.

${ }^{2}$ Harvey, Intern. Z. physik. chem. Biol., I (r9r4), 463; Crozier, loc. cit. Miss Collett's studies on the toxicity of acids to infusoria show a similar general relationship between organic solubility and toxicity (Journal Experimental Zoölogy, XXIX [r9rg], 443, and XXXIV [192r], $67,75)$. 
penetrate the unaltered living cell. Paine ${ }^{\mathrm{x}}$ has shown experimentally that $\mathrm{NaCl},\left(\mathrm{NH}_{4}\right)_{2} \mathrm{SO}_{4}$, and $\mathrm{Na}_{2} \mathrm{HPO}_{4}$ in $\mathrm{r} /$ Io solution do not enter yeast cells appreciably even after hours of immersion; and numerous analyses have shown that the specific salt-content of many living cells (blood corpuscles, muscle, etc.) is quantitatively or even qualitatively entirely different from that of the medium. For example, sodium salts seem to be almost completely absent from vertebrate muscle cells. ${ }^{2}$ This result seems incompatible with more than a very limited permeability of the plasma membrane to these substances. Either the salts do not diffuse across the membrane, or some active physiological factor is at work which opposes or compensates the effect of diffusion and maintains the salt content of the protoplasm at a certain norm. The nature and proportion of the salts present in any species of cell are characteristic of that cell and apparently represent a constant feature of its chemical organization; this is illustrated, for example, in the analyses of the salt-content of mammalian blood corpuscles by Abderhalden and others. ${ }^{3}$ In the corpuscles of the horse, pig, and rabbit there is little or no $\mathrm{Na}$ and an abundance of $\mathrm{K}$; in the ox, sheep, goat, dog, cat, the amount of $\mathrm{Na}$ is greater than that of $\mathrm{K}$. Inorganic phosphates are always much more concentrated in the corpuscles than in the serum, which is always rich in $\mathrm{Na}$ and poor in $\mathrm{K}$. Voluntary muscle cells are rich in $\mathrm{K}$ salts and phosphates and poor in $\mathrm{Na}$ salts.

I Paine, Proceedings of the Royal Socicty, B, LxXXIV (I9г2), 2S9.

${ }^{2}$ Urano and Fahr, cf. chap. viii.

3 Höber, loc. cit., p. 370. 
Höber (op. cit., p. 37I) cites certain observations of Warburg made in $\mathrm{I}_{\mathrm{I}} \mathrm{II}^{\mathrm{I}}$ indicating that the erythrocytes of the goose are influenced in their oxygen-consumption by alkali-earth salts only after the plasma membrane has been destroyed by freezing and thawing. Apparently these salts cannot pass the intact plasma membrane. On the other hand, alcohols and urethanes check oxidations in the intact erythrocytes; these substances can penetrate. It may be noted that these observations are consistent with the view that the nuclear surface is a chief factor in the oxidation-processes of these cells. ${ }^{2}$

The relative or complete impermeability of blood corpuscles to the ions of the surrounding salt solution is also indicated by the low electrical conductivity of these cells. Stewart, Tangl, Bugarszky, and others have shown that the electrical conductivity of blood is due almost entirely to the plasma. Low electrical conductivity is in fact now known to be a highly constant and characteristic peculiarity of cells during life, and the evidence indicates that the high resistance is chiefly if not entirely a property of the plasma membranes. The conductivity of the cell as a whole appears to vary directly with the permeability of the plasma membrane to crystalloidal solutions. All conditions that increase general permeability (action of cytolytic substances or unbalanced salt solutions or of poisons, high temperatures, or other lethal agents) also increase electrical conductivity. According to Osterhout, the most exact

I Warburg, Z. physiol. Chem., LXX (rgrr), 4r3.

${ }^{2}$ In the nucleated erythrocytes of the frog, the indophenol test shows active oxidation at the nuclear surface; cf. R. S. Lillie, Journal of Biological Chemistry, XV (1913), 237. 
measurements of permeability are those given by electrical conductivity. The conductivity of a living tissue is a measure of its permeability to ions; and while it is conceivable that the permeability to other substances, such as non-electrolytes like sugar, may vary independently (within certain limits) of the permeability to ions, the advantages of estimating permeability quantitatively are such that the conductivity method must be regarded as the one to be preferred wherever it can be applied.

Osterhout has shown that by means of the conductivity method the permeability of plant tissues under varying external conditions can be readily and accurately determined; also that permeability can be varied at will, reversibly, in either direction, especially under the influence of neutral salts and lipoid-solvent compounds. ${ }^{\mathrm{I}}$ In pure $\mathrm{NaCl}$ solutions the permeability of Laminaria fronds is increased, to a degree depending on the duration of exposure and the temperature; on replacing the tissue in sea water the permeability returns to or toward the normal, the degree of possible recovery depending upon the extent of the change produced by the $\mathrm{NaCl}$ solution. ${ }^{2}$ If the permeability has been increased beyond a certain limit, its reversal is impossible and the plant is dead. He has suggested, therefore, that the property of "vitality" may be measured by determining the electrical resistance. ${ }^{3}$ Isotonic $\mathrm{CaCl}_{2}$ solutions have the opposite kind of effect and at first decrease permeability; the antagonism between $\mathrm{Na}$ and

'Osterhout, loc. cit., and Science, XXXVII (Igr 2), 3; also "Quantitative Researches in Permeability," The Plant W'orld, XVI (I9I3), I 29.

${ }^{2}$ Osterhout, Jour. Gen. Physiol., III (1920), I45.

3 Osterhout, Science, XL (I9I4), 488. 
$\mathrm{Ca}$ salts is thus explained; when the two salts are present in appropriate proportions $\left(20 \mathrm{NaCl}+\mathrm{I} \mathrm{CaCl}_{2}\right)$ conductivity is unaltered, and the tissue remains living for days, while in the pure solution of either salt toxic action and death soon result. The changes of permeability accompanying normal physiological processes have also been measured by the conductivity method in certain cases, especially by McClendon and Gray in the fertilization of sea-urchin eggs. ${ }^{x}$

The question of the condition of the salts in the living protoplasm arises here; and this question is of considerable general importance, since it has been held by certain investigators that these salts are present chiefly or entirely in a combined or adsorbed state and are hence not free to act as conductors. As we shall see later in dealing with the phenomena of stimulation and transmission, the electrical conductivity of the internal protoplasm appears to be a necessary factor in its normal activity; and any evidence that this conductivity is what we should expect it to be from the known saltcontent of protoplasm is of interest. The contention that the salts in protoplasm are non-ionized, or that the ions are in some manner rendered immobile, is inconsistent with the physico-chemical observations relating to the behavior of salts in the presence of proteins, lipoids, or other colloids. According to Bugarszky and Liebermann, the addition of even large quantities of protein to salt solutions affects the ionic concentration only slightly; Michaelis and Rona ${ }^{3}$ have shown by "com-

${ }^{x}$ McClendon, American Journal of Physiology, XXVII (I9го), 240; J. Gray, Journal of the Marine Biological Association, X (1913), 50.

${ }^{2}$ Bugarszky and Liebermann, Arch. ges. Physiol., LXXII (I898), 5 I.

3 Michaelis and Rona, Biochem. Zeitschrift, XIV (Igo8), 476. 
pensation dialysis" that the salts in serum (containing Io per cent protein) are freely dialyzable. Pauli and Samec have also shown that alkali salts are not more soluble in serum than in water. ${ }^{\text {I }}$

There is the possibility of the formation of combinations with the amino-acids present in the proteins; but the salts of such weak acids would theoretically be almost completely hydrolyzed; i.e., a stable combination with protein in which the salt is completely and firmly bound is scarcely conceivable. Any protein-salt combinations thus formed would hydrolyze, and the products of hydrolysis would diffuse out through the membrane if the latter were permeable; and further hydrolysis would proceed until an equilibrium was reached in which a large proportion of salt was present in the free dissolved state. $^{2}$

General chemical theory thus indicates that only a very small proportion of the salt in the cell can be in a state of permanent combination with protein; hence the characteristic difference between the salt-content of the cells and that of the medium cannot be thus explained. It is probable, therefore, that the semi-permeability of the plasma membrane is an essential factor in making possible this difference.

If it were possible to measure the electrical conductivity of the cell interior apart from that of the plasma membrane, the question could be answered at once. According to the view presented above, the chief

'Pauli and Samec, Biochem. Zeitschrift, XVII (I909), 235.

${ }^{2}$ In other words, the proteins and other compounds present in the cell could not hold more than a very small proportion of the salts in a combined and indiffusible form. 
barrier to the movement of ions is at the semi-permeable plasma membrane. The increased conductivity observed at death, or after cytolysis by saponin or other compounds, favors this view, since semi-permeability is then lost; i.e., there is a general parallelism between the ability of salts, sugars, and other soluble compounds to pass the plasma membranes and the electrical conductivity

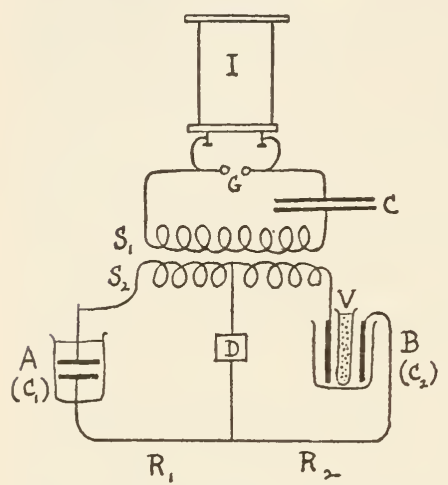

FIG. I.- $I$, inductorium; $C$, condenser; $G$, spark-gap; $S$, coil (self-induction) of primary circuit; $S_{2}$, coil of secondary circuit; $A$, adjustable condenser; $B$, condenser with space between plates for insertion of vessel $(V)$ containing suspension of cells; $D$, detector in bridges $R_{\mathrm{x}}, R_{\mathrm{z}}$, resistances (from Höber, loc. cit.). -a fact indicating that the chief condition rendering living cells such poor conductors of electricity is the impermeability of the membranes to ions. From these considerations we should conclude that the ions within the cell are free to diffuse within the space inclosed by the plasma membrane. But the case requires to be tested by experiment, for it might be held, in spite of the foregoing considerations, that the electrolytes are in some manner chemically combined in living protoplasm and are set free only at death.

Höber has attempted to measure the conductivity of the cell interior, using in part methods suggested to him by Nernst. A device of the plan shown in Fig. I was first employed. ${ }^{I}$ A rapidly alternating current is induced in the circuit containing the two balanced condensers

× Höber, Arch. ges. Physiol., CXXXIII (rgro), 237. 
$A$ and $B$. When the capacities of the condensers are the same and the resistances of the two halves of the circuit (on either side of the bridge) are the same (or when their ratios are equal; i.e., when $\left.\frac{R_{1}}{R_{2}}=\frac{C_{2}}{C_{1}}\right)$, current passes through the detector; but if the capacity of one condenser $(A)$ is changed, the current passes until the capacity of the other $(B)$ is made the same. Now the capacity of a condenser is increased if a conducting layer is introduced into the dielectric between the plates, and to a degree which is proportional to the conductivity of the layer. Höber's method, therefore, consists in introducing between the plates of one of the condensers $(B)$, after the system has been brought into a balanced condition, a glass vessel containing a suspension of living cells (blood corpuscles) in isotonic sugar solution. Such a suspension does not conduct electricity (in the usual Kohlrausch sense), yet it increases the capacity of the condenser and so allows a current to flow across the bridge. This result shows that the suspension contains an electrically conducting fluid; this can only be in the interior of the cell, since the suspension-medium is sugar solution, which is a non-conductor. The addition of saponin (which destroys the membrane and increases the Kohlrausch conductivity) was found not to change the capacity, indicating that the conductivity of the cell-contents is the same whether the semipermeable membrane is present or not. If a glass vesscl containing a salt solution is placed between the condenser plates, a similar increase of capacity is shown; and by comparing the effects produced by salt solutions of known conductivity with those produced by suspensions 
of cells, the internal conductivity of the latter can be estimated. The measurements cannot be made very exact; they indicate, however, that the internal conductivity of the corpuscle is of the order of that of a o.I $n$ $\mathrm{KCl}$ solution (between o. I $\mathrm{n}$ and o.or $\mathrm{n}$ ).

Höber also experimented with another method somewhat different in principle. ${ }^{x}$ If high-frequency oscillations are induced ( $\mathrm{IO}^{7}$ per second) in a circuit containing a condenser and a detector (spark-gap), and the beaker ( $B$, Fig. 2), encircled by a coil of wire forming part of

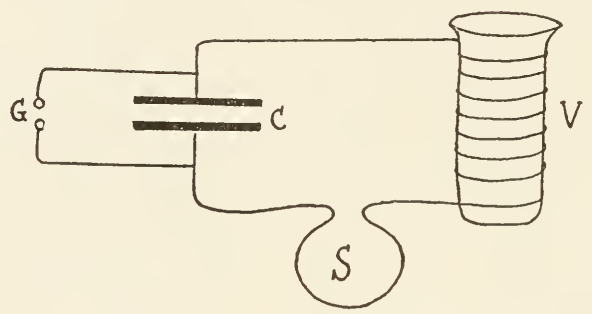

FIG. 2.- $C$, condenser; $V$, beaker containing suspension of corpuscles; $G$, sparkgap; $S$, coil in which current is induced (Höber, loc. cit.).

the circuit, is filled with a conducting solution, the oscillations are damped. Höber again found that cells suspended in sugar solution produce this effect, and to the same degree whether they are intact or cytolyzed with saponin, although the Kohlrausch conductivity is, of course, much greater in the latter case. The low Kohlrausch conductivity of intact cells is thus apparently due to the inclosing plasma membranes; the internal conductivity of the protoplasm is high. Again by comparing the effects produced by cellsuspensions with those produced by salt solutions of

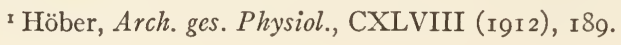


known conductivity, Höber found that the suspension gave the same effect as a $\mathrm{NaCl}$ solution of a concentration similar to that of blood plasma (i.e., between o.I and 0.4 per cent). Further accuracy is not possible.

Höber also used the method of measuring the conductivity of cells by means of rapidly oscillating currents. ${ }^{I}$ According to theory, we should expect that the higher the oscillatory frequency the less difference would the presence of the membrane make. Under the conditions, frog's muscle, which had been washed thoroughly in sugar solution, behaved as if it had a conductivity between 0.1 and $0.2 \mathrm{NaCl}$; the greater the frequency of alternation the greater the conductivity. This is readily understood if we reflect that when the carriers of the current (ions) have to pass through only very short distances, they are not impeded in their movements by the membrane.

From the results of the experiments above Höber reaches the following conclusion:2 "It can be regarded as certain that the blood corpuscles possess a very considerable internal conductivity, even when the external conductivity is minimal; i.e., that free ions are present in their interiors, and that these are prevented from reaching the outside only by the presence of a barrier to diffusion"; i.e., the plasma membrane.

The observations cited by $\mathrm{Kite}^{3}$ and others, as indicating the impermeability of the internal protoplasm to diffusing substances, are probably to be explained as indicating the rapidity with which a semi-permeable

${ }^{x}$ Höber, Arch. ges. Physiol., CL (1913), I5.

${ }^{2}$ Höber, Physikalische Chemie der Zelle und der Gewebe (1914), p. 3.5 .

${ }^{3}$ Kite, Biological Bulletin, XXV (1913), I. 
barrier is formed at any free surface of living protoplasm. ${ }^{\text {I }}$ This property of forming fresh semi-permeable surface films at exposed protoplasmic surfaces has long been known. Nägeli ${ }^{2}$ describes experiments with the root hairs of Hydrocharis; by crushing these structures under a cover-glass the protoplasm may be expressed in the form of separate rounded vacuolated masses. Each of these shows the same osmotic properties as the original entire protoplast; i.e., shrinks in hypertonic solutions, resists the entrance of dyes (during the living state), and in general behaves as if it were surrounded with a semi-permeable membrane. Such an experiment might also be regarded as indicating the impermeability of all parts of the protoplast to dissolved substances. But it can be simulated by the simple experiment of breaking or cutting up a large drop of an insoluble liquid, such as chloroform, in a protein-containing medium; each resulting droplet remains separate and exhibits the same properties as the original droplet, and the effect can be shown to depend on the rapid formation of a thin protein adsorption-film at the surface of each newly formed droplet. In a somewhat similar manner, although the conditions are more complex, living protoplasm forms films at surfaces which are freshly exposed by cutting or other injury; this property is shown only during life and is presumably a manifestation of the normal property of construction and repair, which is dependent on metabolic synthesis, as we have seen. This is indicated by an experiment of Pfeffer's, ${ }^{3}$ in which root hairs are placed in

${ }^{x}$ Cf. Chambers, Jour. Gen. Physiol., V (1922), 189.

${ }^{2}$ Nägeli, Pflanzenphysiologische Studien, Zürich (I885).

3 Osmotische Untersuchungen (1877), p. 136. 
a weak solution of acid, which kills the cells. If then they are placed in a weakly hypotonic solution containing dye, the latter immediately enters as soon as the cell swells; i.e., the continuity of the plasma membrane is then no longer automatically maintained (as in living cells) when the membrane is stretched or ruptured.

Presumably in living protoplasm both metabolic and purely physical factors (adsorption) take part in the formation of new surface-films. There is evidence that the protoplasmic surface-films undergo a change in their physical properties soon after they are formed; this is well shown in the freshly cut surfaces of sea-urchin eggs. Küster found that the protoplasm of plant cells could be broken into fragments by strong plasmolysis; these fragments at first readily unite or cohere, but later lose this property. ${ }^{\mathrm{I}}$ In the coalescence of fragments of inert inorganic material a similar behavior has been observed; freshly formed surfaces reunite readily, but not older surfaces; apparently the progressive deposition or adsorption of foreign materials at the surfaces alters their properties and prevents fusion. ${ }^{2}$ There is also evidence that the formation of new surface-films plays an essential part in the normal return of irritable cells to the resting state after stimulation; during the period of recovery of excitability (refractory period) the cell-surface is apparently the seat of progressive changes of this kind (see below under refractory period).

Höber cites the case of myxomycetes possessing a clear surface hyaloplasm and a granular interior; 3 when

${ }^{x}$ Küster, Ber. deutsch. botan. Ges., XXVII (1909), 589 .

${ }^{2}$ For an account of these phenomena cf. Bancroft's A pplicd Colloid Chemistry, chap. v, on coalescence.

3 Höber, op. cit. (rgI4), p. 64. 
the cell swells in hypotonic solution the thickness of the hyaloplasm remains constant; apparently this thickness is regulatively maintained by the transformation of material derived from the internal protoplasm. Chambers' observations on marine eggs show many interesting instances of the formation of films at cut surfaces or at the surfaces of protoplasmic fragments; regions where the protoplasm is broken down by mechanical or other injury soon become delimited by films bounding them from the adjoining unaltered protoplasm. ${ }^{\mathrm{I}}$ In the formation of artificial vacuoles by the injection of solutions into egg cells through micro-pipettes, films with semi-permeable properties are formed about the introduced droplets. ${ }^{2}$ The composition of the salt solution is an important factor in the formation of such vacuoles; Chambers has recently shown that pure solutions of $\mathrm{NaCl}$ diffuse into the protoplasm without forming films, while if sufficient $\mathrm{CaCl}_{2}$ is present, each droplet of solution surrounds itself with a definite film and forms a vacuole. A recent study by Seifriz ${ }^{3}$ of the physical properties of protoplasm, as exhibited under micro-dissection, gives many interesting details on filmformation by living protoplasm under various conditions.

De Vries showed in $1885^{4}$ that the normal vacuoles of plant cells are surrounded by membranes having the same osmotic properties as the plasma membranes (those inclosing the entire protoplast). In his experi-

${ }^{s}$ Chambers, loc. cit.

${ }^{2}$ Kite, loc. cit.; Chambers, Jour. Gen. Physiol., V (1922), I89.

3 Seifriz, loc. cit.

${ }_{4}$ Jahrb. wiss. Botanik, XVI (I885), 465 . 
ments the cell was placed in a ro per cent solution of $\mathrm{KNO}_{3}$ containing some eosin to serve as indicator of permeability; the outer protoplasm dies in one or two hours and becomes colored, but the vacuole remains at first clear and uncolored, showing impermeability of its membrane to the dye. Later the membrane becomes permeable to the dye, presumably as a result of deathchanges, and the vacuole contents become colored. Isolated vacuoles show osmotic properties similar to those of the whole protoplast. The observations of Kite and Chambers on artificial vacuoles in sea-urchin eggs illustrate the same phenomenon, the limiting surfacefilm of the vacuole having apparently the same properties as the surface-film of the entire cell.

The evidence just cited shows the error of regarding the properties of continuous protoplasm as identical with those of partitioned protoplasm. At boundary surfaces of whatever kind the living substance exhibits special properties, in particular a high resistance to the diffusion of water-soluble substances including ions. On the other hand, the internal protoplasm appears (at least in many cases) to be freely penetrable to diffusing substances of low molecular weight and to ions; hence it possesses a considerable electrical conductivity. This latter conclusion is of great importance for the theory of stimulation and conduction, as will be seen below.

As additional evidence for the permeability of continuous or unpartitioned protoplasm to water-soluble substances, we may cite the type of distribution shown by many diffusible substances in cells. Many such substances are concentrated in special regions or structures, in which they appear to be held in chemical or other 
combination; a good illustration is the water-soluble red pigment of Arbacia eggs, echinochrome, which is contained chiefly in minute round chromatophores scattered throughout the protoplasm. That this material is free to diffuse is shown, however, by the fact that cytolytic action, even temporary, causes its rapid diffusion into the surroundings; and complete loss of pigment may thus result. The localization or condensation of the pigment in the chromatophores indicates apparently that it is present there in some form of loose chemical combination or adsorption; but in any such case an equilibrium between the adsorbed and the dissolved compound must exist $\left(\mathrm{C}_{\mathrm{ads}}=\mathrm{KC}_{\mathrm{sol}}^{\mathrm{r} / \mathrm{n}}\right)$. Presumably the dissolved portion of the pigment is homogeneously distributed throughout the protoplasm, hence the chromatophores adsorb (or combine) equal quantities and are similar in appearance. But any local decrease in the concentration of dissolved pigment, due to diffusion through the altered plasma membrane, disturbs the adsorption equilibrium and leads to the liberation of the adsorbed pigment, which may thus be completely lost from the cell. The conditions are similar in any other case of reversible adsorption.

Hermann's observations on the difference between the transverse and the longitudinal conductivity of nerve fibers also indicate the higher conductivity of nonpartitioned protoplasm as compared with partitioned. $\mathrm{He}$ found that the current encounters several times the resistance when the fibers are placed transversely between the electrodes, as compared with fibers arranged lengthwise, with equal distances between the electrodes and equal sectional areas of tissue. The same is true for 
muscle. ${ }^{I}$ This result indicates again that the interposed plasma membranes offer a high resistance to the movement of ions, while the resistance of the continuous protoplasm is relatively slight. ${ }^{2}$

I Hermann, Arch. ges. Physiol., V (1872), 223. The character of the intercellular partitions and their arrangement will, of course, affect the inner conductivity of the cell, but regarding these conditions we have little information at present.

${ }^{2}$ Compare the conclusions of Stewart (J. Pharmacol. and Exper. Therapeutics, I [1909], 49); also Chambers (1922), loc. cit. 


\section{CHAPTER VII}

GENERAL CONDITIONS DETERMINING THE PROPERTIES OF PROTOPLASMIC MEMBRANES

Overton's and later researches have shown that the permeability of plasma membranes to lipoid-solvent substances is a universal property, that the permeability to water-soluble substances of low molecular weight, not soluble in organic solvents, is relatively very slight, and that the ability of substances to penetrate most forms of protoplasm has a close dependence on their relative solubility in the two classes of solvents; i.e., on their partition-ratios between organic (oil-like) solvents and water. The permeability for the two classes of substances, water-soluble and "organo-soluble," thus depends on different conditions. It is further significant that the permeability for lipoid-soluble substances is much less variable than that for the lipoid-insoluble substances and water; the latter form of permeability shows wide variations in the same cell under different physiological conditions, while the former appears to undergo little change. The characteristic semi-permeability of the living plasma membranes thus relates to the lipoidinsoluble group of substances; this fact, when considered in connection with the universal permeability to the lipoid-soluble group, suggests that the normal semi-permeability depends on the presence in the membranes of water-insoluble compounds possessing the solvent properties of organic solvents. The characteristic water-insolubility of the surface- 
layer of protoplasm is also intelligible on this point of view.

When we say that a cell or tissue is permeable to a given substance, we state merely the ability of the substance to penetrate; the method of penetration is not stated. Thus a dissolved substance may penetrate a partition by diffusing through the interspaces, or by dissolving in or combining chemically with the substance of the partition. In the case of ions there may be an apparent penetration resulting from a change in the electrical conditions at the surface of the partition. For example, when a current is passed by zinc electrodes through a bath of a zinc salt solution divided into two compartments by a zinc partition, it is not strictly correct to say that the zinc ions penetrate the partition. The effect is the same as if they did, but in reality zinc ions are deionized and deposited as zinc on one face, while from the other face the metal passes into solution as zinc ions. There is apparently a penetration in such a case, but not in reality; and it is possible that under certain conditions the passage of a current through a plasma membrane may similarly depend on the chemical combination of ions on the one face simultancously: with their release from the other. The physical and chemical conditions of these various types of permeability require careful examination.

The factors determining the penetration of dissolved substances through living plasma membranes are various. and for the purpose of the present discussion they may be grouped under the following heads: (I) structural conditions, including thickness, density (water-content). state of dispersion of structural colloids, size of interstices 
or pores, (2) chemical conditions, including special composition and solvent properties of constituents, (3) physical conditions of other kinds, such as electrical polarization at the faces of the partition or other surfaces, electrical endosmose effects, filtration effects, and (4) factors dependent on the living condition of the membrane, including variation of permeability due to metabolic or other changes; the last-named factors are apparently the ones chiefly responsible for the active types of material transport in and through cells in absorption and secretion (factors of "physiological permeability").

A complete discussion of these various factors need not be attempted here, especially since the whole subject of permeability has recently been carefully reviewed in the well-known textbooks of Bayliss and Höber. Certain aspects of the problem of permeability require special consideration, however, especially the dependence of permeability on physical conditions, and the nature of the factors controlling the normal or physiological variations of permeability. The most characteristic peculiarity of the living protoplasmic membranes, especially of irritable cells, is that their properties vary with both the external and the internal conditions, and are subject to regulative control of a highly definite kind. Such properties cannot be derived from any simple static type of structure, or explained on the basis of the peculiarities of the colloidal compounds composing the membrane. We must recognize that the distinctively vital factors, those depending on the metabolic activity of the cell, are probably the most important of all, and they are the least understood at present. The simpler 
physico-chemical or non-vital factors can, however, be shown in some cases to have definite relations to certain characteristic physiological effects; e.g., in the action of salts and lipoid-solvent compounds on living cells. These are the substances through whose action the physiological properties and activities of cells may most readily be altered in a reversible manner; and this action is referable in many cases to a direct alteration of the plasma membranes.

\section{PERMEABILITY AND STRUCTURAL CHARACTER OF MEMBRANES}

Semi-permeability requires a certain closeness of physical texture; i.e., density of structural material; apparently also the structure-forming substances must be water-insoluble. In all semi-permeable artificial membranes water-insolubility is essential; the most perfect examples are the precipitation-membranes of the ferrocyanides and other insoluble salts of heavy metals. That the chief compounds composing the plasma membranes are also water-insoluble is shown by the characteristic insolubility of living cells in their normal aqueous media.

The relation between structural density and permeability in artificial membranes is well shown in the "ultrafilters" devised by Bechhold for the separation of various colloids from their suspension-media. ${ }^{\mathrm{I}}$ These filters consist of disks of gelatine hardened in formalin. Bechhold found that the higher the colloid-content of these partitions, the more numerous were the colloidal sub-

${ }^{x}$ Bechhold, Colloids in Biology and Medicine, English translation, or third German edition, I920. 
stances which were held back in filtration experiments; for example, disks of Io per cent content prevented the passage of nearly all the colloids employed. From such facts we should expect that a still higher degree of impermeability (to crystalloids) would require a higher degree of structural density; i.e., lower water-content.

Experience with artificial semi-permeable membranes of copper ferrocyanide bears out this expectation; this is well seen in Morse's studies of these membranes, described in his book, Aqueous Solutions. ${ }^{\mathrm{I}}$ He found that for the formation of good semi-permeable membranes an extremely fine porosity in the supporting porcelain cells was necessary; "excessive fineness of texture is absolutely indispensable to the correct measurement of osmotic pressure" (p. I5). A good semi-permeable membrane is thus essentially a fine-textured structure of water-insoluble material; hence it resists the passage of water as well as of dissolved substances. Morse also considers that the colloidal character of the precipitate is an important factor in semi-permeability. Even under the best conditions a high degree of semi-permeability is difficult to obtain with precipitation-membranes, and this property is subject to change; it is affected by temperature (cf. pp. 85-86) and especially by electrolytes (cf. pp. 9I-92) which cause rapid deterioration in the membrane. $\mathrm{LiCl}$ was the least harmful of the electrolytes investigated (cf. p. 2 I4) and Morse gives determinations of the osmotic pressure of this salt.

Since fineness of texture is favorable to semi-permeability (i.e., to the production of osmotic effects), one might expect that any insoluble porous partition would ${ }^{r}$ Morse, Aqueous Solutions, Carnegie Institute Publications (I9r4). 
show osmotic action (semi-permeability) if only its pores were sufficiently minute. In fact, Thomas Graham observed many years ago (1854) that certain fine-grained porcelains had definite osmotic action. Recently the pore-diameters of osmotically acting porcelain disks have been investigated by Bigelow and Bartell, ${ }^{\mathrm{x}}$ and well-marked osmotic effects were found when the pores had a diameter of the order $0.2-0.35 \mu .^{2}$

In general we may conclude from such facts that in living semi-permeable membranes the degree of porosity is low; i.e., the interspaces between the water-insoluble colloidal particles forming the structural material are at least as small as in porcelain and probably smaller, since Bartell's membranes are not completely semipermeable. The physical conditions are probably closely comparable with those existing in well-supported precipitation-membranes of copper ferrocyanide, such as Morse employed. In plasma membranes, however, variations in the subdivision of the colloidal constituents may cause variations in the size of the interspaces, and hence in the permeability. The progressive deterioration to which artificial colloidal membranes are subject in the presence of electrolytes is apparently prevented in living membranes by compensatory factors dependent on metabolism; presumably any interruptions of continuity are at once automatically repaired by the formation or deposition of new structural material (see below under stimulation).

${ }^{x}$ Bigelow, Journal of the American Chomical Socicty, XXIX (1)0; . I675; Bartell, Journal of Physical Chemistry, XV (IgI 1), 050, and XVI (I9I 2), 3 I8.

${ }^{2}$ Bartell, loc. cit. (191 2). 
Such fine-textured membranes must resist the passage of water as well as of dissolved substance. In Morse's experiments many days were often required to reach osmotic equilibrium. There is also evidence that many living plasma membranes offer a high resistance to the passage of water: i.e., are relatively water-impermeable. This statement may seem surprising in view of the fact that the passage of water into and out of living cells in anisotonic solutions is usually rapid; but the membranes are extremely thin and the ratio of the surface to the inclosed volume is very large, so that relatively rapid entrance of water is quite consistent with a very low specific permeability to the liquid.

The loss of semi-permeability at death is associated with an increase of permeability to water as well as to dissolve substances. This is shown in some experiments of Bernstein who, with another problem in mind (the conditions under which water is held in cells), determined the relative rates of evaporation of water from living and dead tissues. ${ }^{x}$ Bernstein used the method (introduced by Liebig) of measuring the "force of imbibition" of membranes. A tube widened at one end (thistle tube) is fixed vertically with its narrow end dipping into mercury; the tube is completely filled with water and the upper expanded end is closed by the membrane under examination. As water evaporates through the membrane the mercury rises in the tube, showing the development of a pressure; the rate of evaporation through the membrane is thus indicated. Bernstein used fine tubes ending above in equally sized funnels which were closed (A) with living membrane and (B) with dead membrane

${ }^{\approx}$ Bernstein, Electrobiologie, Braunschweig (IgI2), pp. I67 ff. 
(e.g., killed by heat). Results of the following kind were obtained with frog skin:

(A) Living

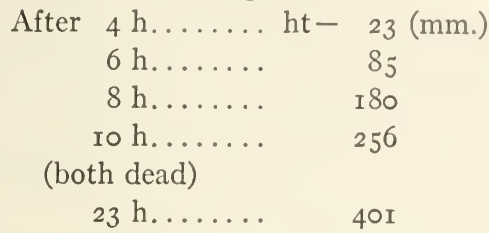

(B) Dead

$52(\mathrm{~mm}$.

I 60

285

347

400

It is clear that the rate of evaporation is much greater through the dead membrane. Similar experiments with plant tissues (leaves) gave similar results; von Mohl (in I847) and Naegeli (in I86I) had already called attention to the fact that frozen plant tissues (leaves, fruits) dried more rapidly than living. ${ }^{\mathrm{I}}$ Bernstein experimented also with thin sheets of muscles (abdominal oblique of frog), both living and killed with chloroform, and found that the rate of evaporation through the living muscle was about half of that through the dead; the difference was greatest during the first hour, when the living tissue was relatively normal.

Bernstein's conclusion was that living tissue has greater water-binding power than dead, and he regarded the electrically polarized condition of the plasma membranes as the chief factor hindering the outward passage of water. Apparently a potential difference of the value of $c a$. O.I volt exists normally between the outer and the inner surfaces of the plasma membrane; this gradient is positive externally and nega tive internally. Resistance is thus offered to the passage of the positively charged water outwardly across the membrane. This explana-

${ }^{x}$ Cf. Bernstein, op. cit., p. 170 . 
tion, while ingenious, does not seem sufficient; in muscle and other living cells immersed in anisotonic solutions water seems to pass with equal readiness in either direction through the membrane; for example, Arbacia eggs in hyper- and hypotonic sea water shrink and swell (respectively) at about the same rates. ${ }^{\mathrm{I}}$ Höber refers the difference observed by Bernstein to the greater turgor of the living cells; ${ }^{2}$ this explanation also seems doubtful, since turgor is either absent or slight in vertebrate tissues. The simplest as well as most probable explanation is that the permeability to water increases with death, along with the permeability to other substances. As the plasma membrane loses its semipermeability, with the associated fineness of texture, it also loses its relative impermeability to water.

Permeability to water is one of the little studied properties of cells. Yet it is an important property which appears to be constant for a particular cell under definite conditions. There is evidence that it varies with the physiological state and activity of the cell, and in certain cases, especially in gland cells, the indications are that it is under nervous control. Thus when the chorda tympani is stimulated, the submaxillary gland secretes a copious watery saliva; similarly the sweatglands and the kidney secrete actively under certain conditions, but not under others. In most cases the secretory substances leave the cell in aqueous solution; and although the factors are complex, there seems to be little doubt that the transport of the secretion across the cell-boundary is associated. with an increased permea-

${ }^{1}$ R. S. Lillie, American Journal of Physiology, XLV (1918), 406.

${ }^{2}$ Höber, op. cit., p. 255. 
bility to water as well as to dissolved substances. The character of the bioelectric variation accompanying secretion also indicates this.

Antropoff ${ }^{x}$ has studied the influence of the waterpermeability of osmometer membranes on the rate of osmotic transfer of water. He reaches the general formulation:

$$
\frac{d p}{d t}=\sigma\left(P-P_{t}\right)
$$

i.e., the rate of passage of water into an osmometer $\left(\frac{d p}{d t}\right)$ at any time is proportional to the permeability of the membrane to water $(\sigma)$ and to the osmotic pressure of the solution $(P)$ less the hydrostatic or other pressure $\left(P_{t}\right)$ resisting the transfer. The rate of osmotic transfer of water is thus proportional to the product of the effective osmotic pressure into the permeability to water. This formulation defines the conditions for perfect membranes; i.e., those which are permeable to water and impermeable to solute.

In the Arbacia egg the permeability to water is altered in a remarkable manner as a result of fertilization. A quantitative measure of this permeability may be obtained by measuring the rate at which water enters or leaves the egg in dilute or concentrated sea water. This rate is found to be increased about fourfold after fertilization, indicating that this process is associated with a marked increase in permeability to water. If mixed fertilized and unfertilized eggrs are placed in dilute or concentrated sea water, the former undergo change of volume much more rapidly than the latter, and

${ }^{\mathrm{I}}$ Antropoff, Z. physik. Chem., LXXVI (19II), 721. 
within a minute or less the two can easily be distinguished by their difference in size. The semi-permeability of the plasma membrane is not impaired, although the permeability to water is increased. The conditions of this phenomenon are probably complex, and determined by metabolic factors of unknown nature. The change of permeability is progressive and occupies a considerable time, some 20 minutes elapsing $\left(\right.$ at $20^{\circ}$ ) before it nears its final stage; it is arrested by anæsthetics (chloral hydrate, urethane, alcohols) and by cyanide in higher concentrations. ${ }^{\mathrm{I}}$

If the impermeability to water in this cell is due to the presence of water-insoluble substances (lipoids or cholesterol) in the surface-film, the change above would indicate that these are altered or removed in part; i.e., a change in the chemical composition of the surface layer is to be assumed. The nature of this change is unknown; but Lyon's observation that the iodine-combining power of the egg is decreased after fertilization ${ }^{2}$ may indicate a decrease in certain unsaturated compounds which would otherwise take up the iodine-possibly cholesterol or unsaturated lipins (lecithin). The permeability of fertilized eggs to water can be artificially modified in certain ways; it is decreased by anæsthetics (chloral, urethane, alcohols), ${ }^{3}$ a fact also indicating a dependence on the lipoid-content of the protoplasmic surface-film.

Chambers finds certain differences in the behavior of fertilized and unfertilized echinoderm eggs in micro-

${ }^{x}$ R. S. Lillie, American Journal of Physiology, XL (1916), 249; XLIII (I9I7), 43; XLV (IgI8), 406.

${ }^{2}$ Lyon and Shackell, Science, XXXII (I910), 249.

${ }^{3}$ R. S. Lillie, American Journal of Physiology, XLV (1918), 427. 
dissection; the surface of the unfertilized egg is less easily cut or torn with a needle, but exhibits less power of repair than that of the fertilized egg. This latter difference is probably to be correlated with the increased rate of metabolism following fertilization; oxygenconsumption, $\mathrm{CO}_{2}$-evolution and heat-production are then greater, ${ }^{2}$ also the susceptibility to $\mathrm{KCN}$ poisoning and other forms of chemical injury. Facts of this kind illustrate the close correlation existing between the physical state of the plasma membrane and the physiological properties and metabolic activity of the cell. Other examples of this correlation will be described later, especially in relation to stimulation-processes, which are intimately associated with changes in the membranes.

\section{PERMEABILITY AND SOLVENT PROPERTIES OF MEMBRANE CONSTITUENTS}

It was first pointed out by Nernst in I 890 that the solvent properties of the substances composing a membrane may determine the latter's permeability to dissolved substances, and hence the production of osmotic effects. Thus when benzol is separated from ether by a partition consisting of bladder membrane soaked in water (in effect a layer of water), the ether, because of its greater solubility in water, passes the partition more rapidly than the benzol, and a pressure is set up on the benzol side of the partition. ${ }^{3}$ The membrane is impermeable to

${ }^{2}$ Chambers, Proceedings of the Society of Experimental Biology and Medicine, XVII (1919), 4r.

${ }^{2}$ Cf. Shearer, Procedings of the Royal Socicty, B, XCIII (1921), 2I $3,4 \mathrm{ro}$.

3 Nernst, Z. physik. Chem., VI (1890), 37. 
benzol, which is almost insoluble in water, while ether is readily soluble (to the extent of about I 2 volumes per cent) and penetrates the partition. Experiments illustrating the same principle may be performed with gases; thus if a tube, containing air, closed below with a water-soaked membrane and connected above with a manometer, is placed in a vessel of ammonia or hydrochloric acid gas, the pressure rapidly rises in the tube because of the penetration of the water-soluble gas through the water-layer. Ramsay's well-known experiment with a tube filled with nitrogen and separated from a hydrogen atmosphere by a palladium partition (at $350^{\circ}$ to $400^{\circ}$ ) illustrates the same general phenomenon; hydrogen but not nitrogen penetrates the partition, hence the pressure rises within the tube. ${ }^{\mathrm{I}}$

In all of these cases the permeability of the partition depends on its consisting (in part) of some material which acts as a solvent to the penetrating substance. Nernst called attention to the possibility that similar conditions might exist in living cells; i.e., that the solubility of substances in constituents of the protoplasmic membranes might be the condition of their selective absorption by cells; and this form of explanation was later adopted by Overton who reached the conclusion, as a result of his studies on permeability already described, that the protoplasmic surface layer consisted chiefly of compounds with solvent properties similar to those of typical organic solvents.

Later, chiefly because of experiments indicating a parallelism between the penetration of various acid and basic dyes into living cells and the solubility of the same

× Ramsay, Z. physik. Chem., V (I894), 518. 
dyes in solutions of lecithin and cholesterol. ${ }^{\underline{x}}$ () verton referred the characteristic permeability of the plasma membrane specifically to the presence of lipoids. This evidence is in itself scarcely conclusive, especially since other factors are now known to be of importance in the penetration of dyes into cells, and many exceptions have been found to the rule that lipoid-solubility connotes ready penetration. The state of colloidal subdivision has been shown to be an important factor, ${ }^{2}$ and the negative electrification of the cell-surface must also play a part by favoring the adsorption and penetration of the positive particles of the basic dyes, which Overton found to penetrate most readily. But other evidence of various kinds supports Overton's conclusion in the main; 3 and there can be little doubt that the lipoids form essential constituents of plasma membranes, even although other compounds, especially proteins, may be of equal importance; e.g., as furnishing a structural support to the lipoids. The influence of lipoid-solvents on the permeability and activity of cells, the characteristic cytolytic action of these compounds, the influence of salt-solutions on oil-water emulsions, the peculiar relations of cholesterol to the mechanical properties of the plasma membranes, the properties of artificial lipoidimpregnated membranes, and many other facts all indicate the important rôle of lipoids in determining the properties of plasma membranes. It is not to be con-

s Overton, Arch. ges. Physiol., XCII (1902), II 5.

${ }^{2}$ Ruhland, loc. cit.

3 Especially the results with strong and weak acids and bases, above cited. Cf. the discussion in chap. viii, pp. 428 ff., of Höber's I'/hysikulische Chemie der Zelle. 
cluded that these membranes are simple continuous sheets of lipoid; the very fact that their properties are complex and vary from species to species is inconsistent with any such simple view, and the existence of specific cytolysins may indeed be taken as proof that proteins form an essential part of their composition. In fact, as already pointed out, the plasma membrane is not to be regarded as a simple passive layer of colloidal or other material, but rather as a special living structure with a characteristic metabolism of its own, and with both its physical and chemical properties modified in correspondence with its situation at the cell-boundary. All that can safely be maintained is that its properties are intimately dependent on the properties of its lipoid constituents, hence vary with changes in the physical and chemical state of the latter. It will be unnecessary to review in further detail the large body of experimental fact indicating the presence of lipoids in the surface-films of cells; this evidence is discussed at length with full references to the literature in Höber's and Bayliss' textbooks.

\section{PERMEABILITY AND PHYSICAL, ESPECIALLY ELECTRICAL, CONDITIONS}

Of late years electrical conditions have been shown to be of great importance in determining the permeability of artificial partitions (parchment, porcelain, and other substances), and from general principles it seems certain that such factors must play a corresponding part in the protoplasmic membranes. In artificial membranes two factors have been shown to be of importance: (I) the potential-difference between the solutions in contact 
with the opposite faces of the partition, and (2) the potential-difference between the solicl or colloidal material composing the partition and the fluid occupying its interstices or pores. ${ }^{\mathrm{I}}$ The influence of these electrical factors is shown with especial clearness in the phenomena of electrical endosmose, and in the related phenomenon of negative or anomalous osmosis. In both phenomena an electrified layer of fluid (occupying the pores of the partition, hence in contact with the surface of the structural material) is situated within the electrical field between the two surfaces of the partition; the fluid is accordingly transported across the partition by electrostatic attraction in one or the other direction, so long as the electric conditions remain unchanged. The work involved in the transport is electrical; in negative osmosis the electrical field is maintained by the unequal diffusion-rates of the ions of the solution bathing the partition; in electrical endosmose by the external current traversing the partition.

It will be evident that under these conditions the permeability of the partition to electrified material, whether water, colloidal particles, or ions, will be different in the two directions. Water, for example, will diffuse outward in an osmometer, i.e., from the more to the less concentrated solution, when the electrical difiusion ficli is oriented in such a way that the charged linyer of tluid in the pores is attracted outwardly with sufficient force to overcompensate the purely osmotic effect. This condition is met when a stronger solution of $\mathrm{HCl}$ is

' Girard, Compt.rend., CXLVI (1908), 927; CXLVIII (1000), 1047; J. de Physiol. et de Path. gén., XII (1910), 47I; J. de C'him. ch de l'hys. XVII (r910), 383 . 
sparatet from a weaker solution by a porcelain partition; Pthedolution on the more dilute side is then positive, because of the diffusion field; the solid substance of the partition is positive and the water in the pores is negative; accordingly the latter is drawn toward the more dilute solution. Anomalous osmosis of this kind has long been known; Graham (1854) observed, for example, that $\mathrm{K}_{2} \mathrm{SO}_{4}$ showed positive osmosis in alkaline solution and negative in acid when a bladder membrane was used; $\mathrm{NaCl}$, on the contrary, showed positive osmosis in acid solution and was indifferent in neutral solution. ${ }^{\mathrm{x}}$

These changes in the direction of transport are now recognized as depending on the influence of the ions on the charged condition of the structural surfaces. Many surfaces are rendered positive by $\mathrm{H}$ ions and polyvalent cations; the adjacent water-layer, being then negative, moves in a corresponding direction in the potentialgradient between the two surfaces of the membrane. Water may thus move in one or the other direction according to the electrolyte content. The influence of ions on the direction of transport is shown with especial clearness in the experiments of Perrin ${ }^{2}$ and others on electrical endosmose. The influence of ions on anomalous osmosis has recently been studied by Loeb, using gelatinepermeated membranes of collodion; the effect varies with the $\mathrm{P}_{\mathrm{в}}$ of the solution and becomes minimal at the isoelectric point of gelatine. Isoelectric membranes can,

${ }^{5}$ Graham, Phil. Trans., CXLIV (1854), 117 . For a recent account of negative osmosis cf. Bartell, Journal of the American Chemical Society, XXXVI (19I4), 646; Bartell and Hocker, ibid., XXXVIII (1916), 1029, 1036; Bartell and Madison, Journal of Physical Chemistry, XXIV (1920), 444, 593.

${ }^{2}$ Perrin, loc. cit. 
however, become charged and exhibit anomalous osmosis under the influence of trivalent ions. ${ }^{\mathrm{x}}$

The transport of the ions of salts, and consequently the permeability of a given membrane to a diffusing salt, are similarly affected by the electrical state of the partition, and this influence has recently been studied by: Girard, using bladder membranes. If the polarization of the membrane (P.D. between its opposite faces) has a certain orientation, the penetration of a salt like $\mathrm{MgCl}_{2}$ or $\mathrm{Na}_{2} \mathrm{SO}_{4}$ in the one direction is facilitated, in the other direction hindered. ${ }^{2}$

The application of the principles above to the case of the plasma membrane is somewhat uncertain, since the structure of the latter is in many respects different from that of fixed porous membranes of macroscopic dimensions. Yet variations in the P.D. across the plasma membrane must have a corresponding effect on the permeability; this effect, however, is probably accompanied in living protoplasm by other effects, such as chemical effects depending on the electrode-like action of the membrane, and effects on colloidal dispersion. There is no doubt that the permeability of many plasma membranes, especially of irritable cells, is very sensitive to changes of electrical condition; thus the turgor motors of plants (Mimosa, Dionca) depend for their action upon variations of permeability, which are readily induced by the electric current. The passage of a current through a muscle or nerve, in such a way as to decrease locally the resting polarization of the cell-

× Cf. J. Loeb, Jour. Gen. Physiol., IV (1922), 463, and earlicr references there given.

${ }^{2}$ Girard, Jour. de Physiol. et de Path. gén., loc. cil. 
surface (i.e., cause depolarization), causes stimulation, which, as will be shown below, is attended with an increase of permeability. We must conclude that electrical factors are of great importance in determining the properties of living plasma membranes; variations in the external electrical conditions occasion corresponding variations in the membrane, and with these are connected various physiological effects. The polar disintegration shown by various cells through which currents are passed also indicates a direct action on the membrane; the character of this action is determined by the direction of the current. The law of polar stimulation is the expression of similar conditions in irritable tissues. ${ }^{x}$ The physiological effects of salts and electrolytes are undoubtedly to be referred largely to changes in electrical conditions, as shown by the numerous parallels between the action of salts in purely physical phenomena like electrical endosmose or change of colloidal aggregation, and their action on vital activities of various kinds.

I Cf. chapter on stimulation. 


\section{CHAPTER VIII}

\section{RELATION OF THE INORGANIC SALTS OF THE MEDIUM TO THE PHYSIOLOGICAL PROCESSIS IN PROTOPLASM}

The relation of the inorganic salts present in the external medium to the properties and activities of living cells has been the subject of much investigation since Ringer's time, and the present brief account will be confined to the more general and fundamental relations of this kind, especially those indicating that the chief basis of the physiological action of the salts of the medium is their action upon the protoplasmic surface layers. Since much of the pioneer work in this field is due to Overton, and since many of the results of his studies are applicable to living protoplasm in general. I shall first give a somewhat detailed summary of his earlier experiments on the action of salt solutions on the muscle and nerve of frogs. ${ }^{\mathrm{T}}$

Overton first examined carefully the well-known effect, reversible loss of irritability in isotonic solutions of indifferent non-electrolytes such as sugar, using small muscles; e.g., sartorius, cutaneus pectoris, and foot muscles. He found that all indifferent non-electrolytes, independently of their special composition (dextrose, sucrose, lactose, erythrite, mannite, alanin, taurin, and asparagin), produce this effect. The action is not poisonous, since the addition of a small proportion of an isotonic solution of sodium chloride to the non-electrolyte

${ }^{3}$ Overton, Arch. ges. Physiol., XCII (1902), 346; CV (1904), 170. 
solution prevents the loss of irritability; the inference is therefore justified that the effect depends upon a withdrawal of electrolytes, especially sodium chloride, from the tissue. That the electrolytes thus removed come almost entirely from the interstitial spaces of the tissue, and not from the interior of the cells, was later proved by Urano and Fahr. ${ }^{\mathrm{I}}$ The important conclusion follows that the presence of electrolytes (salts) in the external medium is essential to the normal irritability of the cell.

Overton discusses the question whether the removal of sodium salts acts by preventing the conduction of stimulation or by deranging the contractile mechanism of the cell. Biedermann had previously shown that after incorporation of sufficient water in hypotonic salt solution, a muscle may lose the power of contraction without losing that of conducting stimuli. ${ }^{2}$ By immersing a portion of a sartorius in isotonic sugar solution, Overton showed that stimuli are not transmitted through the salt-free muscle. It is known that a muscle deprived of irritability in sugar solution will shorten in solutions of chloroform or other cytolytic substances; presumably, therefore, the contractile mechanism is structurally intact, but fails to act in the absence of electrolytes because of the failure of conduction. Without the power of transmitting stimuli, the muscle is unable to contract as a whole.

The least concentration of $\mathrm{NaCl}$ required for the maintenance of irritability was determined by using mixtures of isotonic sugar solution (6 per cent) and

'Urano, Z. Biol., L (1907), 212; LI (1908), 483; Fahr, Z. Biol., LII, 72.

${ }^{2}$ Biedermann, Ber. Akad. Wiss. Wien, XCVII (I888), гог. 
$\mathrm{NaCl}$ solution (0.7 per cent). No significant decline in irritability was found until the salt-content fell below O.I 5 per cent. At O.I per cent irritability was distinctly less than normal, and, with further decrease in concentration, it declined rapidly, becoming zero at about 0.07 per cent. In mixtures containing less than 0.07 per cent $\mathrm{NaCl}$ irritability was lost as rapidly as in pure sugar solution. ${ }^{x}$ The time required for complete loss of irritability thus represents the time required for diffusion to reduce the $\mathrm{NaCl}$ of the intercellular spaces to this concentration.

Sugar-treated muscles rapidly regain irritability in solutions of all $\mathrm{Na}$ salts. The nature of the anion associated with the $\mathrm{Na}$ was found to be indifferent, provided it was not too toxic; with most salts the minimal concentration for the maintenance of irritability was about the same as with $\mathrm{NaCl}$. Apparently, therefore, it is the $\mathrm{Na}$ ion, and not the undissociated molecule or the anion, which is responsible for the maintenance of irritability. A definite function, that of preserving the normal irritability of muscle and nerve, is thus to be ascribed to the $\mathrm{Na}$ salts in blood plasma; their rôle is not merely osmotic, as formerly supposed. ${ }^{2}$ This relation of $\mathrm{Na}$ ions to irritability and contractility is a specific peculiarity of muscle cells; the conditions in other contractile forms of protoplasm are often widely different; thus the contraction of cilia, spermatozoa

${ }^{1}$ This effect apparently varies with oxygen tension. Pond has recently shown that in solutions saturated with oxygen the $\mathrm{NaCl}$ content may be reduced to less than .05 per cent without loss of irritability: Jour. Gen. Physiol., III (I92I), 807.

${ }^{2}$ Cf. also J. Loeb, "On the Production of Rhythmical Contractions in Muscles by Ions," Festschrift für Fick (1899), p. ror. 
and protozoan structures like the stalk of Vorticella is independent of $\mathrm{Na}$ ions in the medium; cilia, in fact, often exhibit prolonged and vigorous activity in media, like isotonic $\mathrm{KCl}$ or $\mathrm{MgCl}_{2}$ which rapidly and completely abolish irritability in muscle and nerve. ${ }^{\mathrm{T}}$

Since analysis shows little or no sodium in the interior of the muscle cells, the conclusion follows that the essential action of the $\mathrm{Na}$ ions is exerted upon the semi-permeable surface layer or plasma membrane and that penetration into the internal protoplasm is unnecessary. Overton, however, points out that it is not necessary to assume impermeability to these ions under all conditions; it is possible that under some conditions, e.g., stimulation, there may be an exchange between the $\mathrm{Na}$ ions in the medium and other cations (e.g., K) present in the muscle cell. This suggests that impermeability to $\mathrm{Na}$ ions is a characteristic of the muscle during the resting state only, a view implying that changes of permeability are an essential factor in stimulation. Similar views were expressed by Bernstein and Brünings at about the same time. ${ }^{2}$

Experiments on the substitution of other cations for $\mathrm{Na}$ showed that lithium salts were the only ones capable of maintaining irritability in the same manner as $\mathrm{Na}$ salts. Pure isotonic solutions of $\mathrm{LiCl}$ ( 0.435 per cent) are injurious to muscle, but a mixture of this solution with an equal volume of isotonic sugar solution has indifferent properties like those of an $\mathrm{NaCl}$ solution. The least concentration of $\mathrm{LiCl}$ required for maintaining

${ }^{x}$ The cilia of many marine animals, e.g., Arenicola and Mitylus, remain active for hours in pure solutions of $\mathrm{K}$ and $\mathrm{Mg}$ salts.

${ }^{2}$ Bernstein, Arch. ges. Physiol., XCII (1902), 521; Brünings, ibid., XCVIII (I903), 24I, and C, 367 . 
irritability is $c a 0.05$ per cent, a value comparable with that found for $\mathrm{NaCl}$. Lithium chloride, nitrate, sulphate, phosphate, and acetate all showed similar action. All salts of the other alkali cations $\left(\mathrm{NH}_{4}, \mathrm{~K}\right.$, $\mathrm{Rb}$, and $\mathrm{Cs}$ ), in concentrations equivalent to 0.07 per cent $\mathrm{NaCl}$, promptly destroyed irritability. $\mathrm{NaCl}$ solutions containing a little $\mathrm{K}$ and $\mathrm{Ca}$ (Ringer's solution) were more effective in reviving irritability than pure $\mathrm{NaCl}$ solutions; irritability, however, was not sustained by solutions containing $\mathrm{Ca}$ and $\mathrm{K}$ in the same proportions, but sugar in place of $\mathrm{NaCl}$. $\mathrm{Na}$ and $\mathrm{Ca}$ salts, especially when both are present in the solution, antagonize the toxic action of potassium salts; this is readily shown by using concentrations of $\mathrm{KCl}$, which, acting in pure solution, rapidly destroy irritability.

The addition of traces of $\mathrm{CaCl}_{2}$ and $\mathrm{SrCl}_{2}$ to isotonic sugar solutions was found to delay the loss of irritability, and a slight revival was observed when sugar-muscles were returned to weak solutions of $\mathrm{Ca}, \mathrm{Sr}, \mathrm{Ba}$, or $\mathrm{Mg}$ salts in isotonic sugar solution; but no restorative action was observed in pure isotonic solutions of these salts.

The results of experiments with nerve-trunks were on the whole similar to those with muscle, ${ }^{1}$ although on account of the structure of nerve a much longer immersion in sugar solution (in the cold) was necessary in order to abolish irritability completely. The minimal concentration of $\mathrm{Na}$ salts for preserving irritability proved to be about the same as in muscle; on nerve as well as on muscle $\mathrm{K}$ salts have a characteristic paralyzing action, which is also antagonized by $\mathrm{Ca}$ and $\mathrm{Sr}$ in the presence of $\mathrm{Na}$ salts.

I Overton, loc. cit. (1904). 
An important section of Overton's work has reference to the influence of salts on the transmission across the myoneural junctions and through reflex arcs. Locke ${ }^{x}$ (I894) had found that when the frog's sartorius, with nerve attached, was placed in a pure isotonic $\mathrm{NaCl}$ solution the tissue within fifteen to twenty minutes lost irritability through the nerve for single induction shocks, while it remained directly irritable for some hours; the addition of a little $\mathrm{CaCl}_{2}$ ( 0.02 per cent) to the solution restored indirect irritability in a few minutes; a second return to pure $\mathrm{NaCl}$ again abolished stimulation through the nerve, and the effect could be again reversed by $\mathrm{CaCl}_{2}$. Muscles perfused with salt solution show the same phenomenon; ${ }^{2}$ and other cases of innervation, such as inhibition of the heart through the vagus, are similarly dependent on the salts of the medium. ${ }^{3}$

Overton ${ }^{4}$ found that the addition of $\mathrm{KCl}$ to the $\mathrm{NaCl}$ solution greatly accelerated the junctional paralysis; salts of $\mathrm{Rb}$ and $\mathrm{Cs}$ acted similarly, also salts of $\mathrm{NH}_{3}$ and organic ammonium compounds, which have long been known to exhibit this curare-like action. When the concentration of $\mathrm{NaCl}$ was 0.7 per cent, the addition of .05 per cent $\mathrm{KCl}$ was found to shorten the period of irritability through the nerve to an eighth or a tenth of its duration in the pure solution; with a lower concentration of $\mathrm{NaCl}$ less $\mathrm{KCl}$ was required. This blocking effect of potassium is antagonized by calcium; there is,

${ }^{x}$ Locke, Zentralbl.f. Physiol., VIII (I894), 166.

${ }^{2}$ Cushing, American Journal of Physiology, VI (IgOI), 77.

${ }^{3}$ Cf. Höber, op. cit., p. 539; Howell, American Journal of Physiology, XV (1906), 280; Howell and Duke, ibid., XXXV (1907), I31.

4 Loc. cit. 
in fact, enough $\mathrm{K}$ in blood plasma to destroy all myoneural transmission, were it not for the $\mathrm{Ca}$ also present. Calcium is the only metallic ion in plasma (other than $\mathrm{Na}$ ) required for myoneural transmission; in mixtures of $\mathrm{NaCl}$ and $\mathrm{CaCl}_{2}$ indirect irritability is preserved almost as long as in serum. Sr also antagonizes this action of $\mathrm{K}$, but $\mathrm{Ba}$ and $\mathrm{Mg}$ do not. A similar blocking of myoneural transmission is caused by $\mathrm{BaCl}_{2}$; this action is also antagonized by $\mathrm{Ca}$. Overton found that in a mixed solution of sodium and calcium chlorides of the same concentration as in serum ( 0.7 per cent $\mathrm{NaCl}$ plus 0.02 to $0.03 \mathrm{CaCl}_{2}$ ) the addition of 0.05 to 0.06 per cent $\mathrm{KCl}$ completely paralyzed the nerve-endings without affecting the direct irritability of the muscle; in order to abolish the latter O.I5 per cent $\mathrm{KCl}$ was required; when more $\mathrm{CaCl}_{2}$ was added to the solution more $\mathrm{KCl}$ was required to prevent transmission. In the absence of $\mathrm{K}$ a mere trace of $\mathrm{Ca}$ is all that is necessary; in a $\mathrm{K}$-free $\mathrm{NaCl}$ solution one part of $\mathrm{CaCl}_{2}$ in 20,000 maintained indirect irritability for twenty-four hours and one part in 50,000 for twelve hours; even one part in 100,000 had a perceptible effect.

Although the nerve-trunk retains its power of conduction for many hours in pure solutions of $\mathrm{Na}$ and $\mathrm{Li}$ salts, transmission through reflex arcs is quickly prevented by lack of $\mathrm{Ca}^{\mathrm{I}}$ This was shown both in perfusion experiments with intact frogs and in experiments in which the isolated spinal cord with nerves and muscles attached was immersed in the solution. In such a preparation kept at a low temperature in well-oxygenated

'Overton, Verhandl. Ges. deutscher Naturf. u. Ärze, LAXY' (1)0,3), Theil II, 2te Hälfte, p. $4 \mathrm{I} 6$. 
Ringer's solution reflex activity may continue for days. If the cord and attached sciatic nerves are immersed in isotonic sugar solution, reflexes completely disappear within twenty-four to thirty-six hours; on returning the cord to Ringer's solution they return in a few hours. In perfused frogs, replacement of the blood by sugar solution rapidly causes disappearance of reflexes and later of direct muscular irritability: perfusion with pure $\mathrm{NaCl}$ solution then restores direct muscular irritability, but not indirect or reflex, while Ringer's solution restores all three. The quantity of $\mathrm{Ca}$ necessary for restoring reflexes (as well as indirect irritability) is very slight.

In the observations above most of the fundamental phenomena of salt action are illustrated-the necessity of salts for cellular activity, the toxic action of pure solutions, ion-antagonisms, and especially the remarkable rôle of calcium in maintaining the normal structural and functional relationships between cells. With regard to this latter phenomenon, Overton cites Herbst's observations on the action of calcium in promoting the coherence of blastomeres, ${ }^{\mathrm{I}}$ and also calls attention to the presence of this element in the middle lamella of plant tissues, where, according to Mangin, it is present as a compound, Ca-pectate (or pectinate), which is necessary for intercellular coherence. When this compound is removed (by weak acid) or when it is substituted by the $\mathrm{Na}$ salt (e.g., in pure $\mathrm{NaCl}$ solution) the cells fall apart. ${ }^{2}$ Apparently some Ca compound is necessary for the

'Herbst, Arch. Entwicklungsmech., IX (I900), 424.

${ }^{2}$ Compare the recent observations of Hansteen on plant tissues; again pure $\mathrm{NaCl}$ solutions cause a disintegration due to loss of intercellular coherence: Jahrb. wiss. Botanik, XLVII (I9Iо), 289; LIII (I9r4), 536. 
intimate union between the nerve end-plate and muscle cell, or for the normal properties of the synaptic junctions. Overton suggests that when the tissue is transferred to pure $\mathrm{NaCl}$ solution, the $\mathrm{Ca}$ in this compound is replaced by $\mathrm{Na}$, producing a compound of greater water-absorbing properties, which swells and interrupts the union. Such an effect is reversed by a return to Ca-containing solutions.

Some of the more general inferences to be drawn from these and recent experiments of a similar kind are as follows: Since irritability disappears in isotonic sugar solutions, which maintain the osmotic balance without apparently changing the crystalloid content of the cell. it seems clear that the action of the salts of the external medium must be superficial; i.e., is a surface-action exerted upon the plasma membrane. This action is probably of a twofold nature: first, the normal composition and physical properties of the colloidal materials composing the surface-film are preserved only when a certain combination of ions is present in the medium; and, second, the normal state of electrical polarization of the membrane is also dependent on the presence of salts in the medium as well as in the cell-interior; this effect is important because the electrical polarization of the membrane is a factor determining its permeability. These two effects, however, cannot be regarded as independent, since changes in the physical state of the membrane must alter its permeability and (in so doing) its electrical polarization; and, conversely, changing the electrical polarization influences the permeability.

The essential conclusion, however, is that salts may exert physiological action without penetrating into the 
interior of the cell, solely by altering the state of the plasma membrane, and there is much additional evidence that this is the case. The analytical results of Urano and $\mathrm{Fahr}^{\mathrm{I}}$ show that in normal vertebrate muscle $\mathrm{Na}$ is almost entirely absent from the cell interior, although, as just shown, it is essential to the maintenance of irritability. Urano kept frogs' sartorii for several hours in isotonic sugar solution, and found that the muscle gave off to the solution relatively much more $\mathrm{Na}$ than $\mathrm{K}$. Muscles thoroughly extracted in sugar solution were found to contain only 2 per cent or less of the normal total Nacontent, but nearly all of the $\mathrm{K}$ and phosphate. The conclusion seems certain that the $\mathrm{Na}$ is contained almost entirely in the intercellular spaces of the tissue, and the $\mathrm{K}$ and phosphate in the cells. Muscles kept for six hours in sugar solution lose very little ash that may not be accounted for by the salts present in the interstitial lymph. Fahr, in an ash-analysis of the extract of fresh uninjured muscles in isotonic sugar solution, found that only 6 per cent of the original $\mathrm{K}$ of the tissue, but 90 per cent of the original $\mathrm{Na}$, was thus recoverable. If four-fifths of the total volume of the muscle be regarded as consisting of muscle cells, and one-fifth of interstitial tissue and lymph spaces, the Na-content of the tissue is completely accounted for by the salts present in the lymph. The latter must therefore exert their physiological influence through their action upon the external protoplasmic layer or plasma membrane.

Höber's experiments on the influence of isotonic solutions of neutral salts on the demarcation-current potential of muscle ${ }^{2}$ also support Overton's view that

${ }^{x}$ Loc. cit. $\quad{ }^{2}$ Höber, Arch. ges. Physiol., CVI (1905), 599. 
the normal uninjured resting muscle is impermeable to sodium salts. Solutions of sodium and lithium salts leave the normal electrical potential of the external muscle-surface unchanged; while salts like those of $K$, $\mathrm{Rb}$ and $\mathrm{NH}_{4}$ (which give other evidence of penetrating the muscle) produce an injury-current or local negativity. Absence of penetration is indicated by failure to change the external potential of the muscle; the alkali earth salts and in part those of cæsium are thus indifferent. Nevertheless, they alter the properties of the tissue; thus $\mathrm{Mg}$ salts have a strongly anti-stimulating or narcotic action, and $\mathrm{Ca}$ and $\mathrm{Sr}$ salts in pure isotonic solution similarly render muscle resistant to stimulation; all of these effects are reversible if the exposures are not too prolonged.

Loss of contractility in non-electrolyte solutions and its prompt return in solutions of sodium salts-especially if some $\mathrm{Ca}$ is also present-are characteristic of many varieties of muscle. Among invertebrates the larvæ of Arenicola show this phenomenon in a striking manner; here also, as with vertebrate muscle, $\mathrm{Li}$ and $\mathrm{Na}$ salts are alone capable of preserving contractility for prolonged periods, and $\mathrm{K}$ salts have a paralyzing action. Mg salts repress contractility very promptly and completely. and the action is readily reversed, especially by solutions of $\mathrm{Na}$ salts containing a little $\mathrm{Ca}^{2}{ }^{2}$ The resemblance of $\mathrm{Li}$ to $\mathrm{Na}$ in its power of maintaining the normal properties of muscle (though less perfectly than $\mathrm{Na}$ ) seems to be general for both vertebrate and invertebrate muscle; for example, Mines describes this phenomenon in the

${ }^{2}$ R. S. Lillie, American Journal of Physiology, XXIV (rgog), 459.

${ }^{2}$ Op. cit., p. 485. 
molluscan heart $(\text { Pecten })^{\mathrm{x}}$ and in the heart of elasmobranch fishes.

Much further evidence could be cited indicating that changes in the plasma membranes of the living cells, produced by salts or other compounds which show no evidence of penetrating into the cell interior, may profoundly affect the properties of irritable tissues or organisms. An interesting example is seen in the changes produced by isotonic salt solutions in the sensitivity of frog's muscle to various forms of chemical stimuli and to physical agents like heat or contact. A curarized frog's gastrocnemius, placed for a few minutes in a pure isotonic solution of a sodium salt $\left(\mathrm{NaCl}, \mathrm{NaBr}, \mathrm{NaNO}_{3}, \mathrm{NaI}, \mathrm{NaClO}_{3}\right.$, etc.) and then dipped into a solution containing a stimulating compound ( $\mathrm{K}$ salt) or a cytolytic agent (chloroform in saturated solution), contracts much more vigorously than a normal muscle which is dipped into the same solution directly from Ringer's solution. The pure $\mathrm{Na}$ salt solution increases the responsiveness to the stimulating agent; i.e., induces a sensitization; this effect is readily antagonized by $\mathrm{CaCl}_{2}$. The reverse effect (desensitization) is produced by exposure to isotonic $\mathrm{CaCl}_{2}, \mathrm{SrCl}_{2}$, or $\mathrm{MgCl}_{2}$; after a brief stay in these solutions the muscle fails to respond to the stimulating solution or responds subnormally. In all such cases normal irritability returns in Ringer's solution. ${ }^{2}$ Various facts indicate that $\mathrm{Ca}$ compounds in the surface layer of the cell are

${ }^{I}$ G. R. Mines, Journal of Physiology, XLIII (I9I2), 467.

${ }^{2}$ R. S. Lillie, Proceedings of the Society of Experimental Biology and Medicine, VI (I910), I70; American Journal of Physiology, XXVIII (I9II), 197; cf. p. 215. 
concerned in normal irritability; a striking example is the marked hypersensitivity induced in frog's muscle and nerve by $\mathrm{Na}$ salts whose anions precipitate calcium or form Ca salts of limited solubility. Muscles treated for a few minutes with isotonic solutions of these salts (sulphate, phosphate, tartrate, citrate, oxalate, etc.) become highly sensitive to the contact of foreign substances or media, and contract vigorously when exposed to air. ${ }^{2}$ These phenomena of sensitization are produced so rapidly as to leave little doubt that they depend on alterations in the surface-films of the cells. ${ }^{3}$

Direct evidence that changes in the permeability of the plasma membranes play an essential part in processes of stimulation will be cited later. Apparently all substances that alter the physical or chemical state of the plasma membrane (salts, acids, alkalis, narcotic agents) modify the stimulation-process; this influence may be in the direction either of facilitation or of repression, according to conditions. ${ }^{4}$

The precise means by which salts produce these effects has been much debated and is still imperfectly

${ }^{2} \mathrm{Cf}$. the experiments of $\mathrm{A}$. J. Clark, cited on page I 85 .

${ }^{2} \mathrm{~J}$. Loeb, American Journal of Physiology, V (I

3 Other effects dependent on surface changes in the cells are related to those just described; e.g., changes in the surface-films of blood corpuscles and bacteria, affecting the critical concentrations of hiemolysis or agglutination by $\mathrm{H}$ ions, are produced by the addition of proteins; cf. the recent papers of Coulter, Jour. Gen. Physiol., III (1921), 309, and IV (1922), 403; Northrop and De Kruif, ibid., IV (1922), 655; also Eggerth and Bellows, ibid., p. 669.

${ }^{4}$ Such effects evidently imply an influence on cell metabolism. Warburg's observations on sea-urchin eggs show that the oxygen consumption may be increased several times by the addition of alkali which shows no evidence of penetrating the cell: $Z$. physiol. Chem., LAVI (1910), 305 . 
understood. Perhaps the clearest light on the problem has been afforded by the phenomena of salt-antagonism. These have shown definitely, at least in certain cases, that structural changes in those parts of the cell which are most directly exposed to the action of the solution (plasma membranes and other surface-structures like cilia) are the primary condition of the effects produced. The toxic and other effects are secondary consequences of these structural changes. The physical condition of the structural colloids-state of subdivision, of hydration, of electrical polarization, etc.-is changed by the action of the salt, with corresponding changes in the properties of the cell-structure itself and of the chemical and other activities controlled by it. In many cases the injurious action of the pure salt-solution $(\mathrm{NaCl})$ is referable to a destruction of the normal semi-permeability of the plasma membrane; this effect, if not soon reversed, involves chemical and other disorganization followed by death. Any condition preventing or retarding the alterative action on the membrane, such as the presence of an antagonistic salt or non-electrolyte (narcotic), has accordingly a protective or "anti-toxic" action. ' These general conditions are well illustrated in a simple marine organism much used in experimental work at Woods Hole, the larva of the annelid Arenicola cristata. This is a segmented trochophore larva about one-third of a millimeter in length, having pigmented body cells and swimming by a combination of muscular and ciliary movements. When placed in pure isotonic

${ }^{1}$ Cf. the discussion in my paper on antagonism between salts and anæsthetics, American Journal of Physiology, XXXI (19r3), 255; cf. pp. $275 \mathrm{ff}$. 
$\mathrm{NaCl}$ (or similar unbalanced solution of alkali salt) the larva contract strongly and the yellow pigment begins to diffuse from the cells; by degrees the cilia cease movement and undergo a visible breakdown or disintegration (suggesting liquefaction or absorption of water). In a solution of $\mathrm{NaCl}$ containing a little $\mathrm{CaCl}_{2}$ (95 volume $\mathrm{m} / 2 \mathrm{NaCl}$ plus 5 vols. $\mathrm{m} / 2 \mathrm{CaCl}_{2}$ ) all of these immediate effects are prevented, and the larvic retain their normal appearance and behavior for some time and die much more gradually. ${ }^{x}$

In this case it is clear that the immediate action of the pure salt-solution is upon the surface structurescilia and plasma membranes - which quickly lose their normal structural coherence and continuity; the result of this change in the cilia is physical breakdown, and in the plasma membrane a marked increase of permeability, hence the diffusion of soluble cell-constituents to the exterior and the progressive disorganization. In many other organisms and cells it can also be shown that an abnormal increase of permeability is produced by pure solutions of $\mathrm{Na}$ salts and prevented by the addition of $\mathrm{CaCl}_{2}$ or other antagonistic salt. ${ }^{2}$ The experiments of Osterhout on the electrical conductivity of Laminaria and other plant tissues afford perhaps the clearest evidence that the toxic action of pure $\mathrm{NaCl}$ solutions is the result of a destruction of semi-permeability and that antagonistic salts $\left(\mathrm{CaCl}_{2}\right.$ and others) produce their anti-

I American Journal of Physiology, XXIV (Igog), I.t; cf. pages 22 fi.

${ }^{2}$ In an earlier paper (Biological Bulletin, XVII [1909], ISS) I have given a summary of observations showing the correlation between permeability-increasing action and toxicity for compounds other than salts. 
toxic effects by counteracting the structural change in the membrane. ${ }^{\mathrm{I}}$

The question of just how, in the physico-chemical sense this result is accomplished is a fundamental one; salt antagonism is shown by all groups of organisms from bacteria $^{2}$ to vertebrata, and is apparently a universal phenomenon in living protoplasm. It should be noted that other effects produced by the pure $\mathrm{Na}$ salt solution are also antagonized by $\mathrm{Ca}$ and other salts; e.g., its stimulating action, shown in the production of twitches in vertebrate and other muscle, ${ }^{3}$ the sensitizing action on muscle just described, and the activation of unfertilized eggs. ${ }^{4}$ The fact that prevention (by $\mathrm{Ca}$ and narcotics) of increase of permeability in various irritable tissues and organisms is associated with prevention of stimulation has a general interest as evidence of the essential part played by membranes in stimulation, and will be considered more fully later.

The problem of the physico-chemical basis of salt antagonism has been approached in various ways and

I Osterhout, loc. cit. Related observations are those of Hamburger on the action of calcium in preventing the increase in permeability produced in frog's kidneys perfused by $\mathrm{NaCl}$ solutions containing dextrose (cf. Hamburger, Biochem. Zeitschrift, LXXXVIII [19I8], 97). In I904 J. B. MacCallum had observed the antidiuretic action of calcium salts and had related it to the action in decreasing permeability (Journal of Experimental Zoölogy, I [1904], I79; University of California Publications, Physiol., II [I905], 93). It is well known that calcium antagonizes cytolysis by saponin and other permeability-increasing compounds. Chiari's observations on the formation of exudates and many other observations are related; cf. Höber, op. cit., pp. $544 \mathrm{ff}$.

${ }^{2}$ For the case of bacteria cf. Shearer, Journal of Hygiene, XVIII (1919), 339.

${ }^{3}$ Cf. Loeb's article in Festschrift für Fick, loc. cit.

4 R. S. Lillie, American Journal of Physiology, XXVII (IgI I), 289; Journal of Morphology, XXII (I9II), 695. 
on the whole most effectively by comparing the action of salts on colloidal solutions and emulsions with their action on living cells. ${ }^{\mathrm{I}}$ The physiological effects produced by salts and salt combinations vary with the nature of the ions in a manner which in many cases shows a close parallelism with the physical effects produced by the same salts in simple colloidal systems. For example, in the counteraction of the toxic effects of pure $\mathrm{Na}$ salt solutions the results all indicate that the cation of the antagonistic salt is the effective agent; and in this case certain relations highly characteristic of the action of ions on colloidal systems are shown clearly; thus with the cilia of Arenicola all salts of bivalent heary metals ( $\mathrm{Co}, \mathrm{Ni}, \mathrm{Cd}, \mathrm{Zn}, \mathrm{Mn}, \mathrm{Pb}, \mathrm{Fe}^{++}, \mathrm{Cu}$ ) were found to produce their maximal antitoxic effects in concentrations of the order $\mathrm{m} / 400$ to $\mathrm{m} / \mathrm{I} 600$; while with trivalent metals $\left(\mathrm{Al}, \mathrm{Cr}, \mathrm{Fe}^{\prime \prime \prime}\right)$ the physiologically corresponding concentrations were from 50 to roo times less. ${ }^{2}$ This increase in effectiveness with increase in valence is characteristic of the action of salts on negatively charged suspensoid systems (rule of Schulze and Hardy); and apparently the observations above indicate that the cations produce their effects in the living system by influencing the state of subdivision of the suspensoid colloids forming part of the protoplasmic structure. Other cases of rapid increase in physiological effectiveness with increase of valence are well known.

Such cases of antagonism are consistent with the hypothesis that the normal state of the living protoplasmic

${ }^{x}$ Cf. Höber's review in his textbook, chap. x, p. 47 I.

${ }^{2}$ American Journal of Physiology, VII (1904), $4 \mathrm{rg}$; similar results were obtained with the gill cilia of MFitylus: ibid., XIII (190(i), Sy. 
structure (e.g., a cilium or plasma membrane) requires a certain state of subdivision of the chief structural colloids. Apparently in the normal medium, e.g., sea water, the influence of the various ions is so balanced as to preserve this state; the collective effect of the negative charges carried by the anions $\left(\mathrm{Cl}, \mathrm{SO}_{4}, \mathrm{HCO}_{3}\right.$, etc. $)$ is just balanced by the collective effect of the positive charges carried by the cations ( $\mathrm{Na}, \mathrm{K}, \mathrm{Ca}, \mathrm{Mg}$ ). In the pure solution, however, of $\mathrm{NaCl}$, in which no bivalent cations are present, the influence of the anions is insufficiently compensated, and the colloids are altered in a manner injurious to cell structure: i.e., a preponderant influence of anions is the essential toxic factor in such cases. The addition of bivalent or trivalent cations of any kind has under these conditions a compensating and hence antagonistic or antitoxic influence; trivalent ions exercise this effect in much lower dilution than bivalent ions, and bivalent than monovalent ions. At an appropriate concentration, the balance is restored, protoplasmic structure remains normal, and life continues. The reverse condition, in which the toxic action of the pure solution results from a prepotency of cation action, and in which accordingly the addition of small quantities of salts with powerfully acting anions has an antitoxic effect, is apparently also realized in some cases; e.g., the ciliated epithelium of the molluscan gill (Mitylus) in solutions of $\mathrm{SrCl}_{2}$; here various $\mathrm{Na}$ salts $(\mathrm{NaOH}, \mathrm{NaBr}, \mathrm{NaI}$, $\mathrm{NaCNS}, \mathrm{Na}_{2} \mathrm{SO}_{4}$ ) show well-marked antagonistic action. ${ }^{\mathrm{x}}$ Such purely physical explanations, while apparently partly applicable in some cases, prove inadequate in

${ }^{x}$ Cf. American Journal of Physiology, XVII (1906), 89; cf. p. I29; cf. Raber, Jour. Gen. Physiol., II (I920), 541, for analogous observations on Laminaria. 
many others, where the foregoing relation of antagonism to valence does not appear to hold, and especially in those numerous cases where different cations similar in valence have widely different antagonistic or other physiological actions. ${ }^{\mathrm{I}} \quad$ In such cases the special chemical properties of the compounds formed between the ions and the structural colloids of the cell must apparently be taken into consideration. Thus various facts indicate that calcium proteinates or calcium soaps (or both) are of special importance as constituents of cell structures; and these compounds cannot be replaced satisfactorily by the corresponding compounds of other bivalent elements. ${ }^{2}$ Such a conception explains why strontium, the metal most closely resembling calcium in general chemical properties, usually comes nearest to calcium in antagonistic effectiveness; the other alkali earth cations come next; while the bivalent heavy metals are effective in only a few special cases $\left(\mathrm{Mn} . \mathrm{Co}, \mathrm{Fe}^{++}\right)$, the great majority of these metals being too strongly toxic to act as efficient antagonists. ${ }^{3}$

${ }^{x}$ Cf. Höber's discussion in his recent papers on the physiological action of calcium; Arch. ges. Physiol., CLXVI (1917), 531, and CLXXXII (1920), 104 .

${ }^{2}$ The cases are numerous where calcium has a specific action which cannot be replaced by that of other cations, even $\mathrm{Sr}$; instances are the specificity of calcium in phagocytosis (Hamburger), in the inhibition of swimming plates, and in the preservation of contractility in heartstrips. On the other hand, in its simple stabilizing or protective action, (e.g., in cytolysis, etc.) Ca can often be replaced by other cations (cf. Höber's papers, loc. cit.; cf. also Wiechmann, Arch. ges. Physiol., CXCV [1922], 588).

${ }_{3}$ Nevertheless even the most toxic cations, like $\mathrm{Ag}$ and $\mathrm{Hg}$, may show definite antitoxic action in certain cases; e.g., the beat of the ctenophore swimming plate in pure isotonic $\mathrm{NaCl}$ solution is prolonged several times by $\mathrm{AgNO}_{3}$ and $\mathrm{HgCl}_{2}$ in concentrations of $\mathrm{m} / \mathrm{100,000}$ to $\mathrm{m} / 200,000$ (cf. American Journal of Physiology, XVI [1906], 117). 
The evidence, taken as a whole, indicates that the salts act chiefly by altering the physical state of the structural colloids of the cell; and it is to be presumed that general physical factors (of the kind regarded as acting in all colloidal phenomena) and special chemical factors specific for each form of protoplasm are both concerned in producing the total effect. In the directly toxic or injurious action of salt solutions, a frequent, if not invariable, factor is a destruction of the semipermeable properties of the protoplasmic partitions, primarily of the plasma membranes. In physiological salt-actions of other kinds, e.g., stimulation, sensitization, inhibition, it is to be presumed that the physical properties of the plasma membranes undergo special modifications of a corresponding kind, but that the effects do not exceed a certain range and are therefore reversible. The general fact that a certain combination of salts, usually of $\mathrm{Na}, \mathrm{Ca}$, and $\mathrm{K}$, is required in the external medium for most forms of normal protoplasmic action indicates the fundamental importance of the influence of salts on the structural colloids of protoplasm. Apparently the structural conditions required for the continuity and closeness of texture necessary in semi-permeable membranes depend on the maintenance of a definite equilibrium between the ions in the medium and those associated (chemically or otherwise) with the structural colloids. Slight changes in the salt-content imply corresponding structural changes, with dependent physiological effects.

It should be added that other general physical conditions, also dependent on the presence of salts, are of importance in the normal activity of protoplasm, 
such as the electrical conductivity of protoplasm and medium and the electrical polarization of the plasma membranes. These conditions will be considered later under the subject of stimulation.

The special relations of the three chief cations of the protoplasmic media, $\mathrm{Na}, \mathrm{K}$, and $\mathrm{Ca}$, to protoplasmic activity appear to depend on chemical conditions of a kind still imperfectly understood. The differences between the physiological actions of $\mathrm{Na}$ (and $\mathrm{Li}$ ) and of $\mathrm{K}$ (and $\mathrm{Rb}$ and $\mathrm{Cs}$ ) in their relation to vertebrate muscle and nerve cannot be satisfactorily explained on the basis of the physico-chemical constants of these ions. $\mathrm{Na}$ and $\mathrm{K}$, though chemically closely similar, appear in many forms of protoplasm, e.g., vertebrate muscle, to act as physiological antagonists; in others their physiological differences are relatively slight; e.g., in the case of fish eggs (Fundulus) and sea-urchin eggs (Arbacia) isotonic $\mathrm{KCl}$ solution is even less toxic than $\mathrm{NaCl}$ solution; both solutions are antagonized by $\mathrm{Ca}$.

It is remarkable that the striated muscle cells of vertebrata appear to be readily permeable to some $\mathrm{K}$ salts $\left(\mathrm{KCl}, \mathrm{KBr}, \mathrm{KI}, \mathrm{KNO}_{3}\right)$ but not to others $\left(\mathrm{K}_{2} \mathrm{SO}_{4}\right.$. $\mathrm{K}$-tartrate, $\mathrm{K}$-phosphate); ${ }^{\mathrm{I}}$ in this respect $\mathrm{K}$ salts exhibit a striking contrast to $\mathrm{Na}$ salts. The special permeability to $\mathrm{KCl}$ is shown in frog's muscle by rapid increase in the weight of the tissue when it is immersed in isotonic solutions of this salt; similar conditions have been found by Seebeck ${ }^{2}$ in the frog's kidney, which, like muscle, shows normal semi-permeability toward $\mathrm{Na}$ and $\mathrm{Li}$ salts. The special physiological action of $\mathrm{K}$ is prob-

I Overton, Arch. ges. Physiol., CV (1904), I 76.

'Seebeck, ibid., CXLVIII (1912), 443. 
ably related to this peculiarity, but the nature of the relationship is unknown. Recently Zwaardemaker has attributed importance to the slight radioactivity of $\mathrm{K}$, on the ground of certain striking parallels between the action of $\mathrm{K}$ salts and uranium salts on the frog's heart; ${ }^{\mathrm{I}}$ but other evidence from experiments with marine organisms fails to support this view. ${ }^{2}$

Recently Meigs ${ }^{3}$ has investigated the osmotic behavior of smooth muscle in solutions of salts and non-electrolytes, and finds a number of remarkable differences between this tissue and striated muscle. For example, the stomach muscle of the frog gains weight when immersed in isotonic solutions of sugar, alanin, or $\mathrm{NaCl}$, and even in Ringer's solution. He concludes that smooth muscle cells differ fundamentally in their structure and mode of action from striated muscle cells, and that they are not surrounded by semi-permeable membranes. In the adductor muscle of the marine clam (Venus mercenaria), a smooth muscle which reacts slowly and maintains its state of tension for a long time, closely similar conditions were found. Pieces of muscle immersed in sea water take up chloride from the latter and increase in weight; they even fail to lose weight in Io per cent $\mathrm{NaCl}$ or in sea water of twice the normal concentration. ${ }^{4}$

× Zwaardemaker, Journal of Physiology, LIII (1920), 273, and LV (192I), 33 .

${ }^{2}$ R. F. Loeb, Journal of General Physiology, III (1920), 229. A. J. Clark fails to confirm Zwaardemaker's claim that uranium can act as a substitute for $\mathrm{K}$ in restoring the heartbeat (Journal of Pharmacology and Experimental Therapeutics, XVIII [1922], 423).

3 E. B. Meigs, Journal of Experimental Zoölogy, XIII (IgI 2), 497.

${ }_{4}^{4}$ Meigs, Journal of Biological Chemistry, XVII (I9I4), 8I. 
In explanation of these apparent discrepancies it has been suggested that smooth muscle cells are easily injured and that the normal semi-permeability had disappeared at the time when the tissue was examined, but this possibility is rejected by Meigs. It is difficult to escape the conclusion that a continuous semi-permeable membrane does not invest the whole cellular element in this tissue. Possibly the contractile part of the entire structure has a relation to the protoplasmic part similar to that which the fibers in connective tissue have to the cells by which they are formed. The sluggishness of the movement and its slow reversibility suggest a fundamentally different type of organization from that of striated muscle cells. Semi-permeability appears to be universal in indifferentiated cellular elements and in the majority of specialized cells; but a differentiation in which part of the total structure acquires non-cellular properties is not infrequent in organisms, as illustrated in connective tissues and skeletal structures; and the above-cited types of contractile tissue may exemplify this general condition.

The penetration of salts through the egg-membrane of the sea-minnow, Fundulus, as shown in various toxic effects and antagonisms, illustrates many conditions of great interest, and especially indicates the importance of the purely chemical factors in permeability. This membrane is a dead structure, or chorion, external to the living protoplasm of the egg, and apparently consisting chiefly of a keratin-like protein. In its normal state, it is almost completely impermeable to water or the salts of the medium; hence the eggs will develop either in distilled water or in concentrated sea water. 
Pure salt solutions $(\mathrm{NaCl})$ or solutions of acid or alkali alter the membrane and allow penetration. Characteristic antagonisms are shown in these effects; ${ }^{I}$ e.g., the penetration of heavy metal salts (cobalt and nickel) is retarded by $\mathrm{CaCl}_{2}$ and their toxic action is thus prevented. $^{2}$ Eggs placed in pure strongly hypertonic $\mathrm{NaCl}$ solutions float at first, but soon sink and die, indicating the penetration of salt and water as the membrane is altered; but in the same solution to which $\mathrm{CaCl}_{2}$ has been added, they may float and remain living for several days. ${ }^{3}$ Recently Loeb and Cattell have studied the penetration of $\mathrm{K}$ salts and acids into the Fundulus egg in the presence of other salts (alkali and alkali earth) in varying concentrations. ${ }^{4}$ As index of the penetration of $\mathrm{KCl}$ the paralyzing action of the salt on the heart was used; this action is reversible, so that if eggs containing embryos whose hearts have previously been arrested in $\mathrm{KCl}$ solution are placed in sea water, the heartbeat after a time revives, indicating outward diffusion of $\mathrm{KCl}$ through the membrane. The remarkable fact is that this recovery does not occur in distilled water or in solutions of non-electrolytes; in order that the potassium shall penetrate the membrane, a treatment of the latter with salt solutions is necessary. Thus (typically) all of the poisoned hearts resume beating within a day when the eggs are placed in sea water or

${ }^{x}$ Cf. the article by J. Loeb in Oppenheimer's Handbuch der Biochemie, II, I04, for a general account of antagonisms in Fundulus eggs.

${ }^{2}$ A. P. Mathews, American Journal of Physiology, XII (1905), 4I9.

${ }^{3}$ Loeb, Science, XXXVI (19I 2), 637; Biochem. Zeitschrift, XLVII (I912), I27.

${ }^{4}$ Loeb and Cattell, Journal of Biological Chemistry, XXIII (1915), 4I; Loeb, ibid., XXVII (1916), 339, 352, 363; XXVIII, I75. 
isotonic $\mathrm{NaCl}$ solutions, while none revive in sugar solution. A "salt action" is necessary to the penetration. of $\mathrm{KCl}$ in either direction through the membranc; all salts, however, are not equally effective; in the case of $\mathrm{Na}$ salts the effect shows an increase with increase in the valence of the anion. These effects apparently. indicate that the penetration of ions through a membrane consisting of colloidal material with which the ions form compounds (proteinates) requires the presence of other free ions with which the penetrating ion can form alternative combinations. Otherwise it is held in position by chemical forces and unable to move freely. Similar conditions have been observed in the diffusion of dyes (neutral red) from stained eggs; this diffusion is also facilitated by the presence of salts in the outer medium. In order to obtain the maximum diffusionfacilitating salt-effect a certain medium concentration of the effective salt is required; higher concentrations and lower concentrations have a retarding influence on diffusion; this latter effect appears to form the basis of the usual salt-antagonisms or protective effects shown by calcium and other bivalent salts. ${ }^{\mathrm{I}}$

It is evident that changes in the physical properties of membranes must alter the readiness with which diffusing materials penetrate, and that such changes may result from changes in the chemical composition of the membrane-forming compounds as well as from changes in their distribution or state of subdivision. In a membrane consisting of protein, the formation of proteinates with varying physical properties will change the permeability and the other properties of the membrane. Similarly:

${ }^{x}$ Loeb, Journal of Biological Chemislry (19I6), loc. cit. 
if lipoids or other ester-like compounds are membranecomponents, the formation of soaps becomes a possibility; these vary in their solubilities and watercombining properties; and variations in their proportions, e.g., the substitution of $\mathrm{Ca}$ soaps for $\mathrm{Na}$ or $\mathrm{K}$ soaps, will entail corresponding changes of permeability.

The question of whether the protein or the lipoid components of the living plasma membranes are chiefly concerned in the variations of permeability underlying toxic effects or normal physiological processes is an open one; but in all probability both compounds play a part, although the present evidence seems to favor the view that the proteins are physically the more stable of the two and form the permanent structural substratum, while variations of permeability depend chiefly on changes in the lipoid components, especially the soaps. Parallels to the physiological salt antagonisms are well known in non-living systems containing both classes of compounds. Of chief biological interest is the antagonism between $\mathrm{Na}$ salts and $\mathrm{Ca}$ salts, which is apparently universal in living organisms. Protein systems containing salts of these two metals show variations in properties when the proportions of the salts are varied; such properties as water-combining power (swelling or hydration), viscosity, osmotic pressure, susceptibility to alteration by organic compounds (precipitation by alcohol), and electrical polarization of particles are affected; Loeb's recent investigations afford instances of salt-antagonisms affecting all of these properties. ${ }^{x}$ The inference from such facts would be that $\mathrm{Ca}$ and $\mathrm{Na}$

${ }^{x}$ Loeb, Journal of Biological Chemisiry, XXXI (I9I7), 3; XXXIV (IgI8), 395, 489; XXXV (I9I8), 497. 
proteinates must co-exist in certain definite proportions in the cell-structures, including the membranes, if certain biologically necessary physical properties are to be preserved. Substitution of $\mathrm{Na}$ for $\mathrm{Ca}$ compounds would occur in pure $\mathrm{Na}$ salt solutions, with resulting structural changes which might well be injurious or fatal.

Clowes $^{\mathrm{I}}$ has recently shown that salt-antagonisms having an even closer resemblance to biological salt antagonisms may be demonstrated in oil-water emulsion systems by varying the proportions of $\mathrm{Na}$ and $\mathrm{Ca}$ soaps in the interfacial films. The number of separate drops formed when a slightly alkaline salt solution is allowed to flow through a stalagmometer into olive oil is found to vary in a remarkable manner with variations in the proportions of $\mathrm{NaCl}$ and $\mathrm{CaCl}_{2}$ in the solution; thus with an alkalinity of .ooIn $\mathrm{NaOH}$, the following number of drops per minute were observed with different solutions:

\section{Solution}

Control (no salt)...............

Pure $0.15 \mathrm{~m} \mathrm{NaCl} \ldots \ldots \ldots \ldots . .300$

Pure $0.0015 \mathrm{~m} \mathrm{CaCl}_{2} \ldots \ldots \ldots \ldots .24$

$0.15 \mathrm{~m} \mathrm{NaCl}+0.0015 \mathrm{~m} \mathrm{CaCl}_{2} \ldots . \quad 44$

$0.3 \mathrm{~m} \mathrm{NaCl}+0.003 \mathrm{~m} \mathrm{CaCl}_{2}: \ldots \ldots \quad 43$

$0.45 \mathrm{~m} \mathrm{NaCl}+0.005 \mathrm{~m} \mathrm{CaCl}_{2} \ldots \ldots .43$

$0.6 \mathrm{~m} \mathrm{NaCl}+0.0 \mathrm{I} \mathrm{m} \mathrm{CaCl}_{2} \ldots \ldots . \quad 43$

The pure $\mathrm{NaCl}$ solution greatly promotes the tendency toward fine subdivision of the drops; the $\mathrm{CaCl}_{2}$ decreases this tendency; at a certain ratio of the two salts (Na: Ca about 50 or 100:I) the two tendencies counteract each other. The result depends on the

${ }^{\mathrm{I}}$ Clowes, Journal of Physical Chemistry, $\mathrm{XX}$ (1916), 407. 
different solubilities of the Na-oleate and the Ca-oleate in the two phases, the former being soluble in water but not in oil, the latter soluble in oil but not in water. The surface-tension conditions at the interface are accordingly oppositely affected by the two salts, with correspondingly opposite effects on the state of dispersion; at a certain ratio of concentrations the two opposite effects are balanced. Clowes shows that when equal volumes of oil and alkaline salt solution (mixtures of $\mathrm{n}$ / Io $\mathrm{NaOH}$ and $\mathrm{n}$ / o $\mathrm{CaCl}_{2}$ ) are shaken together, the effect varies according to the proportions of $\mathrm{Na}$ and $\mathrm{Ca}$ in the solution; when $\mathrm{Na}$ is present in excess, the oil is dispersed as droplets in a continuous water phase, while when $\mathrm{Ca}$ is in excess the water is dispersed as droplets in a continuous oil phase. A reversal of phase-relations may thus be accomplished in an emulsion system by changing the salt-content; and this conclusion has highly important biological applications, since it bears closely on the problem of the relations between the lipoid and the aqueous components in the living protoplasmic system. Any system in which the oil (organic solvent or lipoid) phase is the continuous one is permeable to oil-soluble substances, but not to water-soluble substances which are oil-insoluble; and the general correspondence of this condition with that observed in living protoplasm suggests the possibility that the external layer of the living plasma membrane consists (at least during the greater part of its existence) of a continuous layer of lipoid material, the continuous condition depending on the presence of compounds with properties like those of $\mathrm{Ca}$ soaps. The importance of $\mathrm{Ca}$ to the semi-permeability and water-resisting properties 
of the protoplasmic surface-film, on this hypothesis, is evident.

The possibility of a reversibility of phase-relations under salt action ${ }^{x}$ implies the possibility of inducing reversible changes of permeability under these conditions, and such reversible changes are, as we have seen, a characteristic feature of the living plasma membranes. Clowes has in fact constructed a model in which an emulsion consisting of equal volumes of oil and a mixed salt solution (containing $\mathrm{NaCl}, \mathrm{KCl}$, and $\mathrm{CaCl}_{2}$ in proportions similar to those of sea water and slightly alkaline) is supported in the interstices of a paper partition (sheets of filter paper) fixed in position by rings of rubber in the interior of a U-tube. ${ }^{2}$ The electrical conductivity of the emulsion-permeated paper is then found to vary, when the solution in contact with it is changed, in a manner resembling in general that shown by the partition of living plant-tissue in Osterhout's experiments. In pure $\mathrm{NaCl}$ solution the conductivity is rapidly increased; in pure $\mathrm{CaCl}_{2}$ it is decreased; and the changes of conductivity are reversible if they are not allowed to proceed too far. It is assumed that in the capillary interstices the emulsion undergoes reversible changes of phase of the above-described kind, the conversion of the

${ }^{x}$ For the conditions of the reversibility of phase-relations in emulsions cf. Newman, Journal of Physical Chemistry, XVIII (1914), 34; Briggs and Schmidt, ibid., XIX (1915), 478; Clowes, ibid., XX (1916), 407; Bhatnagar, Jour. Chem. Soc. Trans., CXVII (1920), 542; also Physics and Chemistry of Colloids, Discussion of Faraday Society and Physical Society of London (192I), p. 27; also in Kolloid-Z., XiviII (I92I), 206.

${ }^{2}$ Clowes, Proceedings of the Sociely of Expcrimental Biology and Medicine, XV (IgI8), I08. 
oil from the continuous to the discontinuous phase corresponding to an increase in conductivity and vice versa.

It will be remembered that water-insoluble colloidal material $\left(\mathrm{Cu}_{2} \mathrm{FeCy}_{6}\right)$ held in the interstices of a supporting structure forms the essential composition of the semipermeable membranes used in the osmotic pressure determinations of Pfeffer and Morse; in such membranes, also, permeability is changed by the action of salts. The conditions in living membranes, while probably similar, in the above broad sense, to those in such structurally composite membranes, are vastly more complex, chiefly on account of the constant presence of the metabolic factor. Clowes propounds the general hypothesis that "variations in the permeability of the protoplasmic membranes are attributable to the action of electrolytes and metabolic products on delicately balanced interfacial soap-films and emulsion systems, and that proteins may play no part in the valve-like mechanism controlling permeability other than to afford a supporting filamentous or mesh-like structure."x

Such an arrangement need not be taken too literally as an exact model of the conditions in the protoplasmic surface-films. It seems probable, however, that in the plasma membrane there is a combination of a relatively permanent supporting structure, presumably protein, with a variable emulsion-like component whose waterinsoluble phase is normally so disposed as to block, with a considerable degree of completeness, the interstitial spaces or capillary channels of the membrane. Variability in this emulsion, under the influence of salt solu-

${ }^{s}$ Clowes, op. cit., p. IIo. 
tions or other factors, involves variability in the permeability of the partition; and as a secondary consequence other conditions depending on that permeability, such as electrical polarization, are affected. Such a conception implies that changes in the supporting protcin structure may also influence permeability; but it regards the normal or physiological variations in this property as dependent chiefly on variations in the state of the most external water-insoluble or lipoid portion of the protoplasmic complex. Such a conception allows for a wide range of variability in the permeability of the membrane. The latter is not to be regarded as a continuous lipoid sheet under one set of conditions, which changes without transition, under other conditions, in to a state in which the lipoid becomes the discontinuous and the aqueous phase the continuous phase. A balanced state, with fluctuations on either side of a mean, which is regulatively maintained by metabolic processes, is rather the one to be conceived as representing the condition actually existing during life.

A simple case of salt-antagonism, which I have recently studied in the starfish egg, ${ }^{\mathrm{r}}$ appears to throw light upon the more specifically chemical conditions of these phenomena. The fully mature starfish egg ( $c a$. I60 $\mu$ in diameter) is surrounded with a layer of jelly-like substance, of I5 to $20 \mu$ in diameter, consisting of water-swollen material (of undetermined nature) separated or secreted from the egg-protoplasm. This layer is rendered visible by mounting the eggs on a slide, with cover-glass, in a suspension of India ink, to which the jelly is impermeable; it then appears under the micros Jour. Gen. Physiol., III (I92I), 783 . 
scope as a clear halo surrounding each egg. When the egg is washed in pure isotonic $\mathrm{NaCl}$ solution ( $0.54 \mathrm{~m}$ ), preferably with the aid of centrifuging, the jelly swells and dissolves; but in $\mathrm{NaCl}$ solution containing a little $\mathrm{CaCl}_{2}\left(\frac{\mathrm{m}}{400}\right.$ is sufficient) it remains intact. Salts of several other metals ( $\mathrm{Mg}, \mathrm{Mn}, \mathrm{Co}, \mathrm{Ni}, \mathrm{Al}$ ) have been found to have a similar effect. ${ }^{x}$ The jelly layer, in the presence of the salts of sea water and in mixtures of $\mathrm{NaCl}$ and $\mathrm{CaCl}_{2}$, thus possesses a certain waterinsolubility and physical consistency; these properties are apparently dependent on the presence of a calcium compound (possibly proteinate) with definite physical properties (especially water-insolubility and lack of tendency to swell) which preserves the characteristic structure and consistency of the whole layer. In pure $\mathrm{NaCl}$ solution this constituent is replaced by the corresponding sodium compound which is water-soluble and swells readily, hence the coherence and insolubility of the layer as a whole are lost.

This process may be regarded as a model of the essential kind of change occurring in the plasma membranes of living cells in pure $\mathrm{NaCl}$ solution. The surface of the starfish egg is in fact physically altered by $\mathrm{NaCl}$ solution in a characteristic manner; unfertilized eggs placed in the pure solution cohere in clumps or agglutinate; many eggs also form fertilization-membranes (when returned to sea water) and show evidence of partial activation by cleaving and in some cases developing to the blastula stage. All of these effects of the pure

${ }^{\mathrm{r}}$ Unpublished experiments in the Nela Research Laboratory at the Marine Biological Laboratory at Woods Hole. 
solution are prevented in the presence of a little $\mathrm{CaCl}_{2}$. The toxic action of the pure solution and also its membrane-forming action are correlated with a permeability-increasing action, as in the other cases cited above; and the correspondence with the behavior of the jelly seems to imply that the increase of permeability in the pure solution is to be referred also to the replacement of water-insoluble $\mathrm{Ca}$ compounds (e.g., $\mathrm{Ca}$ proteinates or soaps), on which the properties of the plasma membrane depend, by soluble Na compounds.

There is much independent evidence that this rôle of calcium compounds-i.e., of determining the properties of the surface layers of cells-is a general one; we may thus understand the importance of $\mathrm{Ca}$ to all normal cell-processes (stimulation, etc.) which depend on changes in the surface layers. The peculiar relation of $\mathrm{Ca}$ to the coherence of blastomeres and of plant cells has already been mentioned; according to the earlier work of Mangin, Ca compounds ("Ca-pectate") in the middle lamella of plant cells are essential to the structural coherence of cellular tissues; when the $\mathrm{Ca}$ is replaced by $\mathrm{Na}$, the cells tend to fall apart. Similar phenomena are also well known in the epithelial tissues of animals; e.g., in the ciliated epithelium of Mitylus the cells swell and fall apart in pure solutions of many $\mathrm{Na}$ and $\mathrm{K}$ salts, and this effect is prevented by Ca. ${ }^{\mathrm{I}}$ Recently the changes occurring in plant tissues in pure $\mathrm{NaCl}$ solutions have been studied in much detail by Hansteen. ${ }^{2}$ The

${ }^{\prime}$ R. S. Lillie, American Journal of Physiology, XVII (Igo6), S9; cf. P. I 22 .

${ }^{2}$ Hansteen (Hansteen-Cranner), Jahrb. wiss. Bolanik, XI,III (1910), 374; LIII (19I3-I4), 536; Ber. deutsch. Botan. Ges., XXXVII (I919), 380. Also his recent book, Zur Biochemie u. Physiologie der Grenzschichten lebender Pfanzenzellen, Kristiania (I922). 
general effects are swelling, loss of consistency or turgor, and disintegration of the tissue, accompanied by loosening of intercellular coherence; these effects are all prevented or greatly decreased in the presence of a little $\mathrm{Ca}$. Hansteen believes that water-insoluble $\mathrm{Ca}$ compounds are essential constituents of living protoplasm in general, and that they are present especially in the protoplasmic surface layers and other solid structures; he also cites evidence indicating that these compounds are lipoid in nature. According to his conception, pure alkali salt solutions attack the surface layers of cells because they alter the water-insoluble Ca-lipoid compounds there present, converting them into watersoluble compounds and secondarily inducing absorption of water and structural breakdown. Such effects are apparently of the same nature as the disintegration of the cilia of marine animals (Arenicola) and the general loss of semi-permeability of plant and animal cells in pure salt solutions, already described. They are consistent with the general view that in the formation of the solid or permanent (water-insoluble) protoplasmic structures, $\mathrm{Ca}$ compounds play an essential part. Hansteen found that the presence of more than the normal concentration of $\mathrm{Ca}$ salts in culture solutions favored profuse branching and the formation of an abundance of root hairs in seedlings; Wiechmann, in Höber's laboratory, has recently confirmed this result, and has found further that $\mathrm{Sr}, \mathrm{Ba}$, and a few heavy metals $(\mathrm{Mn}, \mathrm{Ni}, \mathrm{Co}$ ) act similarly to $\mathrm{Ca}$, while $\mathrm{Mg}$ is ineffective. ${ }^{\mathrm{I}}$ It would seem that certain necessary physical properties of protoplasmic structures, such as rigidity, water-

${ }^{x}$ Wiechmann, Arch. ges. Physiol., CLXXXII (1920), 99. 
insolubility, and impermeability to water-soluble substances (like sugar and salts), require the presence of Ca compounds. These compounds impart the necessary structural stability to the whole protoplasmic complex. A fact of interest in relation to this question is Meigs's recent observation that an approach to semi-permeability can be imparted to certain artificial colloidal membranes, otherwise highly permeable, by depositing insoluble $\mathrm{Ca}$ salts (phosphate) in their substance. ${ }^{x}$

These facts and considerations are consistent with the view that $\mathrm{Ca}$ compounds, e.g., $\mathrm{Ca}$ soaps, play a similar part in the surface layers of protoplasm, and the recent interesting experiments of Clark ${ }^{2}$ with the frog's heart lend support to this general conception. He finds that the heart, after being weakened by prolonged perfusion with Ringer's solution, rapidly regains its vigor if perfused with Ringer's solution to which serum, serum-lipoids, lecithin, or soaps of higher fatty acids, have been added. During perfusion with pure Ringer's solution the heart loses to the solution some material which has a similar reviving action when perfused through other exhausted hearts. In order that these substances, or soap, should exhibit this beneficial action, calcium must be present. He concludes that the beneficial action of the soaps is associated with the adsorption of a water-soluble Ca soap or similar compound upon the surface of the muscle cells, and puts forward the hypothesis "that the activity of the heart is dependent upon the semi-permeability of the cell to electrolytes, that this is

'Meigs, American Journal of Physiology, XXXVIII (19r5), 456.

${ }^{2}$ A. J. Clark, Journal of Physiology, XLVII (r9r3), 66; also Clark and Daly, ibid., LIV (I921), 367. 
dependent on the presence of $\mathrm{Ca}$ and lipoids at the surface of the cells, and that during perfusion the heart loses lipoids and becomes more permeable to electrolytes." This conception of semi-permeability as dependent on the presence of lipoids, and of the state of the lipoids as determined by the salts of the medium, is in harmony with much recent work. ${ }^{I}$ It also affords a point of view from which it is possible to understand why the physiological effects of salts and of lipoidsolvent compounds should have so much in common.

r Blackman, "The Plasmatic Membrane and Its Organization," New Phytologist, XI (I9I2), I80; Clowes, loc. cit; Czapek, Oberfächenspannung der Plasmahaut, Jena (I9II); McDougall, Science, LV (I922), 653; Hansteen-Cranner, loc cit. See also Stiles's review of the subject of cell permeability in New Phytologist, XX, XXI, XXII (1921-23). 


\section{CHAPTER IX}

GENERAL PHYSIOLOGICAL ACTION OF LIPOID-

ALTERANT AND SURFACE-ACTIVE SUBSTANCES

From the physiological point of view the recersible forms of salt action are the important ones; the properties and activities of living protoplasm may thus be modified by changing the salt-content of the medium, and return to the normal when the original salt-content is restored. Such reversible effects are of special biological interest, since their essential conditions are in all likelihood similar to those controlling the normal variations of activity. Substances are continually being formed in metabolism (e.g., $\mathrm{CO}_{2}$ and other acids) which directly influence protoplasmic action. It is, therefore, of fundamental interest to note the existence of another large class of substances, many of which are chemically indifferent, i.e., not readily oxidized or reduced (hydrocarbons and their substitution-products), which have a profound influence on protoplasm, completely reversible within wide limits. These substances are those organic compounds, varying widely in their chemical nature, which have in common two general physical properties: (I) a solvent action on, or solubility in, the water-insoluble organic constituents of protoplasm (fats, lipoids, etc.); and (2) a high degree of surface-activity, i.e., influence on the surface-tension at the boundary between water and non-aqueous phases. These compounds appear also to produce their physiological effects by altering the 
structural substratum of protoplasm in a manner which does not permanently change its properties or physical state. Hence in their presence physiological processes are modified temporarily in rate or character, and resume their former conditions when the substance is removed. The special affinity of these compounds for substances having fat-like properties indicates that their primary action is on the lipoid constituents of protoplasm; their possible action on proteins, however, is also to be considered. In general they include the substances comprised in Overton's first group (alcohols, ethers, esters, normal and substituted hydrocarbons, etc.).

We distinguish, therefore, two chief groups of compounds which by means of their reversible influence on the structural substratum of protoplasm may modify vital processes without affecting them permanently or injuriously: (a) neutral salts or other electrolytes (acid and alkali), and (b) lipoid-solvent or surface-active organic compounds. These two groups may be characterized respectively as general colloid-alterants and lipoid-alterants. The compounds of these groups differ somewhat sharply in their physiological action from those compounds whose chemical effects tend to be irreversible; the latter include most of the strong oxidizing and reducing agents and the salts of heavy metals; usually these are not capable of modifying physiological processes without permanent injury; hence they are toxic or poisonous in small doses. Recovery from the effects of this "poisonous" group depends upon the reparative activity of the living protoplasm, just as does recovery from mechanical injury, and not upon a simple reversal of the chemical or other action of the compound. 
The most remarkable general physiological effect produced by the lipoid-alterant substances is a reversible suppression of irritability or spontaneous activity. This effect always appears in certain definite, not too high, concentrations of these compounds, and constitutes the phenomenon of narcosis or anæsthesia, which is universal in living matter. ${ }^{\mathrm{I}}$ The power of inducing this state seems to be independent of the special chemical nature of the narcotizing compound; evidently this power is connected in some manner with the general physical properties just named; and the question first arises whether the lipoid-solubility of these compounds or their surface-activity is the property primarily responsible for this characteristic action.

\section{CORRELATION BETWEEN PHYSIOLOGICAL ACTION AND} LIPOID-SOLUBILITY

The existence of a relation between the solubility of chemical compounds in fats and their narcotic action was early noted, first by Bibra and Harless in I $\$_{47}$, and later by Claude Bernard, Hermann, Richet, Ehrlich, and others. ${ }^{2}$ Richet propounded the rule that any narcotizing compound has the stronger action as a narcotic the lower its solubility in water. This is similar to the rule of Overton and Meyer that the narcotic effectiveness of a compound runs parallel with

I"We may say that everything living is sensitive and can be anæsthetized; whatever is not sensitive is not living and cannot be anæsthetized."-Claude Bernard, address, "La Sensibilité" (delivered in 1876), published in his book, La Science Expérimentale, Paris (1Sgo).

${ }^{2}$ Cf. Overton, Studien über die Narkose, Jena (Igor), for a historical account of the earlier work on narcosis. 
its oil-water partition-coefficient, a relation first studied systematically by these investigators. ${ }^{x}$

In his investigations on permeability, Overton had reached the conclusion that solubility in lipoids was the chief factor determining the entrance of compounds into cells; such readily penetrating compounds belong for the most part to the narcotizing group; and a detailed study of the phenomena of narcosis in tadpoles demonstrated a close parallelism between the relative solubilities of a large number of organic compounds in oil and water and their narcotizing action. ${ }^{2}$ The ratio according to which any compound is distributed between these solvents (when the solvents are in contact and equilibrium is reached) is a measure of its relative solubility in the two; this ratio is known as the "partitioncoefficient." In the early members of any homologous series of compounds the ratio of oil-solubility to watersolubility increases progressively as the molecular weight increases, and the same is true of the narcotizing properties of the compounds. For example, with the ethyl esters of the first five fatty acids, Overton found the concentrations required for the complete narcosis of tadpoles to be as indicated in the table (p. I9I).

The narcotic effectiveness of the ester increases regularly as its water-solubility decreases; and in general each member of the series is from two to three times as effective as its immediate predecessor. Relations of a similar kind were found with other series,

${ }^{s}$ Overton, Vierteljahrschriften d. Naturf. Gesellschaft, Zürich, XLIV (1899), 88; Studien über die Narkose (1901); H. H. Meyer, Arch.f. exper. Path. u. Pharmakol., XLII (I899), Iо9.

${ }^{2}$ Studien über die Narkose. 
including hydrocarbons, alcohols, aldehydes, ketones, ethers, and various substituted compounds. Any increase in the oil-water partition-coefficient was associated with increase in narcotic effectiveness. Overton accordingly drew the conclusion that the narcotics act by dissolving in certain oil-like or fatty constituents of the irritable cells (in this case nerve cells); and he identified these constituents with the lipoids, especially lecithin and cholesterol, which appear to be always present in protoplasm. The essential determining condition of anæsthesia, according to his view, is the solution

\section{Ester}

Ethyl formate .....

Ethyl acetate......

Ethyl propionate...

Ethyl butyrate....

Ethyl valerianate...
Narcotizing Concentrations (Mols. per litre)

.07 to $.09 \mathrm{~m}$

$.03 \mathrm{~m}$

.OI to .OI $2 \mathrm{~m}$

$.0043 \mathrm{~m}$

$.0019 \mathrm{~m}$

\section{Solubilities in Oil} and IVater

Oil: water 4 : $\mathrm{I}$

In 15.2 parts water; in all parts oil

In 50 parts water; in all parts oil

In I90 parts water; in all parts oil

In 500 parts water; in all parts oil

of the narcotic compound in these cell constituents; when the lipoids are charged or impregnated with the compound, they undergo a change of physical properties, entailing corresponding alterations in the irritability of the cell. Meyer drew independently a similar conclusion; ${ }^{\mathrm{r}}$ he pointed out that the narcotizability of cells seems to be related to the nature and proportion of the lipoids present in the protoplasm; e.g., the high susceptibility of nerve cells to narcosis is a correlative of their high lipoid-content. Different narcotics act unequally because they are distributed in unequal ratios between the

'Meyer, loc. cit. 
lipoid and the aqueous phases of protoplasm; in general, the greater the relative lipoid-solubility, the larger the proportion of the anæsthetic compound which is in solution in the lipoids when the partition-equilibrium is reached. Hence when a compound has a very high lipoid-solubility it may exert narcotic action in extremely dilute solution; phenanthrene, for example, was found by Overton to narcotize tadpoles in dilutions of one part in $\mathrm{I}, 500,000$ of water.

The general conception known as the "OvertonMeyer theory of narcosis" may be defined as follows. The solubility of narcotizing compounds in the celllipoids forms the basis of their narcotic and presumably other pharmacological properties. By dissolving in the lipoids, such compounds alter the physical properties of these essential components of the protoplasmic system, and hence all properties of the system, especially irritability, which are dependent on the state of the lipoids. Since simple solution without chemical combination is the basis of this effect, the latter is readily reversed by allowing the compounds to diffuse away.

The general conclusion that selective solubility is the essential basis of narcotic action does not, however, necessarily follow, since the same reasoning would apply to other physical effects which are reversible under similar conditions; e.g., effects dependent on surfaceactivity, involving a lowering of surface-tension at the protoplasmic phase-boundaries and a concentration of the narcotizing compound at these surfaces. Probably, however, the case is not one of alternatives; if lipoids are present in the system, any substances which are soluble in these components must inevitably dissolve 
according to the partition-ratios. Any tendency to concentrate at surfaces (e.g., the general cell surface or the surfaces of other lipoid-containing protoplasmic structures) would be favorable to such solution; i.e., would render it more rapid and greater in degree than it would be otherwise, since the partition-equilibrium would then be between the solution in contact with the surface of the lipoid particle and the solution in the interior of the particle.

Meyer's experiments on the influence of temperature on the critical anæsthetizing concentrations of certain compounds $^{\mathrm{x}}$ gave further indications that the solubility of these compounds in the cell-lipoids is the essential factor in the physiological effect. He chose six compounds whose oil-water partition-ratios vary considerably with temperature, and determined the minimal concentrations required to anæsthetize tadpoles at the two temperatures $3^{\circ}$ and $30^{\circ}$. These concentrations are given in the following table:

Anæsthetic

Critical Concentration for Anæsthesia At $3^{\circ}$

(A) Salicylamide....m m/ I 300

Benzamide....... m/ $/ 500$

Monoacetin...... m/9o

(B) Ethyl Alcohol.... m/3

Chloral Hydrate... m/50 Acetone........m m/3
At $30^{\circ}$

$\mathrm{m} / 600$

$\mathrm{m} / 200$

$\mathrm{m} / 70$

$\mathrm{m} / 7$

$\mathrm{m} / 250$

$\mathrm{m} / 7$
Oil-Water PartitionCoefficients

$$
\text { At } 3^{\circ}
$$

At $30^{\circ}$

22. 23

I 4

0.67

0.43

0.099

0.066

0.026

0.247

0.053

0. 236

o. 146

In the first three compounds (A) the relative lipoidsolubility decreases with rise of temperature, and the

${ }^{3}$ Meyer, Arch. f. exper. Path. u. Pharmakol., XLVI (Igor), 33s. Recently Mary E. Collett has investigated the effects of variation of temperature on the narcotic action of various compounds on marine organisms; cf. Proceedings of the Sociely for Experimental Biology and Medicine, XX (I923), 259. 
critical narcotizing concentration increases; in the last three (B) the conditions are reversed. Tadpoles completely anæsthetized at $30^{\circ}$ in $\mathrm{m} / 250$ chloral hydrate revive and become active on cooling the solution; on warming they again become inactive. Such an effect is difficult to explain except on the basis of the greater lipoid-solubility of the anæsthetic at the higher temperature, since adsorption is in general increased by lowering the temperature. Meyer therefore concludes that the anæsthetic produces its effect by dissolving in the celllipoids. Such experiments seem to indicate clearly that the solvent action of the protoplasmic lipoids is a main factor in anæsthesia; and since anæsthesia has close affinities with normal variations of irritability, such as sleep and fatigue, they also point to the conclusion that under normal conditions the lipoids are important in the cell largely because of their peculiar properties as solvents.

The relation of the solvent properties of lipoids to permeability has already been considered. The lipoids thus represent the organic solvents of the cell, and apparently the properties of the protoplasmic system vary according to the nature and concentration of the substances which they hold in solution. This is a conclusion of much general interest, apart from its special relation to anæsthesia, since variations in the proportions of water-soluble to lipoid-soluble substances no doubt occur constantly in living protoplasm. The question of why such variations alter irritability, spontaneous activity, and metabolism will be considered more fully later in connection with stimulation.

In many cases it has been shown that the organic anæsthetics accumulate in cells in greater concentration 
than in the medium, and this fact is usually interpreted as favoring the partition theory of narcosis. Pohl ${ }^{x}$ found that the blood corpuscles and brain of decply narcotized dogs contained from three to four times as much chloroform as the serum; Hedin ${ }^{2}$ investigated cryoscopically the distribution of alcohols, aldehydes, ketones, esters, and ether between corpuscles and plasma, and found that most compounds, especially the more highly lipoid-soluble, collected in higher concentration in the cells than in the plasma. More recently Warburg and Wiesel ${ }^{3}$ have made similar observations on the blood corpuscles of birds, using alcohols, ketones, urethanes, thymol, formaldehyde, and HCN. All of these compounds, in appropriate concentrations, were found to decrease oxygen consumption, and the tendency to concentrate in the cells ran closely parallel with this effect. Thus the lower alcohols (up to butyl alcohol) were the least effective in reducing oxygen consumption, and showed correspondingly a somewhat lower solubility in the protoplasm than in the medium; methyl urethane, diethyl urea, and acetone behaved similarly: amyl alcohol and isobutyl urethane were more effective, and were about equally distributed between cells and medium; while the most effective substances-phenyl-methyl ketone, thymol, phenyl urethane-all showed decided concentration in the cells. When solutions were used that decreased the oxygen consumption by 50 per cent, phenyl-methyl ketone was found to be about twice, phenyl urethane three times, and thymol nine times as

I Pohl, Arch. exper. Path. u. Pharmakol., XXVIII (ISgr), 239.

${ }^{2}$ Hedin, Arch. ges. Physiol., LXVIII (1897), 229.

3 Warburg and Wiesel, Arch. ges. Physiol., CXLIV (rgr2), 465. 
concentrated in the cells as in the medium. Formaldehyde and HCN also underwent concentration in the cells. The series, methyl alcohol $<$ butyl alcohol $<$ amyl alcohol $<$ phenyl-methyl ketone $<$ phenyl urethane $<$ thymol represents the order both of increasing physiological action and of increasing concentration in the cells.

On the whole the foregoing studies of the distribution of narcotics between cell and medium appear to favor the partition theory of the action of these compounds. It should be pointed out, however, that the order of relative adsorption is in general the same as that of lipoidsolubility. The probability, as already pointed out, is that both solution and adsorption are factors in the total effect.

\section{CORRELATION BETWEEN PHYSIOLOGICAL ACTION}

\section{AND SURFACE-ACTIVITY}

The relation between the narcotic or other physiological action of organic compounds and their influence on surface-conditions has recently received much investigation. The majority of compounds of the lipoidalterant group have a marked influence in lowering the surface-tension at the boundary-surfaces between their aqueous solutions and air or other adjoining phase; at the same time, in accordance with the Gibbs-Thomson rule, they undergo increase of concentration (or adsorption) at such surfaces. In homologous series both the influence on surface-tension and the degree of adsorption increase progressively with increase in molecular weight; and the view that the physiological action is determined by this surface-action, i.e., by the condensation of the compounds at the protoplasmic phase-boundaries, rather 
than by solution in the lipoids has been supported by many recent investigators, especially Czapek, 'Traube, and Warburg. ${ }^{\mathrm{I}}$

The general fact that the physiological action of homologous compounds increases progressively with increase in molecular weight has long been noted. Richardson, ${ }^{2}$ in 1869 , in a study of the pharmacological action of alcohols, called attention to this rule, which applies also to the effects on lower organisms; thus, according to Regnard, ${ }^{3}$ the first six alcohols have equal effects in suppressing the growth of yeast in the following concentrations:

Alcohol

$\mathrm{CH}_{3} \mathrm{OH} \ldots \ldots \ldots \ldots \ldots \ldots$

$\mathrm{C}_{2} \mathrm{H}_{5} \mathrm{OH}$

$\mathrm{C}_{3} \mathrm{H}_{7} \mathrm{OH}$

$\mathrm{C}_{4} \mathrm{H}_{9} \mathrm{OH}$

$\mathrm{C}_{5} \mathrm{H}_{\mathrm{II}} \mathrm{OH}$

$\mathrm{C}_{6} \mathrm{H}_{13} \mathrm{OH}$...
Volumes (per cent)

20

15

IO

$2 \cdot 5$

I

0. 2

In a series of papers beginning in I904, Traube has directed special attention to the parallelism between surface-activity and physiological action. ${ }^{4}$ For example,

I Czapek, Oberfä̈chenspannung der Plasmahaul, Jena (19I I); Traube, "Theorie der Narkose," Arch. ges. Physiol., CLIII (19I3), 276; CLA (I9I5), 50I. Cf. also "Theorie des Haftdrucks und Lipoidtheoric," Biochem. Z., LIV (I9I3), 305, and other papers there cited. Warburg, see below.

${ }^{2}$ Richardson, "Physiological Researches on Alcohols," Medical Times and Gazette, VIII (I869) (cited from Czapek, loc. cil.).

${ }^{3}$ Regnard, Compt. rend. Soc. Biol., X (ISSg), I24. Warburg and Wiesel obtained similar results with the series of urethanes (loc. cit.).

${ }_{4}^{4}$ Traube, Arch. ges. Physiol., CV (1904), 541, 559; further references in the papers cited above. 
the surface-tensions (against air) of $0.25 \mathrm{~m}$ aqueous solutions of alcohols decrease in regular order as follows:

\begin{tabular}{|c|c|}
\hline Alcohol & $\begin{array}{l}\text { Surface-Ten } \\
\text { (Mgms. per }\end{array}$ \\
\hline $\mathrm{CH}_{3} \mathrm{OH}$. & 7.05 \\
\hline $\mathrm{C}_{2} \mathrm{H}_{5} \mathrm{OH}$. . & 6.73 \\
\hline $\mathrm{C}_{3} \mathrm{H}_{7} \mathrm{OH}$. & 2.89 \\
\hline $\mathrm{C}_{4} \mathrm{H}_{9} \mathrm{OH}$. & 4.49 \\
\hline $\mathrm{C}_{5} \mathrm{H}_{\mathrm{II}} \mathrm{OH}$. & 3.05 \\
\hline$\left(\mathrm{H}_{2} \mathrm{O}\right) \ldots$ & \\
\hline
\end{tabular}

The order of relative adsorption by charcoal and other adsorbents is similar; if the degree of adsorption runs parallel with the lowering effect on surface-tension, and if equal adsorption corresponds to equal physiological action, we should expect that solutions of the same surface-tension (isocapillary solutions) would have the same physiological effect. Czapek and Traube have in fact demonstrated a close parallelism between the influence of a large number of compounds on air-water surfacetension and their physiological action. Traube has called attention to the fact that in a homologous series of compounds the degree of activity, both physical and physiological, increases very generally about three times with each increase in molecular weight. According to this rule the isocapillary concentrations of the successive compounds of the series should diminish in geometrical progression, with one-third as exponent, as the molecular weight increases. He gives the following determinations for the series of alkyl acetates:

Ester and Concentration

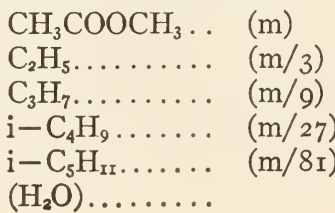

Capillary Height

58 . I m

58.0

57.7

58.8

59.9

(9I.5) 
In a considerable number of cases the degree of physiological action has been shown to follow a similar rule; the observations of Fühner on hrmolysis (cited below) are a good example.

If physiological activity is in fact a function of capillary activity, solutions of equal surface-tension should exhibit equal narcotic action or otherwise produce equal effects in protoplasm; and Czapek has brought forward evidence that this is very frequently the case. ${ }^{x}$ Using a large number of surface-active organic compounds, he determined the surface-tensions of those solutions which had equal effect in liberating tannin from plant cells (chiefly the leaves of Echeveria); this effect depends on a permeability-increasing action analogous to that accompanying cytolysis. In general he finds that solutions of a concentration just sufficient to cause exosmosis of tannin have very nearly the same surface-tension against air; viz., about two-thirds that of pure water; according to his hypothesis, the surface-tension of the protoplasm becomes zero in such solutions and an effective surface of separation ceases to exist. Kisch ${ }^{2}$ also finds that isocapillary solutions of alcohols have equal effects in liberating invertase from yeast and molds and in inhibiting the growth of yeast cells; and $H$. Zuckerkandl ${ }^{3}$ has observed a similar relation in the protoplasmic streaming of plant cells. According to Traube and others, hæmolysis follows the same rule.4

${ }^{I}$ Czapek, loc. cit.

${ }^{2}$ Kisch, Biochem. Zeitschrift, XL (IgI2), I52.

3 H. Nothmann-Zuckerkandl, Biochem. Zeitschrijl, XLV (1912), 412 .

${ }^{4}$ Traube, loc. cil.; Fühner and Neubaucr, Zentralbl. f. Physiol., XX (Ig06), II7; Arch. exper. Path. u. Pharmakol., LV'I (1907), 333. 
The following table summarizes some of the results of Czapek and Kisch with alcohols.

\begin{tabular}{|c|c|c|}
\hline \multirow[b]{2}{*}{ ALсоног } & \multicolumn{2}{|c|}{ CRITICAL SURFACE-TENSIONS (WATER $=\mathrm{I}$ ) OF SOLUTIONS } \\
\hline & $\begin{array}{l}\text { A. Exosmosis of Tannin from } \\
\text { Cells of Echeveria }\end{array}$ & $\begin{array}{l}\text { B. Inhibition of } \\
\text { Growth of Yeast }\end{array}$ \\
\hline Methyl..... & 0.7 & $0.5^{\mathrm{I}}$ \\
\hline Ethyl...... & 0.67 & 0.48 \\
\hline N-propyl.... & 0.675 & ca. 0.49 \\
\hline I-propyl..... & 0.69 & $\ldots \ldots$ \\
\hline N-butyl.... & 0.69 & $\cdots$ \\
\hline I-butyl...... & $0.66_{5}$ & $c a .0 .5$ \\
\hline I-amyl..... & 0.665 & 0.49 \\
\hline
\end{tabular}

Czapek's ascription of the effects which he observes to a definite or critical lowering of the surface-tension of the plasma membrane is, however, of doubtful validity, since there is no necessary parallelism between the influence of a given substance on the surface-tension at a water-air interface and its influence on the tensions at other interfaces. This has recently been pointed out by Lorant; ${ }^{\prime}$ for example, in comparing the tensions exhibited by various liquids in contact with air and with water, respectively, Lorant finds the following:

S.T. of $\mathrm{CHCl}_{3} /$ water is about $6 \%-4 \%>$ S.T. of $\mathrm{CHCl}_{3} /$ air

S.T. of $\mathrm{CCl}_{4} /$ water is about $65 \%>$ S.T. of $\mathrm{CCl}_{4} /$ air

S.T. of $\mathrm{C}_{6} \mathrm{H}_{6} /$ water is about $33 \%>$ S.T. of $\mathrm{C}_{6} \mathrm{H}_{6} /$ air

S.T. of $\mathrm{C}_{6} \mathrm{H}_{5} \mathrm{NO}_{2} /$ water is about $4 \mathrm{I}-42 \%<$ S.T. of $\mathrm{C}_{6} \mathrm{H}_{5} \mathrm{NO}_{2} /$ air S.T. of $\mathrm{C}_{6} \mathrm{H}_{\mathrm{II}} \mathrm{OH} /$ water is about $77 \%<\mathrm{S}$.T. of $\mathrm{C}_{6} \mathrm{H}_{\mathrm{II}} \mathrm{OH} /$ air

Lorant also made observations on the surfacetensions between various organic fluids (e.g., ether) and salt solutions. Usually the influence of neutral salts on surface-tension was in the direction of an increase. Of the different anions $\mathrm{Cl}$ has the greatest effect, and I and

${ }^{x}$ Lorant, Arch. ges. Physiol., CLVII (Igr4), 2 Ir. 
CNS the least; with ethyl ether and nitromethane, the chlorides, sulphates, and bromides increased the interfacial tension, while the iodides and thiocyanates decreased it. Similar conditions were found with $\mathrm{CHCl}_{3}$ and $\mathrm{CCl}_{4}$, but in this case iodide also somewhat increased the surface-tension.

It would appear that the physical relations (of adhesion, mutual solubility, etc.) between water and the organic compound, as well as between the latter and the non-aqueous protoplasmic phase or structure (c.g.. membrane) are of importance in the physiological effect. According to Traube the narcotic action of organic compounds is determined by what he calls their "Haftdruck" ("adhesion-tension"), i.e., special attraction to or affinity for water; ${ }^{\mathrm{I}}$ the tendency of any compound to pass out of aqueous solution and concentrate in the surface layer between water and the other phase-i.e., to undergo adsorption-is in general the greater the less its affinity for water. This is one manner of interpreting the relation noted by Richet and others between waterinsolubility and narcotic action; but since solubility in water and solubility in organic solvents-e.g., in the esters of higher fatty acids which form the organic solvents of protoplasm-have similarly reciprocal relations. this consideration does not enable us to decide whether a solution-effect or an adsorption-effect is the essential factor in the physiological action. The recent investigations of Langmuir and Harkins on adsorption ${ }^{2}$ indicate, how-

${ }^{1}$ Cf. Traube, "Theorie des Haftdrucks und Lipoidtheoric," Biochem. Zeitschrift, LIV (I9I3), 305.

${ }^{2}$ Langmuir, Journal of the American Chemical Socicty, XXXix (I9I7), I848; XL (I9r8), I36r; Harkins, Clark, and Roberts, ibid., XLII (I920), 700; Harkins and Cheng, ibid., XLIII (I92 r), 35. 
ever, that there is no fundamental difference between these two processes; the affinity for water seems dependent usually on the terminal or polar group of the organic compound ( $\mathrm{COOH}, \mathrm{NH}_{2}, \mathrm{OH}$, etc.), and an adsorbed compound may be one in which part of the molecule has an affinity for (equivalent to solubility in) water, while the other part has not, but is attracted more strongly by the other phase. In such cases the position at an interface may be the chief position of equilibrium, and the predominant effect may be adsorption, with limited solution in either phase. Such a view implies that the transition from adsorption to partition is a continuous one, and explains why highly surface-active compounds usually have high lipoid-water partition-coefficients. Such compounds will enter into solution in the non-aqueous phase, provided this is also a solvent. They may, however, condense at the surface of material in which they do not dissolve, and in so doing influence chemical action at such surfaces. Traube calls attention to the fact that the catalytic action of finely divided non-solvent materials like carbon and platinum may be thus influenced; and he places narcotics in the class of "anti-catalysers"; i.e., they are regarded as decreasing the catalytic and hence the chemical activity of living matter by some form of surface-action, e.g., by occupying the interfacial positions (where chemical activity appears to be greatest) in the heterogeneous protoplasmic system and displacing the chemically reactive compounds. ${ }^{2}$ This view, while partial, may well be correct in certain cases, although it s "Über Katalyse," Arch. ges. Physiol., CLIII (19I3), 309.

${ }^{2}$ Compare Warburg, Biochem. Zeitschrift, CXIX (I92I), I34; see footnote, p. 206. 
probably does not cover the entire range of phenomena included under narcosis.

\section{INFLUENCE OF ORGANIC NARCOTICS ON THE CHEMICAL REACTIONS IN PROTOPLASM}

The fact that narcotic compounds arrest spontaneous activity, and in general act as depressants of vital processes and of irritability, shows that they interfere with the energy-yielding chemical reactions of protoplasm. The essential problem relates to the means by which this effect is produced, whether it is primary or secondary; i.e., there are the alternative possibilities: (I) that the primary action may be a modification of the structural conditions on which the chemical reactions depend; and (2) that the reactions themselves may be influenced directly; e.g., by some form of anticataly tic action.

Warburg and his associates have made an extensive study of the influence of narcotizing compounds on the oxygen consumption of living cells, and the results of this work show many striking parallels with those already described. The cells used in the various determinations included sea-urchin eggs, erythrocytes (chiefly of birds), yeast, lymphocytes (from thymus), spermatozoa (of fishes), liver cells (of frog and mouse), and bacteria (Vibrio, Staphylococcus, Bacillus Typhi). ${ }^{\mathrm{I}}$ In all cases the rate of oxygen consumption was decreased, reversibly, in the presence of a sufficient concentration of anæsthetic. The facts point in general to some kind of physical rather than specifically chemical interference with the oxidation reactions. Thus the effect produced by a particular compound is largely independent of the chemically

${ }^{x}$ For a summary of this earlier work of Warburg, see $\mathbf{M}$ ï incls. med. Wochenschr., LVIII (I9II), 289; also Warburg and Wiesel, loc. cit. 
characterizing group; e.g., two nitriles may be of very unequal effectiveness-require different concentrations to produce the same degree of depression, although possessing the same polar group-and the same is true of other compounds. In any series the depressant action on oxidation is greater with the higher members of the group; and the relative effectiveness of the different compounds is closely similar to that observed in experiments on narcosis. For example, the several alcohols were found to lower the oxygen consumption of birds' erythrocytes by about 50 per cent in solutions of the following concentrations;' Overton's determinations of the minimal anæsthetizing concentrations of the same compounds for tadpoles ${ }^{2}$ are cited for comparison:

\section{Concentration of Solutions Concentrations (Molecular) DEPRESSING $\mathrm{O}_{2}$ CONSUMP- REQUIRED FOR AN ESTHESIA}

ALсоноL TION BY $50 \%$ OF TADPOLES

$$
\text { By weight Molecular }
$$

$\begin{array}{lcll}\text { Methyl..... } & \text { I6 } & 5 \mathrm{~m} & 0.52-0.62 \mathrm{~m} \\ \text { Ethyl...... } & 7.3 & \text { I.6 m } & 0.27-0.3 \mathrm{I} \mathrm{m} \\ \text { Propyl..... } & 5 & 0.8 \mathrm{~m} & 0.1 \mathrm{I} \mathrm{m} \\ \text { N-butyl.... } & \text { I. I } & 0.15 \mathrm{~m} & 0.038 \mathrm{~m} \\ \text { I-butyl..... } & \text { I. I } & 0.15 \mathrm{~m} & 0.045 \mathrm{~m} \\ \text { Amyl...... } & 0.4 & 0.045 \mathrm{~m} & 0.023 \mathrm{~m}\end{array}$

As an example of experiments with bacteria (Vibrio Metschnikovii) the following series may be cited; to diminish oxygen consumption by about half the following concentrations of urethanes were required:

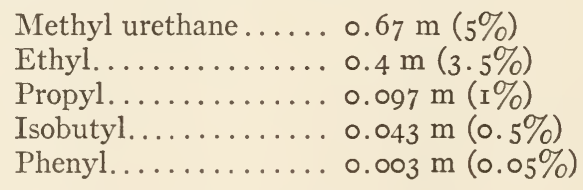

IZ. physiol. Chem., LXIX (rgro), 452.

'Studien über die Narkose, p. ror. 
The relative effects of these compounds on the anaërobic growth of yeast were similar; the following solutions produced about the same degree of inhibition:

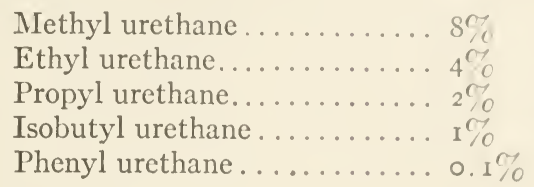

These results ${ }^{\mathrm{I}}$ are similar to those of Regnard with alcohols, cited above.

Usui, working under Warburg's direction, ${ }^{2}$ found also a decrease in the oxygen consumption of vertebrate tissues (liver, central nervous system) under the influence of narcotic compounds (alcohols, ketones, urethanes, methyl urea, and phenyl urea); but in order to produce marked depression of oxidations much higher concentrations were required than in normal reversible narcosis, and the effect was imperfectly reversible. This result is interesting as indicating that anæsthesia is not necessarily associated with a decrease of intracellular oxidations, as Verworn and others have supposed; in fact, Warburg, Winterstein, Loeb and Wasteneys, and others have shown in a number of instances that anæsthesia when perfectly reversible does not necessarily involve a decrease in oxygen consumption. ${ }^{3}$ Diminished oxidation is to be regarded rather as a secondary consequence than as a cause of narcosis. Apparently the chemical

${ }^{2}$ Warburg and Wiesel, loc. cit.

${ }^{2}$ Usui, Arch. ges. Physiol., CXLVII (r912), roo.

3 Warburg, Z. physiol. Chem., LXVI (1910), 305; LAX (1911), 4I3; Winterstein, Biochem. Z., LXI (19r4), 8r; also Winterstein's book on narcosis; Loeb and Wasteneys, Journal of Biological Chemistry, XIV (I9I3), 517; Biochem. Zeitschrift, LVI (I9I3), 295. 
effect is secondary to some physical modification produced in the protoplasm by the narcotizing compound. ${ }^{\mathrm{I}}$

\section{PHYSICAL CHANGES PRODUCED BY LIPOID-SOLVENT COMPOUNDS IN PROTOPLASM}

Certain definite changes in the physical properties of protoplasm, analogous in many respects with those produced by salts, have been observed in various cases to accompany the action of narcotizing compounds; these changes indicate that underlying narcosis there are definite modifications of the structural conditions in protoplasm; and presumably it is to such modifications that the changes in physiological properties and activity are to be referred. As we have seen, the distinctively vital processes are controlled by structural conditions; structural change implies physiological change.

${ }^{s}$ For a more detailed discussion of the relation of narcosis to oxidation processes see my review, "The Theory of Anæsthesia" (Biological Bulletin, XXX (rgr6), 3I I, also American Yearbook of Anesthesia, I, I).

According to Warburg (cf. his recent article on the physical chemistry of cell-respiration, Biochem. Zeitschrift, CXIX [192r], I34) the protoplasmic oxidations occur at the surface of the solid cell structures, which adsorb the water-soluble oxidizable compounds; narcotics influence oxidations by changing the physical and chemical character of the surfaces.

He expresses his general conclusions on the conditions of protoplasmic oxidations as follows: "Two chief means are employed by the cell to diminish the chemical resistance at the regions of oxidation; namely, adsorption and the catalytic action of heavy metals .... Cell respiration is a capillary process occurring at the iron-containing surfaces of the solid cell-constituents. By adsorption at these surfaces the inert organic compounds become capable of reacting with $\mathrm{O}_{2}$ just as do aminoacids at the surface of charcoal. This view does not explain respiration in the physical sense, but classes it with general phenomena of the inorganic world. .... Narcotics check the cell-oxidations by occupying the surfaces and thereby displacing the oxidizable compounds. The same action is exhibited by different narcotics when the same fraction of the active surface is occupied by the narcotic" (pp. I52, I53). 
Changes of permeability, of viscosity, and of resistance to the action of cytolytic or other injurious conditions are the most evident physical effects produced by lipoid-alterant compounds in living protoplasm. 1) uring narcosis there appears very generally to be a decrease of permeability, an increase in the resistance to structural breakdown or cytolysis, and an increase of protoplasmic viscosity. From the general nature of these changes it would seem that the structural substratum of the living matter assumes temporarily a denser or physically more stable condition. In any event it is clear that the modification is in such a direction as to interfere with stimulation, a process, which (as we shall see later) involves structural changes in the irritable system. Hence what may be described as a stabilization, decrease of susceptibility to structural change, is indicated as in all probability the essential physical condition underlying narcosis; but any such general term gives little indication of the detailed nature of the physical modification produced in the living protoplasm. The manner in which narcosis modifies stimulation-processes will be considered in more detail later; in the present section we shall merely describe briefly those changes in the physical state of protoplasm which appear to have some bearing on the question of the nature of the conditions determining narcosis.

The changes observed in the larva of Arenicola are simple and instructive; normal larva transferred from sea water to pure isotonic $\mathrm{NaCl}$ solution undergo stimulation and marked increase of permeability as shown by loss of pigment; on the other hand, larva which are first placed in a solution of a magnesium salt, or in sea 
water containing a suitable anæsthetic (ether, chloretone, alcohol) in the narcotizing concentration, and are then transferred to $\mathrm{NaCl}$ solution (preferably containing anæsthetic), show no such effect; there is no immediate loss of pigment and little or no stimulation. The breakdown of cilia in the $\mathrm{NaCl}$ solution is also prevented. ${ }^{x}$ A protective (antitoxic) or stabilizing action is associated with the narcotizing action in this organism; and essentially similar conditions have been found in the eggs of the sea-urchin Arbacia. ${ }^{2}$ Analogous observations have been made on other cells by a number of investigators. Arrhenius and Bubanovic found that the breakdown of blood corpuscles in hypotonic media was hindered by anæsthetics; ${ }^{3}$ similar observations have more recently been made by Linzenmeier and Runnström; hæmolysis and agglutination by foreign proteins may also be diminished by anæsthetics. ${ }^{4}$ These "stabilization" effects are observed in the concentrations corresponding to the anæsthetizing range; higher concentra-

${ }^{3}$ R. S. Lillie, American Journal of Physiology, XXIX (I9I2), 372; XXXI (I9I3), 255.

${ }^{2}$ R. S. Lillie, American Journal of Physiology, XXX (IgI 2), I.

3 Publications of Nobel Institute (I9I3), No. 32, cited from Höber's Physikalische Chemie der Zelle u. der Gewebe, p. 466.

${ }^{4}$ See Linzenmeier, Arch. ges. Physiol., CLXXXI (I920), I69, and CLXXXVI (I92I), 272; for similar observations on bacteria cf. Vorschütz, ibid., CLXXXVI (I921), 29o; Runnström, Biochem. Zeitschrift, CXXIII (I92I), I. See also the observations of Traube (Biochem. Zeitschrift, X [1908], 37I) and Clowes (Proceedings of the Society of Experimental Biology and Medicine, XI [1913], 8). These changes of properties resulting from the modification of surface-films have an interesting relation to those produced by addition of proteins to suspensions of bacteria and blood corpuscles, and recently investigated by Northrop and de Kruif (Jour. Gen. Physiol., IV [1922], 655), Eggerth and Bellows (ibid., p. 669), and Coulter (ibid., p. 403). 
tions produce irreversible structural change or cytolysis. Such facts indicate, in general, that in the narcotizing solutions the plasma membranes become more resistant to alteration. Höber's observations on the action of anæsthetics in hindering the production of injurycurrents in muscle by potassium salts illustrate the same condition; the local negativity (an index of increase of permeability) develops much more slowly in the presence of ether, urethane, and other narcotizing compounds than in the pure solution. ${ }^{\mathrm{T}}$

A decrease of permeability to water-soluble diffusing substances and ions, and also in some cases to water, is an effect of a related kind. The entrance of watersoluble dyes into plant cells (Spirogyra) is retarded during anæsthesia;2 neutral salts may also produce this effect both in animal and plant cells. ${ }^{3}$ Decreased permeability to ions is indicated by decreased electrical conductivity; this has been demonstrated by Osterhout in Laminaria, by McClendon in sea-urchin eggs, and by Joel in blood corpuscles. ${ }^{4}$ In Laminaria a reversible decrease of conductivity is found only in moderate concentrations of ether and other anæsthetics, corresponding to the anæsthetizing concentrations; stronger solutions cause marked and irreversible increase of

I Höber, Arch. ges. Physiol., CVI (1905), 599.

${ }^{2}$ Lepeschkin, Ber. deulsch. botan. Ges., XXIX (I9II), 349.

${ }^{3}$ Szucs, Jahrb. wiss. Botanik, LII (IgI 2), 85. Cf. also the recent observations by Miss Irwin, Jour. Gen. Physiol., V (1923), 223, 727 . $\mathrm{Mg}$ salts decrease the rate of penetration of dyes into Arenicola larvac (American Journal of Physiology, XXIV [rgog], 26).

4 Osterhout, Science, XXXVII (19I3), III; The Plant Woorld, XVI (1913), I29; McClendon, Popular Scientific Monthly (1915), p. 509; and Joel, Arch. ges. Physiol., CLXI (I9I5), 5. 
conductivity, indicating a destructive or cytolytic effect. In fertilized sea-urchin eggs, anæsthetics (chloral, hydrate, alcohols, urethane) decrease the permeability to water, as shown by the decreased rate of shrinkage in hypertonic sea water containing the anæsthetizing compound. ${ }^{\mathbf{x}}$ The penetration of acids into the pigmentcontaining mantle cells of nudibranchs is also retarded by anæsthetics. ${ }^{2}$

Changes of protoplasmic viscosity, as indicated by changes in the readiness with which cell structures are mechanically displaced (by centrifuging), have also been observed, but the character of the change appears to vary in different forms of protoplasm. In plant cells, according to the observations of Heilbronn ${ }^{3}$ on seedlings and F. Weber ${ }^{4}$ on Spirogyra, ether in the anæsthetizing concentrations increases the viscosity of the protoplasm; in lower concentrations, on the other hand, it decreases viscosity. This result agrees with the observations of Ewart ${ }^{5}$ and others who find that weak solutions of anæsthetics accelerate protoplasmic streaming while stronger solutions retard or arrest it. In sea-urchin eggs L. Heilbrunn ${ }^{6}$ has recently found that various anæsthetics in concentrations sufficient to prevent. cell-

${ }^{x}$ R. S. Lillie, American Journal of Physiology, XLV (IgI8), 406; cf. p. 427.

${ }^{2}$ Crozier, Jour. Gen. Physiol., IV (1922), 723.

3 Heilbronn, Jahrb. wiss. Botanik, XLIV (1914), 357.

${ }_{4}^{4}$ F. Weber, Biochem. Zeitschrift, CXXVI (192I), 21; Ber. deutsch. botan. Ges., XL (1922), 2 I2.

${ }_{5}^{5}$ Ewart, On the Physiology and Physics of Protoplasmic Streaming in Plants, Oxford (1903). Cf. also the observations by Demoor and others cited in Czapek's Biochemie der Pflanzen, Jena (1913), p. г6r.

${ }^{6} \mathrm{~L}$. Heilbrunn, Biological Bulletin, XXXIX (I920), 307. 
division cause decrease of viscosity, i.e., facilitate the displacement of granules by the centrifuge. In some cases, however, Heilbrunn found effects of the opposite kind; and he distinguishes two kinds of anasthesia, in which protoplasmic viscosity is respectively increased and decreased. The significance of such changes in relation to the functional activity of the cell is not clear. They show, however, that the structural conditions within the protoplasmic system are modified reversibly by the lipoid-solvent group of compounds and that the concentrations required for this effect correspond to those which produce narcosis.

Apparently the most general inference to be drawn from the foregoing facts is that one constant accompaniment of narcosis is a modification, in the direction of greater stability or impermeability, of the physical state of the plasma membrane of the irritable cells; and there is good reason to believe that the physiological effect of narcotic compounds depends on this effect; this conclusion will receive further support when the subject of stimulation is discussed. The chief locus of action of anæsthetics thus appears to be the same as that of salts, which is evidently superficial, as already pointed out. Antagonisms between salt action and anæsthetic action are in fact readily demonstrable in many cases. ${ }^{1}$ Salts like $\mathrm{NaCl}$ in pure solution tend to disintegrate the plasma membranes, and the addition of an anasthetizing compound to the solution frequently retards or prevents

I See my series of papers on antagonisms between salts and aniesthetics, American Journal of Physiology, XXLX (1912), 372; XXX, I, and XXXI (19r3), 255; also Journal of Experimental Zoölogy, XVI (1914), 59r. Höber's observations (above cited) on the effects of aniesthetics in retarding the production of injury-currents in muscle by salts furnish other examples of this phenomenon. 
this action in a manner resembling that of an antagonistic salt like $\mathrm{CaCl}_{2}$. Clowes has shown that in the physical drop-systems which he studied-alkaline $\mathrm{NaCl}$ solution flowing from a stalagmometer through oil-anæsthetics produce an effect closely comparable with that of $\mathrm{CaCl}_{2}$; both actions are to be referred to changes in the properties of the interfacial films formed between the oil and the aqueous solution. ${ }^{\mathrm{I}}$ The presence of a compound ( $\mathrm{Ca}$ soap, or fat-solvent compound) which is more soluble in the oil phase than in the water phase, modifies the conditions at the boundary in the same manner in both cases, and produces the same physical effect in the system. The parallelisms observed by Clowes between the biological and the physical phenomena may be interpreted as indicating that the conditions in living protoplasm are of a closely analogous kind.

The foregoing effects of anæsthetics on the physical properties of protoplasm do not, however, enable us to decide whether the solvent action or the adsorbent action is the chief factor in the narcotic effect, or whether both are equally concerned. Certain widely general biological phenomena do not appear to be entirely consistent with Traube's theory that surface-activity is the essential factor in all cases of narcosis. These are: (I) the great differences observed between the narcotizing concentrations of the same compound in different cells, tissues, and organisms; (2) the fact that weak solutions of many narcotic compounds have a sensitizing or accelerating influence on many cell-processes;2 (3) the

'Clowes, Journal of Physical Chemistry, XX (I916), 407; cf. pp. $434 \mathrm{ff}$.

${ }^{2}$ Cf. the instances cited in my review of the theory of anæsthesia (Biological Bulletin, loc. cit.). 
general relation, pointed out by Overton and Meyer, between the lipoid-content of tissues and their susceptibility to narcotic action; and (4) the anxsthetizing action of compounds, such as $\mathrm{Mg}$ and $\mathrm{K}$ salts, which are without surface-activity in the foregoing sense. Traube's theory in fact, in emphasizing the importance of a single physical factor, seems to disregard other possible factors, and on the whole to underestimate the complexity of the physiological conditions.

Höber, ${ }^{x}$ Vernon, ${ }^{2}$ and others have pointed out various exceptions to the rule that isocapillary solutions have equal physiological action. This rule cannot be true in any precise sense, since an organic compound may affect the conditions at the various interfaces in a complex system like protoplasm, and at an air-water interface, quite differently; and other factors, including viscosity, solubility in the protoplasmic phases, and specific chemical affinities enter to modify the simple surface conditions. In cases where the conditions are simple, the increase from compound to compound in a homologous series may be very regular; a good example is seen in Fühner's observations on hæmolysis by solutions of alcohols; ${ }^{3}$ the critical hæmolytic concentrations for the first five alcohols are as follows:

\begin{tabular}{|c|c|}
\hline & m. sol. \\
\hline Methyl. & $7 \cdot 34$ \\
\hline Ethyl. . & 3.24 \\
\hline Propyl. & I.08 \\
\hline Butyl. . & 0.312 \\
\hline Amyl. & 0.09 \\
\hline
\end{tabular}

${ }^{x}$ Höber, Physik. Chom. d. Zelle, p. 415.

${ }^{2}$ Vernon, Biochem. Zeitschrift, LI (1913), I.

3 Fühner and Neubauer, Arch. exper. Path. w. Pharmakcl., I.VI 1907), 333 . 
In this case, the ratio of about $3:$ I between successive members is well shown. In other cases this relation is obscured by chemical and other factors; an instructive instance is the relatively high toxicity of methyl as compared with ethyl alcohol, apparently a consequence of the special properties of the former's oxidation products, formaldehyde and formic acid. Fühner cites other cases showing a similar regularity; he finds, however, that in tissues with high lipoid-content, such as the vertebrate central nervous system, the higher members of certain series (alcohols) are more effective than would be expected from this simple rule. This discrepancy he ascribes to the larger proportion of organic solvent (lipoid) in such tissues; thus, in the adult frog the divergence from the $3: I$ ratio is greater $(=c a .4: I)$ than in the tadpole $(=2.9: I)$; this difference is apparently referable to the increase in lipoid constituents as the central nervous system develops. ${ }^{\mathrm{I}}$ It seems probable that in lipoid-rich tissues the lipoid-solvent factor becomes relatively important in comparison with the capillary constant factor.

The relatively great solubility of many anæsthetic organic compounds in protein-containing systems deficient in lipoid (serum, finely divided muscle, etc.) has been attributed by Moore and Roaf ${ }^{2}$ to the formation of chemical combinations with the protein; but since all such systems are undoubtedly polyphasic, and since chemical combinations (in the stoichiometric sense) of hydrocarbons (like $\mathrm{CHCl}_{3}$, benzol, etc.) with proteins are

× Fühner, Z. Biol., LVII (191 2), 465.

${ }^{2}$ Moore and Roaf, Proceedings of the Royal Society, B, LXXIV (I908), 382; LXXVII (1906), 86. 
difficult to conceive, it seems more likely that an adsorption-effect is involved, similar to that observed in finely divided suspensions of charcoal. It is well known that the catalytic effect of such suspensions is markedly influenced by surface-active compounds; ${ }^{\mathrm{I}}$ this effect indicates an alteration in the character of the surface, probably resulting from adsorption. Other phenomena of a related kind, e.g., the precipitation produced by organic solvents in protein solutions (as observed by Battelli and Stern and Moore and Roaf), ${ }^{2}$ the liquefying action of these compounds on gelatine gels (Traube and Köhler), ${ }^{3}$ the solidifying action on lecithin suspensions, and the interference with the precipitation of lecithin suspensions by electrolytes (Koch, Höber and Gordon, and others), ${ }^{4}$ are similarly referable to surface-conditions, although the special nature of these conditions is not clear in all cases. It is noteworthy that most of these effects are observed at concentrations far in excess of those required to produce reversible narcotic effects in living protoplasm. To characterize the organic anæsthetics as negative catalyzers, as Traube does, may place them in a class, but does not explain their characteristic action on living matter.

It seems certain from the physical peculiarities of these substances that they must undergo adsorption

${ }^{2}$ Cf. Warburg, Arch. ges. Physiol., CLV (19r4), 547.

${ }^{2}$ Battelli and Stern, Biochem. Zeitschrifl, LII (1913), 226; Moore and Roaf, loc. cit.

3 Traube and Köhler, Intcrnat. Zcilschr. f. physik.-chcm. Biol., II (I9I5), 42.

${ }_{4}$ Cf. pp. 360 ff. of my Theory of Anesthesia, loc. cit. 
at the structural surfaces in protoplasm, and also dissolve, in accordance with their partition-coefficients, in the organic solvents of protoplasm. It is not a case of two incompatible processes; both occur simultaneously, and each contributes to the total effect. Possibly the reversible effects characteristic of low concentrations of anæsthetic substances are the expression of solution in the lipoids, while with higher concentrations the specific structural compounds of the protoplasm, the proteins, are affected through coagulation or other changes due to adsorption, and irreversible effects result. The reversible effects-stimulation or sensitization in very weak, inhibition or anæsthesia in stronger, solutionsare the expressions, respectively, of facilitation and hindrance of the normal metabolic processes underlying stimulation and automatic activity; i.e., the influence of the cell-structure on the metabolic reactions is modified, and the whole behavior of the protoplasmic system is altered correspondingly.

If the influence of structural conditions on cellmetabolism is to be included under the class of heterogeneous or contact catalysis, as many of the foregoing facts indicate, it is evident that a consideration of this type of catalysis and of the manner in which it is influenced by substances of the foregoing kind becomes essential in the further analysis of the conditions in living protoplasm. 


\section{CHAPTER X}

\section{CATALYSIS IN RELATION TO THE CHEMICAL PROCESSES IN LIVING MATTER}

The chemical reactions in protoplasm are under the control of structure, as we have seen, and their velocity is decreased and, in the case of the most characteristically vital reactions, the specific syntheses, is reduced to zero when protoplasmic structure is destroyed. If we class this influence of structure as catalysis, the case becomes one of heterogeneous catalysis, in which the reacting substances are predominantly substances in aqueous solution. Organic solvents, however, are also present, represented chiefly by the lipoids; and, as in all cases of heterogeneous catalysis, the interfacial relations are undoubtedly of primary importance. The permanent structural elements are chiefly protein in composition, probably associated with lipoid; and this fact favors the inference that the interfaces between the solid protein structures of the cell and the adjoining more fluid phases are the site of the biologically essential reactions, and especially of the syntheses. The fact that surfaceactive substances as a class interfere so strongly with these reactions favors this interpretation.

The distinctive syntheses of living matter are those of proteins. These are the syntheses on which specific growth depends, and growth is the fundamental vital activity. It thus appears probable that growth is chiefly a result or expression of synthetic reactions occurring at such interfaces; and the general suscepti- 
bility of protoplasmic processes, including growth, to electrical influences seems to imply that the electrical conditions existing at these interfaces are an essential factor in the control of these reactions.

The general subject of the catalysis of substances in aqueous solution in heterogeneous systems has thus an intimate bearing on the fundamental biological problem which we are considering; and a brief review of the more relevant facts in this field is essential to the further analysis of the conditions in living protoplasm. It must be remembered, however, that the theory of catalysis is still in many respects incomplete, and that many reactions in living protoplasm appear to be determined by other than purely catalytic conditionsusing the word catalysis in the accepted sense of an acceleration in which the catalyzer undergoes no permanent change in the reaction. Induced reactions probably play an important part; and there are apparently also cases where the catalyzer acts by introducing a factor necessary to those special physical conditions-e.g., flow of electric current through the bioelectric circuitwhich control the reaction.

The general parallel between the conditions determining chemical reaction-velocities in general, and those determining the flow of an electric current through a circuit, has often been dwelt upon. ${ }^{x}$ The quantity of material transformed in a reaction, or of current flowing through the circuit is determined: (I) by the intensity of a physical condition, called electrical or chemical

${ }^{x}$ Cf. Moore, Recent Advances in Physiology and Biochemistry, pp. 45 ff.; Mellor, Chemical Statics and Dynamics, p. 25; van't Hoff, Vorlesungen, I, I72, I78; Bredig, Ergebnisse der Physiol., I (1902), I37. 
"potential," whose expression is a furtherance of the change in question; and (2) by the resistance to this change. The general formula $C=P / R$ describes the general conditions, where $C$ signifies either the rate of chemical change (under determined conditions of concentration, temperature, etc.) or the intensity of the current flowing through the circuit, $P$ the potential, signifying a function of "chemical affinity" in the one case, or the electrical pressure or "voltage" of the circuit in the other, and $R$ the resistance to either the chemical change or the flow of current. In the case of a chemical reaction occurring at an electrode (electrolysis), where the quantity of chemical change, e.g., of copper deposited as metal at the cathode, is proportional to the quantity of current flowing through the circuit (Faraday's Law), the factors determining the flow of current are the same as those determining the rate of chemical change, and chemical resistance becomes identical with electrical resistance. In such a case any condition decreasing the electrical resistance or increasing the electrical potential increases the velocity of the purely chemical change at the electrode.

At present it is customary to describe a catalyst as a substance which in some manner, without itself undergoing permanent alteration, decreases the resistance to the interaction of other substances in the reactionsystem. On such a definition any substance which decreases the electrical resistance in a circuit would catalyze the chemical reactions occurring at the electrodes. Such an effect might not ordinarily be classed as catalytic; but since our interest is not in defining the significance to be attached to terms, but in ascertain- 
ing the physico-chemical conditions under which chemical reactions actually are accelerated in systems of the kind under consideration, we must note as especially significant the fact that reactions occurring under electrical influence at surfaces (especially metallic surfaces) may be influenced in their velocity by the contact of materials which change locally the electrical state of the surface. The rusting of iron in water or salt solution is a good instance of this type of effect; the reaction may be greatly accelerated by placing another metal, e.g., copper or platinum (which itself does not undergo change) in contact with the iron. The apparently catalytic effect in this case is due to the formation of an electrical circuit between the two metals, the iron becoming anodal and hence freeing Fe ions with increased rapidity; these can then react to form carbonate or hydrate with the anions present in the solution. Another simple and striking demonstration of a "catalytic" action of this kind is made by placing in a solution of $\mathrm{K}_{3} \mathrm{FeCy}_{6}$ (containing a little $\mathrm{NaCl}$ to allow a soluble zinc salt to be formed) two similar strips of metallic zinc, one of which is marked with a lead pencil or bound with a small piece of copper or platinum, while the other is free from such contact. In a few hours a luxuriant "growth" of filaments and tubules of zinc ferricyanide is formed from the first strip, while the second remains almost unaltered. The carbon, or the noble metal, acts "catalytically" in this reaction because it furnishes a surface of lower solution-tension, which forms the cathode of the local electric couple; and since these two areas are in metallic connection and immersed in the electrolyte solution, a current flows which enables 
$\mathrm{Zn}$ ions to enter the solution more rapidly and hence accelerates the formation of the structure-forming precipitate of zinc ferricyanide. ${ }^{\mathrm{r}}$

There is reason for believing that the remarkible chemical activity of living matter, as well as its susceptibility to electrical influence and to stimulation, is largely dependent on physical conditions which are fundamentally of the kind just described. For example, during stimulation the excited and the unexcited areas of the reactive protoplasmic surface-the surface of the irritable cell, neurofibril, or other structure concernedare at different electrical potentials; apparently the current flowing between these two areas produces chemical effects which secondarily determine the propagation of the state of excitation and hence the distinctively physiological effect or response. There is a close analogy here to the case of local circuits in metals immersed in electrolyte solutions; these circuits also form the condition for the transmission of chemical effects. This general condition will be considered more fully under the subject of stimulation; at present it is sufficient to call special attention to it as probably forming a highly important factor in the catalytic or quasi-catalytic action of living protoplasm. Here we use the term "catalytic" simply as a designation for the remarkable property shown by living protoplasm of enabling reactions to occur, at a relatively high velocity, which are absent or inappreciable in dead protoplasm.

The most familiar form of catalysis observed in living organisms, and the one showing the closest parallels

${ }^{2}$ Cf. my two papers on precipitation growths from metals, Biological Bulletin, XXXIII (1917), I35, and (with E. N. Johnston) XXXII (1910), 225. 
with the usual inorganic types of catalysis, is that dependent on the activity of enzymes. Enzymes are constant constituents of protoplasm, and their presence accounts for many characteristic features of its chemical performance. Enzyme-action, however, is obviously responsible for only a portion of the metabolic reactions, especially the destructive or disintegrative ones, which are largely hydrolytic. Although certain types of synthesis are accelerated by enzymes under certain conditions (dehydrolytic synthesis of esters, carbohydrates, and apparently polypeptides), others cannot be thus accounted for; e.g., photosyntheses, synthesis of fat from protein or carbohydrate, or other syntheses involving the expenditure of much energy. Moreover, the responsiveness of protoplasm to stimulation is not thus explained, since enzymes show no such instantaneous and marked acceleration of their action, under electrical or mechanical influence, as is shown by living protoplasm. As we shall see later, changes in protoplasmic structure seem to be primarily responsible for the immediate chemical effects following stimulation.

Enzymes are simply colloidal catalyzers of complex and specific chemical constitution. A part of their catalytic activity presumably depends on their colloidal state; i.e., a state of subdivision making surface-conditions of preponderant importance in their chemical behavior. The general conditions of heterogeneous catalysis thus apply to enzyme action; in addition there are special conditions referable to the specific stereochemical configuration of the enzyme molecule.

It is well known that finely divided material of various kinds (material with large surface-extent) is often very 
active in catalyzing chemical reactions; this is especially true of certain forms of carbon and of metals like platinum. The action of platinum is especially well known; it increases with the state of subdivision, i.e., the extent of surface, hence colloidal platinum is a very effective catalyzer. Other metals have similar properties, although usually less marked.

Usually in such heterogeneous catalyses the acceleration of reaction-velocity is regarded as a result of increased concentration at the surfaces. Faraday (1839) suggested this explanation for the action of platinum in catalyzing the combination of hydrogen and oxygen. In general, when considering any special case of heterogeneous catalysis, three independent processes with different rates are taken into account: (I) the rate of diffusion of the dissolved substrate to the active surface; (2) the rate of adsorption at the surface; and (3) the rate of chemical combination. The rate of reaction is limited by the rate of the slowest of these interdependent processes. In most cases the reaction-velocity $(V)$ is regarded as determined by the concentration $(C)$ attained at the surface and by the specific velocityconstant $(K)$ of the reaction (i.e., $V=K C$ ), since adsorption is rapid and also diffusion (when the distances are small). The rate of chemical change is increased (catalytic effect) because the concentration of the reacting molecules is increased in this part of the system. ${ }^{x}$

It is evident, however, that other factors frequently if not usually enter dependent on the special chemical nature of the reacting compounds. Many inorganic

${ }^{\mathrm{C}} \mathrm{Cf}$. Höber's Physik. Chemie der Zelle, pp. 702 ff., for an account of the general conditions of catalysis in heterogeneous systems. 
catalyses are referable to the formation of intermediate compounds, and the same is undoubtedly true of many enzyme-reactions. The specificity of enzymes and other facts in their behavior indicate that chemical union often occurs between the enzyme and the substrate molecules, and that it is the combination thus formed which breaks down rapidly, yielding the products of hydrolysis and the free enzyme, which then repeats the chemical cycle of combination and hydrolysis with fresh molecules of substrate.

Apparently many cases of heterogeneous or contact catalysis are referable to simple increase of concentration due to adsorption. But in the case of metals and other conducting substances the possibility of an additional factor, the formation of local electrical circuits between different portions of the active surface, is also to be considered. In either case the essential condition is some form of surface-action.

Enzymes are colloidal in their condition, indiffusible, precipitable, readily adsorbed by indifferent adsorbents, and, according to Bayliss, their mode of action is also a surface-action. The clearest proof of this is that emulsin, lipase, urease, and trypsin exert their action in alcoholic media of such a strength that the enzyme is insoluble and can be filtered off. ${ }^{\mathrm{I}}$ Bayliss regards the adsorption of the substrate on the enzyme phase as the first step in the process; the chemical reaction then follows. In some cases the adsorption-compound of enzyme with substrate is separable; e.g., starch-amylase, fibrinpepsin, and trypsin with caseinogen. ${ }^{2}$ A close union or

${ }^{r}$ Cf. Bayliss, Principles of General Physiology, p. 325.

2 Bayliss, op. cit., p. 326. 
adhesion of the enzyme to the substrate is characteristic; and the influence of electrolytes on enzyme-processes is probably in large part to be referred to their influence on adsorption. ${ }^{\mathrm{x}}$

In the simple adsorption type of catalysis the accelerating effect depends on the concentration attained at the interface. Close adhesion of the reacting compound to the adsorbent surface is important since this implies a high concentration at the surface. Hence a correspondence between the molecular configuration of the adsorbing surface and of the adsorbed compound is favorable to adsorption as well as to chemical combination. The importance of such conditions is seen in the growth of crystals, in which, according to Marc, the dissolved molecules are abstracted from the motherliquid and deposited on the surface of the crystal by a process identical with adsorption. ${ }^{2}$ Slow growth is favorable to the formation of large crystals, because time is then allowed for the regular orientation of the surface-molecules thus deposited. Organic growth apparently also depends on the apposition of newly formed molecules to the similarly constituted molecules already laid down in the solid state as structure; and this consideration may explain why, in living organisms, where definiteness of form and of structural characters is essential, the rate of growth is slow. Probably no essential distinction is to be drawn between adsorption and chemical combination; in adsorption the surface molecules of the adsorbent are alone concerned because

${ }^{x}$ Cf. Bayliss, "Adsorption as a Preliminary to Chemical Reaction," Proceedings of the Royal Sociely, B, LXXXIV (IgIr), 8I.

${ }^{2}$ Marc, op. cit. 
of the solidity of the adsorbing phase and the remoteness of the internal molecules from the sphere of reaction.

\section{SPECIAL FACTORS IN THE CATALYTIC ACTION OF PROTOPLASM}

Since surface-relations are all-important in heterogeneous catalysis, all conditions modifying the composition, electrical polarization, or other characters of the active surfaces influence the catalytic activity of the system. Hence surface-active substances as a class have a marked effect on such catalyses, and their influence on the chemical activity of living matter is undoubtedly in large part referable to this effect. A brief review of the action of these substances on the catalytic and other properties of heterogeneous systems will indicate the nature of the factors.

One of the most complete recent studies of the anticatalytic action of surface-active substances on enzyme action is that of Warburg and Wiesel ${ }^{\mathrm{I}}$ on the zymasecontaining "press-juice" of yeast. All substances of the anæsthetic class retard the alcoholic fermentation caused by this enzyme, although the non-living enzyme requires higher concentrations than the living cell for the same proportional degree of retardation. In homologous series the concentration necessary for a given retardation decreases in the usual manner with increase of molecular weight. The following orders of relative action were found for different compounds. Alcohols: methyl<ethyl<propyl<isobutyl; urethanes: ethyl< propyl <isobutyl; nitriles: aceto $<$ propio $<$ isovalero; ketones: acetone $<$ methyl-propyl $<$ methyl-phenyl. It

$\therefore$ Warburg and Wiesel, loc. cit. 
was also noted that all of these compounds in sufficient concentration caused precipitation in the press-juice, and that the orders of relative precipitating effectiveness and anticatalytic action were the same; this order is also that of relative narcotic action. This parallelism between precipitating action and narcotic action recalls Claud Bernard's hypothesis that a partial coagulation of protoplasmic constituents is the essential condition of narcosis. $\quad$ With the living cell, however, much lower concentrations are required to stop fermentation than with the enzyme solution, so that the parallel between the inactivation of the structureless enzyme solution and the inhibition of fermentation in the living cell is not complete. This difference may indicate the importance of the vital organization as such, or it may depend on the presence of special compounds (lipoids) in the living cell. Warburg and Wiesel found, however, that dried yeast cells (extracted with ether and acetone) exhibited a well-marked fermentative action, which was arrested by narcotic compounds in somewhat high concentrations. Meyerhof found a closely similar anticatalytic action of the same compounds in solutions of yeast invertase; and in this case also the effect was associated with a precipitating action; similar observations on oxidasecontaining tissue-extracts have been made by Battelli and Stern. ${ }^{3}$ Vernon ${ }^{4}$ also observed a general inhibitory action of narcotics on tissue-oxidases; the effect was

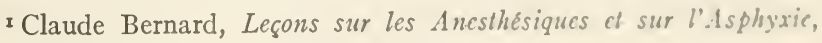
Paris (1875), p. 154 .

${ }^{2}$ Meyerhof, Arch. ges. Physiol., CLVII (Igr4), 25 I.

3 Battelli and Stern, Biochem. Zeitschrift, LII (1913), 226.

4 Vernon, Journal of Physiology, XLV (1912), 197. 
imperfectly reversible and may be regarded as destructive rather than simply anti-catalytic. Narcotics also check heterogeneous catalysis in purely inorganic systems; e.g., the oxidation of oxalic acid by charcoal (Warburg) ${ }^{\mathrm{x}}$ and the decomposition of $\mathrm{H}_{2} \mathrm{O}_{2}$ by colloidal platinum (Meyerhof), ${ }^{2}$ and the same order of relative action is again seen.

The foregoing association of a precipitating action with an anticatalytic action indicates an alteration of surface-conditions, but precipitation as such is not a necessary accompaniment of this action. Many organic compounds (alcohols) precipitate solutions of proteins and other colloidal compounds, but usually the concentrations required for precipitation far exceed the anticatalytic concentrations.

Other effects, also dependent on surface-action, have an intimate bearing on the present problem. Of special interest is the action of narcotic compounds on suspensions of lecithin. Changes in the viscosity, gelforming properties, and precipitability of lecithin emulsions are characteristic. Many lipoid-solvents (alcohols, etc.) increase the viscosity of these emulsions to a greater degree than can be accounted for by the increase in the viscosity of the aqueous phase. ${ }^{3}$ In somewhat concentrated emulsions (IO-I 2 per cent) the addition of ether even causes gelation, so that the test tube can be inverted without spilling; this effect is also caused by alcohols (n-propyl up to capryl), esters (ethyl formate, acetate,

${ }^{x}$ Warburg, Arch. ges. Physiol., CLV (1914), 547.

'Meyerhof, Arch. ges. Physiol., CLVII (19I4), 280.

3 Handowsky and Wagner, Biochem. Zeitschrift, XXXI (I9II), 32; A. Thomas, Journal of Biological Chemistry, XXIII (I9I5), 259. 
propionate, and nitrate), ethyl ether, Et(Cl, EtBr, chloretone, paraldehyde, $\mathrm{CCl}_{4}$, hydrocarbons like benzol, toluol, and xylol, but not by urethanes and lower alcohols. ${ }^{\text {I }}$ In a lecithin-containing system such as protoplasm this influence might act anticatalytically by slowing diffusion-relocities; any chemical process whose rate depended on the rate of diffusion would thus be retarded. Increased hindrance to the movement of ions would be shown in decreased electrical conductivity. Loewe $^{2}$ has in fact found that artificial membranes impregnated with lecithin exhibit an increased electrical resistance in the presence of anæsthetics; in a system like living protoplasm such an effect would retard chemical reactions dependent on electrochemical conditions. The increase in viscosity is probably to be referred in part to the formation of adsorption-films at the surface between the lecithin particles and the water. ${ }^{3}$ It is possible also that changes in the relative volumes occupied by the colloidal particles and the aqueous phase may play some part; presumably the lipoid-soluble compound concentrates in the lecithin in accordance with its partition-ratio, $\frac{\text { lipoid-solubility, }}{\text { water-solubility }}$ and in so doing enlarges the particles; this may explain why the higher alcohols are more effective in causing gelation than the lower alcohols, which are highly water-soluble. As the emulsion particles enlarge, their freedom of movement becomes less, contacts are more frequent. and coherence to a gel is favored.

${ }^{3}$ Cf. my review, The Theory of A nasthesia, op. cil., p. 362 .

${ }^{2}$ Loewe, loc. cit.

${ }_{3}$ Cf. Ibid. 
It should be noted that in the case of some other colloidal organic compounds the formation of gels may be prevented instead of promoted by the addition of surface-active substances; this effect was observed by Schryver ${ }^{\mathrm{I}}$ in the gelation of Na-cholate in the presence of various surface-active organic compounds. He found a retardation in the rate of gelation, the effect running in general parallel with capillary activity and narcotic action. This phenomenon is analogous to protective action in colloidal precipitation.

The protection of suspensions of lecithin against precipitation by electrolytes is also an effect characteristic of many surface-active compounds. Höber and Gordon ${ }^{2}$ found that suspensions containing ether, chloroform, chloral, or amyl alcohol were less readily precipitated by alkali-earth cations $(\mathrm{Ca}, \mathrm{Ba})$ than the control suspensions; i.e., were stabilized; and they characterized this action as "narcotization of the plasma membrane colloid lecithin." Koch and MacLean ${ }^{3}$ found that the stabilizing effect was not uniform with different anæsthetics; some compounds so act, but others are indifferent, while still others further precipitation, especially the lower alcohols and paraldehyde, which are highly water-soluble. My own observations on the precipitation of lecithin by $\mathrm{CaCl}_{2}$ and $\mathrm{HCl}$ confirm this result, but they show that at appropriate concentrations the great majority of anæsthetics have a stabilizing effect. The compounds examined included alcohols 366.

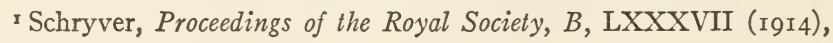

${ }^{2}$ Höber and Gordon, Hofmeisters Beitr., V (1904), 432.

${ }^{3}$ Koch and McLean, Journal of Pharmacology and Experimental Therapeutics, II (I910), 249. 
from n-propyl up, esters, urethanes, hydrocarbons, chloroform, nitromethane, ethyl ether, chloretone, pheny.l urea, and others. ${ }^{\mathrm{x}}$

In general these effects in lecithin suspensions are referable to several factors, of which the chief probably are solution of the compound in the colloidal particles, formation of adsorption-films which change the electrical polarization or other physical constants of the particles, and increase of viscosity. The total effect in most cases is increase in the physical stability exhibited by the system in the presence of conditions tending to alter the state of aggregation.

The protective influence exerted by proteins and other surface-active colloids in the precipitation of metallic hydrosols by electrolytes is apparently a closely related phenomenon. Its conditions have been studied carefully by Zsigmondy, ${ }^{2}$ who finds wide variations in the effectiveness of different compounds; e.g., gelatine is highly effective as compared with peptone. He assigns to each protective colloid a characteristic "gold number": this number defines the quantity of the colloid required to prevent the precipitation of a standard gold suspension by a definite concentration of $\mathrm{NaCl}$. In this case the protective effect undoubtedly depends on the formation of adsorption-films, which prevent coalescence or flocking of particles. Adsorption-films of soap, protein, or similar substances play an analogous part in the formation of emulsions, as already pointed out, the suspended droplets being thus prevented from fusing. In a similar manner these substances prevent sedimentation in finely

${ }^{2}$ Cf. my The Theory of Anasthesia, p. 36r.

${ }^{2}$ Zsigmondy, Colloids and Ultramicroscopy 
divided suspensions of water-insoluble materials (chalk, Ca-phosphate, etc.).

In his textbook ${ }^{I}$ Höber has described various other instances of protective action and has discussed briefly the general biological significance of this phenomenon. In living organisms, where water-insoluble materials constitute an indispensable element in the formation of all permanent structure, protective action is of great importance. Many otherwise insoluble materials are thus kept in a permanent state of fine dispersion or pseudosolution. It is well known that uric acid and cholesterol are present in serum in concentrations far higher than correspond to their solubility in water; presumably they are held in suspension as ultra-microscopic particles by the protective action of the serum proteins. Pauli and Samec ${ }^{2}$ have shown that gelatine, serum albumin, and other proteins may keep large quantities of insoluble calcium salts (sulphate, phosphate, carbonate) in apparent solution. The silver salts in photographic plates and the Ca-phosphate in milk are other instances of insoluble materials kept in fine dispersion by protective colloids. The suggestion has been made that the formation of pathological concretions in higher animals (gallstones, uric acid deposits) is an expression of deficiency in protective colloids. It is probable that in the formation of normal structures, such as bone, by separation of insoluble salts as a finely divided and structurally regular deposit, the protective action of the protoplasmic colloids is a necessary factor; presumably in the absence of this factor the particles would be flocked

r Page 344 .

${ }^{2}$ Biochem. Z., XVII (1909), 235. 
by the salts present and the uniform and gradual building up of regular structure would be impossible.

All of the above-described effects depend on the formation of interfacial films which alter the physical properties of the surfaces. Apparently also the chemical or adsorptive and hence catalytic properties of the surfaces are secondarily altered by the deposition of such foreign materials; and the foregoing evidence indicates that the anticatalytic action of surface-active compounds depends on a surface-concentration of this kind.

Anticatalytic action is, howerer, also well known in homogeneous solutions and even in gases, so that this form of explanation does not always apply. Numerous instances of anticatalysis in homogeneous solutions are cited by Traube in his Theorie der Narkose; Bigelow's observations ${ }^{2}$ on the oxidation of $\mathrm{Na}_{2} \mathrm{SO}_{3}$ by oxygen furnish many striking illustrations; he found that this reaction was depressed by a large number of organic substances; for example, a trace of mannite (about .00I4 per cent) decreased the reaction velocity by 50 per cent; benzyl alcohol and aldehyde, isobutyl alcohol and ethyl alcohol were even more effective in checking the reaction. It is interesting to note that the alcohols showed increasing effectiveness with increasing molecular weight, and that the effect decreased with the number of hydroxyls in the compound (e.g., monohydroxylic alcohols $>$ dihydroxylic $>$ trihydroxylic, etc). Aromatic compounds were more effective than chain compounds. ${ }^{3}$

$\therefore$ Arch. ges. Physiol., CLIII (rgr3), 279.

${ }^{2}$ Bigelow, Z. physik. Chem., XXVI (I89\$), 423 , and XXVII, $5 \$ 5$.

3 Many other similar cases are cited in Traube's paper (the work of Veley, Titoff, Young, and others). 
Anticatalysis in homogeneous solution has the appearance of being a different phenomenon from the forms of anticatalysis considered above, and its conditions are obscure. Possibly the nearest analogies are with photocatalysis; e.g., a catalytic substance may play a rôle analogous to that of a photochemical sensitizer; it is clear that interference with the action of the latter would arrest the photocatalytic action. Just as the chemical effect of light is influenced by the presence of substances with special light-absorptive properties, as in sensitized photographic plates, so also catalysis by chemical compounds may be influenced by other compounds having special chemical relations with the catalyzer. Such considerations are perhaps vague, but since many of the conditions controlling reaction-velocities are imperfectly understood, it seems best not to attempt further definiteness at present. 


\section{CHAPTER XI}

ELECTRICAL AND OTHER FACTORS IN THE:

CATALYTIC ACTION OF PROTOPLASM

Of the conditions, other than temperature and the presence of catalyzers, influencing reaction-velocities, light and other forms of radiation and electricity are the most important. Apparently all chemical reactions are influenced by radiation of appropriate wave-length, and the acceleration caused under these conditions is called "photocatalysis." It differs, however, from the chemical forms of catalysis in that energy is added to the reacting system from without; in this respect the conditions may be compared to those present when an electric current is passed into a solution from an electrode; at the surface of transition between solution and electrode chemical reactions are induced (electrolysis), the effect depending directly on the quantity of electricity (i.e., number of electrons) transferred between the molecules of the electrode and those of the solution. Similarly the chemical effect of light is referred to facilitation of the transfer of electrons between molecules. In virtue of its physical character as electromagnetic oscillation, light alters the range of movement of the electrons; and when the periodicities of electron motion and ethervibration correspond, this range may be increased to a degree sufficient to enable adjacent molecules to interact. The electrons affected are apparently the valence or combination electrons of the molecules concerned. The chemical action of light is thus ultimately to be 
related to its general influence on electrons, an influence shown in the photoelectric effect.

The phenomena of induced reactions, which are almost certainly of great importance in cell-metabolism, are probably also to be affiliated in a general way with photocatalysis and electrolysis (which may perhaps be called "electrocatalysis"); but it is impossible here to do more than direct attention to these possibilities, the investigation of which is the subject of physical chemistry rather than of biology. It is sufficiently evident that all conditions influencing chemical reaction-velocities are of fundamental biological interest.

In considering the case of heterogeneous catalysis (or chemical contact effects) and the influence of anticatalyzers, the effect of the latter on contact-potentials should be noted. These potentials are affected by many organic substances, especially surface-active compounds; ${ }^{\mathrm{I}}$ the strength of the current in a battery and the rate of the associated chemical effects may thus be decreased. For example, in the formation of precipitation-structures from zinc and $\mathrm{Fe}$ under the influence of local circuits, the presence of alcohols, esters, and other surface-active compounds of the anæsthetic groups has a well-marked retarding influence. ${ }^{2}$ The concentrations required for pronounced retardation are similar to those effective in the above-described forms of anticatalytic action, and the effect may be described as anticatalytic, although its conditions are probably complex, the influence on below.

I Cf. the papers of Gouy, Abl, Grumbach, Loeb and Beutner cited

${ }^{2}$ Unpublished observations of my own in Clark University. 
viscosity and on adsorption entering in addition to that on contact-potentials.

In a recent review of the facts and theories of contactcatalysis Bancroft ${ }^{\mathrm{T}}$ cites various instances where electrolysis and electrode-potentials are altered by foreign substances. Thus the P.D. at which $\mathrm{O}_{2}$ is freed at a platinum surface is found to be influenced by the electrolytes present. With platinum electrodes oxidations occur more readily at platinized than at smooth surfaces, apparently because of the "catalytic" action of the finely divided platinum. The presence of cyanide and other compounds reduces the rate of oxidation occurring at an electrode under a given P.D.; for example, a neutral solution of $\mathrm{Na}_{2} \mathrm{~S}_{2} \mathrm{O}_{3}$ is oxidized to tetrathionate at a platinized anode with a P.D. of 0.44 volts and a current-density of $3 \times \mathrm{IO}^{-4}$ amperes per square centimeter. If a trace of $\mathrm{Hg}(\mathrm{CN})_{2}$ is added, the anode P.D. for the same current rises to 0.48 volts. Various salts have marked influence on the electrochemical processes at smooth anodes. ${ }^{2}$ Gouy $^{3}$ made an extensive study of the effects of various compounds on the surface-tension maxima in the capillary electrometer. Usually this maximum corresponds to a minimal P.D. between the $\mathrm{Hg}$ and the $\mathrm{H}_{2} \mathrm{SO}_{4}$; but the P.D. and the surface-tension are both changed by the substance added, so that the position of the maximum is shifted, and this influence was found to be greatest with highly surface-active substances. Similar observations were made by $\mathrm{Abl}$

Bancroft, Journal of Physical Chemistry, XXI (1917), 734.

${ }^{2}$ Cf. Foerster, Eleklrochemie wässriger Lösungen (1915) for further details.

3 Gouy, Ann. de Chim. et de Phys., XXIX (1903), I45; VIII (1900). 29I, and IX, 75 . 
on the electromotive force of cadmium-amalgam cells. ${ }^{x}$ In the case of contact-potentials between water and other dielectrics, Grumbach ${ }^{2}$ found in general that organic compounds which lower the surface-tension of water decrease these potentials. With $\mathrm{CH}_{3} \mathrm{OH}, \mathrm{C}_{2} \mathrm{H}_{5} \mathrm{OH}$, and $\mathrm{C}_{4} \mathrm{H}_{9} \mathrm{OH}$ the effect increases in the order of increasing molecular weight; the curves relating potential change and concentration resemble the corresponding curves of surface-tension and adsorption. The observations of Loeb and Beutner ${ }^{3}$ on the contact-potentials between organic membranes (apple skin) and electrolyte solutions containing alcohols also show a decrease of P.D. with the addition of alcohol. With the first three alcohols the effect increased was in the order of $\mathrm{C}_{\mathrm{I}}<\mathrm{C}_{2}<\mathrm{C}_{3}$ (p. 302). Similar results were obtained with solutions of lecithin in guaiacol. The concentrations required to produce a decided influence on the potentials were, however, much higher than the physiologically effective or narcotizing concentrations.

Traube nevertheless regards the influence of surfaceactive substances on the contact-potentials between the living cell and the medium as an important factor in the physiological effect, and Macallum has expressed a similar view. ${ }^{4}$ It is doubtful, however, if this can be generally true, since the bioelectric potentials are not necessarily decreased in anæsthesia. For example, in pure sugar solution, which produces in muscle effects

${ }^{x}$ Cf. Traube, "Theorie der Narkose," Arch. ges. Physiol., CLIII (1913), 276; cf. p. 303.

${ }^{2}$ Ann. de Chim. et de Phys. (rgrr).

3 Biochem. Zeitschrift, LI (1913), 300.

${ }_{4}$ A. B. Macallum, Surface Tension and Vital Phenomena, University of Toronto Studies (I9I2), No. 8, pp. $68 \mathrm{ff}$. 
similar to anæsthesia, the bioelectric P.D. is increased. ${ }^{x}$ The local negativity produced by anisthetics under certain conditions, as in Allcock's experiments, ${ }^{2}$ is probably to be referred to the permeability-increasing effect of the compounds in higher concentrations

The connection between adsorption and contact catalytic action has been usually regarded as a special case of the mass-action law. Since any increase in the concentration of a reacting substance involves a proportional increase in reaction-velocity, and since the concentration of many dissolved substances is increased in the layer of solution adjoining an interface, it follows that in any such case there must be increased reactionvelocity in that region. Hence if the adsorbing surface is sufficient in area, as with fine subdivision, a large proportion of the reacting material may be present in the surface layer at any time, and a considerable increase in reaction-velocity may result. Direct parallels between adsorption and catalysis have in fact been observed in certain cases. ${ }^{3}$

It is, however, questionable if this influence is in itself sufficient to account for the effects observed; in many cases the surface appears to exercise some specific chemical influence; and certain contact catalyzers, especially platinum, have an accelerative action which is far greater than can be accounted for on the ground of their adsorptive capacity alone. ${ }^{4}$

${ }^{x}$ Cf. Brünings, Arch. ges. Physiol., XCVIII (1903), 24r. Macdonald's work also shows an increase of the demarcation potential with decrease in the external electrolyte content. Cf. p. 304.

2 Allcock, Proceedings of the Royal Socicty, B, LXXVII (1 go6), p. 207.

${ }^{3}$ Cf. Höber's Physik. Chemie d. Zelle (1914), p. 705.

${ }_{4}$ Cf. Bancroft's Applied Colloid Chemisiry, p. 40. 
From the physiological point of view the chief case of specific acceleration by a finely dispersed or colloidal catalyzer is enzyme action. There seems to be no doubt that in many cases the enzyme acts through the formation of intermediate compounds, which break down at high velocity yielding the decomposition products and the original enzyme, the latter then recombining with the substrate molecules to repeat the cycle. The formation of the enzyme-substrate combination presupposes intimate contact between the molecules of enzyme and substrate; hence a correspondence in size and pattern between the intercombining molecules (or parts of molecules; e.g., zymophore groups) is required, of the kind indicated by the "lock and key" comparison of Emil Fischer. That relations of this kind play a part in adsorption is indicated by Marc's observation that a crystalline adsorbent like $\mathrm{BaSO}_{4}$ exhibits preferential adsorption for compounds crystallizing in the same system. ${ }^{\mathrm{I}}$ Apparently this is a simple instance of specific adsorption. Close contact of this kind gives the occasion for chemical transformations (hydrolysis, etc.) which otherwise would not occur, or at much lower velocity. According to Bayliss ${ }^{2}$ a simple reversible adsorption often precedes a more intimate "chemical" union between adsorbent and adsorbed substance. In specific catalyses, like those induced by enzymes, factors of a similar kind probably enter; the union is specific because of the similarity of molecular configuration between enzyme and substrate, and the chemical effect follows because the enzyme-substrate combination is

${ }^{x}$ Cf. Marc, loc. cit.

2 Bayliss, Proceedings of the Royal Society, B, LXXXIV (I9II), p. 269. 
unstable and breaks down in the manner already indicated.

Catalytic effects due to simple increase in concentration are accordingly to be distinguished from those which depend on the appearance of special relations of sume kind between the molecules of the substrate and of the catalyzer. In the latter case the formation of combinations differing from the substrate in reactivity, e.g., velocity of hydrolysis or oxidation, becomes possible; and when these temporary combinations break down, setting free the catalyzer and enabling the latter to repeat the combination, the total effect is the same as if chemical change in the substrate alone were accelerated. The general theory of intermediate compound formation would thus apply to any specific adsorption-catalysis. The adsorbent would correspond to the enzyme, and the theory of such catalysis need not differ in principle from that of catalysis in homogeneous solutions; e.g., $\mathrm{H}$-ion catalysis. At present, however, there appears to be no completely satisfactory theory of this type of catalysis.

With regard to other conditions which may enter in cases of heterogeneous catalysis, it has been suggested that the increased reaction-velocities shown by many substances (sugars, etc.) in protoplasm are phenomena of the same kind as the differences of velocity shown by a given reaction in different solvents, as observed by Menschutkin and others. ${ }^{x}$ Bredig compares a particle of a solid catalytic agent with a drop of liquid, and regards the formation of an adsorption-film as equivalent to

I Menschutkin, Z. physik. Chem., XI (1887), 6II; von Halban, ibid., LXXXII (1913), 325. Cf. Höber, op. cil., p. 704. 
solution; $;$ in this case different reaction-products might be obtained from the same reaction-mixture under the influence of different catalytic agents, differences of adsorption having the same effect as differences of solubility. Selective adsorption and selective catalysis would thus be referred to the same conditions, and both referred ultimately to the same conditions as selective solubility. At present there is an increasing tendency to regard both solution and adsorption as special cases of chemical combination. But although protoplasm contains solvents in which it is conceivable that certain reactions may proceed with increased velocities, it does not seem probable that such considerations can explain the high velocities of the biologically more important reactions, such as the oxidation of sugar. Many facts, especially the phenomena of irritability, show that the conditions determining the increase of reaction-velocities in protoplasm are of a special kind and that the factor of organized structure is all-important. It is not simply a case of transferring a substance from a solvent in which its reaction-velocity is low to one in which it is high.

It has been pointed out by various authors that when adsorption is highly selective, displacements of equilibrium may occur; thus an adsorbent may change the $\mathrm{H}$-ion concentration of a solution. Such effects may be attributed to the selective adsorption of ions (Freundlich); but, as we have seen, the distinction between the adsorption of a substance and its chemical combination with the surface molecules of the adsorbent cannot be

I Cf. Bredig, Ergebnisse der Physiol., I (1902), 2 I I.

${ }^{2}$ Cf. Freundlich, Kapillarchemie. 
sharply drawn. If, in fact, the two processes are indistinguishable, the case becomes essentially one of alteration of equilibrium following the introduction of an additional reagent into a reaction-mixture. Bancroft cites instances where the same compound undergoes different reactions under otherwise similar conditions according to the nature of the contact-agent present; thus with colloidal nickel as catalyzer, alcohol forms acetaldehyde and hydrogen, while with colloidal silica or alumina it forms ethylene and water; and he refers this difference in the catalytic action to the differences in the selective adsorptive action of the two substances, nickel adsorbing hydrogen and alumina water. The rate and character of the reaction undergone by a given reaction-mixture may thus vary with the character of the catalyzer, according to the latter's special adsorbent properties.

It has been mentioned that simple adsorption is insufficient to account for the great activity of certain contact catalyzers. Taylor ${ }^{2}$ points out that charcoal adsorbs carbon monoxide and oxygen but does not catalyze the reaction; but in the case of ethylene and oxygen it both adsorbs and catalyzes; hence as catalyzer, it differentiates between carbon monoxide and ethylene, although adsorbing both. Metallic oxides, on the contrary, catalyze the oxidation of carbon monoxide. It would appear, therefore, that specific, presumably chemical, relationships enter even in such simple cases.

IBancroft, Journal of Physical Chemistry, XXI (1917), 573; Applied Colloid Chemistry, pp. $40 \mathrm{ff}$.

${ }^{2}$ Hugh S. Taylor, Trans. Amer. Electrochem. Soc., XXXVI (1919), I 50 . 
Facts like these (the so-called "preferential catalysis") may be regarded as evidence of conditions which in their higher developments in living organisms appear as the highly selective specificity of many enzymes.

The recent work of Langmuir and Harkins ${ }^{\mathrm{I}}$ has shown that molecules assume definite orientations at the surfaces at which they are adsorbed. This orientation is undoubtedly a factor in the chemical effect produced; reactive groups may thus be brought into a position in which they more readily come into contact with other molecules (or the reactive groups of other molecules), and in this manner reaction is furthered. Cases of preferential catalysis may thus be explained. The catalytic effectiveness of phase-boundaries exhibits itself in many remarkable ways. Taylor and Langmuir ${ }^{2}$ cite Faraday's observation that a perfect crystal of sodium sulphate does not effloresce until its surface is scratched or broken, when the efflorescence spreads from the injured part over the rest of the crystal. Other similar examples are well known; apparently the reactivity of molecules is altered by the adjacent molecules of reaction-product: the use of "catalystpromoters" in chemical processes illustrates the same phenomenon. Enhanced reactivity at interfaces is in fact a very general phenomenon; and in living matter, with its polyphasic constitution, the conditions are exceptionally favorable for this type of influence.

¿Langmuir, loc. cit.; Harkins, loc. cit.

${ }^{2}$ H. S. Taylor, "Catalysis and Catalytic Agents in Chemical Processes," Jour. Franklin Inst., CXCIV (1922), I; Langmuir, "Chemical Reactions at Surfaces," Trans. Faraday Soc., XVII (I92I), Part III (September); reprinted in Gen. Elec. Rev., XXV (I922), 445. 
It is probably significant that the substances which effect the greatest variety of contact catalyses, carbon and the metals, especially platinum, belong in the class of metallic conductors. ${ }^{\mathrm{I}}$ In such conductors, according to the electron theory, there is ready transfer of electrons from atom to atom, hence their electrical conductivity, and other properties correlated with this peculiarity (optical, etc.). It might be expected that such substances would also facilitate the transfer of electrons to or from molecules with which they are in contact, and thus furnish the conditions necessary for chemical reaction. In other words, factors characteristic of the metallic state may enter in contact-catalysis; for example, the formation of local electrical circuits between different parts of the metallic surface, or oscillation phenomena of a frequency corresponding with that of the combination-electrons of the interacting substances (resonator effects). An example of the former type of influence was seen in the simple experiment described above, in which the contact of a nobler metal or carbon accelerates or "catalyzes" the formation of ferricyanide filaments from zinc. Combinations of two contact catalyzers seem often to be more effective than either one alone; thus Shenstone found that "platinized charcoal" was extremely effective in oxidizing alcohol, "converting spirits of wine into vinegar in a few hours," and other cases of a similar kind are described by Bancroft in a recent review. ${ }^{2}$ Presumably the two components differ in their potential-difference against the medium,

${ }^{x}$ Contrast, e.g., the lack of catalytic power in collowidal cilica icf. Taylor, Trans. Amer. Electrochcm. Soc., op. cit., p. 150).

${ }^{2}$ Journal of Physical Chemistry, XXI (1917), 04.4; cf. pp. 667 ff. 
and thus local circuits arise which have chemical effects. Rideal and Taylor also describe cases where metallic couples have a greater catalytic effect than either metal singly. ${ }^{I}$ The readiness with which the metallic phase conducts electricity enables any local inequalities in the metal, or in the nature or concentration of materials (e.g., salts and oxidizable and reducible compounds) present in the solution, to give rise to electric currents between different parts of the surface. These local currents may secondarily influence chemical reactions, as in other cases of electrolysis at metallic electrodes. Effects of the reverse or anticatalytic kind may result when the metallic surface is altered in such a way as to lessen the local potential-differences or increase the resistance of the local circuits; strongly adsorbed organic compounds may have both of these effects, as already pointed out.

Electrochemical oxidations at platinum anodes are subject to variations (partly mentioned above) which are possibly referable to the existence of local circuits; these exercise their own influence independently of the E.M.F. applied from without, and give rise to interference and summation phenomena of various kinds. The possibility that the formation of local circuits plays a part in the normal catalytic activity of platinum and other metals in liquid systems seems to have been insufficiently considered by chemists. The essential conditions of such catalysis are perhaps best illustrated by the decomposition of hydrogen peroxide, which is effected by a large number of finely divided metals of which platinum is especially active. The striking

${ }^{x}$ Rideal and Taylor, Catalysis in Theory and Practice (Macmillan, I9I9), pp. I30, I6o. 
parallels between this action and the similar action of organic fluids and cell-extracts have long been known. The decomposition of $\mathrm{H}_{2} \mathrm{O}_{2}$ by living tissues is now regarded as due to a special enzyme, catalase. The relation of this catalytic action of tissue-extracts to enzyme action and fermentation was early recognized by Schönbein, who characterized the splitting of $\mathrm{H}_{2} \mathrm{O}_{2}$ as a model or prototype of all fermentative processes (Urbild aller Gährungen). ${ }^{x}$ A study of the conditions under which it occurs in the presence of metals may thus throw some light on the general nature of catalytic effects in heterogeneous systems and especially in living protoplasm.

Among other metals mercury shows great activity in decomposing $\mathrm{H}_{2} \mathrm{O}_{2}$. The most striking feature of the catalytic decomposition of $\mathrm{H}_{2} \mathrm{O}_{2}$ in contact with a mercury surface is that under certain conditions the process exhibits a definite regular rhythm, closely resembling physiological rhythms like that of the heart beat in its frequency, in its dependence on temperature $\left(\mathrm{Q}_{x_{0}}=\right.$ about 2), and in the influence exerted upon it by chemical and electrical conditions. This phenomenon has lately been the subject of much investigation, specially by Bredig and his students, and its detailed conditions have been studied most closely by Antropoff. ${ }^{2}$ The chief result of this study has been to show that the catalytic rhythm is dependent on the alternate formation and dissolution of a surface-film of oxidation-product ("peroxidate") formed by interaction between the mercury and the peroxide. Since evidence from various sides indicates

s Schoenbein, J. prakt. Chem., LXXXIX (1863), $335^{\circ}$.

${ }^{2}$ Antropoff, Z. physik. Chem., LXII (1907), 513. 
that many vital processes (stimulation, cell-division) are also dependent on changes undergone by surfacefilms - those forming the plasma membranes and other protoplasmic partitions - this feature of the $\mathrm{H}_{2} \mathrm{O}_{2}$ catalysis suggests that the foregoing physiological parallel may be more than a superficial one, and that the similarities depend on a fundamental identity in the conditions controlling the course of the reactions in the two systems. It is necessary therefore to examine more closely into the nature of the conditions controlling the activity of the $\mathrm{Hg}-\mathrm{H}_{2} \mathrm{O}_{2}$ system.

The rhythm is best shown in Io per cent aqueous solutions of $\mathrm{H}_{2} \mathrm{O}_{2}$. The film which forms over the surface of pure mercury in this solution is gold-brown in color, and the conditions for the rhythmical reaction are best when the solution is slightly on the alkaline side of neutrality. The evolution of oxygen occurs during the breaking down of the film; when the film covers the entire surface of the mercury the reaction ceases. If the solution is slightly acid, the film is stabilized and no evolution of gas occurs; at the appropriate degree of alkalinity it is alternately formed and broken down with a regular rhythm of about ten to fifteen per minute $\left(\right.$ at $\left.I 8^{\circ}\right)$. The film shows great sensitivity to the presence of foreign substances, including salts and surface-active organic compounds (such as ether and olive oil); the latter abolish the rhythm. ${ }^{\mathrm{I}}$ It is also highly susceptible to changes in electrical polarization. Many other striking parallels with physiological rhythms are described in the article by Bredig and Wilke. ${ }^{2}$

${ }^{2}$ Cf. Bredig and Weinmayr, Z. physik. Chem., XLII (1903), 6or.

${ }^{2}$ Biochem. Zeitschrift, XI (1908), 67. 
The fact indicating most clearly the essential nature of the conditions governing the course of the reaction is that the rhythm of decomposition is associated with a parallel rhythm of electrical potential. The mercury in contact with the $\mathrm{H}_{2} \mathrm{O}_{2}$ solution is always found cathodal with reference to the calomel electrode, but during the active phase, while $\mathrm{O}_{2}$ is being freed, it is less so than during rest; i.e., the metal becomes anodal relatively to the resting condition. According to Antropoff's measurements, the inactive mercury is about 0.12 volt more cathodal (i.e., nobler) than the active. This difference resembles that between passive and active iron in nitric acid solution, the inactive mercury corresponding to the passive iron. A change of surface-tension accompanies the change of potential, the rounded convex surface of the mercury becoming flatter (from decreased surface-tension) as the film forms; this behavior is in accordance with the LippmannHelmholtz rule of electrocapillarity, according to which the surface-tension decreases with increase of the potential-difference across the surface. Graphic registration of the curve of potential change shows that its course runs closely parallel with the curve of oxygen evolution as measured by a manometer.

Close observation shows that the gas is erolved only during those times when part of the mercury is filmcovered and part bare; further, that the evolution of gas occurs chiefly near the boundary between the bright and the film-covered surfaces. When a regular rhythm is established, the reaction during each cycle is observed to pass rapidly over the surface of the mercury in a wavelike fashion. At the beginning of a cycle, when 
the whole mercury surface is film-covered and inactive and is flattening as a result of the lowering of surfacetension, a rupture of the film appears, usually at the margin of the drop, disclosing the bright metallic surface beneath. Instantly an effervescence starts at the edges of this fissure and then sweeps over the whole surface of the metal; a new film is then formed and the cycle is repeated. In an inactive drop, an artificial mechanical rupture will often initiate a reaction which similarly spreads rapidly over the whole surface.

Bredig found that a stationary film formed over an inactive surface of mercury is quickly dissolved by rendering the metal cathode; ${ }^{\mathrm{T}}$ in this process of dissolution oxygen is freed. The essential condition of the transmission thus becomes clear. When a film-covered and a bright area of the mercury surface adjoin each other, e.g., after a local rupture of the film, a local electrical circuit is formed between the two, the film-covered area being the cathode of the local circuit. The current of this circuit has the effect of dissolving the film, by cathodic reduction, for a certain distance (estimated at I-3 millimeters) from the boundary, oxygen being freed in the process; and by repetition of this effect at each new boundary as soon as it is formed the effect spreads rapidly over the whole surface. The $\mathrm{Hg}-\mathrm{H}_{2} \mathrm{O}_{2}$ pulsating catalysis thus in reality represents an intermittent electrolysis of $\mathrm{H}_{2} \mathrm{O}_{2}$ under the influence of the temporary local circuits formed during the alteration or removal of the surface-film.

Rhythmical chemical processes at the surfaces of metals immersed in electrolyte solutions containing ${ }^{3} \mathrm{Cf}$. Bredig and Wilke, op. cit., p. 69. 
compounds which interact with the metal are not infrequent; e.g., when the metal is undergoing solution in a strongly oxidizing acid like $\mathrm{HNO}_{3}$. Iron in particular often illustrates this phenomenon with great beauty and regularity; in this case the essential condition of the rhythm is an alternation between active and passive states, due, as in the mercury catalysis, to the alternate formation and dissolution of a protective surface-film of oxidation-product. All of these inorganic rhythms are highly susceptible to variations in external conditions, and especially to electrical influences.

The rhythmical processes so frequent in living organisms (rhythms of cilia, muscle, nerve cells, vacuoles, and cell-division) show many close parallels with these inorganic "surface-reaction" rhythms. As in metals, they are associated with rhythmical variations of electrical potential and with rhythms of chemical or metabolic alteration and surface-change (clearly demonstrable, e.g., in cell-division), and are similarly susceptible to changes in the surrounding conditions (temperature, H-ion concentration, presence of salts and surface-active compounds, electrical polarization, etc.). These parallels imply a similarity in the essential determining conditions in the living and the non-living systems. Since rhythmical catalysis in metals is dependent on the polyphasic character of the system - this being the condition which makes possible rapid local variations of potential, resulting from changes in the composition and structure of surface-films - the hypothesis that the organic rhythms are similarly conditioned naturally suggests itself, particularly when the film-pervaded or emulsion-like

${ }^{x}$ For earlier observations cf. Bredig and Wienmayr, loc. cit. 
structure of living matter and the various facts showing the dependence of stimulation on membrane processes are taken into consideration. The general susceptibility of living matter to electrical influence suggests that in protoplasm there may be a similar dependence of the chemical reactions upon processes of electrolysis occurring at the boundaries between the protoplasmic phases. This general interpretation is also consistent with the readiness and rapidity with which chemical influence is transmitted from region to region in living matter; the many close resemblances between such transmissions and the transmission of the waves of electro-chemical alteration over the surface of mercury or passive iron will be considered later in detail. In these inorganic systems the chemical reactions are directly determined by the potential-differences existing between different portions of the metallic surface; these potential-differences arise as the result of local alterations of the surface-films, and the local circuits thus arising effect the chemical change by electrolysis. Similarly in living matter the waves of chemical and physiological alteration accompanying the transmission of stimulation (i.e., excitation-waves, nerveimpulses, etc.) are always associated with waves of electromotor variation. Bernstein first showed for motor nerves (in I866) that the physiological effect and the bioelectric variation have the same velocity of propagation; ${ }^{\prime}$ and all of the more recent evidence confirms the view that the electric variation is the essential component of the transmitted process.

Recently I have discussed in some detail the parallels between the transmission of chemical effects in systems

Cf. Bernstein's Elektrobiologie, chap. iii, for references. 
consisting of metals immersed in electrolyte solutions and the transmission of physiological influence in living protoplasm. ${ }^{\text {I }}$ In all such phenomena in metals the essential condition is the presence of a thin film of electrochemically alterable material formed or deposited at the interface between the metal and the electrolyte solution. Local circuits between adjoining regions of the film-covered surface, differing in composition or physical condition in such a manner as to give rise to an E.M.F. sufficient for electrolysis, are in all cases the essential factor. By the action of these local currents the film is locally altered or removed or rendered permeable

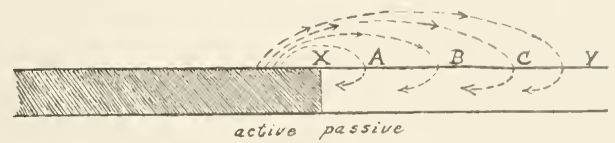

FIG. 3.-Indicating the conditions of the local circuit at the boundary between the active and the passive areas of an iron wire in nitric acid; the direction of the current (positive stream) is indicated by the arrows, the active region (shaded) being anodal, the passive cathodal. The local intensity of the current in the passive region (and hence the reducing or activating effectiveness) decreases in the order $A<B<C$; beyond a certain distance from the boundary, e.g., $X Y$, it will be insufficient to activate.

over a certain area (usually cathodal, e.g., XY, Fig. 3), adjoining the boundary between the two regions, and the similar circuit which is then formed at the boundary between the newly altered area and the unaltered area beyond repeats the effect; hence the alteration automatically spreads over the whole surface. A wave of such transmission is necessarily associated with a wave of electromotor variation.

A brief account of the phenomena of activation and transmission in passive iron will indicate more clearly

'American Journal of Physiology, XLI (1916), 126; Science, XLV'III (1918), 51; L (1919), 259, 416; Journal of Physical Chemistry, XXIV (1920), 165; Jour. Gen. Physiol., III (1920), 107, I 29. 


\section{PROTOPLASMIC ACTION AND NERVOUS ACTION}

the general nature of such processes. ${ }^{{ }^{x}}$ Passivity is readily induced in an iron wire by immersion in strong nitric acid (sp. gr. I.42); the metal then remains unaltered or chemically inactive when transferred to weak acid (sp. gr. I.2), unless it is artificially "activated." Activation may be induced by various means, chemical, mechanical, and electrical. The following simple and readily performed experiments will bring out clearly the chief resemblances between the processes of activation and transmission in the metallic systems and in living protoplasm.

When the passive iron wire is immersed in a dish of nitric acid of about 60 volumes per cent concentration (i.e., of commercial $\mathrm{HNO}_{3}$ of sp. gr. I.42), no change occurs, and the surface of the metal remains bright and unaltered. If, however, it is then touched with a piece of ordinary "active" iron, or with a base metal like zinc, a local reaction, accompanied by effervescence and a darkening of the bright metallic surface, is at once initiated and sweeps rapidly over the whole wire from end to end. In acid of the foregoing concentration, the local reaction ceases in one or two seconds (at $20^{\circ}$ ), and the metal reverts automatically to the passive state. Immediately after this repassivation it is resistant to activation and transmits the reaction imperfectly; on standing, transmissivity gradually returns and within a minute is usually again complete; the metal can then be activated as before and the same phenomenon is repeated. The passive wire may be activated mechani-

${ }^{x}$ For a general review of the phenomena of passivity in metals cf. Bennett and Burnham, Journal of Physical Chemistry, XXI (1917), I07. 
cally by jarring or bending or by scraping with a piece of glass; summation effects are a conspicuous future of this form of activation; a single scrape or blow, or a succession of these at infrequent intervals, being usually ineffective, while several scrapes in rapid succession cause typical activation. Chemical activation may be shown by the application of a reducing agent like sugar. The same kind of effect is produced in the wire, whatever the method of activation, the local change simply.initiating a propagated effect whose nature and extent depend on the special conditions existing in the metal-electrolyte system. There is here an evident analogy with explosions or other kinds of "trigger effects." Hence the system, when in a fully transmissive state, behaves in the "all-or-none" manner.

In reality electrical activation is illustrated in all these cases, even when the local alteration initiating the reaction is mechanical or chemical; i.e., the electrical factor is the essential one in the transmission of the effect. The special conditions of electrical activation are, however, best shown by a somewhat different kind of experimental arrangement. Two passive wires, placed parallel one or two centimeters apart, are immersed in a vessel containing dilute $\mathrm{HNO}_{3}$ and are connected by wires and an open key to a battery (e.g., of about 2 volts E.M.F.). When the key is closed, the cathodal wire (that connected with the negative pole or zinc of the battery) is at once activated, while the anodal wire remains unchanged. This experiment shows that activation is a polar effect and dependent on cathodal reduction. Activation also requires a certain minimal E.M.F. in the battery, usually exceeding one volt, and a certain minimal 
duration of flow of the current. Electrical summationeffects similar to those of mechanical activation can also be demonstrated under appropriate conditions. ${ }^{\text {I }}$

Another influence of the current is especially interesting from its resemblance to the physiological phenomenon of electrotonus; this consists in a modification of the susceptibility of the wire to mechanical or other activation. During the flow of the current the automatic return of passivity in the active (cathodal) wire is delayed, or with sufficient strength of current prevented, and the anodal wire becomes more resistant to mechanical or other activation. If with two passive wires in a circuit, as above, a constant current too weak to cause activation under these conditions (e.g., the current from one Edison cell) be passed, the cathodal wire, although remaining passive, is rendered temporarily more susceptible than before to activation by other means, e.g., mechanical treatment, while the anodal wire becomes less susceptible. ${ }^{2}$

All of the foregoing phenomena have their parallels in the behavior of living irritable tissues under the influence of the electric current; the corresponding physiological phenomena are summation, polar stimulation, chronaxie, enhancement of irritability near cathode, and its decrease near anode ("electrotonus").

Phenomena of a similar kind are seen in the mercuryperoxide system; here also the inactive mercury may be activated by making it the cathode in a circuit, or

${ }^{x}$ E.g., by using the brief contact of a copper wire as an activating agent. The local current thus produced may be too brief for activation by a single contact, while several contacts in close succession will produce the effect.

${ }^{2}$ For a somewhat fuller description cf. Jour. Gen. Physiol., III (I920), I36. 
the rhythm of an automatic pulsation may be altered. During the flow of the current the catalytic effect at the cathode is heightened, while at the anode it is decreased or the rhythm may be abolished. ${ }^{x}$ The parallelism between these effects and those produced by the constant current on the action of rhythmical tissues like the heart is evident.

A more detailed account of the phenomena in filmcovered metallic systems of this kind is not possible within the limits of space, but attention should be called to another interesting feature of the electrical initiation of these reactions. A peculiarity of the electrical activation of passive iron is that it is not readily produced by currents which rise slowly from a minimal strength to a strength sufficient to activate with sudden closure. ${ }^{2}$ In other words, the rate of change of the activating current is an essential factor in the effect produced. This is a well-known and highly characteristic feature in the response of living tissues to electrical activation. Each tissue has its characteristic time-factor of electrical excitation or so-called "chronaxie," and this is closely related to the rate of change required of a stimulating current (cf. p. 288). The time-relations of the activating current in the metallic system resemble those in living tissues in the further respect that in the case of alternating currents the relation between the intensity required for activation and the rate of alternation follows the same law, as shown by Bredig and Kerb for the mercury $\mathrm{H}_{2} \mathrm{O}_{2}$ system; 3 that is, there is an inverse relation be-

${ }^{x}$ Bredig and Wilke, Biochcm. Zeitschrift, XI (1908), 67.

${ }^{2}$ Science, XLVIII (1918), 57 .

3 Bredig and Kerb, Verh. nalurhislorisch-med. Vercins su Heidel. berg, X (1909), N.F., 23. 
tween the intensity required to activate and the root of the number of alterations $(i / \sqrt{n}=$ const. $)$. This relation has been shown by Nernst ${ }^{\mathrm{I}}$ and others to be generally characteristic of electrical excitation in living tissues; it indicates that a current of a given intensity must flow for a certain minimal time in one direction through the irritable system in order to cause activation. I.e., the change in the electrical polarization of the surface concerned in activation must last for more than a certain critical time, presumably the time necessary to produce a certain critical degree of chemical change. The physical conditions of response to electrical influence thus appear to be of the same kind in the living system and in the inorganic model.

${ }^{2}$ Nernst, Arch. ges. Physiol., CXII (1908), 275; cf. chap. xii. 


\section{CHAPTER XII}

\section{STIMULATION AND TRANSMISSION OF EXCITATION IN PROTOPLASM}

Responsiveness to stimulation is a universal characteristic of living matter. Typically the reaction to a stimulus involves a performance of work (i.e., transformation of energy) which has no definable proportion to the work done by the stimulating agent upon the living system. The stimulus usually acts locally, yet the whole living system-cell, tissue, or even entire organism-may be thrown into activity. Transmission of physiological influence from the immediate site of stimulation to other regions of the living system is thus a constant feature of stimulation. Hence the subject of the essential conditions determining this transmission is one of fundamental biological interest; evidently the living system can react as a whole, i.e., in a unified or correlated manner, only in so far as the physiological processes in any single region occur in correlation with those in other regions. This transmissive property of protoplasm is the primary integrative factor in organisms; its highest development has been attained in the nervous system of higher animals. ${ }^{\mathrm{x}}$

In general, local variations in protoplasmic activity, implying variations in the rate or character of the underlying chemical or metabolic processes, influence other processes occurring at a distance from the active

${ }^{x}$ Cf. my review of the subject of protoplasmic transmission in Physiological Reviews, II (1922), I. 
region. The physical constitution of the living substance is evidently of such a nature as to permit rapid transmission of chemical influence to a distance; in other words, some form of "chemical distance-action" is a constant feature of protoplasmic action. It is natural to connect this feature with the essential or fundamental features of the physical structure of protoplasm. We have already seen that this structure is polyphasic and film-pervaded, or emulsion-like. It is therefore highly interesting to note that the inorganic transmissive processes just considered, which bear such a striking resemblance to the transmissive processes of protoplasm, are in fact determined by chemical and structural alterations in thin surface-films, and that these alterations occur under the influence of local electric circuits. In such a system as passive iron in nitric acid the chemically reactive material whose alteration determines the transmission is spread out in a thin layer or film at an interface (metal-electrolyte) which is the seat of a potential difference. The surface of contact of this material with the adjacent layer of electrolyte solution is a large one, relatively to the total mass of reacting substance. This arrangement makes for a rapidly acting and sensitive type of reaction-system, since the removal or alteration of a very small quantity of material may, by altering electromotor conditions at the surface, form the condition for a spread of chemical effect, electrically conditioned, which may be very extensive and rapid. ${ }^{x}$ Transmission depends on the instantaneous

${ }^{x}$ It may be pointed out here that the importance of extremely small quantities of certain special substances, e.g., vitamines in animals, is probably a correlative of the control of chemical reactions in protoplasm 
passage of an electric current through the circuit constituted by the two chemically or structurally different portions of this thin interfacial film, together with the electrically conducting phases (in this case metal and nitric acid) between which it is interposed. In living protoplasm, with its film-partitioned constitution, it seems probable that the structural arrangement or disposition of the chemically reactive material which determines the response to stimulation is of a similar kind; i.e., that this material is disposed in the form of a thin film between two electrically conducting phases, a type of arrangement allowing transmissions to occur under conditions of essentially the same physical kind as in the foregoing inorganic type of system.

It has already been pointed out that stimulation processes cannot be considered separately from the processes of transmission or conduction. In general the effects of local alteration in protoplasm tend to spread: i.e., to produce chemical and physiological effects in other regions than those immediately acted upon by the stimulating agent. In some cases this spread is limited in extent; but in others, especially nerve, there appears to be no limit to the distance through which the change of activity may be transmitted. Hence the total effect of any stimulation has no fixed relation, quantitative or qualitative, to the direct physical effect produced by the stimulus at its point of application. In many

by film-structure. When material is in a film, small quantities may determine large chemical effects, because under these conditions what is important is not so much the quantity of material as the area which it covers. Surface relations rather than mass or volume relations then become the controlling factor. 
irritable systems with highly developed transmissive properties, e.g., the nerve fibers and muscle cells of higher organisms, the character and intensity of the response are quite independent of those of the stimulus, provided the latter attains the threshold value. A full response, involving the whole irritable element, results from either a "weak" or a "strong" stimulus; this is the "all or none" type of behavior, which is found also in many physical systems in unstable equilibrium, and also in explosive systems or others in which chemical change is rapidly transmitted; e.g., the passive iron system. In all such cases there is a "release" of stored energy, and the work performed by the releasing agent has no definite relation to the energy transformed in the resulting process. ${ }^{x}$

The phenomena of stimulation in living organisms are so various that one hesitates to regard them all as determined by conditions of the same physico-chemical kind. Nevertheless, it is a striking fact that whatever the special peculiarities of the organic activity or response in different living systems may be, the conditions of initiation and control are remarkably uniform. The universal susceptibility to the electric current, to mechanical disturbance, and to certain kinds of chemical influence, especially the influence of inorganic salts and the lipoid-solvent or surface-active group of organic compounds, indicates that the fundamental structural and chemical conditions underlying the response to stimulation are the same in all forms of protoplasm. It is

"The typical case is one of "trigger action," which is a characteristic feature of all modes of organic response (cf. the interesting discussion of Lotka: "Natural Selection as a Physical Principle," Proceedings of the National Academy of Science, VIII [I922], 15I). 
especially to be noted that the two of the most general features of stimulation-processes, viz., the susceptibility to the electric current, and the reversible modification or suppression of irritability by the surface-active groups of compounds (aniesthesia or narcosis), indicate definitely a dependence of protoplasmic activity on the polyphasic structure of the system. The inference from such facts is that the chemical reactions of protoplasm are controlled by the peculiar conditions resident at the protoplasmic interfaces or phase-boundaries; and the resemblance between the conditions of activity of irritable protoplasmic systems and of the inorganic models just described confirms this inference.

Some of the more general features of the phenomena of stimulation in living organisms have already been discussed briefly. Since continued life depends on a regulated interchange of material and energy with the environment, it is to be assumed that all fundamental vital activities are capable of varying in correlation with, or "in response to," environmental change; the character and rate of the interaction of the living system with its environment are thus controlled. Normally the responses of any organism to stimulation are of such a kind as to favor its continued or stable existence in this environment. A certain difficulty in defining the conception of stimulation arises here, since many cases exist where physiological activities, which in themselves are injurious or destructive to the living system as a whole, may be induced by environmental change; ${ }^{\text {such }}$

${ }^{I}$ The oxidation rate of sea-urchin eggs may be increased by pure $\mathrm{NaCl}$ solution to a degree which apparently is directly destructive. The case of fatigue carried to an injurious extreme is analogous. 
instances would scarcely be classed as responses to stimulation. In fact many opportunities for verbal mystification arise in attempting to "define" the concept "stimulation." In order to limit the following discussion, we shall regard as a "stimulus" any influence acting from without upon the living system which changes the rate or the character of the normal vital activities; the resulting change of physiological activity is the "response." Even with this simplified conception, the range of phenomena is still too great to be readily included under any strictly drawn definition; but such a definition need not be insisted upon, provided the general nature of the relations between organism and environment is clearly understood. Variation of vital activity, within the physiological range, occurring as a constant correlative or sequence of environmental change of some kind, is the essential phenomenon whose conditions we are considering.

In multicellular organisms, "internal" and "external" (proprioceptive and exteroceptive) stimuli are often distinguished, ${ }^{x}$ since in many cases the environment which furnishes the normal stimuli for an irritable cell or cell-system may be not the external world but some other part of the same organism. Responses of special organs or organ-systems to stimuli originating elsewhere within the same organism form, in fact, a regular part of many normal physiological cycles in higher animals; thus the pancreas is stimulated by secretin in the blood stream, and the respiratory center by increased $\mathrm{H}$-ion concentration of the blood; the central nervous system is continually adjusting its

${ }^{x}$ Cf. Sherrington, Integrative Action of the Nervous System. 
activity to changes in bodily conditions, and so on. Evidently the effects of external stimuli upon the sense organs cannot be regarded as forming a sirnificantly different class from these phenomena, so that from the standpoint of general physiology the foregoing distinction is a purely formal one and has little objective importance.

Various terms are applied to special processes which may be included under the general conception of stimulation, as just defined. The term "activation" is userl with reference to the initiation of development in a resting egg cell by a spermatozoön or a parthenogenetic agent; acceleration, or simple increase in the rate of an already existing process, is a frequent form of response (e.g., secretion, the heart-beat, or other regular muscular movement, growth, etc.); retardation or inhibition is perhaps equally frequent. In cases of automatism, like that of the heart, the rhythm may be regarded as determined by periodic stimuli furnished by processes within the cell. Since all of these phenomena may occur, or undergo modification, in response to changes of environmental condition, all are to be considered under the general conception of stimulation.

The most general features of the stimulation-process are best studied in those irritable tissues or cells which give a prompt and definite response to clectrical or mechanical stimulation, such as the nerves and muscles of higher animals; and the majority of investigations. on stimulation, especially those of a quantitative kind. have been carried qut with these tissues, usually after isolation. The results gained have, however, a generil applicability to other irritable living systems. 


\section{GENERAL CONDITIONS OF STIMULATION}

It is well known that different living systems may vary widely in their sensitivity to the same stimuli, and also that irritability is often specialized with reference to particular physical agents. Sensory elements with special sensitivity to light, contact, slight changes of temperature, or chemical substances, are found in all higher animals. These differences are referred to special features of chemical and structural organization. Chemical sensitivity in particular is often minutely specialized; and such instances as the special sensitivity exhibited by the sensitized smooth muscle of guinea-pigs in anaphylaxis indicate clearly that many forms of specific chemical irritability are dependent on the presence of specific chemical compounds (apparently in this case proteins) in the irritable cell, probably in the protoplasmic surface layer. Similarly, photo-sensitive elements like the retinal rods and cones contain compounds of definite photochemical properties (visual purple and related substances) upon which the special responsiveness undoubtedly depends. ${ }^{\mathrm{I}}$ We must recognize, therefore, in addition to the general susceptibility to mechanical or electrical stimuli possessed by all forms of protoplasm, a variety of specific or selective forms of irritability depending on special features of structure or organization. Selective irritability is shown especially by the sensory nerve-termini or receptors of higher animals; these are classified, according to the agents to

I Thus Hecht and Williams have recently shown that the curve of absorption of visual purple is almost identical with the curve of visual sensitivity for different wave-lengths (Jour. Gen. Physiol., IV [1922], r). 
which they are specially responsive, as chemo-receptors, thermo-receptors, photo-receptors, etc.

Such special sensitivity may be described as consisting in a lowering of the threshold of stimulation for a particular agent, and need not affect the general sensitivity to mechanical and electrical stimulation. Some specific irritability is superposed upon the general or nonspecific irritability. Thus a nerve or muscle may be stimulated by mechanical, thermal, chemical, osmotic, and electrical stimuli; similarly, a highly specialized receptor such as a retinal element may be stimulated by these agents as well as by light of a definite wavelength. In all cases, however, the response following stimulation has a specific character which is dependent on the special structure or organization of the irritable system or on its relations with other systems. In the field of sensory stimulation this generalization is known as the "law of specific energies."

LOCAL CHANGE AND PROPAGATED EFFECT

We have seen that an irritable system with a highly developed general sensitivity, e.g., a muscle or a nerve, may be excited by a variety of stimulating agents, and the question arises why such physically dissimilar agents produce the same physiological effect. The general sequence of events when such a tissue is stimulated may be briefly described as follows. Some local change, whose precise nature is determined by the nature of the stimulating agent, occurs at the site of stimulation; a state of "excitation" is there initiated which, however, does not remain confined to this region, but spreads or is propagated to a distance, often at a high velocity. 
This propagated effect, or "propagated disturbance" (Keith Lucas' term), as it appears at a distance from the point of stimulation, has its own definite peculiarities, which are independent of the nature of the initiatory local change or stimulus. For example, any single nerveimpulse in a normal frog's nerve, by whatever means it is initiated, travels at a constant velocity (assuming the state of the tissue to be normal and the temperature and surrounding conditions constant), and its most readily observed physical accompaniment, the bioelectric variation, has a definite range of potential change and definite time-relations. In other words, the propagated disturbance differs from the local change in exhibiting constant and specific features, qualitative and quantitative, whose nature is determined by the special or inherited constitution of the tissue.

Almost any kind of sufficiently rapid local alteration may initiate such a wave of physical and chemical disturbance. The distinction between the local change and the propagated effect is a fundamental one in any theory of stimulation. The former is the "releasing" event and follows upon some simple physical change produced in the tissue by the stimulating agent; the latter is the distinctively physiological process, and as such has specific characters of a complex kind, dependent on the nature of the irritable system and as yet imperfectly analyzed.

Especially significant is the fact that all irritable elements, apparently without exception, respond to electrical stimuli, or are influenced in their already existing activity by the electric current. The electrical sensitivity of highly irritable tissues, such as vertebrate 
motor nerve, is extreme; the frog's sciatic nerve may be stimulated by a current of .ooooor ampere or less: it is well known that the neuro-muscular apparatus of the frog was used by Galvani as the most sensitive means known to him by detecting variations in the electrical state of bodies; in fact it is to this property of living tissues that we owe the discovery of current electricity by Volta. Galvani's experiments also showed -although their significance was disputed at the timethat electric currents are produced in the activity of living tissues. Thus two of the most fundamental properties of living matter, its electrical sensitivity, and its production of electrical currents during activity, were early observed; and many of the chief problems of general physiology at the present time relate to the physico-chemical conditions and physiological significance of these properties. That they are among the chief factors controlling normal cell-processes seems certain.

\section{NATURE OF THE LOCAL CHAYGE}

It is remarkable that complete stimulation, involving a change in the activity of the entire cell, is producer in many if not all irritable elements by agents which affect directly only the surface-layer of protoplasm. Some local modification of surface conditions secms to be all that is required to set in motion the whole complex process of stimulation. This is best shown in the mechanical stimulation of single cells; thus in a ciliated protozoön like Paramecium a slight touch is sufficient to call forth the characteristic motor reaction, involving a reversal of the direction of ciliary activity over the whole surface of the organism. The extraordinary 
sensitivity of many blood cells to mechanical contact illustrates the same phenomenon; a slight touch with a capillary needle is often sufficient to cause a rapid and complete disintegration of a leucocyte or a red blood corpuscle; ; i.e., a wave of alteration involving the breakdown of the semi-permeable surface-film is propagated over the entire protoplasmic surface. The breakdown of the explosive corpuscles in Crustacea ${ }^{2}$ and of nematocytes in cœlenterates are other examples of the same kind of process. Such facts suggest that in normal cases of stimulation, as in nerve, where a local stimulus initiates a temporary disturbance, which passes like a wave over the entire irritable element, a similar disintegration of the surface-protoplasm occurs, with the difference that this change is immediately and rapidly reversed by the formation of a new surface layer.

That some such process occurs during the transmission of the excitation-wave in a nerve or other irritable tissue is indicated by the character of the local bioelectric variation. A reversible surface-change is known to accompany the transmission of the activationwave along a passive iron wire immersed in nitric acid; the passivating surface-film is removed by electrolytic reduction in the neighborhood of each active area, and is then immediately reformed by the oxidizing action of the acid and of the local electric current (at the anodal areas); and the destruction and re-formation of the film are associated with definite and rapid variations of potential. In the stimulated living system a similar

${ }^{x}$ Cf. Chambers, Anatomical Record, X (1916), I9o.

${ }^{2}$ Cf. Hardy, Journal of Physiology, XIII (1892), I65; Tait, Quarterly Journal of Experimental Physiology, XII (I918), 42. 
reversible variation of potential accompanies the passage of the excitation-wave. The resemblance of the transmission phenomenon in passive iron to the protoplasmic type of transmission is in fact so detailed as to confirm strongly the hypothesis that in the latter case also the essential feature of the transmission-process is the breakdown and reconstruction of the thin protoplasmic surface-film under the influence of the local bioelectric circuits.

If the processes in the living system and in the simple inorganic model are in fact similar in their dependence on surface-changes of this kind, it becomes evident at once why protoplasm is so readily excited by conditions acting upon its surface layer. A mechanical agent, by interrupting the continuity of the surface-film, or by otherwise altering it so as to give rise to a local circuit, may initiate a wave of electromotor variation and disintegration which travels automatically over the whole surface, and in so doing alters the physiological activity of the whole system. Any other sufficient local alteration (chemical, thermal, etc.) may produce the same effect. The critical factor in the local process of excitation thus appears to be the alteration of the protoplasmic surface-film in such a manner as to change locally the potential difference between the protoplasm and the external medium, to a sufficient degree and at a suficient rate. The local circuit arising between this altered region and the as yet unaltered regions adjoining forms the next link in the chain of events; and if this local current has sufficient intensity and local density to break down electrolytically the film over a certain area of the adjoining region, an indefinite wave of propagation 
may be initiated, since the same effect will be repeated at each newly formed boundary between the active and the inactive areas.

According to this conception the primary action of a local stimulating agent is to change rapidly the electrical condition of the cell-surface at its point of application. The parallel between the irritable protoplasmic system and the passive iron model is obvious, since in the latter case also a local alteration of the surface-film involving a local change of potential is the initiating condition for the propagated wave of chemical and electromotor disturbance.

The present view, therefore, regards stimulation as conditioned by surface processes of the foregoing definite kind. If this is true, the structural arrangements providing for the transfer of excitation from one irritable element to another should exhibit features of a character to correspond. In fact, many peculiarities of the structure of the central nervous system-especially of the synaptic junctions - and of the structure and arrangement of nerve-endings, such as the myoneural junctions, are in harmony with this conception. Nerve end-plates spread out over the surface of the muscle cell, effecting intimate contact but not penetrating; the junctions between neurones are effected by brushlike interlacing terminals, or by end-feet and similar structures which are applied to the cell surface with a closeness that apparently admits of variation. That transmission by contact, through the influence which the cell-process exerts upon adjoining processes or upon the cell body, is the chief mode of transmission in the nervous system is one of the corollaries of the neurone theory of the 
structure of this system. The well-known experiments ("rheoscopic frog") in which one active muscle or nerve stimulates another which is in close contact with it, by means of the bioelectric currents accompanying activity, show that excitation can be transmitted from one irritable element to another without direct protoplasmic continuity, through a purely electrical influence. Similarly in the passive iron nitric acid system, activation is readily transmitted from one wire to another by contact, and the basis of this transmission is also electrical.

According to these conceptions, all forms of stimulation are electrical; or, more exactly expressed, electric currents resulting from local alterations of the cell surface form a necessary part of the sequence of processes constituting stimulation. Such a view implies further, since the activity of the whole cell is altered by stimulation-e.g., all of the fibrils in a muscle cell contract when the excitation-wave travels over its surface - that the intracellular processes, including the chemical or metabolic processes which furnish the energy for the activity, are largely controlled by processes having their origin at the cell surface.

This inference is confirmed by a study of the conditions of electrical stimulation. The work of Nernst and his successors ${ }^{\mathrm{I}}$ has shown that the electric current does not act by penetrating the living cell (which in fact is a poor conductor) but by changing its surface-

${ }^{x}$ Cf. Nernst, Göllingen Nachrichlcn, math.-phy'sik. Klasse (1\$og). p. 104; Arch. ges. Physiol., CXXII (1908), 275; Lapicque, Jowr. de Physiol, IX (1907), 565, 620; X (1908), 601; XI (1909), 1000), 1035, Lucas, Journal of Physiology, XL (1910), 225; A. V. Hill, ibid., XL (I910), I90. 
polarization. And the semi-permeability of the plasma membranes of irritable cells (e.g., muscle-cells) with reference to the inorganic salts of the medium-which, without penetrating the cell, nevertheless influence profoundly its properties and activity-shows further how closely cellular activities are dependent on surfaceconditions. Among these surface-conditions the state of electrical polarization appears to be of primary importance.

The facts of electrical stimulation, now to be considered, show that variations in electrical surfacepolarization have a far-reaching control over the metabolic and other processes occurring in the cell interior. The means by which this polarization may be altered are of three chief kinds: (I) changes in the structure or composition or permeability of the surface-film; external electrical influences, especially the influence of electric currents traversing the cell or its medium; and (3) changes in the composition of the medium or internal protoplasm. Since, according to the present view, an inseparable feature of stimulation, the transmission of the excitation-state, is a direct result of electrical activation by the currents of local bioelectric circuits, it is clear that the problem of stimulation resolves itself largely into the problem of the general conditions under which the electric current stimulates living matter. Electrical stimulation is in fact the primary form of stimulation.

CONDITIONS OF ELECTRICAL STIMULATION

In general the physiological studies of the last two decades have shown that the stimulation of an irritable 
living system by the constant electric current is subject to definite quantitative laws; they indicate also that the current acts primarily through its polarizing action. Upon this polarizing action follows chemical action as a secondary consequence.

The essential conditions under which the current causes stimulation may be briefly summarized as follows:

I. The current must exceed a certain minimal intensity; this "threshold" intensity varies widely for different irritable tissues and for the same tissue under different conditions; e.g., during states of fatigue, narcosis, sensitization, etc.

2. A current of threshold or greater intensity must traverse the tissue for a certain minimal time; its stimulating action thus depends not only upon its intensity but also upon the duration of its flow. 'The rule that for equal stimulating action the product of the intensity into the root of the duration is constant $(i \sqrt{t}=K)$ appears to hold for most tissues within a considerable range of intensities. ${ }^{x}$ For the current of threshold intensity this critical duration varies widely in different tissues (from a few thousandths to several seconds); it is the expression of a time-factor (chronaxie), which is specific for the tissue in question. ${ }^{2}$

These general statements apply to the case of currents which reach their full intensity rapidly or instantaneously; e.g., when the stimulating circuit is suddenly: closed. Such a condition, however, is apparently not a normal or "physiological" one, since the bioelectric current-which, according to our present conception, is
${ }^{\prime}$ Cf. Nernst, loc. cit.
${ }^{2}$ Cf. Lapicque, loc. cil. (1909). 
the factor arousing the local excitation at each successive region in a transmitting element (nerve, etc.)-attains its full intensity not instantaneously but in a rising curve of more or less gradual slope, varying from tissue to tissue, and subsides in a similar manner. Stimulation by currents of varying intensity is thus the typical condition prevailing in the organism, and to which, therefore, especial attention must be directed. The general empirical rule governing the action of such currents is as follows:

3. In order to stimulate, a current rising continuously and uniformly from zero to full intensity must change its intensity at a certain minimal rate which is characteristic for the tissue; i.e., $\frac{\Delta i}{\Delta t}=$ const. (assuming temperature and other conditions normal). Hence we find that a slowly increasing current may fail to stimulate, while one rising to the same intensity at a more rapid rate stimulates. The same rule applies to stimulation by the decrease of a current already flowing through a tissue. The rate of change, in either direction, must exceed a minimal value which is specific for the tissue. A time-factor enters, closely related to that already referred to above (under 2) as "chronaxie."

4. The stimulating action of the current is characteristically polar; i.e., the current produces its primary physiological effects chiefly at its regions of entrance and exit, and the effects at the two regions are typically opposite or antagonistic. Typically, when the current is made, it initiates excitation at the cathode (i.e., where the positive stream of the stimulating circuit passes from the tissue to the applied electrode), and inhibits 
activity (at the same time depressing irritability) at the anode. When a current already flowing through the tissue is broken, stimulation also results, but the polar relations are reversed; i.e., stimulation is then at the anode, inhibition at the cathode. This summarized statement is the usual form of the "law of polar stimulation." It is important to note, however, that a polar action is seen in many other physiological processes occurring under the influence of the current; c.g., electrotonus, polar disintegration of cells, galvanotropic growth, and galvanotaxis. All of these phenomena show that the direction of the current, relatively to the cell surface, determines the nature of its physiological action. The parallel to electrolysis, at the surface of any electrode. is especially clear in phenomena of this class; it is well known that where the positive stream passes from the metallic electrode to the solution (at the anode) it produces chemical effects (in general of an oxidative kind) of the reverse nature to those produced where it passes from solution to electrode (cathode); here the general chemical action is reducing.

5. That a variation in the electrical state of the irritable elements, sufficient in degree and rate, is the determining factor in the physiological action of the current is seen in the fact that a change in either direction, i.e., make or break, increase or decrease, may stimulate or produce other characteristic physiological effects.

6. Finally, summation effects are highly characteristic; i.e., two or more electric stimuli (induction shocks) which, acting singly, are ineffective, may cause stimulation if sent in sufficiently rapid succession into the tissue. The interval between the successive single stimuli must 
be less than a certain critical time, or no summation results. This "summation time" is closely related to the characteristic time-factor of the tissue, being brief in tissue with brief chronaxie and vice versa. ${ }^{\mathrm{I}}$ Since the stimuli normally acting in the intact organism are largely repetitive or rhythmical, summation processes are of special physiological interest.

An exhaustive discussion of the effects of electricity on living organisms is not possible within the limits of space; but those features of electrical stimulation which indicate its dependence on surface-alteration are of fundamental theoretical significance and will be considered in some detail.

Chief among these features are the time-relations of electrical stimulation. A stimulating current of a given intensity must flow uninterruptedly for more than a certain time through the tissue or it produces no apparent effect. When the relations between the intensity of a stimulating current and its minimal duration are investigated, a highly characteristic relation appears, indicating that the action of the current depends upon the transport of ions to or from the semi-permeable surfaces of the irritable tissue. The resulting change of electrical surface-polarization forms the primary condition of stimulation. This was first clearly shown by Nernst, ${ }^{2}$ in a paper on the relation between the stimulating action of alternating currents and the rate of alternation. In a living tissue, which, considered from a simplified physico-chemical point of view, represents an electrolyte solution partitioned by membranes not readily permeable

${ }^{x}$ Cf. K. Lucas, Journal of Physiology, XXXIX (IgIo), 46r.

${ }^{2}$ Nernst, loc. cit. (I899). 
to ions, there is during the flow of the current a movement of cations with the positive stream and of anions with the negative stream; at the semi-permeable membranes interposed in their path the movement of ions is impeded; the cations then undergo an increase of concentration at those surfaces of the membranes which face towarl the anode, simultaneously with a decrease of concentration at the opposite faces; the reverse relations hold with the anions. A gradient of ionic concentration is thus set up between the layer of solution in immediate contact with the membrane and the layer at some distance. This process of concentration at the semi-permeable surface continues until a condition of equilibrium is reached at which the rate of diffusion back from the membrane into the interior of the solution is equal to the rate at which the ions are transported to the membrane. Assuming the existence of these two opposed processes, transport to the surface by current and backdiffusion, it can be shown that to produce a definite change in concentration at the surface, the product of the current-intensity into the root of its time of flow should be constant $\left(i_{!}, \bar{t}=K\right)$.

This result was reached by Nernst from the consideration of the case of a single membrane interposed in the path of a current. It is evident that such a system offers conditions much simpler than those of an irritable tissue, which typically consists of a bundle of cells or fibrils. Hill ${ }^{x}$ and Keith Lucas ${ }^{2}$ have pointed out that in considering the case of the living cell or nerve-fiber. with its small linear dimensions, it is necessary to tahe

I Journal of Physiology, XL (1910) 190.

${ }^{2} I b i d$., p. 225 . 
into account the processes at the two opposite surfaces of each element, since if these surfaces are close enough together, there is mutual interference of the two processes, and more complex conditions have to be assumed. Yet the fundamental condition assumed by Nernst's theory -a membrane partitioning an electrolyte solutionexists in the living tissue, hence polarization effects must result when a current is passed; and it has been found that the foregoing law relating the current-intensity to the duration required for a constant polarizing effect holds true also for the stimulating effect of the current within a considerable range of intensities and durations, and especially for higher intensities. This general result, that polarizing effect and stimulation run closely parallel, indicates that stimulation is a consequence of the polarizing action of the current.

Hermann $^{x}$ and others had previously referred the stimulating action of the current to its polarizing action, since in any tissue the presence of a reverse current (polarization current) can always be demonstrated immediately after the passage of a brief constant current. A high degree of polarizability is characteristic of living tissues, and this peculiarity is undoubtedly dependent on the semi-permeable properties of the cell membranes, since the polarization current is greatly diminished at death, at which time the membranes lose semipermeability, as already pointed out. Both polarizability and semi-permeability are thus manifestations of the same condition, hindrance to diffusion of ions. Lapicque has shown that the polarization currents obtained from dead partitions (parchment or bladder

${ }^{x}$ L. Hermann, Handbuch der Physiologie, Leipzig, II (I879), Part I, 193. 
membranes) are related to the intensity and duration of the polarizing current in the same manner as the stimulating effect of a current traversing a living irritable tissue; i.e., to produce a constant polarization current, the product of the intensity of the polarizing current into the root of its duration must be constant. ${ }^{8}$ It thus appears certain that the primary or initiatory process in electrical stimulation is the production of a certain critical degree of polarization at the semi-permeable membranes of the irritable tissue. In other words, the current stimulates by means of its polarizing action, i.e., by producing a potential difference (or by altering an already existing potential difference) between the external and the internal faces of the semi-permeable plasma membranes.

It should be noted that in itself this result throws little light upon the special physiological nature of the stimulation-process; it merely defines the physical conditions under which this process is initiated. The process itself, as just pointed out, has its specific peculiarities which are independent of the nature of the exciting agent. It is, however, an important theoretical advance to recognize that polarization changes are involved in all forms of stimulation. That this is the case is further shown by the invariable participation of bioelectric currents in stimulation processes; these currents, like any others traversing the tissue, must cause changes of polarization at the cell surfaces. According to the present view, the spread of excitation is due to the secondary stimulation-effects resulting from the polarizing action of such currents.

${ }^{x}$ Lapicque, Compt. rend. soc. biol., LXIII (1907), 37. 
We are thus led to consider the kinds of effect which changes in the electrical polarization across the cell surface may have upon chemical processes occurring in this region.

It should first be noted that various phenomena occurring at metallic surfaces and involving electrolysis have been shown to follow the same "square root law" as the stimulation process. Bredig and Kerb found this to be true for the influence of alternating currents in initiating the characteristic rhythmical action in the mercury hydrogen peroxide system, which, as we have seen, resembles closely the passive iron model in its mode of activity; the same was found by Wilke and Meyerhof in the electrolytic oxidation and reduction of chromic salts and chromates at platinum electrodes. ${ }^{x}$

Whenever a sufficient uncompensated potential difference is established between an electrode and a solution, as in any battery with closed circuit, the conditions for chemical change are present; there is a transfer of electricity associated with a chemical decomposition or other reaction (oxidation, synthesis, etc.) at the interface. It is well known that a certain critical decomposition-voltage must be exceeded in order to carry out any definite electrolysis, e.g., of a metallic salt; and if the cell surface possesses the general properties of an electrode, the chemical reactions there occurring must be subject to similar conditions. The need for a certain minimal or "threshold" current-intensity in stimulation is thus explained; it is evident that if the foregoing theory of transmission is well founded the potential

${ }^{x}$ Bredig and Kerb, loc. cit.; Wilke and Meyerhof, Arch. ges. Physiol., CXXXVII (I9I0), I. 
difference of the local bioelectric circuit in a conducting tissue like a nerve must exceed the critical value required for the electrochemical process which initiates the chemical reaction of stimulation. 'The general nature of the conditions will be considered more fully later when the phenomena of transmission are discussed in detail. For the present we may conclude that the significance of the polarization change involved in electrical stimulation is simply to furnish the condition required for some critical chemical decomposition at the cell surface. Presumably this chemical change alters locally the physical properties of the surface-film in such a way as to involve local breakdown or increase of permeability; and then, just as in the passive iron model, an automatically self-propagating wave of chemical decomposition is initiated. In this process the altered and the unaltered portions of the cell surface act as two electrode areas, in a manner analogous to that observed in the passive wire and similar systems during transmission.

In living tissues the conditions are more complex than in the simple model considered by Nernst, which takes account of only one of the conditions of electrical stimulation. Two chief conditions which this simple theory disregards are: (I) the existence of a critical threshold current-intensity, independent of duration; and (2) the character of the response to currents of changing intensity. Nernst's theory, however, explains the essential fact of polar stimulation, in addition to assigning a definite condition, viz., change of polarization, for the initiation of the stimulation-process. On the basis of the law of polar stimulation we may now say further that a change of polarization in a definite dircclion, such as 
to render the external layer of the solution in contact with the cell surface less positive than before, i.e., a depolarization, is the critical or initiatory event in stimulation. ${ }^{\mathbf{x}}$

But a current of too weak intensity, or one rising to its maximum too slowly, will not stimulate, whatever its duration. These discrepancies from the simple polarization theory must be referred to the special properties which the irritable tissue possesses by virtue of being a living structure. Apparently the irritable element is able to compensate slight or gradual changes of polarization as a part of its general regulatory capacity. Thus, if a current be led gradually into a nerve or muscle, a considerable intensity may be reached without stimulation. But if then the current be suddenly broken, stimulation results. This behavior seems to imply that while the current is gradually increasing, the cell by some regulatory process maintains its normal or resting physiological polarization essentially unaltered. During the flow of the external current, part of the polarization at the cell surface must depend on the presence of this current, which steadily conveys ions to (or from) the surface. When this influence is suddenly withdrawn, by breaking the current, the effect is to alter the polarization more rapidly than can be compensated by the activity of the cell, and stimulation results. The fact that the rate of change to which the irritable element can thus adjust itself without undergoing stimulation is rapid for rapidly reacting tissues (i.e., those with brief chronaxie) and gradual for "slow" tissues indicates that

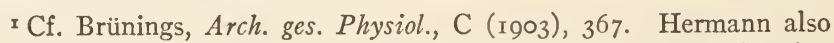
recognized that the polarization change of stimulation is in the direction of rendering the external surface of the irritable element less positive than before (loc. cit.). 
some specific chemical process, whose rate is determined by the characteristic metabolic properties of the tissue, is what preserves the normal resting state of the irritable elements. Thus we may imagine the material of the surface-film as being constructed and replaced as rapidly as it is removed by the chemical (reducing) action of the current; under these conditions the film (with its resting polarization) remains unaltered and no stimulation results. But if the rate of removal exceeds the rate of replacement, the consequence is alteration of the film and stimulation. Conditions of essentially this kind exist in the passive iron model, which shows a similar type of behavior.

\section{STIMULATION BY CONSTANT CURRENTS}

A typical case of stimulation by the constant current will illustrate how the stimulating effect varies with the duration of the stimulus. The following table from

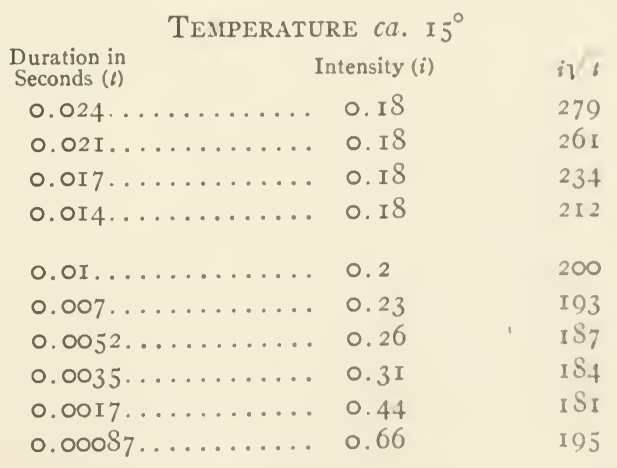

Keith Lucas $^{x}$ gives results obtained with the sartorius muscle of the frog. Constant currents of known intensity

IJ Physiol., XxXVII (1908), 475. 
were passed through the tissue by non-polarizable electrodes, and the minimal duration required for stimulation was determined by varying the distance between two contacts (one making, the other breaking the current) in a swinging pendulum.

The essential feature of these results is that below a certain definite intensity of current (o.I8 units), increasing the duration has no effect in lessening the intensity required to stimulate; i.e., weaker currents will not stimulate, whatever their duration. This intensity represents the critical or threshold value for any current. But with stronger currents, the duration required for stimulation becomes less as the intensity increases, and a close approximation to Nernst's square root law is found. This signifies that a certain minimal change of polarization is required to initiate the stimulation process; with currents above a certain critical intensity this polarization is attained with briefer and briefer durations as the intensity is progressively increased. Lapicque and other observers have obtained similar results. The current of threshold value, i.e., of the least intensity that will stimulate with any duration, must traverse the tissue for a certain minimal time in order to stimulate. This time is characteristic for the tissue in question, and apparently is a direct function of the rate of certain specific metabolic processes; probably those concerned in the alteration of the surface-film, as indicated by the duration and other features of the refractory period (see below). According to Keith Lucas and Mines, the length of this minimal time varies with the temperature of the tissue in accordance with a somewhat low temperature-coefficient, similar to that of diffusion 
$\left(\mathrm{Q}_{\mathrm{I} O}=\mathrm{I} \cdot 3\right) .^{\mathrm{x}}$ For the irritable tissues of the frog. Lucas ${ }^{2}$ gives the following determinations (at $\mathrm{Cl}$. I $3^{\circ}$ ): substance $\beta$ (nerve end-plate) of the sartorius, .oor second; motor nerve-trunk, .003 second; muscle fiber (sartorius), .02 second; ventricle, 2 seconds; for smooth muscle the period is much longer (several seconds). Lapicque finds the least effective duration of the minimal stimulating current to vary widely for the muscles of different animals, and gives the following data: ${ }^{3}$

\section{Muscle}

Gastrocnemius (rana esculenta)

Gastrocnemius (r. temporaria)

Rectus abdominis (r. esculenta)............ .

Gastrocnemius (bufo vulgaris)........... . I.3 sec.

Foot of snail (helix pomatia) .............. $045 \mathrm{sec}$.

Foot of snail (solen marginatus) . . . . . . . . . $075 \mathrm{sec}$.

Ventricle of tortoise (testudo græca) .......... $\$_{2} \mathrm{sec}$.

Claw muscle of crab (carcinus mœnas)...... $30 \mathrm{sec}$

Mantle muscle of mollusc (aplysia punctata).. .So sec.

These determinations illustrate the specificity of this time-factor for different animals. It is interesting to note that the relocities of the motor nerve impulses in different animals vary in a closely parallel manner. To designate this characteristic time-factor in the electrical stimulation of different irritable system: Lapicque has introduced the term "chronaxic." As now defined, the term has reference to the least duration required by a current of exactly twice the threshold intensity (or so-called "rheobase").

${ }^{x}$ Lucas and Mines, Journal of Physiology, XXXVI (1007), 334. Lucas, ibid, XXXIX (Igro), 46 r; cf. p. 472.

${ }^{2}$ Lucas, Journal of Physiology, XL (1910), 225; of P. 245 .

${ }^{3}$ Lapicque, Compt. rent. soc. biol., LVII (1905), 503. 
The characteristic time-factor or chronaxie of a tissue also expresses itself in the rate of variation of intensity required by the stimulating current; this rate is greater the briefer the chronaxie; it is also greater the more rapidly the stimulation-process develops in the tissue, as indicated by the rate at which the accompanying bioelectric variation rises to its maximum. The chronaxie also varies directly with the duration of the summation-interval for subminimal stimuli. ${ }^{x}$

\section{STIMULATION BY CURRENTS OF CHANGING}

INTENSITY

The stimulating effect of a current of continuously changing intensity, or of a change in the intensity of a current already traversing the irritable tissue, varies, in a manner which is characteristic for the tissue, with the rate of change, and is largely independent of the actual intensity. It is significant that this rule, relating stimulating effect to rate of change, applies also to mechanical, chemical, and other forms of stimulation, in all of which a sudden change is more effective than a gradual one. A general property of living matter is apparently here involved. In the activation of the foregoing metallic model (passive iron wire in nitric acid) the same rule holds; e.g., in order to activate the metal mechanically by scraping with glass, the movement must be rapid; a slow movement is ineffective. Similarly in electric activation a current which is gradually increased up to a sufficient intensity has no effect, while one of the same intensity, attained suddenly, causes instant activation.

${ }^{x}$ Cf. Lucas, Journal of Physiology, XXXIX (I9I0), 463; cf. p. 470. 
The rate of change which a current requires in order to stimulate a tissue varies with the nature of the tissue, and is a function of the characteristic chronaxie. When the chronaxie is brief, the rate of change must be rapid. When the rate of change of an increasing current is gradual, a greater final intensity of current is needed for stimulation than when this rate is rapicl. The following observations of Lucas, on the stimulation of the frog's sartorius, illustrate the conditions for a single typical tissue. The rate of change of the exciting current was controlled by varying the rate of movement of a shutter which opened and closed a slot in a partition set across a zinc sulphate solution, forming part of the stimulating circuit. ${ }^{x}$ Comparison was made between the current-strength required: (I) when the circuit was closed instantaneously; and (2) when the intensity was increased from subminimal to a stimulating value at varying rates. The muscle was also stimulated by currents of the same linear gradient or rate of change under two conditions, (A) while immersed in pure 0.7 per cent $\mathrm{NaCl}$ solution and (B) in a mixture of 0.65 per cent $\mathrm{NaCl}$ plus 0.05 per cent $\mathrm{CaCl}_{2}$. The following results are typical:2

Time Required to Reach Full Intensity (Seconds)

o (instantaneous)...

o. I sec...........

$0.27 \ldots \ldots \ldots \ldots$

$0.50 \ldots \ldots \ldots \ldots$

$0.97 \ldots \ldots \ldots \ldots$

\section{Strength of Current Required for Stimulation}

A $(\mathrm{NaCl})$

$\mathrm{B}\left(\mathrm{NaCl}+\mathrm{CaCl}_{6}\right)$

$\begin{array}{ll}\text { I } & \text { I } \\ \text { I } & \text { I } \\ \text { I. O5 } & \text { I.OF } \\ \text { I. I } & \text { I. } 27 \\ \text { I. I } & \text { I. } 5\end{array}$

${ }^{1}$ Lucas, Journal of Physiology, XXXVI (1907), 253.

${ }^{2}$ Lucas, ibid., XXXVII (1908), 459; cf. p. 473. 
The more slowly the current changes its intensity the less effective it is as a stimulus. The sensitivity to rate of change varies with temperature and the composition of the medium; the necessary rate of change is greater at higher temperatures; it is also greater when calcium is present (B) than in the pure $\mathrm{NaCl}$ solution (A). According to Lucas, "an increase in the concentration of the calcium would appear to necessitate a more rapid concentration of the ions concerned in excitation."I Observations by Mines, on the minimal duration of the threshold current of constant intensity, ${ }^{2}$ have shown that in this case also the duration is briefer when $\mathrm{Ca}$ is present. Such facts indicate that the chronaxie of a tissue is determined not only by its specific constitution but also by the external conditions to which it is exposed. This is well shown in certain studies, by Adrian, on the effects of peripheral nerve injury. ${ }^{3}$

The chronaxie of a tissue appears to be closely related both to the rate of response and to the rate of recovery of the irritable elements. Thus it shows a close correlation with the characteristic duration of both the bioelectric variation of the tissue and the refractory period. The more slowly a tissue responds to a constant current, i.e., the longer the minimal duration of the current of threshold intensity, the more gradual is the rate of change required for excitation by a current of changing intensity. The length of the summation-

IOp. cit. (1908), p. 480.

2 Cited in Lucas' paper, op. cit. (I908), p. 472.

${ }_{3}$ The chronaxie of a muscle with nerve supply interrupted increases progressively until innervation is re-established (Archives of Radiology and Electrotherapy, May, I917). 
interval also appears to be determined by the same conditions.

\section{SUMMATION}

The phenomenon of summation is of great importance in the analysis of the stimulation process. It shows clearly that a single subminimal stimulus produces an effect on the tissue, but that this effect is transient; within a certain brief time the tissue resumes the same condition as before the stimulus. But if before this time has elapsed a second similar stimulus is applied, its effect is added to that of the first, and the critical level of disturbance required to initiate an excitationwave may be reached. The second stimulus, in order to be effective, must be sent in before the effect of the first has subsided; and the more rapid the rate of this subsidence the shorter is the summation-interval. The summation-interval is therefore defined as the longest interval separating the successive subminimal stimuli of an effective series of two or more such stimuli. ${ }^{\mathrm{s}}$

This interval is shorter than the least duration of the exciting current of threshold intensity; and its precise duration varies with the intensity of the subminimal stimuli employed. Lucas gives the following intervals for different frog's tissues at $13^{\circ}$, using two subminimal electric stimuli (induction shocks) which were 5 per cent below the strength required for stimulation by single stimuli. $^{2}$

Motor nerve (sciatic) .....000 $-.0005 \mathrm{sec}$.

Muscle (sartorius).......001 1-.0019 sec.

Ventricle............ .0 $8 \mathrm{sec}$.

${ }^{x}$ Cf. Lucas, Journa! of Physiology, XXXIX (1910), 412.

'Op. cit. (1910), pp. $466 \mathrm{ff}$. 
When the shocks were ro per cent below the threshold, the interval was much shorter.

The summation-interval is thus longer the more gradual the excitation-process (the longer the chronaxie) of the tissue. It varies with temperature and with the state of the tissue. Lucas finds the temperaturecoefficient to be low $\left(\mathrm{Q}_{\mathrm{IO}}=c a\right.$. I.3), a fact suggesting that purely physical changes, e.g., diffusion-processes (which have a similar temperature-coefficient), are chiefly concerned in the return of the tissue to the normal after a slight disturbance. ${ }^{\mathrm{I}}$ The influence of the inorganic salts is again highly interesting. The presence of $\mathrm{Ca}$ shortens the summation-interval, just as it shortens the minimal duration of the threshold constant current and increases the rate of change required for stimulation by a changing current. ${ }^{2}$

According to Lucas and Mines, the effect of temperature on the minimal duration of the threshold current is the same as on the summation-interval. Such facts again emphasize the distinction between the local change produced by the stimulating agent and the propagated

I Cf. Lucas' discussion, op. cit., p. 473. It is noteworthy that the time required for the return to the normal properties after complete stimulation, as measured by the length of the refractory period, is much longer than the summation-interval, and that the temperature-coefficient of this return or recovery process is high $\left(\mathrm{Q}_{\mathrm{I}_{0}}=c a .3\right)$; these facts indicate that chemical processes play the chief part in the recovery from a complete stimulation. The "local change" may thus be of a purely physical kind (e.g., polarization change), while in the complete or propagated excitation the chemical factor is essential. This conclusion agrees with the fact that the refractory period is much longer than the summation interval. Apparently the former represents a period of metabolic and structural restitution.

${ }^{2}$ Lucas, op. cit., p. 472 . 
effect or stimulation-process proper. 'The former is, or may be, a purely physical change; in electrical stimulation its essential feature is apparently a change of polarization resulting from changes of ionic concentration at the cell surface. This change does not initiate a propagated effect unless it exceeds a certain critical limit, and unless the state of the tissue is favorable: thus in an anæsthetized tissue the local change of polarization is produced by a current, but no propagated excitation follows. The differences between the physical conditions and manifestations of the two processes, local change and propagated disturbance, and the differences in their temperature-coefficients show that the propagated process is more complex than the initiatory local process and includes chemical or metabolic factors among its chief components.

\section{GENERAL NATURE OF STIMULATION CIIANES}

The factors determining the characteristic chronaxic of a tissue would thus appear to be largely factors determining the rate at which the critical polarization change occurs in the irritable elements. This rate depends on the rate of movement of ions and also on the special structural conditions within the tissue. An important advance in the theory of the local change has been made by Hill, ${ }^{x}$ who has modified Nernst's simple theory and brought it into closer conformity both with the facts of organic structure and with the actual behavior of the tissue in electrical stimulation. Hill points out that in any case of electrical stimulation the concentration-changes at he semi-permeable surfaces

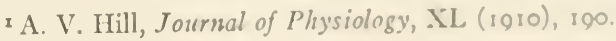


situated a short distance apart must be considered; these are the surfaces where the current-lines intersect the two opposite faces of the irritable element. If these surfaces are close together, the diffusion-gradient set up by a given current is steep, and hence the backdiffusion opposing the polarization is relatively rapid. The production of the critical polarization change will then require a stronger current than with the membranes far apart. This conception explains also why a current passing crosswise through a nerve or parallel-fibered muscle is so much less effective than one passing lengthwise. Hill's calculation leads him to the formula, $i=\frac{\lambda}{\mathrm{I}-\mu \theta^{t}}$, for the conditions of stimulation by a constant current, where $\lambda$ is a direct function both of the proximity of the two membrane-surfaces concerned (proximity being the reciprocal of the distance apart) and of the rate of movement of the ions; $i$ represents the intensity of the current, $t$ its duration, and $\mu$ and $\theta$ are constants having reference to the conditions of movement of the ions in the tissue. This formula gives a remarkably close agreement with observation through a wide range of intensities. A further essential feature of Hill's theory is its recognition that the polarization change is in reality merely the determining condition of a chemical change which must proceed at a certain minimal rate in order to cause excitation. From this point of view it is possible to understand why not only the degree of polarization attained, but also the rate at which this critical degree is reached determines whether stimulation shall be initiated or not. The stimulation process is a process sui generis, distinct from the initiatory physical change; 
yet it is of such a nature as to be initiated only by physical changes proceeding at more than a certain rate.

We are thus led again to consider those special properties of the living system which it possesses by virtue of being living; i.e., metabolically and synthetically active. The features above of stimulation cannot be understood except by reference to what is distinctive in vital processes as such. Yet it is to be noted that the case of the living system is by no means unexampled in the respect just considered. There are many natural processes in which, if a certain effect is to be produced, the effecting agent must act at more than a certain minimal rate; if it acts slowly, the effect fails entirely. For example, a swift current of air will extinguish a candle flame, while a slow one will not; a rapid stroke will ignite a match, a rapid projectile penetrates a plate, a rapid attack succeeds in war or on the football field. The lighting of a match shows many analogies with stimulation. Ignition occurs at a certain critical temperature, which is attained when the match head is drawn uniformly over a rough surface for a certain time at more than a certain rate. If the movement is too slow, ignition will never occur; in this case the stationary condition at which the gain of heat from friction is equal to that lost to the surroundings by conduction and radiation is reached at a temperature below that of ignition. A movement at a certain rate must last for a certain time, which is shorter the more rapid the rate. Summation phenomena and summation intervals may also readily be demonstrated in this system; i.e., a succession of brief strokes but not a 
single stroke will ignite if the interval between the strokes is not too long.

It will be evident that the general condition common to all cases of this kind is that two active processes or sets of processes, with resultants acting in opposite directions, are concerned; the equilibria are "dynamic" rather than static. In an irritable tissue through which a uniform electric current is flowing, the effect which the current produces in the direction of stimulation is presumably counterbalanced by contrary processes depending largely on the metabolic or synthetic activity of the tissue. Interruption of an already existing uniform current, as well as its sudden increase or decrease, disturbs this equilibrium and may result in stimulation.

Further analysis of the process of stimulation requires, therefore, a consideration of the special nature of the processes occurring in the irritable protoplasmic system.

According to the foregoing conception of the conditions of stimulation in living cells, the primary or initiatory process is a local alteration of the protoplasmic surface-film, or "plasma membrane," of the irritable element. This alteration has a self-propagating character, like that shown by other chemically alterable surface-films at the boundary between two electrically conducting phases, and leads secondarily to the characteristic manifestation of cell-activity, or response. If this conception of the stimulation process is a true one, all forms of stimulation should exhibit definite evidence of accompanying surface-processes of the kind indicated.

Certain effects which apparently accompany all forms of stimulation and activation, whatever the special nature of the response may be, constitute evidence of 
this kind. These are: (I) The bioelectric variations: (2) the presence of a refractory or temporarily inexcitable period immediately following stimulation; and (3) a temporary loss of semi-permeability or increase in the permeability of the cell surface to water-soluble sub, stances.

We have seen above that the electric current is at universal stimulating agent; and that, conversely; when irritable tissues respond, they give rise to electric currents which traverse the surroundings and may be there detected by appropriate means. Similarly, mechanical or chemical alteration of the cell surface causes excitation and also gives rise to bioelectric currents. Further, during many forms of normal excitation there is direct evidence that the surface layer of protoplasm undergoes a sudden and pronounced change in its properties, one effect of which is to increase the permeability to water-soluble substances; in many cases this change is distinct and easily demonstrated; e.g., the turgor mechanisms of plants, gland cells, and egg cells during activation; in others (nerve, muscle) the indications of changing permeability are indirect. The loss of irritability during the refractory period is also in harmony with the hypothesis that the surface layer breaks down or is otherwise altered during stimulation, since semi-permeability. implying electrical polarizability, is apparenty essential for stimulation; any temporary loss of semi-permeability must therefore involve loss of irritability.

The strongest evidence that the surface-change is the essential and primary change in stimulation is the constancy with which the foregoing three manifestations of stimulation are associated. Especially significant also 
is the fact that closely analogous phenomena are observed in the inorganic models considered above (mercury hydrogen peroxide catalysis, passive iron), in which the process of activation is known to depend upon the electrolytic disintegration of thin interfacial films. In the temporary activation of passive iron, the dissolution of the oxide-film involves (I) a change of potential, (2) a marked increase in permeability, allowing ready access of the acid to the metal, and (3) a delay in the recovery of the former state of susceptibility after the return of passivity. It seems highly improbable that those parallels are accidental; the indications are that they are expressions of an underlying identity in the essential structural constitution and conditions of activity of the living system and of the inorganic model. The essential features of structure and composition common to both systems are, briefly; (I) the presence in both cases of a thin film separating two electrically conducting phases, one or both of which is an electrolyte solution, and (2) the susceptibility of the film to alteration under the influence of electric currents (breakdown or construction by local electrolysis). Before dealing in greater detail with these parallels and their bearing on the problem of the essential constitution of living matter, it will be necessary to review briefly the essential facts which have been established with regard to the foregoing three general accompaniments of stimulation. 


\section{CHAPTER XIII \\ BIOELECTRIC PHENOMEN.I}

A complete review of this large field of research is not possible in the space at our disposal. ${ }^{x}$ It is necessary, however, to consider in some detail the chief facts bearing on the present problem; these may be conveniently grouped under the two headings: (I) bioelectric potentials in resting cells and tissues; and (2) variations of bioelectric potentials in relation to physiological activity.

\section{RESTING BIOELECTRIC POTENTIALS}

The existence of potential-differences between the resting cell or other protoplasmic element and its surroundings has in itself nothing unexampled or surprising. Typically such potentials are found at all phaseboundaries unless special compensating conditions are present. The conditions on either side of the interface are asymmetric with respect to chemical composition and physical condition, and corresponding to this assymmetry there is an electrical asymmetry or potentialdifference. The facts of electrical convection and electrical endosmose show the presence of potentialdifferences between all kinds of insoluble mąterials and the adjacent layer of solution. These potentials

' For an exhaustive account of the earlier work, cf. Biedermann's Electrophysiologie, English translation. For a more recent account, cf. Bernstein's Electrobiologie (1912), and the article of Garten, "Produktion von Elektrizität," in Winterstein's Handbucls der scrgl. Physibl, III (I910), I05; also the interesting but more special work of Bose, Comparative Electrophysiology (1907). 
are known to vary with the composition of the nonaqueous phase and with the electrolyte content of the aqueous phase; i.e., ions have a special influence, although surface-active substances other than electrolytes may also have an effect, as already shown. The case of the living cell falls partly in this general category. Suspended cells travel in the electric field, usually toward the anode; and the rate and even the direction of this travel may be changed, just as in non-living suspended particles, by changing the electrolyte content of the medium; especially active in this regard are $\mathrm{H}$ ions and the ions of polyvalent metals. Constant currents passed through living tissues (muscle) effect transport of fluid. This is a special case of electrical endosmose; evidently any passage of electricity through a cell must involve some displacement or transport of fluid, a fact which must be considered in relation to physiological processes like secretion, absorption, and cell-division. The potentials between suspended particles and suspension-media are apparently in large part adsorption potentials; their range is comparatively narrow, usually between 0.02 and 0.05 volt; according to Freundlich they represent the potentials between an adhering immobile layer of solution and the mobile layer adjoining. ${ }^{x}$

These facts, while relevant to the general theory of the bioelectric potentials, do not in themselves explain sufficiently the special peculiarities of the latter. Apparently the closest resemblances are with electrode potentials; e.g., those between a metal and an adjoining solution. From the physiological point of view the most

${ }^{x}$ Freundlich, Kapillarchemie, p. 243; Report on the Physics and Chemistry of Colloids, Faraday Society and Physical Society of London (London, I921), p. I46. 
significant fact is that the demarcation potentials (shown in the injury-currents of muscle and similar phenomena) vary with the condition of the protoplasmic surface layer. The loss of semi-permeability accompanying death is always associated with a decline or disappearance of the demarcation potential, a fact indicating that the latter depends on the presence of a semipermeable partition between the internal protoplasm and the surrounding medium. Such a partition allows the existence of permanent differences of electrolytecontent between the solutions adjoining the outer and inner faces of the plasma membrane, and so provides the asymmetric conditions necessary for a potentialdifference. Just why this potential-difference should have the observed orientation (positive externally) and range (of the order of 0.05 to $0 . \mathrm{I}$ volt) is not entirely clear; possibly these conditions are referable to a higher total electrolyte content of the cell interior as compared with the surroundings, or to a preponderance of certain ions (e.g., $\mathrm{H}$ ions) in the cell interior. The chemical relations between the ions present in solution and the materials composing the surface-film are undoubtedly an important factor, and it seems probable that oxidationreduction potentials and adsorption potentials (in the Freundlich sense) are also concerned. The total potential as observed thus represents an additive effect.

It is evident, however, that the demarcation potential depends primarily on the special physical and chemical properties of the cell surface, since whatever modifies the structure or chemical character of the protoplasmic surface layer also alters the potential. This is shown by the conditions under which the so-called currents of 
injury arise. Mechanical or other injury and the application of cytolytic substances, which demonstrably increase permeability, always lower the potential; i.e., the altered region becomes negative relatively to unaltered regions. This effect is often reversible if the tissue is not exposed too long; thus potassium salts render a voluntary muscle locally negative, in accordance with their permeability-increasing action; and if the tissue is soon afterward bathed in Ringer's solution, the original isoelectric condition returns. Any local alteration which impairs semi-permeability thus induces local negativity; i.e., decreases the potential-difference between the protoplasm and the surroundings. The fact that the variation of potential is always in a negative direction is consistent with the theory that the normal negative variation accompanying stimulation is also the effect of an alteration of the cell surface, involving a temporary and rapidly reversed increase of permeability. We may thus understand why the bioelectric variation of stimulation is similar in its direction and range to that accompanying loss of semi-permeability, while differing in being reversible or evanescent.

It has long been recognized that variations in the permeability of a semi-permeable partition separating two electrolyte solutions must involve variations in the potential difference across the partition, and the chief modern attempts to explain the bioelectric potentials have been based on this ground " membrane theory" of Ostwald, I $890,{ }^{\mathrm{I}}$ followed by Cybulsky, Bernstein, ${ }^{2}$

I Ostwald, Z. physik. Chem., VI (I89o), 7I.

${ }^{2}$ Cybulsky, Bull. Acad. Sci. de Cracovie (I898), p. 231; Bernstein, Arch. ges. Physiol., XCII (r902), 52r. For other references cf. Höber's textbook, op. cit., p. 579. 
and others). Ostwald's original suggestion was that the plasma membrane may act as an "ion sieve," allowing the cations of some intracellular electrolyte to pass but not the anions. This hypothesis was adopted by Bernstein as affording a point of view from which the sudden fall of potential during stimulation might be explained; at this time Bernstein supposed the membrane to become permeable to both classes of ions. The actual conditions, however, are undoubtedly more complex, and include other factors than the simple diffusion potentials considered by Ostwald and Bernstein. Nevertheless, it must be recognized that the breakdown of a semi-permeable partition between the protoplasm and its medium (the two adjoining electrolyte solutions concerned) must decrease the potential between the two, whatever the detailed conditions of this potential may be. The "membrane theory" of the bioelectric potentials need not necessarily have the form of a modified diffusion theory, as some of its opponents seem to have supposed. More recent developments of this theory have aimed at correlating the bioelectric phenomena with the chemical as well as the physical processes occurring in the protoplasmic boundary layers. It may now be taken as well established that the cell surface possesses electrode-like properties, and that in the determination of its electromotor behavior other conditions enter than merely a selective or differential hindrance to the diffusion of ions.

The work of Macdonald ${ }^{\mathrm{I}}$ is of special interest since it first showed that the demarcation-potential of nerve varies with the concentration of the salts in the adjoining

'Macdonald, Proceedings of the Royal Socicly, LXVII (1900), 310. 
solution in the same manner as the potential difference between a metallic electrode and its adjoining solution (e.g., $\mathrm{Zn}$ in $\mathrm{ZnSO}_{4}$ solution). When the demarcation potential between the cut surface of the sciatic nerve and an uninjured area one centimeter distant was measured (by the usual compensation arrangement), after leaving the nerve for five minutes in differently concentrated solutions of a given salt, results of the following kind were obtained. (The potential in the original physiological salt solution is represented in the bracketed expression by $E$.)

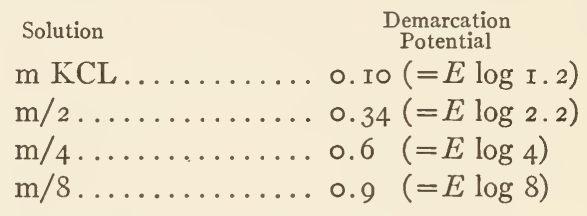

It will be noted that the potential difference increases with increasing dilution, and very nearly in direct proportion to the logarithm of the dilution. This is the characteristic relation found in electrode potentials, and expressed by Nernst in the formula $E=R T \log \frac{c_{1}}{c_{2}}$ where $c_{I}$ represents the concentration corresponding to zero potential (equivalent to that required to compensate the ionic solution-pressure of the metal), and $c_{2}$ the concentration of the ions in solution; thus with zinc in contact with $\mathrm{ZnSO}_{4}$ solution, the potential decreases with increase in the concentration of the zinc ions in a logarithmic curve. The fact that a similar relation is found with the living tissue, and that the results obtained are reversible, as Macdonald found, shows that the living tissue behaves as if its surface were an electrode reversible 
with respect to the cations of the solution. Results similar to the foregoing were obtained also with $\mathrm{NaCl}$ and $\mathrm{HCl}$.

More recently Loeb and Beutner, ${ }^{\mathbf{X}}$ in an extended and important series of researches, have shown that the characteristic logarithmic relation between the concentration of the ions in the solution and the potential difference holds for organic membranes of a variety of kinds and also for solutions of lipoids in organic solvents. The organic membranes act as if they were reversible to cations as a class. This result is highly significant, for it seems to imply that reversible combinations between these ions and components of the membrane (e.g., proteins or lipoids) occur, and that the formation of these combinations is the essential factor determining the potential equilibria observed in a given solution. Just as a metallic electrode, like $\mathrm{Zn}$ in contact with a solution of $\mathrm{ZnSO}_{4}$, may be regarded as "dissociating off" zinc ions until an equilibrium (to which corresponds a definite potential difference) exists between the ions tending to pass into solution from the metal and those already in solution, so in the case of a salt solution in contact with an organic membrane a certain potential difference corresponds to the equilibrium existing between the ions in solution and the ion-membrane compounds formed by the combination of these ions and the membrane components (proteins, etc.). Any increase of the ions in solution decreases the potential difference in logarithmic ratio.

${ }^{3}$ Loeb and Beutner, Science, XXXIV (igri), 884; XXXVII (19r3), 672; Biochem. Zeitschrift, XLI (1912), r, and XLIV, 303; LI (1913), 288, 301; LIX (19r4), 195. Cf. also Beutner, ibid., XLVII (19ז2), 73. 
The demarcation potentials of living tissues appear to exemplify this general condition. ${ }^{\mathrm{I}}$ In the usual method of studying these potentials in muscle or nerve the normal cell surface in part of the tissue is first altered by mechanical or other means. The electrodes leading to the galvanometer then touch two surfaces, altered and normal, which differ in their physico-chemical condition; a corresponding potential difference is shown, the injured region being negative. Hence the term "alteration current" for the current between the two regions. Loeb and Beutner found the same to be true for a simple organic membrane like an apple skin; when one region is crushed, this region shows itself negative to an unaltered region; potential differences of 20 to Ioo millivolts were observed in different experiments, the values varying with the concentration of the salt solution in contact with the tissue. ${ }^{2}$

Loeb and Beutner found also that the potential changed with changes in the concentration of the surrounding solution in a manner similar to that observed by Macdonald for nerve. To produce a constant arithmetic change in the potential-difference, the concentration of the salt had to be changed in a constant ratio; i.e., to a geometric series of concentrations

${ }^{x}$ The importance of the Donnan membrane potential (potential across a membrane permeable to only a part of the ions present), in the case of protein solutions separated from electrolyte solutions by collodion or similar membranes, has recently been demonstrated by Loeb in an important series of researches (summarized in his book, Proteins and the Theory of Colloidal Behavior). In this case a colloidal ion (protein) is the one to which the membrane is impermeable. In living tissues, however, with protein ions in about equal concentration on both sides of the membrane (in protoplasm and in lymph) this source of potential can scarcely play a part.

${ }^{2}$ Biochem. Zeitschrift, XII (I9I2), r; cf. p. 22. 
corresponds a linear series of potentials, the relation characteristic of electrode-potentials in general. 'That the effect observed in any single case depends on the special character of the surface was shown in a series of experiments in which Loeb and Beutner compared the effects of varying the concentration of the solution in contact with (A) the uninjured surface of an apple, and (B) a surface from which the skin had been removed. ${ }^{\mathrm{I}}$ In all such experiments one electrode in contact with the apple remained unchanged; the other was connected with the solution which was varied; the latter was in contact with another portion of the surface at some distance from the first electrode. A quadrant electrometer was used.

Potential Difference Observed with Solution in Contact

\begin{tabular}{|c|c|c|}
\hline 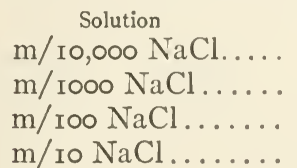 & $\begin{array}{l}\text { A (with uninjured skin) } \\
\quad+0.175 \\
\quad+0.146 \\
\quad+0.086 \\
\text { † } 0.023\end{array}$ & $\begin{array}{c}\text { B (with cut surface) } \\
\quad+0.056 \\
+0.036 \\
+\infty 0.0 \\
-0.022\end{array}$ \\
\hline
\end{tabular}

Both surfaces show the same kind of variation with varying concentration of electrolyte, but the altered area shows a smaller change of potential for a given change of concentration; thus on the average a tenfold dilution increases the positivity of the unaltered surface by about 0.06 volt, and of the altered by about 0.03 rolt. With every solution used the altered surface exhibits the lower potential; i.e., is negative relatively to the unaltered; the conditions also suggest that it represents an area which is reversible to anions as well as to cations, ${ }^{2}$

Loc. cit., I9I2.

${ }^{2}$ This would correspond to freer penetration by anions and an entrance of diffusion potentials in the total effect. 
while with the intact skin the reversibility relates to cations alone. In other words, the two regions exhibit different electromotor properties.

Further experiments ${ }^{\mathrm{x}}$ showed that the behavior of the organic membrane could be closely imitated by an arrangement in which a solution of a weak organic acid in a water-immiscible solvent is in contact with the salt solutions. In the arrangement already described:

calomel concentrated membrane- dilute calomel

$\begin{array}{ccccc}\text { electrode } & \text { salt } & \text { inclosed } & \text { salt } & \text { electrode } \\ \text { solution } & \begin{array}{c}\text { system } \\ \text { (e.g., apple) }\end{array} & \text { solution } & \end{array}$

the dilute solution is positive and becomes more positive with increasing dilution; such a chain is comparable to one containing a metal in contact with solutions of its salt; e.g.:

$\begin{array}{ccccc}\text { calomel } & \text { concentrated } & \text { metallic } & \text { dilute } & \text { calomel } \\ \text { electrode } & \mathrm{AgNO}_{3} & \mathrm{Ag} & \mathrm{AgNO}_{3} & \text { electrode }\end{array}$

in which also the side containing the dilute solution is positive; $\mathrm{AgCl}$ may be substituted for metallic silver with the same result. Similarly the arrangement:

$\begin{array}{ccccc}\text { calomel } & \text { concentrated } & \text { solution of } & \text { dilute } & \text { calomel } \\ \text { electrode } & \text { salt } & \text { salicylic } & \text { salt } & \text { electrode } \\ & \text { solution } & \text { acid in } & \text { solution } & \\ & & \text { salicylic } & & \\ & & \text { aldehyde } & \end{array}$

gave potential differences of a similar order to those found with the organic structure, and varying similarly with the concentration of the dilute salt solution. In other words, the surface of the non-aqueous phase acts

${ }^{-}$Cf. Beutner, Trans. Amer. Electrochem. Soc., XXI (IgI2), 219; XXIII (19I3), 40I; American Journal of Physiology, XXXI (1913), 343. 
in the same manner as an electrode reversible to cations. The remarkable feature is that the reversibility relates to salts of cations in general, and not only to those of a single cation, as in the case of silver or other metallic electrode. All of the alkali and alkali-earth cations (those of the chief physiological interest) gave typical results; thus with $\mathrm{KCl}$ the following observations were made; ${ }^{\mathrm{I}}$ the non-aqueous phase was salicylic aldehyde saturated with salicylic acid:

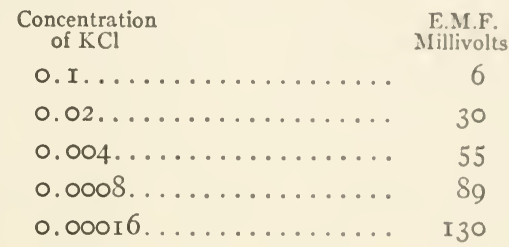

It was further shown that this effect is dependent on the acid character of the non-aqueous phase; i.e., on its ability to take up cations (reversibly) by salt formation; other solutions of weak acids, e.g., of benzoic acid in phenol, behaved similarly. But when the nonaqueous phase is basic in chemical character, the potential changes in the opposite direction, the dilute solution becoming more negative, instead of more positive, with increasing dilution, and the behavior is such as to indicate reversibility to anions. Beutner found this to be the case when the weakly basic compounds, aniline and toluidine, were used as the water-insoluble phase in an arrangement similar to the foregoing. ${ }^{2}$

${ }^{x}$ Cf. American Journal of Physiology, XXXI (1913), 347.

${ }^{2}$ For a complete account of Beutner's investigations cf. his recent book, Entstehung elektrischer Ströme in lebenden Geweben (Stuttgart, 1920). 
Such facts suggest that substances having the properties of weak acids determine the type of electromotor behavior shown by the cell surfaces in the demarcation potentials. Both proteins and lipoids (e.g.,lecithin) belong in this class. Loeb and Beutner ${ }^{\mathrm{I}}$ therefore carried out further experiments of the kind described, using solutions of lecithin in organic solvents, and found again the same relation between the concentration of the salt and the observed potential difference. When ro per cent solutions of lecithin in guaiacol were used, the behavior, both qualitative and quantitative, was found closely similar to that of plant tissues. Oleic and palmitic acids gave similar results, but not cholesterol. Extracts of various plant and animal tissues (muscle, brain, frogskin) in organic solvents also exhibited this behavior; a further interesting fact was that potassium salts had a greater influence than sodium salts in altering the potentials, a peculiarity which the authors ascribe to a greater solubility of potassium salts in the lipoid phase.

It would seem, therefore, as if the ordinary potential differences observed between altered and intact portions of the cell surface were phenomena of the same general type as those just described; i.e., referable to the presence of a water-insoluble phase containing weakly acid substances and forming a thin film or partition between two dissimilar electrolyte solutions, represented respectively by the living protoplasm and its surrounding medium. $^{2}$

I Biochem. Zeitschrift, LI (I9I3), 288.

${ }^{2}$ As to the specific nature of the electrolytes concerned, little definite can be said at present. In general the two cations whose concentration is higher inside than outside the cell are $\mathrm{K}$ and $\mathrm{H}$. A recent calculation 


\section{VARIATIONS OF BIOELECTRIC POTENTIALS}

The sudden fluctuations of potential accompanying normal vital processes like stimulation evidently require a different type of explanation. In such cases rapid and reversible alterations of the electromotor properties of the protoplasmic surfaces are apparently involved; these effects can only be referred to the chemical or metabolic processes characteristic of living matter. The external surface layer of the living cell consists of a thin film of chemically alterable material in immediate contact with the surrounding medium on the one side, and with the internal protoplasm on the other; it is, therefore, subject not only to purely physical changes, such as local thinning or interruption, but also to changes of chemical composition, resulting from variations in oxidative or other metabolism. Along with such alterations must go alterations in physical properties, thickness, permeability to electrolytes, acid or basic character, etc.; and these must alter correspondingly the electromotor properties of the cell surface. It has already been pointed out that the reversible variations of potential seen in the action-currents of tissues like muscle and nerve have a range closely similar to that of the demarcation-currents (ca. 0.05 volt); and this fact receives a consistent

by Adams favors the idea that the difference between the internal and external H-ion concentrations is an important factor in the bioelectric potentials (Journal of Physical Chemistry, XXVI [1922], 639).

Recently Rohonyi has opposed Beutner's conception of the importance of an oil-like phase in the determination of the bioclectric potentials; cf. his critique: Biochem. Zeilschrifl, CXXX (1922), 6S. He regards semi-permeability (permeability to water, but not to electrolytes) as the essential property. 
explanation on the hypothesis that dissolution ${ }^{\mathrm{I}}$ and re-formation of the semi-permeable surface layers of the cells are the main factors in the production of the normal electromotor variations. The specifically vital functions of metabolic construction and destruction which determine the physical properties of the cell structures would thus determine also the normal variations of the bioelectric potentials. The general physico-chemical conditions of these potentials are of a kind present at all phase-boundaries; but the special peculiarities of the protoplasma boundary layers, and hence of the dependent electromotor phenomena, are determined by the specific metabolic activities of the protoplasm and vary with these activities. It is evident that metabolic destruction and re-formation of surface-films would involve electromotor variations; and in those cases where the filmmaterial is susceptible to chemical alteration (e.g., oxidation or reduction) under the influence of the local electric currents thus arising, the conditions would also be furnished for the processes of spreading and transmission, which are essential to stimulation. Conditions closely resembling in their general features those just defined are in fact realized in the passive iron model and related inorganic systems described above.

\section{NORMAL BIOELECTRIC VARIATIONS OR ACTION-CURRENTS}

The present view, therefore, refers the normal bioelectric phenomena to variations in the phase-boundary

${ }^{x}$ The precise nature and degree of this alteration are unknown; the term "dissolution" may be regarded as indicating an alteration sufficient to deprive the surface layer temporarily of its properties as a membrane, i.e., as a semi-permeable partition. This effect is equivalent to increase of permeability. 
potentials of the polyphasic living system, protoplasm, which in its nature is subject (especially if highly "irritable") to rapid variations of chemical or metabolic activity. Such variations imply corresponding alterations (breakdown, construction, etc.) of those protoplasmic structures, including the interfacial films, whose formation and maintenance depend on this metabolic activity. Apparently during the normal stimulation of any irritable cell, e.g., a muscle cell, the electromotor properties of the cell surface change in such a manner that the surface adopts temporarily properties like those of an injured or altered surface; i.e., one which has lost its semi-permeability. This effect, the result of a metabolic change of some kind, in which oxidations probably play a chief part, is a reversible one; consequently a reversible electromotor variation accompanies it. It is as if the injury-current were temporary, and lasted for only a brief period, whose duration depends on the rate at which a complete surface layer with the original properties can be re-formed. It has already been pointed out that certain forms of injury-current, those caused by $\mathrm{KCl}$ solution, are reversible if the injury is not too extensive. ${ }^{\mathrm{I}}$ On such a hypothesis we may understand why the whole reversible bioelectric variation occupies a definite time, characteristic for each irritable tissue; this time is determined by the tissue's own specific rate of metabolic construction and destruction. The special reaction-velocities characteristic of the

I It is well known that with excessive stimulation of any kind the return of the normal positivity is delayed or incomplete (Hermann and others). All transitions from complete reversibility to irreversibility can be obtained. (Cf. Ebbecke, "Nembranänderung u. Nervenerregung," Arch. ges. Physiol., CXCV [1922], 555; cf. pp. 58I ff.) 
particular living system under consideration thus determine the duration and other special features of its action-currents, and hence also the time-relations of the dependent or correlated phenomena; e.g., velocity of transmission, refractory period, summation, and chronaxie.

The passive iron model again affords a clear and simple illustration of the manner in which rapid electromotor fluctuations can result from changes in the character of the boundary layer between the two chemically interacting phases. When such a wire, immersed in a solution of nitric acid and connected through a voltmeter with an indifferent electrode (platinum wire) also immersed in the acid, is activated, a sudden change of potential (of about 0.7 volt) is observed. With strong acid (6o vols. per cent $\mathrm{I} .42$ acid or stronger) this variation is automatically and rapidly reversed and the metal resumes its former potential within a second or two; this reversal is a result of the re-deposition of the passivating surface-film; the chemical reaction then ceases. Under certain conditions the return of complete passivity may be delayed, or rhythmical fluctuations of potential and chemical activity may occur; the latter phenomenon is frequent in a somewhat weaker acid (between 50 and 55 volumes per cent), and depends upon the alternating formation and dissolution of the passivating film. ${ }^{\mathrm{I}}$ Many striking electrical phenomena, having features which have been regarded as especially characteristic of bioelectric processes, are in fact exhibited by this model. These phenomena show that rapid variations of

${ }^{x}$ For a fuller description of these phenomena cf. my article in Jour. Gen. Physiol. (I920), op. cit., pp. II3-I5. 
potential, with associated chemical effects (shown in the rapid breakdown and replacement of the surfacefilm of oxidation-product), may occur in a three-phase system of relatively simple constitution, when the third phase has the form of a thin chemically alterable film between the other two.

In a chemically reactive and film-partitioned system such as living protoplasm processes of a similar kind are to be expected; such processes would here also necessarily be associated with variations of potential. And conversely, since in all processes of this type the factors controlling the formation and breakdown of the films are mainly electrical, such systems would be influenced in their chemical activity by electric currents passing through them from external sources. This is in fact true both for metallic systems of this type (which include the mercury-peroxide system) and for living protoplasm.

In living organisms variations of electrical potential are associated with physiological activities of all kinds; and for detailed descriptions of the bioelectric phenomena and for a review of the extensive literature reference must be made to the special treatises on electrophysiology. Bioelectric currents have been shown to accompany the following vital processes:I automatic or reflex activity of the central nervous system, rhythmical processes like the heartbeat or the activity of automatic nerve cells, muscular contraction, nervous and other forms of protoplasmic transmission, glandular secretion, stimulation of special sense receptors (retinal currents), the movements

${ }^{2}$ For a more deailed account cf. Garten's article, "Die Produktion von Elektrizität," loc. cit., Igı. 
of plants (Mimosa, Venus' fly-trap), and growth processes; they are probably also associated with celldivision and ciliary movement.

They appear in fact to be as essential a feature of protoplasmic action as the consumption of oxygen or the evolution of $\mathrm{CO}_{2}$. The fact that their rate of development and their rhythm are influenced by changes of temperature in the manner characteristic of chemical reactions $\left(Q_{x_{0}}=2-3\right)^{x}$ indicates their dependence upon the fundamental metabolic processes of protoplasm. Direct proof that the bioelectric rhy thms are accompanied by rhythmical chemical reactions is at present lacking, but there are many indications that this is the case. The evidence is clearest in those instances where the rhythm is slow. Thus the production of $\mathrm{CO}_{2}$ by dividing sea-urchin eggs follows a rhythm which runs parallel with the rhythm of cleavage; ${ }^{2}$ the latter rhythm is accompanied by a parallel rhythm of variation in the physical properties of the egg surface; 3 and this rhythm is almost certainly associated with a variation of potential. ${ }^{4}$ In a certain sense it is self-evident that the energy of the bioelectric currents, as of other organic activities, represents the transformed energy of chemical reactions;

${ }^{x}$ Cf. Piper's results on tortoise muscle (Elektrophysiologie menschlicher Muskeln, Berlin [I9I I], chap. ix, I30). Cf. also the data in Garten's article, loc. cit.; also Lucas, Journal of Physiology, XXXIX (I9o9), 207.

${ }^{2}$ E. P. Lyon, American Journal of Physiology, XI (1904), 52; Science, XIX (I904), 350 .

${ }^{3}$ Cf. my paper on the physiology of cell-division in the Journal of Experimental Zoölogy, XXI (Ig16), 369.

${ }^{4}$ This is indicated by Miss Hyde's observations in the Fundulus egg, American Journal of Physiology, XII (I904), 24i. 
but the problem of the precise nature of the transformation still presents many difficulties.

The special development of bioelectric currents as a means of attack and defense in the electric fishes is a fortunate circumstance for general physiology, since the structural conditions found in the electric organs are full of suggestion for the general theory of the bioelectric processes. These conditions indicate clearly; first, that the powerful effects produced by these organs depend on the summation of the potentials of numerous cellular elements or "disks" (apparently modified muscle cells) arranged in series; and, second, that the action of each single element depends on the alteration of a special and definitely oriented portion of its protoplasmic surface layer. This area is structurally characterized by the rich branching of the nerve-terminals in contact with it; it forms one face of each element, posteriorly directed in the case of the electric eel, while the opposite or anterior face is free from nerve fibers. ${ }^{\mathrm{X}}$ One face of each element is thus innervated and apparently undergoes alteration or activation during activity, in a manner which may be compared with that of an activated gland cell, while the opposite face presumably remains unchanged. The innervated and non-innervated surface layers of adjacent elements thus alternate in position in a manner comparable with the alternation of positive and negative metallic plates in a battery of galvanic elements in series. A closer comparison would be with the original galvanic "pile," where each pair of plates, copper

${ }^{1}$ Cf. Gotch's article in Schafer's textbook, II, 56r, for an account of the essential structure of the electric organ; also Biedermann's Electrophysiology and Bernstein's Eleklrobiologic. 
and zinc, in direct contact with each other, is separated from the next pair in the series by disks of cloth or paper soaked with electrolyte solution. Similarly the innervated and non-innervated surfaces of two adjacent elements are in contact, while the two surfaces of the same element are separated by the mass of internal protoplasm which is modified in a characteristic manner. This arrangement constitutes strong evidence in favor of the theory that the surface-films or plasma membranes of these cellular elements play essentially the same part as the electrodes or metallic plates in batteries. ${ }^{\mathrm{I}}$ In the active electric organ the current of the discharge (positive stream) runs within each cell or element from the innervated to the non-innervated surface, just as in the usual type of bioelectric circuit (e.g., of a single muscle cell) the intracellular direction of the current is from active to inactive (i.e., in the external medium from inactive or "positive" to active or "negative"). Although the E.M.F. of each cellular element is small, apparently the same as that of a single muscle cell (viz., $0.04-0.05$ volt), a high potential between the terminals of the series is attained by means of the summation of many elements. Brünings has shown that by arranging several frogs' muscles in series, with the cut surface of one apposed to the uninjured surface of the next, a summation of potentials may be obtained. ${ }^{2}$ The structure of the electric organ is thus in striking conformity with the theory that the bioelectric currents originate in a manner essentially similar to that

× Compare Bernstein's account in his Elektrobiologie, chap. vi, p. I2 I.

${ }^{2}$ Brünings, Arch. ges. Physiol., XCVIII (I903), 241. According to Brünings, potentials of a volt or more can be obtained by arranging muscles in series. 
of the currents produced by the usual combinations of metallic electrodes and electrolyte solutions (or so-called "batteries"), with the difference that in the living system the electromotor surfaces consist of thin protoplasmic films having a composition and structure which are subject to rapid variation.

The potential changes are relatively small in single cellular elements and their approximate range may be readily determined in parallel-fibered muscles like the frog's sartorius. Here, with a symmetrical side-by-side arrangement of the elements, there can be no summation of potentials; and the conditions are like those of a battery arranged "in parallel." The maximum range of variation during contraction does not usually appear to exceed 0.05 volt, a potential-difference similar to that of the demarcation-current. According to some observers, however, the potential of the action-current in muscle during strong contraction may be greater than that of the demarcation current, and may even attain 0.08 volt. Comparative observations of the demarcation-current potentials throughout a wide range of invertebrate and vertebrate forms give magnitudes of the order of 0.03 to 0.05 volt. ${ }^{\mathrm{I}}$ The exact physico-chemical significance of these values cannot be stated at present. Bernstein has investigated the influence of temperature on the demarcation potential of muscle, and finds that within the physiological range (from $5^{\circ}$ to $30^{\circ}$ ), its magnitude is closely proportional to the absolute temperature, as in the case of electrode or diffusion potentials. ${ }^{2}$

${ }^{x}$ Cf. the data in Garten's article, loc. cit.

2 Bernstein, Arch. ges. Physiol., XCII (1902), 52 I, XXXI (1910), 589; cf. also his Elektrobiologie, chap. v. 
Considering the fact that the potentials as measured must be less than those actually existing in the living tissue-because of the partial cross-circuiting of the current through the fluids of the tissue-it seems probable that in typical active tissues the normal fluctuations of potential in single cells have a range of 50 to roo millivolts. The bioelectric currents are thus weak; their intensity, however, is amply sufficient to excite sensitive tissues, as is demonstrated in the laboratory experiments in which nerves and muscles are excited by demarcation and action currents ("rheoscopic frog" experiments).

In each special instance the duration, the rate of variation, and the rhythm-in the cases where the variations are rhythmical-exhibit special features characteristic of the tissue and of the species. Comparison of the conditions in different tissues and animals reveals the existence of highly significant correlations between the timerelations of the electrical response and of the normal functional response or mode of activity of the tissue. When the bioelectric variation develops rapidly and is of brief duration, the response of the tissue to stimulation is also rapid; e.g., in a muscle the duration of the latent period and of the single twitch is brief, the chronaxie is also brief, and the propagation of the excitation-wave is rapid; the refractory period and the summationinterval are also brief. On the other hand, tissues with slowly developing bioelectric variations exhibit a slower rate of response and a slower subsidence of their activity; the muscular twitch, the chronaxie, the refractory period, and the summation-interval are relatively prolonged and the transmission is slow. 
Changes of temperature influence the functional processes and the bioelectric processes similarly. For example, in a special study by Keith Lucas, ${ }^{\mathrm{r}}$ in which the temperature-coefficient of the rate of development of the bioelectric variation in the frog's sartorius was compared with that of the propagation-velocity of the excitation-wave, almost identical values were found for the two processes. In a typical experiment, the time required for the rise of the bioelectric variation from zero to its maximum at $8^{\circ}$ was .004 I of a second, and at $\mathrm{I} 8^{\circ}, .0024$ of a second; the ratio of these two values, r.64, was almost identical with that of the propagationvelocities of the excitation-wave (contraction-wave) at the two temperatures. In other words, change of temperature influences the rate of protoplasmic transmission in the same manner as it influences the rate of variation of potential.

It seems clear that the bioelectric variations are inseparably connected with chemical or metabolic processes in the living cells, and that the characteristic rate at which the tissue reacts and conducts excitation is a direct function of the rate of both processes. This rate is determined by the specific chemical and structural constitution of the tissue as well as by external factors. such as temperature and the state of the surrounding medium. The evidence already reviewed indicates that the essential changes underlying the bioelectric phenomena occur at the cell boundary; hence, these phenomena may be regarded as an index of chemical decompositions or other reactions occurring in the protoplasmic surface-films. Apparently these reactions

I Journal of Physiology, XXXIX (rgog), 207. 
change the composition of the films and alter their electromotor and other properties (permeability, physical consistency, etc.), and the bioelectric currents are the result.

The question of whether the bioelectric currents stand in the relation of cause or of effect to the other physiological activities of the cell is not one to be answered simply, since, as in so many other natural processes, the relations are of a reciprocal kind. Apparently the conditions are of the same general physicochemical nature as in any reversible type of galvanic cell (storage battery); a current from an outside source traversing the system may be the means of inducing definite chemical reactions in the latter; or the system may by its own spontaneous chemical action generate an electric current which traverses the surroundings and there produces the usual effects of such currents. Similarly, the electric variation of a cell or nerve fiber may be an accompaniment or effect of other processes, presumably chemical, in the living protoplasm; but once having arisen, a bioelectric current may act in the same manner as any other electric current and influence secondarily other processes in the electrically sensitive living system. Thus there is every evidence that the electric factor, as such, determines the transmission of excitation from one region to another of a conducting nerve fiber or other excitable protoplasmic system; and that the characteristic rate of transmission is determined by the rate at which the local variation of potential rises from zero to its full value. ${ }^{\mathrm{I}}$ It is evident that if

${ }^{3}$ Cf. my article in American Journal of Physiology, XXXIV (I9r4), 4I4. 
each active region excites electrically the adjoining inactive region, by means of the local bioelectric current accompanying activity, the velocity with which excitation is transmitted from region to region will be higher the more rapidly this current develops. Lucas' observation just cited shows in fact a close parallelism between these two rates, in the same tissue at different temperatures. Variations in transmission-velocity in different tissues, and in the same tissue under different conditions, would on the foregoing hypothesis have a direct causal dependence on the rate of change of potential characteristic of the tissue.

Reference has already been made to the fact that in each species of animal the bioelectric variations of the active tissues have specific peculiarities - of rate of development, normal range, duration, rhythm, etc.-which exhibit a close correspondence with the peculiarities of function and activity characteristic of the species. For example, the normal bioelectric variation of a special organ like the heart is an accurate index of the normal rate and sequence of its different processes; hence, the electrocardiogram may be a delicate means of detecting abnormalities in the action of this organ. Presumably a tracing of the bioelectric variations from the group of muscles involved in the act of speech could, with sufficient knowledge and analytical skill, be translated into the actual words uttered. From what has already been said it will be obvious that this close correspondence between the functional activity of a living system and the character of its bioelectric variations implies a similar correspondence of both with the underlying variations of metabolic activity. 
The range of the electric variations obtained from an isolated muscle which is made to contract in a graded manner by single stimuli of different strengths shows a direct correlation with the height of contraction, but the significance of this fact is not obvious. It is possible (I) that the single elements or cells give actioncurrents of varying intensity, or (2) that each element has a constant variation, but that the number of elements excited varies. The probabilities favor the latter alternative; in certain tissues such as heart muscle, the electric variation exhibits a constant range which is independent of the intensity of the stimulus; the whole tissue responds with a complete contraction to any sufficient stimulus - an example of the "all or none" behavior-and correspondingly the range of the electric variation is constant. There is good evidence that in normal unfatigued voluntary muscle the variations in the strength of contraction depend on the number of cells contracting, and not on variations in the degree of contraction of single cells. ${ }^{\mathrm{I}}$ Similar considerations apply to the bioelectric response, which in the single elements of this tissue and of nerve appears also to exhibit the "all or none" character. Apparently to any constant manifestation of normal physiological activity a constant bioelectric variation corresponds.

On the other hand, under certain abnormal conditions the bioelectric variation, e.g., in heart muscle, may continue without the normally associated contraction; ${ }^{2}$

${ }^{x}$ Cf. Lucas, Journal of Physiology, XXX (1905), I25; XXXVIII (1909), II3; F. H. Pratt, American Journal of Physiology, XLIV (1917), 517; Pratt and Eisenberger, ibid., XLIX (I9I9), I.

${ }^{2}$ Noyons, K. Akad. Wet., Amsterdam (Nov., 1908 and April, r9ı) (cited in Lucas' Croonian Lecture, "The Process of Excitation in Nerve and Muscle," Proceedings of the Royal Society, B, LXXXV [19I2], 512). 
in such cases the internal contractile mechanism of the cell is incapacitated; and apparently this may occur without disturbing the primary processes of stimulation and conduction, which depend on surface-changes associated with the bioelectric variation. Such a dissociation of conduction and excitation from contraction is also seen during the water-rigor of muscles; at a certain stage of water-rigor a muscle will conduct excitation without contracting. ${ }^{x}$ Under normal conditions, however, the electromotor variation and the functional process exhibit close parallelism. The inverse type of case, i.e., where a muscle contracts or nerve conducts without exhibiting a bioelectric variation, does not seem to occur. ${ }^{2}$ The electromotor variation seems to be inseparable from the process of stimulation. It may be prevented from appearing (by anæsthesia, etc.). but in that case all of the other manifestations of stimulation are also prevented. Such facts again indicate the primary and controlling rôle of the electromotor iariations in cell-activities.

\section{TIME RELATIONS OF BIOELECTRIC VARIATIONS}

We have seen that the time occupied by a single electromotor variation (i.e., of the unsummated effect resulting from a single stimulus) varies characteristically

× Biedermann, Sitzungsberichte der Akademie, Wien., XCVII (ıSSS), Part III, p. roI; cf. also Overton, Arch. ges. Physiol., XCII (1902), 1 40 ; he finds that frog's muscle immersed in 0.2 per cent $\mathrm{NaCl}$ loses contractility while still retaining irritability and conductivity. Cf. also Härtl, Engelmann's Archiv f. Physiol. (1904), p. 8o. Robertson has observed the same phenomenon in the intestine of the Australian blowfly after bathing in $\mathrm{CaCl}_{2}$ solution (Ergebnisse der Physiol, $\left.\mathrm{X}[1910), 305\right)$.

${ }^{2}$ Cf. Lucas' discussion of this question in his Croonian Lecture, op cit., p. 502. 
from tissue to tissue. This time is exceedingly brief in rapidly responding tissues like voluntary muscle and nerve; for example, in frog's muscle the "electrical response" has a shorter latent period and a much shorter duration than the "mechanical response"; the muscular twitch begins (at $20^{\circ}$ ) about 0.01 of a second after stimulation and lasts about o. I second, while (according to Snyder) the latency of the electric variation is about 0.003 of a second and its total duration about 0.007 of a second. ${ }^{I}$ Thus the electric variation may be completed before the muscle has begun to contract; it is the first evident effect of stimulation and apparently is an index or accompaniment of critical changes which determine the succeeding chemical and mechanical processes.

In general the more rapidly a muscle contracts the briefer is its chronaxie and the more rapidly its bioelectric variation develops. ${ }^{2}$ There is also a direct correlation between the rapidity of contraction and the rapidity of transmission of the excitation-wave; this is true not only for the transmission in the muscle itself, but also for the transmission in the motor nerve supplying the muscle. ${ }^{3}$ Rapidity in physiological action thus implies rapidity in the associated bioelectric processes. A muscle and its motor nerve constitute a single reactionsystem, and the rate of the bioelectric processes is a close index of the rate of reaction of the entire system. Transmission of the excitation-state from nerve to muscle through the motor end-plate is apparently a phenomenon of the same kind as transmission from region to region

${ }^{x}$ Cf. C. D. Snyder, American Journal of Physiology, XXXII(19r3), 336.

${ }^{2}$ Cf. Lapicque, Jour. de physiol. et de path. gên., X (rgo8), 6or.

3 Carlson, American Journal of Physiology, X (I904), 40I; XV (Ig06), I36. 
along the same element. Hence if transmission is a case of secondary electric stimulation by the current of the local circuit, it is clear that rapidity of electromotor variation, implying rapidity of conduction, is necessary in a nerve which excites a muscle by means of the bioclectric circuit formed across the junction between nerve fiber and muscle cell. Lucas has furnished evidence that the motor end-plate in vertebrate muscle has a special chronaxie differing from that of either the nerve fiber or the muscle cell, ${ }^{x}$ but it does not appear that the conditions determining the transmission between nerve and muscle are essentially altered by the presence of this intermediary element. According to Lapicque the blocking action of curare results from an alteration (slowing) of the chronaxie of the end-plate. ${ }^{2}$ Any two contiguous elements, one of which is excited by the bioelectric variation of the other, must have similar time-factors of excitation; dissimilarity in the timefactors or "heterochronism" (to use Lapicque's term) would be inconsistent with such transmission. This conclusion is a simple corollary of the general laws of electric stimulation described above; a current traversing an irritable element must have more than a certain duration and rate of change, or it fails to stimulate. Similarly, the momentary current at the myoneural junction, when the excitation-wave traveling along the nerve reaches that region, must have a duration and rate of change corresponding with the chronaxic of the muscle cell.

'Lucas, Journal of Physiology, XXXVI (1907), I 3 .

${ }^{2}$ Lapicque, Compt. rend. soc. biol., LXXII (19r3), 674. Cf. however, the critique by Boruttau in Zentr. Physiol., XXXI (1916), 303. 
In the following table $\mathrm{e}^{\mathrm{x}}$ are collected a considerable number of observations showing the rate of development

Tissue

A. Striated Muscle:

Frog's

gastrocnemius...

Frog's

Duration of Rising

Phase of Action-Current

Curve $(\sigma=.001$ sec.) Velocity of Propagation
of Excitation-Wave

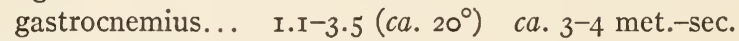

Frog's sartorius ... I.6-3 $2\left(\mathrm{ca} .20^{\circ}\right)$

Frog's sartorius... 4. $4-4.2\left(8^{\circ}\right) \quad$ ca. I.2 met.-sec. $\left(8^{\circ}\right)$

Frog's sartorius ... 2. 2.4-2.9 (I $\left.8^{\circ}\right) \quad c a .1 .65$ met. - sec. (I $8^{\circ}$ )

Frog's sartorius . . . I3 $\left(3^{\circ}\right)$

I.06 met. - sec. $\left(3^{\circ}\right)$

Frog's sartorius . . . $5.8\left(14.8^{\circ}\right)$

r. 65 met. - sec. $\left(14.8^{\circ}\right)$

Frog's hyglossus. . . $20\left(3^{\circ}\right)$

0.38 met. - sec. $\left(3^{\circ}\right)$

Frog's hyglossus... $8.9\left(14.7^{\circ}\right)$

Mammalian muscle ca. 0.96 met.-sec. (14. $\left.7^{\circ}\right)$

Rabbit's

gastrocnemius... ca. $2\left(\mathrm{ca} .37^{\circ}\right)$

B. Nerve:2

Frog's sciatic..... 0.9-I.2 (ca. I $\left.8^{\circ}\right)$

Frog's sciatic..... $0.55\left(32^{\circ}\right)$

Rabbit's sciatic... ca. $0.5\left(32^{\circ}\right)$

Dog's sciatic.......ca. $0.7\left(36^{\circ}\right)$

Io-1 3 met.-sec. in man's forearm

I From my article, American Journal of Physiology (I9I4), loc. cit.; the observations cited are from many different authors; for complete references $\mathrm{cf}$. this article.

${ }^{2}$ These observations were made with the thread galvanometer. More recent work on vertebrate nerve with other methods indicates that the rise is even more rapid. The rates shown by the recent work of Gasser and Erlanger with the cathode ray oscillograph are almost double those indicated by Garten's observations cited in the table. Cf. American Journal of Physiology, LXII (I022), 5I7. See also the work of $\mathrm{R}$. Plaut with a rheotome method; in the frog's sciatic the rise was estimated at 0.2 to $0.3 \sigma$ (Z. für Biol., LXXVIII [I923], I33). 
Tissue

Non-medullated

(splenic of horse) $c a .60-70$

Olfactory of pike.. ca. $70\left(12^{\circ}\right)$

Commissural of anodonta...... ca. 200

Mantle-nerve of octopus...... 8.2-II.3

Mantle-nerve of octopus....... ca. 20

C. Cardiac Muscle:

Ventricular muscle of mammal..... Io-I5 (body temperature)

Ventricular of frog. $\quad 40-60\left(\mathrm{ca} .18^{\circ}\right)$

D. Smooth Muscle:

Retractor penis of $\operatorname{dog} . . . . . . . . c a .2 \mathrm{sec}$.

Ureter muscle.... $\quad 0.2-0.4 \mathrm{sec}$.

E. Influence of Narcotics:

Frog's sciatic.... Normal: $32-4$ $\left(8.9^{\circ}\right)$

Partly narcotized: $3 \cdot 4^{-4 \cdot 4}$ Normal: ca. 4,; narcotized. 5.2-6

Pike's olfactory ... Normal: 55-60 $\left(\mathrm{ca} .9^{\circ}\right)$; partly narcotized: $67-82$
Velocity of Propragation of Excitation llave

cu. $0.47-0.54 \mathrm{met}-\mathrm{sec}$. 60-90 mm. -sec. $\left(5^{\circ}\right)$ I I $8-150 \mathrm{~mm} .-\mathrm{sec} .\left(13^{\circ}\right)$ I $60-2.40 \mathrm{~mm} .-\mathrm{sec} .\left(20^{\mathrm{I}}\right)$

ca. $2.5 \mathrm{~cm} .-\mathrm{sec}$.

(Varying estimates from I to $5 \mathrm{~cm}$. $-\mathrm{sec}$.)

2.5-3.5 met. sec. in O. vulgaris

ca. 2 met.-sec. in $\mathrm{O}$. punctatus

Averages apparently 2-4 met. $-\mathrm{sec}$.

From 50-200 mm.-sec.

I-7 mm sec.; average ca. $5 \mathrm{~mm}$.-sec.

Average $c a$.

I. - I $5 \mathrm{~mm}$. $-\mathrm{sec}$.

Average 17.3 met.-sec. in normal; $c a$. I 2 met.-sec. in weakly, $9 .+$ met. - sec. in more strongly narcotized nerves

Average normal velocity: ca. SI mm.-sec.; in narcosis $\mathrm{Ca}$. $5^{0}$ mm.-sec. 
of the local bioelectric variations, as related to the transmission-velocity of the excitation-wave in a variety of vertebrate and invertebrate tissues under different conditions. It will be noted that cold, anæsthesia, and fatigue, which retard the rise of the bioelectric variation, also retard the speed of propagation in about the same proportion. The general correspondence shown seems to leave no doubt that a direct correlation exists between the respective rates of development of the local electric processes and the velocities with which excitation is transmitted from region to region in the different conducting tissues.

This fact, taken by itself, may seem equivocal in its significance, since it is evident that any wave of alteration occupying a definite length of the conducting element and associated with a change of potential would, as it passed one of the electrodes of a recording instrument (e.g., a string galvanometer), cause an excursion, the rate and duration of which would depend on the speed of the wave. The electric variation might thus conceivably be simply a sign or index of the passage of a wave of activation, without having any causal relation to the process of transmission. Other evidence, however, to be considered below, indicates that the electric variation, as such, is the main factor determining the transmission of excitation from the active region to the adjoining resting region (see chap. $\mathrm{xv}$ ).

It is significant that many normal bioelectric processes, e.g., those accompanying muscular contraction or nervous activity, as they occur under physiological conditions in the intact organism, are typically rhythmical. The underlying chemical reactions must therefore 
also be rhythmical, and if these reactions are primarily' those occurring in the protoplasmic surface-films, it follows that rhythmical variations in metabolic activity are characteristic of this region of the cell. There are various general facts indicating a tendency to rhythm in the activities at free cell-surfaces; for example, the wide distribution of such phenomena as ciliary movement, in which protoplasmic surface-processes show a regular mechanical rhythm which is presumably accompanied by a chemical and electromotor rhythm. The filamentous processes formed under certain abnormal conditions from the surface of simple cells like blood corpuscles also often exhibit rhythmical movements. ${ }^{{ }^{3}}$ All such movements are probably of an electro-capillary nature, and referable to general conditions similar to those determining the rhythmical phenomena in the polyphasic inorganic systems (mercury in hydrogen peroxide, iron in nitric acid) described above. As already seen, variations in the structure and composition of thin interfacial films are the essential factors in all such phenomena.

The rhythmical bioelectric variations accompanying the normal innervation of muscle have been investigated in much detail since the application of the thread galvanometer to physiological uses by Einthoven. In man, Piper found the rhy thmical action-currents obtained from single voluntary muscles (e.g., extensor of forearm) to exhibit a remarkable constancy of rhythm, of about

${ }^{x}$ Cf. Kite, Journal of Infectious Diseases, XV (1914), 319; Oliver, Science, XL (19r4), 645 .

The minute precipitation-filaments first formed when an iron wire is placed in ferricyanide solution frequently exhibit rhythmical movements of a kind suggesting ciliary movement; cf. Biological Bullectin, XXXIII (1917), I39. 
fifty per second. ${ }^{I}$ Records taken from other muscles showed similar but not always identical rates, varying from forty per second in the thigh muscles to sixty per second or more in the jaw muscles; and it appears probable that under normal conditions different muscles have characteristic differences in their electromotor rhythms. There is every evidence that the muscle rhythm corresponds to the electromotor rhythm of innervation, which in turn corresponds to the rhythm of discharge from the nerve cells in the central nervous system. Numerous observations since Helmholtz' time have shown that a voluntary muscle during contraction emits a low musical note, apparently indicating a rhythmical variation in mechanical tension; and it has been shown by stimulating the nerve rhythmically, by tetanizing currents of known frequency, that the note derived from the muscle has a pitch corresponding with the rhythm of innervation, up to a frequency of several hundred per second. ${ }^{2}$ The same is true of the rhythm of the electric variation obtained from the muscle during rhythmical innervation; in the frog's muscle the rhythmical electromotor variations show the same frequency as that of the stimulus, until an upper limit of about I 50 to 200 per second (at room temperature) is reached; above this limit the electric variations of the muscle are less frequent than those of the nerve, becoming irregular with the higher frequencies. ${ }^{3}$ In the case of

${ }^{\mathrm{I}} \mathrm{H}$. Piper, Elektrophysiologie menschlicher Muskeln, Berlin (I9I2); cf. chap. vii.

${ }^{2}$ Cf. Piper, op. cit., chap. $\mathrm{x}, \mathrm{p}$. I43, for a fuller account and references to literature.

${ }^{3}$ Cf. F. Buchanan, Journal of Physiology, XXVII (I9०I), 95; Hoffmann, Archiv Anat. u. Physiol. (1909), p. 430; also Judin, Arch. ges. Physiol., CXCV (1922), 527 . 
warm-blooded animals the synchronism between innervation and the electromotor response of the muscle continues up to frequencies approaching $\mathrm{I}, 000$ per second. Limits are set to the possible rate of electric rhythm by the refractory period of the muscle cells. Recently: Gasser and Newcomer, using an amplifying arrangement, have shown that in the normal innervation of the dog's diaphragm, the electromotor rhythm of the phrenic nerve corresponds' exactly with that of the muscle; every electromotor wave in the muscle appears to be produced by a corresponding one in the nerve; the rhythms observed varied between 72 and 104 per second. ${ }^{2}$ It is thus clear that the normal rhythm of excitation in the muscle cells depends on the rhythm of innervation; in the intact organism the latter rhythm is determined by the special rate of rhythmical discharge characteristic of the motor nerve cells; ${ }^{3}$ and this rhythm, under the usual precisely regulated physiological conditions, is remarkably regular.

Experiments of Piper on the influence of temperature on the natural bioelectric rhythm in the tortoise have shown that the temperature-cocfficient of the rhythm is of the usual order of chemical reaction-velocities. The following frequencies of oscillation per second were observed by him in a string galvanometer connected with the retractor muscle of the neck, which was caused to contract reflexly at different temperatures (see p. 334).

${ }^{2}$ Cf. Höber, Arch. ges. Physiol., CLXXvil (1919), 305.

${ }^{2}$ Gasser and Newcomer, American Journal of Physiology, LIII (1921), I.

${ }^{3}$ Cf. C. Foa, Z. allg. Physiol., XIII (Igr I), 35.

4 Piper, Arch. Anat. u. Physiol (Physiol. Abthcil.) (1910), p. 207; also Elektrophysiologie menschlicher.Muskeln, chap. v, p. ${ }_{3} 0$. 
The normal tetanic contractions of voluntary muscles are thus summated contractions resulting from rhythmical innervation. We can thus understand why the summated contraction resulting from artificial rhy thmical electrical stimulation of the muscle is physiologically indistinguishable from the normal contraction; in reality the natural as well as the artificial tetanus is a result of rhythmical electrical stimulation. Whether the initial electric disturbance in the muscle cell originates

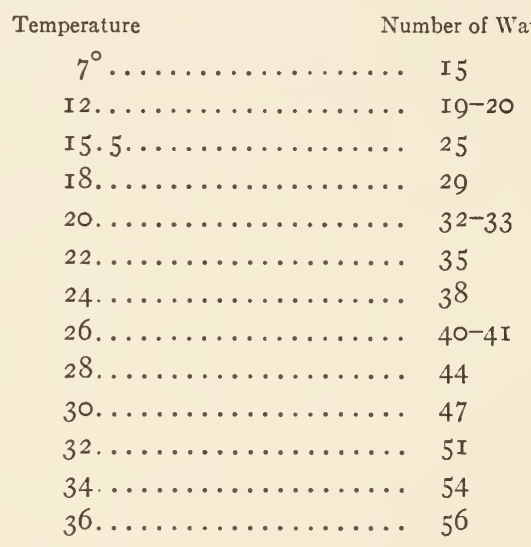

at the motor end-plate or at the point of entrance of a current from an external stimulating electrode is a matter of indifference, so far as the response of the living tissue is concerned. In the intact organism under normal conditions the rate of rhythm is fixed or predetermined by the constitution of the motor cells in the nervous system. These cells, however, are subject to influence by external conditions, such as temperature or the composition of the surrounding medium (e.g., H-ion concentration), so that many physiological rhythms are 
subject to variation in frequency. Such variations offer some of the most beautiful examples of physiological adjustment to the varying needs of the organism; e.g.. the respiratory rhythm of warm-blooded vertebrates.

The normal rhythm of electromotor discharge may be simple, i.e., there may be a regular succession of single impulses, as in the sinus region which controls the vertebrate heart beat; in this case both the unsummated character of the muscular contraction and the form of the galvanometric record show that in the sinus, auricle, or ventricle a single electromotor variation corresponds to each beat. In other cases, however, a rhythmical succession of discharges may occur, each discharge being itself rhythmical; this is the case, for example, in the nerve cells innervating the respiratory muscles of vertebrates. Garrey has recently observed a similar condition in the Limulus heart; 'I corresponding to each beat there is an oscillatory electrical variation or discharge, undoubtedly originating in the ganglion, each discharge exhibiting a constant number, about twelve, of separate electromotor variations. The series or "volley" of secondary waves or pulses forming each discharge has its own rhythm, which is independent of the cardiac rhythm as a whole. Change of temperature changes the rate of oscillation in each ganglionic "beat," but the number of distinguishable oscillations always remains about twelve. It appears to be a general rule that the electromotor rhythms are influenced by temperature in the same manner as most other physiological rhythms, i.e., show the chemical temperature coefficient;

I W. E. Garrey, unpublished observations at Marine Biological Laboratory, Woods Hole, Massachusetts. 
their intimate connection with the metabolic reactions of the living protoplasm is thus indicated.

In the frog's muscle the rhythmical character of the bioelectric current during tetanic contraction is most readily demonstrated by the "secondary tetanus" experiment, in which a muscle with its nerve laid along another muscle is thrown into tetanus when the second muscle is tetanized. In man, the slight mechanical oscillation accompanying steady voluntary contraction of the arm muscles can be demonstrated by means of the hot-wire sphygmograph; this mechanical rhythm has the same period as the electromotor rhythm; i.e., about fifty per second. ${ }^{\mathrm{T}}$ This experiment is especially interesting as indicating (as do also the experiments with the string galvanometer) that the various nerve cells inervating a muscle discharge synchronously or in phase with one another. The experiments of Gasser and Newcomer also indicate that this is true of the nerve cells innervating the opposite halves of the mammalian diaphragm. ${ }^{2}$ Apparently the several nerve cells constituting each motor group are under some control by which all are impelled to react synchronously, or "keep time." This suggests a co-ordination dependent on some rapidly transmitted influence, presumably electrical; the synchronous activity of spermatozoa when gathered in clumps, or of ciliated cells, seems to be a phenomenon of a closely related kind (see p. 392).

x A. V. Hill, Journal of Physiology, LV (1921), Proceedings of the Physiological Society, p. I4.

${ }^{2}$ Gasser and Newcomer, op. cit., p. 24. 


\section{CHAPTER XIV}

\section{MEMBRANE CHANGES DURING STIMULATION}

REFRACTORY PERIOD

We have already given reasons for regarding the bioelectric variations as a result of structural change, controlled by metabolic change, in the protoplasmic surface layers. From this point of view another constant accompaniment of the stimulation-process, the refractory period, receives a consistent theoretical explanation. The presence of the intact surface-film is necessary for stimulation; if the film is broken down as a consequence of stimulation, it must be re-formed and restored to its original state before a second complete stimulation is possible. This deduction is in agreement with our general experience of stimulation-processes. It is a striking fact that in all irritable tissues stimulation is immediately followed by a period of insensitivity and subnormal irritability; this period of temporary depression, which is extremely brief in rapidly responding tissues, is the refractory period, and there is evidence that it corresponds to the period during which the film is undergoing breakdown and reconstruction. During the refractory period the tissue loses at the same time both its susceptibility to electric stimulation and its ability to transmit states of excitation; in other words, there is a temporary loss of both irritability and conductivity. ${ }^{\mathrm{I}}$

${ }^{2}$ Cf. Lucas and Bramwell, Journal of Physiology, XLII (1911), 495; Adrian, ibid., L (1916), 345 . 
The duration of the refractory period varies greatly in different irritable tissues, and under normal conditions is specific for each tissue. The most significant general correlation is that it is brief in tissues with brief chronaxie, and vice versa. As already pointed out, in any irritable tissue the length of the chronaxie is closely related to the duration of the single bioelectric variation; and the duration of the refractory period shows a similar correlation. This parallelism has frequently attracted attention, and its significance has recently been discussed in considerable detail by Tait, ${ }^{\mathrm{I}}$ who has reached the conclusion that the first part of the period, the interval of complete inexcitability or "absolute refractory period," which is of brief duration, corresponds with the period of upstroke of the curve of electromotor variation, while the "relative" part of the period corresponds with the downstroke or return phase. Tait has shown that the relative refractory period is greatly prolonged by drugs (yohimbine, protoveratrine) which retard the return phase of the bioelectric variation. In general, he finds the return of irritability to run parallel with the return of the normal resting potential of the muscle.

While it seems clear that the delayed recovery in such cases has a connection with the delay in the recovery of the normal electromotor properties of the tissue, the correlation is apparently not a simple one. More recent evidence shows that under normal conditions the relative refractory period in muscle and nerve may last several times longer than the return phase of the electric variation. $^{2} \quad$ The curve of the latter is very nearly symmetrical

× Tait, Quarterly Journal of Experimental Physiology, III (I9ro), 2I I.

${ }^{2}$ Cf., for heart muscle, Trendelenburg, Arch. ges. Physiol., CXLIV (I9I2), 39; for nerve, Adrian, Journal of Physiology, XLVIII (I9I4), 453. 
in unfatigued tissues, while the change in irritability follows a markedly asymmetric course; i.e., there is a rapid and complete loss of irritability on stimulation, followed by a relatively gradual recovery. Evidently: in the recovery process additional factors enter which are independent of the bioelectric variation. It is, however, highly significant that in all cases irritability seems to disappear completely during the rising phase of the variation. This partial correlation between the timerelations of the two phenomena indicates that the conditions determining the electromotor variation are closely connected with those determining the temporary loss of irritability.

The division of the whole refractory period into two distinct subperiods, known respectively as "absolute" and "relative," is its most interesting feature. The absolute period is the brief interval of complete insensitivity immediately following a single effective stimulus. During this interval a second stimulus, no matter how strong, has no appreciable effect. It is as if the tissue for a brief time lost its irritability completely; hence, two stimuli succeeding each other within this interval produce the same effect as a single stimulus; while if the second is sent in after the completion of this brief interval, its effect is seen in an increased response or summation-effect. The completely inexcitable period in a frog's motor nerve at $20^{\circ}$ lasts about 0.00 I to $0.00 \mathrm{I}_{5}$ of a second; ${ }^{x}$ in the sartorius muscle it is from two to three times longer; and in both of these cases its duration is closely similar to that of the upstroke of the bioclectric

${ }^{2}$ Cf. Adrian, Journal of Physiology, XLVIII (19r4), 453; L (1910), 345 . 
variation. ${ }^{\mathrm{I}}$ It is followed by a second period, the relative refractory period, during which irritability returns progressively to normal; this interval has several times the duration of the absolute refractory period, and in nerves under certain conditions (increased $\mathrm{H}$-ion concentration) it may be followed by a brief period of supernormal excitability. ${ }^{2}$ In cardiac muscle the return of normal excitability is relatively very slow, even when the increased duration of the bioelectric variation is taken into account; in this tissue the period of complete inexcitability appears to outlast the entire bioelectric variation, and its limits do not seem to be very clearly defined; at $15^{\circ}$ Lucas found its duration to be somewhat more than 0.4 of a second. ${ }^{3}$ Usually it has been supposed that the regular and somewhat slow rhythm characteristic of this tissue is dependent on its long refractory period, which is almost equal in duration to the period of muscular relaxation. A prolonged refractory period is also characteristic of the nerve cells controlling other physiological rhythms of slow period, such as those of certain motor reflexes in higher vertebrates (e.g., scratch reflex in the dog).

In any given tissue the duration of the refractory period is influenced by the chemical conditions in the surroundings as well as by the physiological state of the tissue and the temperature. It is lengthened by fatigue,

I This is apparently strictly true of frogs' voluntary muscle, but in nerve Adrian finds its duration somewhat longer, equal to that of the whole bioelectric variation. In cardiac muscle it may be still longer. Cf. Adrian, Journal of Physiology, LV (192I), I93.

${ }^{2}$ Adrian, Journal of Physiology, L (1920), I.

${ }_{3}^{3}$ Lucas, Journal of Physiology, XLI (Igro), 368; cf. pp. 383-84. 
lack of oxygen, and partial ani'sthesia, at least in some cases; ${ }^{r}$ but in nerve Lucas found the rate of recovery to be normal in solutions of alcohol sufficient to greatly retard transmission. ${ }^{2}$ Bazett observed an influence of the salts of the medium, increase of potassium lengthening, and increase of calcium shortening the interval. ${ }^{3}$ Various poisons such as veratrine, muscarine, digitalis, and barium salts also lengthen the refractory period. ${ }^{4}$

The temperature-coefficient appears to be high in all cases. In frog's muscle and nerve Bazett and Adrian obtained $Q_{\text {ro }}$ values of three or more..$^{5}$ Burdon-Sanderson's observations on the frog's heart indicate values ranging from 2 to $2.5 .{ }^{6}$ The fact that the process of recovery shows this high coefficient seems to indicate its dependence upon processes of constructive metabolism: the temperature-coefficients are in fact similar to those of growth processes.

It is interesting to note that the various forms of protoplasmic transmission (in nerve, muscle, etc.), which theoretically depend on processes of breakdown, have usually shown temperature-coefficients of a dis-

${ }^{x}$ Cf. Verworn, Allg. Physiol., 4th ed., Jena (1903), p. 559; Irritability, Yale University Press, Ig13, chap. xii; Fröhlich, Z. allg. Physiol., III (1904), 468.

${ }^{2}$ Journal of Physiology, XLVI (1913), 470.

3 Bazett, Journal of Physiology, XXXVI (1908), 414.

${ }_{4}$ Cf. Tait, loc. cit.; Trendelenburg, loc. cit.; de Boer, Journal ef Physiology, XLIX (1915), 312; American Journal of Physiology, XLIIII (I921), I79, I89.

${ }_{5}$ Cf. Bazett, loc. cit.; Adrian (1914), and (1921), loc. cit.

${ }^{6}$ Eckstein (Arch. ges. Physiol, CLxxxili $[1020]$, 40) finds a value of 2.6 for the refractory period of frogs' ventricle (average of 10 experiments between $5^{\circ}$ and $20^{\circ}$ ). 
tinctly lower order $\left(Q_{\mathrm{IO}}=\mathrm{I} \cdot 5^{-2}\right)$. $^{\mathrm{x}}$ The significance of this fact is not altogether clear, but it suggests that purely physical factors play more part in the destruction than in the reconstruction of the surface-film. There are general reasons for expecting that a process of structural breakdown, in which purely physical factors predominate, will have a low temperature coefficient; accordingly, since protoplasmic transmission is apparently dependent on such a breakdown, it is not surprising that its temperature-coefficient should be lower than that of the recovery process, which presumably is chiefly a result of metabolic reconstruction. Let us suppose that the first stage in the local excitation-process consists in a removal or destruction, by chemical reaction, of those constituents of the plasma membrane which are responsible for its semi-permeability and coherence; and that the second stage, immediately following, is some purely physical process of disintegration or falling apart, in which diffusion-processes are the chief factor. Then the chemical temperature-coefficient will be shown by the first stage only, while the second and probably longer stage will have the coefficient of diffusion-processes. The whole process will then have a low temperaturecoefficient similar to that observed, as the following simple calculation shows.

We assume that the total period of breakdown at $20^{\circ}$ has a duration of $3 \sigma$ (about the duration of the rising

$\therefore$ For the temperature coefficient of the nerve impulse cf. Maxwell, Journal of Biological Chemistry, III (1907), 359; Snyder, American Journal of Physiology, XXII (1908), I79; Harvey, Carnegie Institute Publications, CXXXII (1910), 35; Lucas, Journal of Physiology, XXXVII (I908), II2. For the transmission of the contraction wave muscle cf. Woolley, Journal of Physiology, XXXVII (I908), I22; Lucas, ibid., XXXIX (1909), 207. 
phase of the bioelectric variation in frog's voluntary muscle), and that of this period one-third ( $\mathrm{I} \sigma$ ) is occupied by the initial chemical decomposition $\left(\mathrm{C}_{20^{\circ}}\right)$, with $\mathrm{Q}_{10}=3$, and the remaining two-thirds $(2 \sigma)$ by a physical disintegration $\left(\mathrm{P}_{20^{\circ}}\right)$, with $\mathrm{Q}_{10}=\mathrm{I} .3$ (the temperaturecoefficient of diffusion processes). Then: (I) Total duration of breakdown at $20^{\circ}, \mathrm{C}_{20^{\circ}}+\mathrm{P}_{20^{\circ}}=3 \sigma$; (2) total duration of breakdown at $10^{\circ}, \mathrm{C}_{10^{\circ}}+\mathrm{P}_{10^{\circ}}=\mathrm{I} \sigma \times 3+2 \sigma \times$ $\mathrm{I} .3=5.6 \sigma$. The ratio $\frac{5.6 \sigma}{3 \sigma}$, is $\mathrm{r} .9$, the $\mathrm{Q}_{10}$ for the total process.

This value is similar to those obtained by a number of investigators (Maxwell, Lucas, Woolley, Snyder, and Harvey) ${ }^{\mathbf{r}}$ for the transmission-process in nerve and muscle. The recovery-process, on the other hand. which apparently occupies the greater part of the refractory period, presumably depends chiefly upon the reconstruction of the surface-film by metabolic synthesis, and accordingly exhibits the high temperature-coefficient characteristic of chemical processes.

The general theory of the refractory period is thus closely related to that of the stimulation-process as a whole. If stimulation is in fact dependent on an alternate breakdown and reconstruction of the surfacefilms of the irritable elements, we should expect irritability (which depends on the state of the film) to vary during the successive stages of the stimulation-process in a manner very similar to that which we observe. Evidently there are two distinct processes involved in the local change of stimulation, corresponding respectively to the "absolute" and the "relative" periods. The

Loc. cit 
view has long been entertained (by Hering and others) that the rising phase of the bioelectric variation is coincident with a period of breakdown of living material, and the return phase with its reconstruction. ${ }^{x}$ In Tait's experiments just described, the delay in the return phase of the variation was found to be associated with a delay in the recovery of normal irritability and conductivity; hence his proposal to identify the relative refractory period with the period of recovery or reconstruction of which the return bioelectric variation is the index. There are, however, various facts indicating that the return variation and the recovery of irritability may vary independently. Trendelenburg has shown that in cardiac muscle excitability does not return until some time after the bioelectric variation is completed; poisoning with muscarin lengthens the refractory period without greatly affecting the bioelectric variation. ${ }^{2}$ From general considerations a complete coincidence is hardly to be expected. Although the existence of a certain normal bioelectric potential between protoplasm and medium, the so-called "physiological polarization," is apparently necessary for stimulation, it is known that a tissue may be rendered inexcitable by conditions that have little effect on this potential, e.g., the presence of anæsthetics, Mg salts, etc. It is probable that the normal physiological polarization is regained rapidly during the early part of the refractory period, as shown by the completion of the return variation of potential; this change apparently indicates the return of the altered

${ }^{x}$ Cf. Hering, "Theory of the Functions in Living Matter," Brain, XXII (I897), 232 (translation of article in Lotos, IX, Prague [r888]).

${ }^{2}$ Cf. Trendelenburg, loc. cit.; also the discussion in Adrian's paper, loc. cit. (I92 I). 
surface-film to its previous continuous and semipermeable state; yet the film may require some further structural or chemical modification before it is in a condition favorable for stimulation and transmission. The relative refractory period seems to correspond to the time during which those regulatory or restorative changes are proceeding in the newly re-formed film.

These general considerations receive support of an indirect kind from my recent experiments on transmission and recovery of transmissivity in passive iron wires immersed in nitric acid solution. ${ }^{x}$ In this inorganic model there is also a refractory or non-transmissive period immediately following the passage of an activationwave. During the progress of the chemical reaction following the activation of a passive wire in 60 per cent $\mathrm{HNO}_{3}$, the metal exhibits the chemical and electrical properties of ordinary or active iron, and is insusceptible to further activation. This initial period of activity may be compared with the absolute refractory period in the living tissue. Its onset is accompanied by a variation of potential, the active metal becoming negative (anodal) relatively to its previous condition by $\mathrm{Ca} .0 .7$ volt. After a brief interval of vigorous chemical reaction, lasting from one to two seconds, the metal reverts spontaneously to the passive state, the effervescence ceases, and the potential immediately becomes again positive. During the first minute or so after this automatic repassivation it is found impossible to reactivate the metal completely by local mechanical or chemical treatment; e.g., touching the wire with zinc produces a brief and partial activation which is transmitted for

I Jour. Gen. Physiol., III (1920), 107, I29. 
only a short distance; by degrees transmissivity returns, and in one-and-a-half or two minutes (at $20^{\circ}$ ) is as complete as before. In this case the return of passivity - with the potential characteristic of that state-depends on the re-deposition of a thin surface-film of oxidationproduct. This process is itself a rapid one, as shown by the rapid change of potential from negative to positive; but the metal is at first relatively resistant to alteration, and regains its former properties only by degrees, probably as a result of a progressive thinning, rearrangement of molecules, or other change in the film, accompanying the approach to the equilibrium condition. If we may regard the processes in the inorganic model as resembling in their general features those of the irritable living system, it would seem probable that in the latter the "absolute" refractory period represents the early phase in the local stimulation-process during which the alteration and breakdown of the protoplasmic surfacefilm are in progress; while the "relative" period is that during which the film is being rebuilt and reconstituted in the succeeding recovery-process. As the film returns toward the normal or equilibrium condition, the ability of the tissue to respond and transmit excitation also returns. It is interesting to note that the temperature-coefficient of the process of recovery in passive iron is high and apparently similar to that of living tissues $\left(\mathrm{Q}_{\mathrm{I} 0}=2-3\right)$.

PERMEABILITY-INCREASE AND STIMULATION

If during the local stimulation-process there is in fact a temporary breakdown or dissolution of the

I Ibid., p. I 26 
protoplasmic surface-film, a temporary increase of permeability to water-soluble diffusible substances should be associated with stimulation. The normal semipermeability of the living cell, implying impermeability to water-soluble substances of low molecular weight (neutral salts, sugars, and amino-acids), depends on the structural continuity of the plasma membrane; and when this continuity is interrupted in any way, the effect is equivalent to a loss of semi-permeability. Such an effect may be temporary and difficult to detect in those cases where the surface-film is rapidly re-formed; but in the more favorable instances we should expect to find direct evidence of increased permeability during stimulation, in addition to the indirect indications afforded by the bioelectric variation and the refractory period.

According to the present theory, the local electric effects upon which the transmission essential to stimulation depends are the result of local alterations of the cell surface, involving increased permeability. Conversely, therefore, we should expect permeabilityincreasing agents as a class to cause stimulation of irritable cells. The production of an "injury-current" by mechanical or other means shows that local interruption in the physical continuity of the cell surface forms a local circuit; and the formation of this circuit may be the means of starting a propagated disturbance or wave of excitation, in the same manner as a local interruption in the surface-film of the passive iron wirc starts a wave of activation. It is well known that the application of a cytolytic, i.e., permeability-increasing, substance to a living tissue produces a local electrical negativity, giving rise to an injury-current, and that this current is 
sufficiently strong to excite other irritable tissues. Hence the local production of such a current within the irritable element itself might be expected to cause stimulation in adjoining areas of the same element, an effect which would be repeated at the boundary of each area thus secondarily stimulated, and thus form the condition for a spread of excitation, in the manner already indicated.

There is, in fact, a large body of evidence indicating that any rapid local increase of permeability, however induced, acts as excitant to an irritable cell or element. Mechanical treatment, like pricking or sudden pressure, the sudden application of heat, and rapidly acting cytolytic agents all have a stimulating effect on muscle or nerve. It is interesting to note that many cells, not ordinarily classed as irritable, undergo rapid and spontaneous structural alteration or breakdown under conditions involving local increase of permeability. This is well seen in the effects following the puncture with capillary needles of the surface of red blood corpuscles and other cells; frequently a disintegration starts at the point of injury and rapidly leads to the breakdown of the entire cell. ${ }^{\mathrm{I}}$ In a red corpuscle thus treated the exit of hæmoglobin may be seen to begin simultaneously over the whole surface. ${ }^{2}$ It is clear that in such cases the surface-film undergoes a sudden and irreversible increase of permeability; this change is propagated over the whole surface, and apparently in many cases through-

${ }^{x}$ Cf. Kite, American Journal of Physiol., XXXII (1913), I46; Chambers, Science, XL (I914), 824; XLI (1915), 290; Oliver, Science, $\mathrm{XL}$ (IgI4), 645 .

${ }^{2}$ Cf. Chambers, Anatomical Record, X (Ig16), Igo. 
out the internal protoplasm as well. with the result that the cell breaks down. The rapid disintegrations characteristic of the "explosive" blood corpuscles of Crushuceu and of the vertebrate platelets, and the phenomena exhibited by other rapidly and irreversibly reacting cells like nematocysts and certain unicellular gland cells, appear to be examples of the same type of process. In all such cases the surface-film is stable only so long as its continuity is uninterrupted; interruption initiates a wave of disintegration which may involve the entire film-structure of the protoplasm.

In the cellular elements composing typical irritable tissues like muscle and nerve, in which the effects of stimulation are automatically reversed when stimulation ceases, the indications are that an essentially similar process of film-disintegration occurs during stimulation, but with the difference that a new surface-film is immediately re-formed. Whether the effects of stimulation are reversible or irreversible appears to depend on the ability of the protoplasmic system to re-form the structure necessary for stimulation. Under abnormal conditions, e.g., lack of oxygen, presence of depressant poisons, or disease, the rate of metabolic synthesis may be insufficient for complete restoration in the intervals of stimulation. and in such cases excessive stimulation may lead to the death of the cell. But, normally, recovery is rapid and complete in irritable elements of this class; as already pointed out, the indications are that the new film-structure is formed during the earlier part of the relative refractory period.

${ }^{x}$ Cf. W. B. IIardy, Journal of Physiology, XIII (1Sg) 2), 165; Tait, Quarterly Journal of Experimental Physiology, XII (1918), 42. 
The ability of living protoplasm to form fresh semipermeable surface-films at cut or injured surfaces has long been known, and with the introduction of the methods of micro-dissection this property has lately become the subject of renewed investigation. The rate and other features of the film-forming process vary widely in different forms of protoplasm, and also in the same form of protoplasm under different conditions; thus it is less active in cells that have been subjected to abnormal conditions than in normal and "healthy" cells. $^{2} \quad$ Apparently this process is of the same nature as the reconstructive change occurring at the surface of irritable elements after stimulation and occupying the first part of the relative refractory period. In many respects the refractory period resembles a brief period of fatigue; and it is well known that rapid recovery from fatigue, i.e., a complete restoration of the state preceding stimulation, requires that the supply of oxygen and the other environmental conditions should be normal. In the absence of these conditions excessive stimulation may lead rapidly to the physical disorganization and death of the protoplasm. Some of the effects of extreme fatigue (e.g., development of acid reaction, rapid onset of rigor) resemble those produced by cytolytic agents; and it is possible that certain metabolic products formed during activity have themselves a directly cytolytic

${ }^{x}$ Cf. Chambers, American Journal of Physiology, XLIII (igi 7), I, for a description of film-formation after injury in egg cells. Also Jour. Gen. Physiol., V (1922), I89.

${ }^{2}$ Seifriz (Annals of Botany, CXXXVIII [1921], 269) cites numerous observations on film-formation in protoplasm under various conditions; cf. also his article in Botanical Gazette, LXX (1920), 360. 
effect. Hence, their removal, independently of other conditions, is favorable to recovery. But the main factor in the reversal of the effects of stimulation, normal or abnormal, appears to be the synthetic or structureforming (formative) metabolism of the protoplasm. When the metabolic rate is rapid, recovery from stimulation or injury is prompt and complete, and vice versa. The difference between the recuperative and regenerative powers of young and old individuals in higher animals illustrates this condition.

\section{STIMULATING EFFECTS OF PERMEABILITY-INCREASING AGENTS}

Chemical or physical agents whose primary effect is to increase the permeability of the cell surface to watersoluble substances have as a class a strongly stimulating action on many irritable forms of protoplasm. The physiological effects produced by salts and combinations of salts in isotonic solution illustrate this very clearly. Thus pure solutions of neutral alkali salts, e.g., $\mathrm{NaCl}$, have in general a rapid permeability-increasing action on living cells; this effect, if unreversed, is equivalent to toxic, and is prevented by the addition of a small proportion of $\mathrm{CaCl}_{2}$ or similarly acting salt to the solution; the antagonistic or "anti-toxic" action of the latter salt is to be referred chiefly to this preventive influence, which is equivalent to protective or anticytolytic. Correspondingly, pure solutions of $\mathrm{Na}$ salts cause stimulation or activation in many cells; and this effect also is prevented by addition of calcium in proportions similar to those required to prevent increase of permeability. The twitching of vertebrate skeletal muscle 
in pure $\mathrm{NaCl}$ solutions has been well known since Sidney Ringer's time: ${ }^{\mathrm{I}}$ this solution also causes rhythmical stimulation in motor nerve, although with a somewhat prolonged latent period; ${ }^{2}$ in both cases the effect is prevented by the presence of a little $\mathrm{CaCl}_{2}$. The muscle cells of marine animals are also powerfully stimulated by pure isotonic $\mathrm{NaCl}$; an especially instructive case is furnished by the larva of the annelid Arenicola, in which the contraction in the pure $\mathrm{NaCl}$ solution is very energetic. The body-cells of this organism contain a yellow pigment which serves as an indicator of increase of permeability. In the pure $\mathrm{NaCl}$ solution the pigment diffuses into the surroundings simultaneously with the contraction; and under a wide range of conditions the permeability-increasing and the stimulating effects of different salt solutions have been found to run closely parallel. Thus both effects are prevented or diminished by the addition of $\mathrm{CaCl}_{2}$ or $\mathrm{MgCl}_{2}$ to the pure $\mathrm{Na}$-salt solution; and the addition of organic anæsthetics in the anæsthetizing proportions has a similar action. ${ }^{3}$ Similarly pure isotonic alkali salt solutions cause activation and increase of permeability in unfertilized starfish and sea-urchin eggs, and both effects are prevented by calcium salts or anæsthetics. ${ }^{4}$

In general, any condition that prevents or retards the increase of permeability normally produced by the pure salt solution prevents or diminishes stimulation. The anti-stimulating or narcotic action of compounds

$\therefore$ Cf. chap. viii.

2 Mathews, American Journal of Physiology, XI (I904), 455.

3 Cf. chaps. viii, ix.

${ }_{4}$ Cf. p. 166. 
like the organic anæsthetics or Mgr salts appears to be associated with a characteristic action on the protoplasmic surface-films; the latter are rendered more stable, i.e., protected against the permeability-increasing or disintegrative action of the pure salt solution; as we have seen, a definite antitoxic influence is exerted by these compounds when they are added in appropriate concentrations to the pure salt solution. It is probable that this stabilizing influence is responsible for the general narcotizing properties of such compounds, since any condition preventing alteration of the surface-films must by that very action prevent stimulation, which is dependent upon such alteration.

The activating effect of pure alkali-salt solutions upon the unfertilized eggs of marine animals is a phenomenon which has many important physiological affinities with stimulation, and is exhibited under similar conditions. If starfish eggs are placed in pure isotonic solution of $\mathrm{NaCl}$ (or similar salt) for five to ten minutes $\left(\right.$ at $20^{\circ}$ ) and are then returned to sea water, a considerable proportion form fertilization-membranes and cleave, or even develop to a free-swimming stage. ${ }^{3}$ But the same solution to which $\mathrm{CaCl}_{2}$ has been added (I mol. to 20 alkali salt) has little or no effect. ${ }^{2}$ 'The eggs of Arbacia are more resistant to this type of salt action, and are only slightly affected by $\mathrm{NaCl}$ solution; but pure isotonic solutions of more energetically acting alkali salts, especially the iodides and thiocyanates of $\mathrm{Na}$ and $\mathrm{K}$, form fertilization-membranes and initiate the activation-process in the same manner as other cytolytic

I American Journal of Physiology, XXVI (1910), 106.

2Ibid., XXVII (IgII), 289. 
agents; ${ }^{\mathrm{I}}$ in these eggs an after-treatment with hypertonic sea water is required to complete the activation. Solutions of these salts containing $\mathrm{CaCl}_{2}$ or $\mathrm{MgCl}_{2}$ are ineffective; pure isotonic solutions of $\mathrm{CaCl}_{2}$ and $\mathrm{MgCl}_{2}$ also fail to induce activation; apparently the initial effect of the activating agent must be to increase permeability, while the alkali earth salts have the reverse effect. This is probably the reason why when added to the pure alkali salt solution they counteract those effects (activation, stimulation, and toxic action) which are dependent on increase of permeability.

That the initial effect produced by the pure alkali salt solution is a permeability-increasing one is to be inferred from the general nature of the action of such solutions on living cells. The evidence is more direct in the case of pigmented eggs like those of Arbacia, in which the pigment visibly diffuses into the surrounding pure solution (of NaI, KCNS, etc.) after a few minutes' immersion. In the Ca-containing solution this effect is absent; the permeability-increasing and the activating effects are thus simultaneously prevented. A similar though less complete preventive effect is produced by various anæsthetizing compounds, especially higher alcohols; these when present in the anæsthetizing concentrations (i.e., those which arrest celldivision reversibly) retard or prevent the permeabilityincreasing and activating action of the pure solutions. ${ }^{2}$

Loeb's extensive researches on artificial partheno genesis have shown conclusively that permeability-

x Journal of Morphology, XXII (I9II), 695.

${ }^{2}$ Journal of Experimental Zoölogy, XVI (I9I4), 59I. 
increasing substances in general (equivalent to cytolytic with long-continued action), whatever their special chemical nature, have the same activating effect on seaurchin eggs. ${ }^{x}$ The substances used included numerous lipoid-solvent organic compounds, acids, bases, soaps. alkaloids, cytolytic glucosides (saponin, etc.), and foreign blood sera. A brief or superficial cytolytic action is thus regarded by Loeb as the initial or critical change in activation. This use of the term "cytolytic" seems, however, to be open to objection, since, as usually: employed, it implies an irreversible or destructive effect on the cell; whereas the primary or critical change produced in activation is apparently a brief temporary increase of surface-permeability resulting from a rapidly reversed breakdown of the protoplasmic surface layer. The connection between this effect and activation is undoubtedly highly indirect and complex; but the same may be said for the connection between the direct action of any stimulating agent on an irritable tissue and the succeeding response of the latter. What is significant is that in both cases the primary or initiatory change in the complex physiological sequence appears to consist in a temporary disruption or breakdown (an effect probably related to de-emulsification) of the protoplasmic surface layer. This change furnishes the releasing condition for those metabolic and other processes of which the characteristic vital "response" is the eventual and biologically important consequence or expression. Physical agents like ultra-violet radiation or heat $\left(30-40^{\circ}\right)$, which also cause cytolysis in unfertilized eggs

IArtificial Parthenogenesis and Fertilization, University' of Chicago Press (I9I3). 
and other cells, exhibit under appropriate conditions a similar activating influence. ${ }^{x}$

INCREASE OF PERMEABILITY DURING NORMAL STIMULATION, ACTIVATION, AND CELL-DIVISION

Direct proof of the increased permeability of rapidly responding tissues like vertebrate muscle or nerve during normal stimulation is difficult to obtain. In the case of muscle the results gained with the method of electrical conductivity ${ }^{2}$ are of uncertain value, since the change in the form of the tissue, the production of electrolytes (e.g., lactic acid) in the process itself, and the change in the distribution or quantity of the intercellular fluids (lymph), all affect the total conductivity; hence the observed alterations may depend on other factors than the changing permeability of the plasma membranes. Direct observation of the penetration of easily detectable compounds into the muscle cell has given better results; recently Mitchell and his associates ${ }^{3}$ have shown that rubidium and cæsium chlorides do not enter the muscle cells when the resting muscle is perfused with Ringer's solution containing these salts, but penetrate readily when the tissue is thrown into contraction by stimulating the nerve. In this case there seems to be an unequivocal demonstration of increased permeability to inorganic salts during stimulation. Embden

${ }^{3}$ For the activation of starfish eggs by heat cf. Delage, Arch. zool. expér. et gén., IX (I $90 \mathrm{I})$, Series III, 285 ; R. S. Lillie, Journal of Experimental Zoölogy, V (Ig08), 375; Biological Bulletin, XXVIII (I9I5), 260. For the action of ultra-violet rays cf. J. Loeb, Science, XL (I9I4), 680; R. S. Lillie, American Journal of Physiology, LX (1922), 57, 272. 302.

${ }^{2}$ Cf. McClendon, American Journal of Physiology, XXIX (IgI2), ${ }^{3}$ Mitchell, Wilson, and Stanton, Jour. Gen. Physiol., IV (I92I), I4I. 
finds that frogs' muscle gives off inorganic phosphate to the medium during contraction but not during rest, and he regards this fact as further evidence of an increase of permeability during stimulation. ${ }^{\mathrm{x}}$

In nerve evidence of increased permeability during stimulation is seen in a characteristic decrease in the electrical polarizability of the tissue. When a current from a battery is passed by non-polarizable electrodes through any living tissue, a counter electromotive force is immediately set up in the tissue, so that when the latter is connected with a galvanometer (preferably through a double key which simultaneously breaks the polarizing circuit and opens the circuit through the galvanometer) a temporary current is observed flowing in the reverse direction. This current, the polarization current, hats its source within the tissue at the regions of entrance and exit of the original or polarizing current. Apparently the characteristically high resistance of living cells and tissues to the electric current is largely or mainly a result of this polarization, since the resistance to rapidly: alternating currents (which cause little or no polarization) is found to be much less than to direct currents. 'The indications are that the semi-permeable membranes of the living cells are the chief seat of the polarization; when semi-permeability is lost, as at death, polarizability is greatly diminished or disappears. Some fifty years ago Grünhagen and Hermann observed that battery currents flowing through a nerve underwent an increase when the nerve was stimulated; and the most probable

I Embden, Berichte iiber d. ges. Physiol., II (1920), 159.

2Cf. Hermann, "Das galvanische Verhalten einer durchlosenen Nervenstrecke während der Erregung," Arch. ges. Physiol., VI (1\&72), 560; cf. also ibid., X (1875), 215. 
interpretation of this effect is that it is an expression of decreased polarizability. This view is confirmed by Bernstein's observation that the electrotonic currents of nerve, which are undoubtedly polarization currents, are also decreased during stimulation. ${ }^{\mathbf{I}}$ Any such decrease of polarizability, in a system partitioned by membranes, is an indication of increased permeability of the membranes to ions. Recently Ebbecke has again shown that the polarizability of nerve is decreased during stimulation; ${ }^{2}$ he has also found that the same is true of the epidermal cells of the human skin, and he has discovered various interesting parallels between the phenomena exhibited by nerve and by skin, respectively, during and after the passage of the electric current. ${ }^{3}$ The polarizability of the skin and its resistance to constant currents are greatly decreased by either mechanical or electrical stimulation, and this effect is independent of variations of vascularity or other extracellular conditions. The reactions to the constant current are also typical: opposite effects are produced at anode and cathode, as in the polar stimulation of irritable tissues, and the action of salts and anæsthetics on the skin is analogous to that observed with other irritable tissues and cells; e.g., conductivity is increased by solutions of $\mathrm{Na}$ and $\mathrm{K}$ salts and decreased by $\mathrm{Ca}$ salts and anæsthetics. ${ }^{4}$ These

× Bernstein, Arch. Anat. Physiol. (I866), p. 6r4; also Elektrobiologie, chap. vii, p. I30. Cf. also Gotch's article in Schäfer's textbook, II, 547 ff.

${ }^{2}$ Ebbecke, "Membranänderung und Nervenerregung," Arch. ges. Physiol., CXCV (1922), 555.

3 Ebbecke, Arch. ges. Physiol., CXCV (1922), 300, 324.

4 Ebbecke, ibid., CXC (I92I), 230; cf. pp. 247 ff. 
effects receive a consistent explanation on the theory of variations of permeability.

The implication that apparently inert cells like epidermal cells are irritable, in the same sense as muscle and nerve, may seem a strange one, but it is in harmony with the general conception of irritability as an elementary property of all forms of living matter. Waller has pointed out that the most certain "sign of life" in an apparently inert animal or plant tissue is the elicitation of an electric response ("blaze-current") on mechanical stimulation. ${ }^{I}$ The increased proliferative activity of the epidermal cells of the skin after hard mechanical usage is well known. Ebbecke's experiments show that such treatment increases the electrical conductivity; in other words, increases permeability and decreases polarizability, as in other cases of stimulation. The increased growth is the expression of this stimulation.

Recent observations by Crozier $^{2}$ have also a bearing on the present problem. He finds that electrical stimulation causes a well-marked increase in the permeability of the mantle-cells of nudibranchs to acids; in these cells the degree of permeability can be measured by the time required to change the color of an intracellular pigment, which acts as a natural indicator. Mg salts and anæsthetics (in appropriate concentrations) were found to decrease permeability, as in the cases already cited. ${ }^{3}$ Mechanical traction causes an increase of permeability, which within certain limits is reversible. These observations throw much light on the general

${ }^{s}$ Waller, Signs of Life.

${ }^{2}$ Jour. Gen. Physiol., IV (1922), 723.

${ }^{3}$ Cf. chap. viii. 
conditions of mechanical stimulation; related observations are those of Carlson, who observed that increasing the mechanical tension of the heart-ganglion of Limulus increased the rate of nervous discharge; $;$ and in frog's muscle it has been found that stretching causes an increase in the production of $\mathrm{CO}_{2}$ and lactic acid. ${ }^{2}$ Increase of permeability is probably a factor in both of these effects.

The effects of mechanical, electrical, thermal, and other changes of physical condition on indifferent or unspecialized cells of various kind (blood corpuscles, egg-cells, epithelial cells, etc.) all have a bearing on the question of the relation of permeability-change to stimulation. The constant current causes polar disintegration in many cells, an effect analogous to polar stimulation; similarly it causes polar secretion in cutaneous and mucous gland cells. ${ }^{3}$ The laking of blood corpuscles by induction shocks has also evident analogies to electrical stimulation.

There is abundant evidence of an increase in the permeability of the surface layer of many egg cells during the early stages of normal fertilization. In some cases the surface protoplasm undergoes extensive alteration and there results a visible loss or secretion of material to the exterior (Nereis, lamprey, frog). ${ }^{4}$ In the sea-urchin

${ }^{x}$ Carlson, American Journal of Physiology, XVIII (1907), I49.

${ }^{2}$ Eddy and Downs, A merican Journal of Physiology, LVI (1921), I88.

${ }^{3}$ Loeb, Arch. ges. Physiol., LXV (1896), 308.

${ }^{4}$ Cf., for Nereis, F. R. Lillie, Journal of Experimental Zoölogy, XII (I9I2), 4I4; for the lamprey, Bataillon, Arch. de zool. expér. et gén., VI (1910), Series 5, I28; for frog, Backmann and Runnström, Arch. ges. Physiol., CXLIV (1912), 287. 
egg the space between the fertilization-membranc and the egg surface contains a colloidal substance which is apparently separated from the egg at fertilization. ${ }^{2}$ Lyon has also observed an increased loss of catalase at this time. ${ }^{2}$ McClendon and Gray have shown that a significant increase in electrical conductivity occurs in the sea-urchin egg immediately after fertilization ${ }^{3}$ Increase in the rate of entrance of dyes, and apparently also of toxic substances, has also been observed. ${ }^{4}$ In the Arbacia egg the rate of exchange of water in hypertonic or hypotonic media is increased several times as a result of fertilization. ${ }^{5}$ A change in the protoplasmic surface layer, associated with increased permeability to water and water-borne substances, thus appears to be a very general accompaniment of both normal and artificial activation. These facts, taken as a whole, suggest that the first stage of the activation-process consists in a breakdown, followed immediately by a re-formation, of the external protoplasmic layer or plasma membrane.

Recent observations by Just ${ }^{6}$ have emphasized still more fully the resemblance between the primary or surface change in the activation of egg cells and in the stimulation of irritable tissues. In the large egg of the sand-dollar, Echinarachinius (ca. $140 \mu$ in diameter).

${ }^{5}$ Cf. Loeb, Parthenogenesis and Fertilization, chap. xx, [1. 20 \%.

${ }^{2}$ Lyon, American Journal of Physiology, XXV (1909), 199.

3 MIclendon, American Jourmal of Physiology, XXVII (1910), 240, J. Gray, Jour. Mar. Biol. Assoc., X (1913), 50.

$4 \mathrm{McClendon,} \mathrm{loc.} \mathrm{cit.;} \mathrm{Lyon} \mathrm{and} \mathrm{Shackell,} \mathrm{Science,} \mathrm{Xxxul} \mathrm{(1910)}$ 249; Harvey, Science, XXXII (1910), 565.

5 American Journal of Plyysiology, XL (1916), 249.

${ }^{6} \mathrm{Just}$, ibid., LX (1922), 516. 
the first visible effect of insemination is an alteration of the egg surface, beginning at the point of entrance of the spermatozoön. A liquefactive or secretory change occurs in the protoplasmic surface layer, in consequence of which a thin surface-film is separated to form the fertilization membrane; this process of separation is not simultaneous at all points on the surface, but progresses in a wavelike manner from the point of sperm-entry to the opposite pole, which it reaches about 20 seconds later $\left(\right.$ at $20^{\circ}$ ). During the propagation of this disturbance over the egg surface, the latter is altered in such a manner that the plasma membrane loses temporarily its normal tenacity and coherence; this effect is readily demonstrated by placing the eggs at this time in dilute sea water ( 60 vols. fresh plus 40 sea water) in which they undergo immediate and rapid disintegration. The breakdown of the surface layer can be seen to begin at the region where the fertilization membrane is beginning its separation. This period of instability lasts only for the brief period (about one minute) during which the "cortical reaction" is traveling over the egg surface; within about a minute after insemination the original resistance to dilute sea water has returned, showing a restitution of the normal coherent surface layer. Thus a characteristic surface-change, apparently accompanied by a local disintegration or disorganization of the plasma membrane, constitutes the first reaction of this egg to fertilization; this change is propagated as a wave over the cell surface and is followed by a reconstructive process restoring the original condition. As we have already seen, there are indications that a propagated surface-change of a similar kind, only with different time- 
relations and a different velocity of propagation, accompanies stimulation in irritable cells and nerve fibers.

During the formation of the cleavage-furrow in celldivision a similar reversible change in the physical consistency and coherence of the cell surface occurs in echinoderm eggs; ; and there are many indications that the same kind of change is of general occurrence in dividing cells. The case of cell-division is of special interest, since a rhythm of chemical change and of susceptibility to physical and chemical injury is also associated with the rhythm of the cleavage-process. Lyon's experiments indicate that at the time when the cleavage-furrow is forming in the Arbacia egg, the rate of evolution of $\mathrm{CO}_{2}$ is several times greater than in the intervals between cleavage; ${ }^{2}$ at this time the egg is also most susceptible to injury by heat, ultra-violet radiation, deprivation of oxygen, and poisons ( $\mathrm{KCN}$, acids, and organic compounds). The time-relations of the accompanying change in the plasma membrane can be followed readily and accurately by transferring successive portions of a single lot of recently fertilized A rbacia eggs (in which the cleavage-process is very regular and occurs simultaneously in all eggs) from normal sea water to clilute sea water ( 50 to 60 volumes fresh water in 100 of the mixture) at regular intervals before, during, and after the formation of the cleavage-furrow: ${ }^{3}$ Eggs which are thus treated some time before cleavage swell osmotically but without undergoing evident increase of permeability

${ }^{x}$ R. S. Lillie, Journal of Experimental Zoöl., XXI (1916), 3t9; Herlant, Comp. rend. soc. biol., LXXXI (1918), 151; Just, Americun Journal of Physiology, LXI (1922), 505 .

${ }^{2}$ Lyon, loc. cit.

${ }^{3}$ R. S. Lillie, loc. cit. 
or losing the power of development on return to sea water; at about the time when the furrow begins to form, there is a marked and rapid decline of extensibility and coherence in the plasma membrane, and the eggs show rapid loss of pigment and cytolysis when transferred to the dilute sea water. Eggs brought into dilute sea water a few minutes after the furrow is complete are found to have recovered the original resistance, and swell without cytolysis. These experiments show clearly that accompanying the division of the cell body there is a reversible change in the properties of the plasma membrane, this change involving both loss of coherence and increase of permeability. That the permeability as well as the physical tenacity of the membrane is altered is best shown by studying the behavior of the eggs in concentrated instead of dilute sea water; during the formation of the furrow the abstraction of water and shrinkage are distinctly less rapid and complete than before or after cleavage, a difference indicating a partial loss of semi-permeability at this time. Other indications of increase of permeability during cleavage have been noted by various observers (Harvey, Just, Lyon).

According to the present theory variations of electrical surface-potential should accompany these changes of permeability; and observations made at Woods Hole in 1904 by Miss Hyde, ${ }^{\mathrm{T}}$ using fish eggs, indicate that during the formation of the cleavage-furrow the blastodisk area becomes increasingly negative relatively to the general surface of the egg. Experiments in this field are, however, few in number as yet; and it would be desirable to repeat and extend these observations, using

×. H. Hyde, loc. cit. 
the thermionic amplifier to enhance the minute effects obtainable from single eggs, and the string galvanometer as the recording instrument. It should be noted, however, that Miss Hyde's observations are in conformity with those of other investigators who have found rapidly growing regions of plants and animals -i.e., those where cell-division is in active progress-to be electrically negative to more slowly growing regions. ${ }^{\mathrm{x}}$

Evidence that reversible variations of permeability are associated with such processes as fertilization and celldivision may seem to have a somewhat indirect bearing on the problem of the conditions of stimulation in typical irritable tissues like muscle and nerve. Yet all of these vital processes are alike in being subject to initiation or control by environmental conditions or events; in other words, they all illustrate the characteristic "irritability" of living matter. The fundamental conditions determining and controlling the metabolic reactions which furnish the energy for vital processes are in all probability everywhere the same. Hence the above-cited facts indicating that changes of permeability are regular accompaniments of fertilization and cell-division are confirmatory evidence for the view that such changes play an essential part in other manifestations of irritability. In all forms of protoplasm the essential metabolic reactions occur under the control of the film-partitioned or emulsion-like structure of the living system; it is therefore to be expected that they will vary in their rate and character

r Cf. Hermann and Müller-Hettlingen, Arch. gis. Physis, XXXI (I883), г93; A. P. Mathews, American Jourmal of I'syriolasy, III (1903), 294; C. M. Child, Biologicul Bulletin, XLI (1021), 90; E. J. Lund, Journal of Experimenlal Zoölogy, XXXVI (1922), 47;; 11; man and Bellamy, Biological Bullctin, XLIII (1922), 313. 


\section{PROTOPLASMIC ACTION AND NERVOUS ACTION}

with alterations in this structure. In particular the foregoing evidence indicates that the temporary breakdown of the semi-permeable surface-lamella or limiting layer of the protoplasmic emulsion influences profoundly the chemical and other processes occurring in the cell interior; apparently the effects of this surface-change are transmitted throughout the whole mass of protoplasm, and the physiological activities of the cell are changed correspondingly.

It has already been mentioned that experiments of a kind closely analogous to those described for unfertilized egg cells may be performed with irritable tissues like muscle and nerve, both of which when immersed in pure isotonic solutions of neutral sodium salts undergo rhythmical or other stimulation, which may be checked by calcium salts or anæsthetics; and the above-cited experiments with Arenicola larvæ afford other and more direct evidence that stimulation and permeabilityincrease are closely associated. Höber's experiments on the influence of anæsthetics in checking the development of the negative electrical variation produced in muscle by application of $\mathrm{KCl}$ solution furnish evidence of a similar kind. The effects of neutral salts may thus be more or less completely antagonized by anæsthetics as well as by the alkali earth cations. In all of these cases prevention of permeability-increasing action runs parallel with prevention of stimulation or of the normal manifestations of stimulation. ${ }^{\mathrm{I}}$

Conversely any strongly cytolytic action has a stimulating effect. The larvæ of Arenicola contract strongly in solutions of cytolytic agents like chloroform; this effect

${ }^{x}$ Cf. chaps viii, ix. 
is irreversible and is associated with a marked increase of permeability and rapid death. Phenomens of a similar kind are seen in vertebrate skeletal muscle. The permanent or irreversible shortening or "contrao ture" of frogs' muscle in solutions containing cytolytic substances, e.g., saturated solution of chloroform in Ringer's solution, is well known; this contraction is associated with a large production of lactic acid, and apart from its irreversible or "rigor" character bears many resemblances to normal contraction. A similar contraction accompanies the onset of heat-rigor and other forms of death-rigor, and in all such cases the structure of the cells is profoundly altered, the permeability undergoing marked increase while the fibrils lose their tensile strength and elasticity. These changes have a close general resemblance to those already described as accompanying the accelerated rhythmical activityindicating excessive stimulation-of the ctenophore swimming plate in pure $\mathrm{Na}$ salt solutions.

\section{SENSITIZATION AND RELATED EFFECTS}

The foregoing contraction-producing action of cytolytic substances on frog's muscle may be made to resemble more closely the phenomena of normal stimulation by first "sensitizing" the muscle by immersing it for a few minutes in a pure isotonic solution of a neutral Nit salt $\left(\mathrm{NaCl}, \mathrm{NaBr}, \mathrm{NaI}, \mathrm{NaNO}_{3}\right.$, etc.). The fresh isoluted gastrocnemius (normal or curarized), immersed in Ringer's solution and arranged so as to write upon at smoked drum, is transferred for four or five minutes to the pure solution of the $\mathrm{Na}$ salt, from which it is brought directly into the solution containing the cytolytic 
substance. The contraction then resulting is much more rapid and vigorous than in the control muscle which is brought into the same solution directly from Ringer; the degree of permanent shortening is also greater, as is also the degree of coagulation of the muscle protoplasm, as shown by the whitening or opacity produced. The action of contraction-producing salt solutions like $\mathrm{KCl}$, $\mathrm{Na}$ tartrate, sulphate, and citrate is similarly intensified by a previous bath of the kind above; also the rapidity of onset of heat-rigor, with the associated contraction, when the muscle is dipped in warm Ringer's solution $\left(38^{\prime}-40^{\circ}\right)$. These sensitization-effects have been demonstrated with the following cytolytic substances: chloroform, cytolytic glucosides (saponin, digitalin, aconitin, and agaricin), tetanus toxin, rattlesnake venom, foreign blood sera (horse, dog), and soaps. The degree of the stimulation following the introduction of the saltsensitized muscle into the solution, as indicated by the rate and degree of the contraction, is in general proportional to the intensity of the cytolytic action (as shown by varying the concentration and nature of the cytolytic substances). ${ }^{x}$

Since in all such experiments the contraction follows immediately (within a second or less) after placing the muscle in the stimulating solution, there seems to be no doubt that the initiatory effect consists in an alteration of the external surface layer of the muscle cells. Apparently, when the normal muscle is exposed to the pure salt solution, the cell surface is rendered more susceptible to alteration by external chemical agents, and the susceptibility to chemical stimulation of the kind above

${ }^{1}$ American Journal of Physiology, XXVIII (I9II), 197; cf. p. 214. 
is correspondingly increased. The relative insusceptibility of the normal muscle depends on the presence of $\mathrm{Ca}$ salts in the external medium; if, instead of a pure solution of the sensitizing salt, one containing $\mathrm{CaCl}_{2}$ (I $\mathrm{mol} \mathrm{CaCl}_{2}$ to $20 \mathrm{Na}$ salt) is used, no such effects are obtained. ${ }^{\mathbf{T}}$ The sensitizing action is thus subject to typical salt-antagonism, like so many other biological processes, especially those involving alteration of the protoplasmic surface layers. Muscles which have been rendered hypersensitive by exposure to the pure salt solution, rapidly recover their normal properties on return to Ringer's solution. The inverse type of effect. decrease of susceptibility to chemical stimulation, may be induced by a similar exposure to isotonic solutions of $\mathrm{CaCl}_{2}, \mathrm{MgCl}_{2}$ or similar salts. Such desensitizing effects are closely related to those classed under narcosis, depression, or anæsthesia, and are also reversible in Ringer's solution.

A related type of salt sensitization, produced by: isotonic solutions of $\mathrm{Na}$ salts whose anions precipitate calcium (or remove $\mathrm{Ca}$ ions from solution), was described by Loeb in I90г. ${ }^{2}$ Muscles dipped for a few minutes in solutions of $\mathrm{Na}$ sulphate, tartrate, citrate, or similar salt, and then brought into the air (or other foreign medium, e.g., oil), exhibit vigorous tetanic contractions, which cease or are diminished on return to the salt solution. Apparently this reaction depends on an altered contact-sensibility, due to some modification of the cell surface. It illustrates a type of effect which appears to be widely prevalent in irritable elements.

"Unpublished observations in the Biological Laboratory" of Clark University.

${ }^{2}$ J. Loeb, American Journal of Physiology, V (Ig01), 362. 
Nerve is affected similarly; and the characteristic pharmacological effects produced by this group of salts, e.g., their cathartic action (which apparently depends upon a heightening of contact-irritability in the intestinal tract), are probably referable to conditions of a similar kind. There is much evidence that many forms of pharmacological action are due to changes in the physical consistency, permeability, chemical alterability, or other properties of the protoplasmic surface-films.

It is important to note the relation of sensitizations of the class described-which consist in a general heightening of irritability toward non-specific chemical or other stimulating conditions-to the class of specific sensitizations, of which anaphylaxis is the most striking example. The general features of this phenomenon are well known. During the early stages of the process of immunization, following the introduction of a foreign protein into the circulation, the cells of the mammalian organism become highly sensitive to the introduction of further protein of the same kind, and in certain animals, notably the guinea-pig, the most conspicuous effect of the second injection is seen in the smooth muscle cells, especially those of the respiratory tract; these contract firmly and persistently and occlude the bronchioles, with death by asphyxiation as a consequence. This contraction-producing effect is almost certainly dependent on a specific antigen-anti-body reaction occurring in the surface layer of the muscle cells. The promptitude with which it follows injection of even a small quantity of the foreign protein indicates this, since the latent period seems insufficient for penetration into the cell interior; such a conclusion receives further support 
from the facts of passive sensitization, in which the sensitized condition is produced in a normal guineat-pig by injection of blood from another animal which has already been immunized to the protein in question. Passive sensitization can also be produced in silro by bathing strips of uterus with serum from an immunized animal. The most probable interpretation of this phenomenon is that the circulating anti-body is adsorbed or fixed by contact with the smooth muscle cells, thus becoming a constituent of the protoplasmic surface layer. When the antigen (the original protein used for immunization) is introduced, it reacts with the adsorbed anti-body, and in so doing alters the structure or consistency or permeability of the surface layer (very much as a specific cytolysin would do) in a manner corresponding to strong stimulation. Contraction then results; and since the antigen-anti-body reaction is an irreversible one, the muscle cells remain firmly and persistently contracted, with results fatal to the animal Dale has brought forward evidence that the specific chemical interaction underlying anaphylactic shock is identical with the precipitin reaction, the difference being that the reaction occurs within the cell instead of in the blood stream. ${ }^{x}$ If this is the case, it is easy to understand why powerful stimulating effects should result from precipitation of proteins within the protoplasmic surface-film, since such a process must alter the structure and permeability of this layer and hence act as a stimulating condition in the sime manner as any other cytolytic change would do.

${ }^{x}$ Dale, Croonian Lecture, Proceedings of the Royal Society, B, XCI (1920), I 26 . 
According to this conception the anaphylactic reaction of smooth muscle cells becomes an example of specific chemical stimulation, dependent on the presence of specific substances (presumably protein) in the surface layer of the reacting cell; these substances react with substances of corresponding or complementary configuration in the environment, and hence furnish the conditions for a highly selective and specific type of response. Presumably other forms of specific chemical sensitivity are also to be referred to the presence of specific chemical compounds in the surface-films of the reacting cells. Such a conception renders clearer the general nature of the relation between the special chemical sensitivity exhibited by a particular species of cell and its specific chemical organization. Any change in the chemical constitution or physical state of the plasma membrane must influence irritability and hence the other properties or activities controlled by this region of the cell.

\section{OTHER INDICATIONS OF THE CONNECTION BETWEEN}

INCREASE OF PERMEABILITY AND STIMULATION

Certain instances of stimulation-effects which are associated with an evident increase of surfacepermeability have already been cited. Perhaps the clearest instance of a direct dependence of a normal functional response upon a sudden increase of permeability is seen in the osmotic motor mechanisms of plants, such as the Venus' flytrap and the sensitive plant. In the latter plant, Mimosa pudica, the leaves are kept in the normal expanded and upright position by turgid or water-distended masses of parenchyma cells (pulvini) 
at the base of each leaflet and petiole. 'This turgor, as in other herbaceous tissues, is maintained by the osmotic pressure of the cell-contents; this pressure, acting against the semi-permeable plasma membranes, causes the entrance of water from the intercellular spaces and distends the cells until the pressure is equilibrated by the elastic tension of the stretched cellulose cell walls. Evidently the continued maintenance of this condition depends on the preservation of semi-permeability. On stimulation there is a sudden loss of turgor, accompanied by exit of water and dissolved substances from the cells; the stretched cell walls of the pulvini contract, the leaves fall, and the leaflets fold together. Apparently stimulation renders the plasma membrane suddenly permeable to the osmotically active intracellular sub)stances which maintain turgor. This effect is reversible, and under normal conditions turgor is gradually regained. The leaves of the Venus' flytrap and the sensitive contractile stamens of the Cynarea show a behavior essentially similar to that of Mimosa. Temporary loss of semi-permeability due to mechanical stimulation seems to be a not uncommon phenomenon in plant cells; Pfeffer cites the "stimulatory plasmolysis" of diatoms and other plant cells as cases of this kind, although he apparently hesitates to apply this explanation to the pulvinus of Mimosa.

The general rules of stimulation apply to these osmotic motor mechanisms of plants, in the same manner as to the excitation-processes of animal tissues. Electrical stimulation, summation, and anesthesia occur under conditions similar to those described above,

x Physiology of Plants, English translation, III, 75. 


\section{PROTOPLASMIC ACTION AND NERVOUS ACTION}

although the quantitative relations are different; hitherto these relations have been less completely investigated in plants than in animals. Transmission of excitation in plants resembles that of slowly conducting animal tissues, ${ }^{\mathrm{I}}$ and the excitation-process is accompanied by a negative bioelectric variation. A prolonged refractory period succeeds the motor response in Mimosa, and this condition also appears to be general in plants. The presence of a high degree of turgor in plant cells renders the evidence of a temporary loss of semi-permeability during excitation in many respects more definite and complete than in the case of animal tissues; but in other respects the fundamental processes underlying stimulation appear to be of the same kind in both groups of organisms.

In the higher animals the phenomena accompanying the secretion of gland cells, especially those under nervous control, show many resemblances to those just described for motile plant tissues. There is the same loss of water and dissolved material from the cell, the same electromotor variation, and the same gradual recovery. The variations in the permeability of the mammalian kidney cells under the influence of fear, excitement, or other abnormal emotional or nervous conditions also suggest that in these cells stimulation is associated with increased permeability; similar evidence is furnished by sweat glands. The secretory phenomena accompanying fertilization in many egg cells have already been mentioned.

It might be objected that evidence drawn from the observation of special tissues whose normal function consists in the separation of dissolved substances, either formed within the cells or collected from the surround-

${ }^{x}$ Cf. Bose, Proceedings of the Royal Society, B, XCIII (1922), 153 . 
ings, is scarcely applicable to the case of irritable elements in general. But such facts at least show plainly that stimulation is often associated with increased permeability or other evidence of temporary structural breakdown; and the fact that the conditions under which stimulation occurs in other irritable living systems, and also its most general manifestations such as the bioelectric variations, are of the same kind in the turgor-motor cells of plants and in gland cells as in muscle and nerve points clearly to the existence of some fundamental physico-chemical condition common to all such promptly reacting irritable systems. If, as the present theory holds, this condition consists in the temporary alteration or breakdown of the film-structure which surrounds and pervades all protoplasmic systems, the resemblances are intelligible; while in any case the differences are to be attributed to special peculiarities of structure and organization.

The phenomena of luminescence in animals furnish additional evidence that stimulation is associated with the temporary breakdown or removal of semi-permeable partitions within the living system. The production of light in irritable luminescent organisms like Noctilual may be regarded as an index of stimulation in very much the same sense as the bioelectric currents are such an index; and probably both phenomena are conditioned by structural changes of a similar kind. The investigations of Dubois and Harvey indicate that in many if not all luminescent animals light-production depends on the union of the two photogenic components, luciferin and luciferase, in the presence of oxygen. ${ }^{2}$ In a lumi-

'Cf. Harvey's recent book, The Nature of Animal Light (Philadelphia, 1920). 
nescent cell which responds to stimulation by a flash of light, all three substances are apparently present and available, but during the resting state they are prevented from uniting by the presence of protoplasmic films or partitions; when as a result of stimulation these partitions are temporarily broken down, chemical union and light-production result. Further analysis of the conditions of luminescence in irritable cells will no doubt throw much light upon the general nature of stimulation processes.

Harvey $^{\mathrm{I}}$ has recently made some simple and striking experiments on plant tissues, giving further indication that in living cells under normal conditions chemical reactions are frequently prevented or restricted by the presence of protoplasmic partitions or membranes impermeable to the interacting substances. The oxidase reactions which cause the browning of potato, apple, or similar tissues are examples. If a potato is cut in the presence of oxygen, the browning occurs only at the cut surface; even in pure oxygen under high pressure, the interior tissue remains unchanged. This absence of effect cannot be referred to an impermeability to oxygen, since all of the physiological and chemical evidence indicates that living protoplasm is freely penetrated by this gas. The oxidase and the chromogen are in some way prevented from uniting while the tissue is living. If, however, it is exposed for a few minutes to chloroform vapor, the browning extends rapidly throughout the whole mass. Apparently the effect of the chloroform is to break down the protoplasmic partitions which normally prevent free union of the compounds. Destruc-

${ }^{x}$ E. N. Harvey, Jour. Gen. Physiol., V (1922), 215. 
tion of semi-permeability is a universal effect of proisoning with chloroform or similar substances; this effect is seen in the wilting of turgid plant tissues, increase of electrical conductivity, or diffusion of substances (e.g... coloring materials) from the cells. The leares of the common false indigo plant, which blacken on death, also afford a striking demonstration. ${ }^{\mathrm{I}}$ If the leaves are poisoned with chloroform in the absence of oxygen (e.g., in a gais chamber with hydrogen), they remain green; if they are then exposed to air they blacken immediately. When living, intact leaves are exposed to oxygen at 100 atmospheres, no blackening results. On the other hand, mechanical injury, natural death, or poisoning all produce this effect at air tension. The essential condition for the reaction is apparently the destruction of diffusion-preventing partitions which during life keep the interacting substances apart. According to Chiari, ${ }^{2}$ autolysis of animal tissues is similarly hastened by ether or chloroform. It thus seems probable that in many if not all cells the external layer of protoplasm (plasma membrane) is not the only semi-permeable structure present, but that it is continuous with a system of similarly constituted films pervading the protoplasmic system and determining the spatial distribution of the water-soluble cell-constituents. ${ }^{3}$

If a temporary breakdown of film-structure cam determine chemical effects of this kind, the possibility presents itself that in cells of a different type of orginization, e.g., muscle cells, other chemical reactions, including

${ }^{s}$ Cf. Harvey, loc. cit.

${ }^{2}$ Chiari, Arch. exper. Path. u. Pharmakol., LX (1909), $25^{6}$.

${ }_{3}$ Compare Hofmeister's Chemische Organisation der Zalle. 
those yielding the energy for contraction, may be under similar kind of control. In these cells the chief reactions following stimulation probably occur at the surface of the contractile fibrils, and apparently certain reactionproducts, e.g., lactic acid, are directly concerned in the resulting contraction. ${ }^{I}$ We may assume that the temporary breakdown of the interfacial film (between fibril and sacroplasm) will have a double effect: (I) permit access of diffusible substances (possibly of the lactic acid) to the interior of the fibril; and (2) form the condition of a change of surface-tension, in the same general manner as in the $\mathrm{Hg}-\mathrm{H}_{2} \mathrm{O}_{2}$ system. Under these conditions contractile effects (essentially of an electrocapillary kind) would result. Hence the consideration of the relation of film-structure to the chemical reactions of protoplasm has an obvious bearing on the problem of the conditions of contractility in muscle and other contractile tissues.

I For a discussion of the part played by acids in the contractile mechanism cf. the recent review of A. V. Hill, Physiological Reviews, II (1922), 3 10. 


\section{CHAPTER XV}

THE PHYSICO-CHEMICAL B.ASIS OF TRANSMISSION IN NERVE AND OTHER PROTOPL.IS.IIC SYSTEMS

The view that the transmission of the excitationstate from the active region of an irritable protoplasmic element to the adjacent resting region is the result of secondary electric stimulation by the local bioclectric current between the two areas is one which is supported by general theoretical considerations and by a varicty of direct and indirect evidence. In a general sense there is nothing novel about this hypothesis, which, like most scientific conceptions, has had its historical background and development; it was expressed tentatively by I) u Bois-Reymond ${ }^{\mathrm{x}}$ and in a more definite form by Hermann; more recently Kühne, Cremer, Gotch, Keith Lucas, and others have supported it on various grounds. ${ }^{3}$ 'The absence in nerve of any observable accompaniment of the local excitation process, other than the electric variation, which could conceivably serve as a stimulus to the resting region adjoining the active area, is in

IGesammelte Abhandlungen zur allgemeincn. Iuskel und Xeronphysik, II, p. 698; cf. p. 733 .

${ }^{2}$ See especially the clear statement by Hermann in his Handbuch, II, 194, cited in Cremer's comprehensive article on nerve physiology in Nagel's Handbuch der Physiologie, IV, 2d half (1909), 929.

3 Kühne, Croonian Lecture, Proccedings of the Royal Socidy, XIII (1888), 446; Cremer, loc. cit.; Gotch, article on nerve in Schafer's tertbook, cf. pp. 458, 557 ff.; Kcith Lucas, Journal of P'hysiology, XXXIX (x909), 207. 
itself a strong argument in its favor. It is surprising that until recently it has received relatively little serious consideration from physiologists, most of whom have been apparently content to regard the bioelectric phenomena as inessential by-products of protoplasmic action.

The importance of the electrical factor in the phenomena of protoplasmic transmission is, however, clearly recognized in the "core-conductor" (Kernleiter) theory or theories of nervous action. ${ }^{I}$ This conception has as its basis the presence of characteristic polarization effects in a nerve through which a current is led (by non-polarizable electrodes); these effects closely resemble those exhibited by a system consisting of a simple metallic wire surrounded by a sheath or layer of electrolyte solution. The resemblance is so detailed, as regards the distribution, rate of development, and subsidence of the polarization potentials, that there can be little doubt of the essential identity of the physical conditions underlying these phenomena in the two systems. In nerve the surface of the axone has usually been regarded as the chief seat of the polarization, and Hermann especially has called attention to the intimate relations existing between polarization and stimulation. Neither he nor his successors, however, could explain satisfactorily, on the basis of the phenomena shown by simple polarization models of this type, the characteristic wavelike transmission of the electrical variation in the excited nerve. Apparently the presence of special physiological factors must be assumed, whose effects are superposed on those of the purely physical factors. This point of

${ }^{x}$ Cf. Cremer's larticle, op. cit., p. 904, for historical account and discussion. 
view has more recently been emphasized by. Cremer in an important series of studies on the "Kernleiter" theory, published at intervals since I 899 . He assumes in nerve, in addition to the physical polarization which this tissue exhibits in common with artificial systems of the coreconductor type, the presence of a special "physiological polarization,"x by which term he means some active process (in the nature of a response or reaction) exhibited at the regions of entrance and exit of the current; this process he conceives as based on a chemical change of some kind, which secondarily may alter polirization and hence serve as the source of a current. In this manner the polarization effect may be renewed at succesive areas of a nerve, and transmission to an indefinite distance becomes possible. ${ }^{2}$

In the foregoing form the Kernleiter theory requires only slight modification in order to make it entirely. consistent with the present form of the "membrane" theory. Both theories agree that a change of polarization is the critical or primary event in the local stimulation process. Evidently if the polarization is confined to the surface of the protoplasmic element (as also of the metal in a core-conductor), this critical change is a surface change. According to the membrane theory, the variation of polarization in stimulation is the result of a sudden change in those features of structure, composition, or permeability which determine the normal electromotor properties of the protoplasmic surface-film or plasma membrane. Such a change may result from

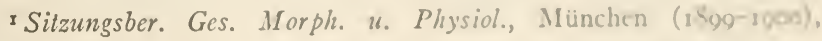
Hefte I and 2. $930 \mathrm{ff}$.

${ }^{2}$ See Cremer's exposition in his article in Nagel's I/andburh, pp. 
a chemical change in the substance of the membrane; the theory further assumes that this change is of such a kind as to be readily induced by the passage of a current.

The analogy between this hypothetical type of process and the phenomenon known as "local action" at metallic surfaces is a close one, to which I have recently called attention. ${ }^{\mathrm{I}}$ An example is the spread of corrosion in metals like iron in contact with electrolyte solutions; this spread is the result of local chemical action under the influence of local electrical circuits between the altered and the unaltered areas of the metal. The possibility is thus suggested that in the irritable protoplasmic element the primary change in electrical excitation is also of the nature of an electrolysis. Through the chemical change thus induced the properties of the surface-film are altered in such a manner as to render it "negative" to unaltered areas; a local circuit then arises at the boundary between altered and unaltered areas and causes electrolysis in the latter, in the same manner as the original current; and by a repetition of this effect the chemical and electromotor change spreads. Evidently a wavelike transmission without decrement is theoretically possible under these conditions. The chief requirement is the presence of a uniform and chemically unstable film forming the boundary layer of the irritable element.

Conditions of essentially this kind are in fact realized in the passive iron wire in nitric acid solution; and in this simple inorganic system the phenomena of activation and transmission exhibit a surprisingly detailed resem-

$x$ "Electrolytic Local Action as the Basis of Propagation of the Excitation-Wave," American Journal of Physiology, XLI (I9I6), I 26. 
blance to those observed in nerve and other conclur timg protoplasmic systems. The possibility of tramsmisions of the general type above is thus demonstrated. and the problem becomes chiefly one of determining the sputial nature of the conditions present in the living system. It is obvious that polarization effects are present at the surface of the iron wire when a current is passed through the system, just as in the case of the platinum wire in the core-conductor experiments of Hermann. Matteucci, and Boruttau. Changes of polarization, however, can give rise to unlimited transmission only in so far as they form the condition of chemical effects which alter the electromotor properties of the surface layer and themselves cause further changes of polarization. The general conditions of transmission in protoplasmic systems will now be discussed briefly, with more particular reference to the case of nerve, where the phenomena of protoplasmic transmission appear to exhibit themselves under the simplest conditions. The fundamental problem, however, is the same for all forms of protoplasm.

According to the law of polar stimulation, the direction of the current between active and resting areas is such, relatively to the surface of the irritable element, that its normal physiological effects - assuming them to be the same as those of an external current led into the tissue-would be to initiate excitation at the resting region adjoining the excited area and to repress activity in the excited area itself. An inspection of the diagram (Fig. 4) will show this. The direction of the local binelectric current (relatively to the protoplasmic surface) at $R$ is the same as that of the external current at the 
cathode of a pair of stimulating electrodes. A current traversing the protoplasmic surface in this direction arises as soon as the local area of stimulation, $S$, is altered (e.g., by mechanical or chemical action) sufficiently to render it negative relatively to the areas adjoining. The appearance of this current, which acts at a distance from the directly altered area, is apparently the primary effect in stimulation. It initiates the propagated wavelike disturbance, because each secondarily stimulated

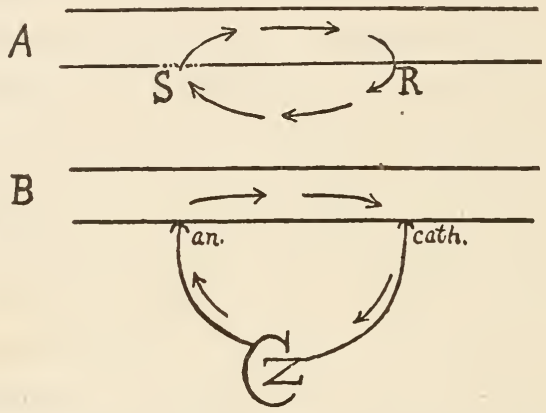

FIg. 4.-In $A$ the arrows represent the direction of the current in the active-inactive circuit on one side of the stimulated region $S$. In $B$ the course of an external stimulating current from a battery is represented. Stimulation originates, on make, at the cathodal region, where the current has the same direction, relatively to the membrane, as at $R$.

area, e.g., at region $R$, on itself becoming negative, produces automatically the same effect on regions beyond; and this effect is repeated at each boundary (or region of transition) between an active area and the resting area in immediate advance of it.

There is an analogy of a general kind between the spread of excitation over an irritable protoplasmic element and the spread of combustion along a fuse, with the difference that in the fuse the chemically active area initiates action in the adjoining area through the heat generated in the local reaction, while in the 
protoplasmic element transmission is effectal by the electric current flowing between the two adjacent nreas of different potential. In the fuse a temperature gradient exists between the burning area and the region arljoining. and the reaction begins wherever the emperature reiches the ignition point. The rate of transmi-ion in such a case depends on the maximal distance (from the boundary of the burning area) at which this critical temperature is reached and on the rate at which heat is locally deseloped; i.e., $V=K s r$ where $V$ is the speed of transmission, $s$ the maximal distance, and $r$ the rate at which temperature rises in the ignited area. ${ }^{\mathrm{I}}$ Similarly in the nerve atone (or other protoplasmic element) the velocity of transmission will be a direct function ( $\mathrm{I}$ ) of the maximal distance, $s$ (from the active area) at which the current of the local bioelectric circuit is effective as stimulus, and (2) of the rate, $r$, at which the current develops; again $V=K s r$. Instead of the rate of development, $r$. we may consider its reciprocal, the time, $t$, required for the current at the secondarily stimulated point at distance. $s$, to attain a stimulating value; the shorter this time the more rapid the transmission, i.e., $V=K s / 1{ }^{2}$

This equation also applies to the transmission in the passive iron model. Between the activated and the inactive areas of an iron wire immersed in dilute nitric

${ }^{x} K$ represents constant limiting factors, such at the rate at which heat is conducted or radiated from the burning region.

The distribution of temperature in the gradient on cither tile wf a heated area in a wire (or similar heat-conductor) is in fact inbject to the same quantitative law as the distribution of potchtial in a lexally

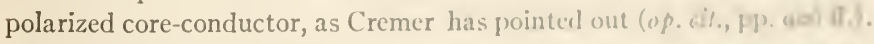

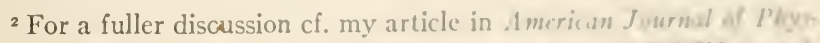

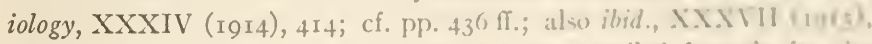
362 ff. Cremer has recently derived a more detailed formulis for tie 
acid there is a P.D. of about 0.7 volt; a local current flows between the two areas as indicated in Figure 3 (p. 253), the active area being anodal. ${ }^{\mathrm{I}}$ This current is most intense near the active-passive boundary, because the resistance of the portion of current traversing the surface at any point in the passive area increases with the distance of that point beyond the boundary; this resistance depends chiefly on the length and specific conductivity of the column of electrolyte intervening. Up to a certain critical distance, $s$, beyond the boundary the current will have sufficient intensity and local density to reduce the film; hence the time, $t$, required for the current to reach this intensity and the distance, $s$, from the boundary are the essential variables to be considered. ${ }^{2}$ The effect of increasing the resistance of the local circuit (and thus decreasing the distance, $s$ ) may be shown qualitatively by suspending the passive wire vertically, with a thin layer of acid adhering, and then touching it below with zinc. ${ }^{3}$ Under these condi-

velocity of transmission in nerve, introducing as factors the relative electrical resistances of axone and sheath and the specific electrical sensitivity of the tissue, as well as the rate of development of the electric variation. If certain reasonable assumptions are made, this formula agrees well with observation. Cremer's formula is consistent with the foregoing simpler expression, $V=K s / t$, but attempts to define more closely the conditions determining the value of $s$ (Ber. ges. Physiol., II [1920], r66; also Cremer's Beiträge zur Physiologie, II [1922], Heft I, 3I).

`Jour. Gen. Physiol., III (1920), I30.

2 Only the maximal distance from the boundary at which the current is effective need be considered, since both systems react in the "all or none" manner. This type of reaction is a necessary condition for indefinite transmission without decrement.

3 It may also be shown quantitatively by inclosing the wires in glass tubes of different diameters; the rate varies directly with the sectional area of the tube. 
tions the wave of activation (mar'ed by the darkam. ing of the metallic surface) moves slowly umwan as the rate of only a few centimeters per secomel; while in the case of a wire completcly immersed in a large volume of acid the transmission is too rupil to follow with the eye, i.c., some hundred centimeters per second.

Rapidity in the local variation of potential in bervo or other conducting tissue is thus a nccessury conditian for rapidity of transmission. The table given on prage 328 shows a general proportionality between the rate of development of the bioelectric currents in difierent tissues and the rate of transmission. The slowring of the bioelectric variation in a particular tissue, by cols, anæsthesia, or fatigue, involves a corresponding slowing in the transmission rate. An exact proportionality is hardly to be expected, because the other propertic of the tissue, especially its irritability and its electrical conductivity, are also affected by the change of condi tions, and other variables enter; but that the rate of electromotor variation is the chief factor determining the speed of transmission seems clearly indicated by the observations cited in the table.

With regard to the other variable, $s$, the distance from the active-resting boundary through which the local bioelectric current is effective as a stimulus, definite information is difficult to obtain. I have atteruptod to estimate this distance in frogs' nerve by menturing the maximal distance between two flitinum electmodes. applied to the tissue and having a I'. I). similas to that of the bioelectric variation (20 to 40 millivolis? at which stimulation occurs on the make and break of a 
constant current. ${ }^{x}$ With a P.D. of ten to twenty millivolts, stimulation occurs at either make or break with the electrodes fifteen to twenty millimeters apart. If we regard the conditions of resistance as similar in a stimulating circuit of this kind and in the normal bioelectric circuit, we may infer that the normal actioncurrent flowing between the active and the resting portions of an excited nerve is effective at a distance of three to four centimeters from the excited area. Other observations, by Hering and others, on secondary stimulation by the demarcation-current, support this conclusion. ${ }^{2}$

The local action-current in the frog's motor nerve reaches its maximum at about .OOI of a second after its initiation $\left(\right.$ at $20^{\circ}$ ); this is the duration of the rising phase of the action-current curve, according to the observations of Garten and others. ${ }^{3}$ If we assume that this current, at the moment of reaching its maximum, has a stimulating effect on all resting regions of the nerve within a distance of three centimeters from the active

${ }^{x}$ Op. cit. (rgI4), p. 433 .

${ }^{2}$ Cf. article just cited, p. 43 I. See also the recent observations of Spierling (Cremer's Beiträge zur Physiologie, I [19r8], Heft 7) and Keil (Z. für Biologie, LXXV [1922], I), on the minimal P.D. required for the stimulation of frog's nerves. In Keil's experiments electrodes of varying form were used, and the stretch of nerve traversed by the current varied between 0.5 and $4 \mathrm{~cm}$. in length. The values obtained were of a similar order to those found in my experiments just cited, but showed wide variation. The potentials required with stretches of nerve 2 to 4 $\mathrm{cm}$. long varied between 20 and $c a .300$ millivolts.

3 More recent determinations indicate a more rapid rise; cf. Gasser and Erlanger's observations with the cathode ray oscillograph (American Journal Physiology, LXII [1922], 496; also Plaut, Z. für Biol., LXXVIII ([1923], 133). 
area, we have a transmission of stimulating effect through three centimeters in .0OI of a secmint; this if equivalent to thirty meters per secont, the usual transmission-relecity at this temperature. The reuts of this simple calculation are thus in agrecmem with observation and support the view that transmiesion is in reality a case of secondary stimulation by the cument

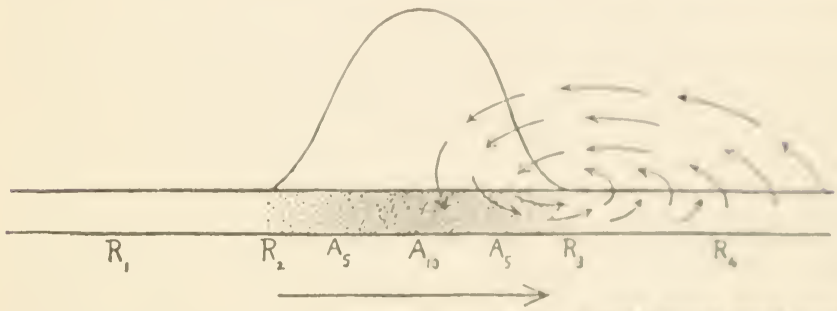

Fig. 5.-Diagram of the momentary condition in a frisk nemt aw anea

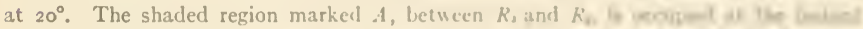

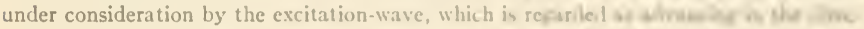

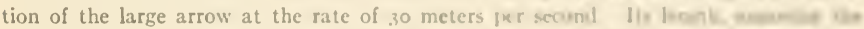
total duration of the local process (as inclicated hy the durathen of the focul terorre variation) to be .002 second, is $0 \mathrm{~cm}$. The excitati n-pirke is jun laslube at $R$. has reached its maximum at $A$ so, and has just sul ided at $k$. Toe cwse ondferest ile variation from the resting potential at different point in the a tive rukat dieneriese P.D., at $A_{\mathrm{ro}}$, is $c a .40$ millivolts. The regions markel $R$ are in the reiles atun The

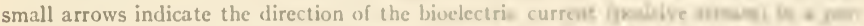

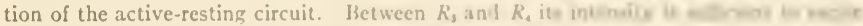
the nerve; excitation is thus always being initiated at a wasne f as in almoss al

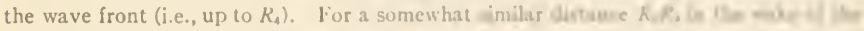
excitation-wave the nerve is refractory to stimulation.

of the local bioelectric circuit. Figure 5 gives a diagratotmatic representation of the conditions in a nerve during transmission.

The course of the current in the bioctectric circuit should be noted; this course is partly extra-cellular, i.e., through the medium, ${ }^{1}$ and partly intra-cellular,

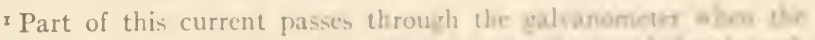

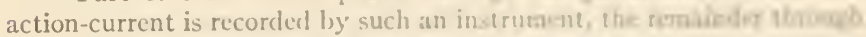
the medium or other extracellular conducting patti. 
the current at its regions of entrance and exit also traverses the plasma membrane. The current passing lengthwise along the fibers of a tissue like a nerve is undoubtedly subject to electrostatic retardation, as in the analogous case of a cable; and Crehore and Williams ${ }^{\mathrm{I}}$ have calculated that the speed with which its variations are transmitted would thus be reduced to a value similar to that of the nerve impulse. It is evident that an upper limit to the speed attainable by transmission of the kind above is set by the speed with which variations of currentintensity can be transmitted lengthwise along such a conductor, since the general physical conditions present in conductors with boundary surfaces having capacity are undoubtedly present in protoplasmic structures like nerves. Such conditions are common to all conducting paths having a certain structure (core-conductors). It would be erroneous, however, to infer that transmission in nerve is identical with transmission along a cable. The nerve impulse, or any other protoplasmic excitation-wave, is an active process whose energy is derived at each portion of its path from local chemical reactions. Its electrical component furnishes the conditions for transmission from each region to the next, but does not constitute the whole process in the physiological sense. Nevertheless the conclusion that certain physical factors are common to both systems must on general scientific grounds be regarded as correct.

Since a portion ("return path") of the bioelectric current traverses the external medium, we should expect that varying the electrical conductivity of the medium

${ }^{x}$ Crehore and Williams, Proceedings of the Society of Experimental Biology and Medicine, XI (1913), 59. 
would have a corresponding effect upon the speed of protoplasmic transmission. A lowering of the conduetivity of the local bioclectric circuit should invelse a corresponding decrease in this velocity, since the maxima! distance, $s$, at which the local current still has stimulationg intensity, would be proportionately decreased by amy increase of electrical resistance. This critical distance should, other conditions being equal, be proportional to the conductivity of the circuit. Mayor's experimemt on the rate of transmission in the nerve net of the medusa Cassiopea in dilute sea water show in fact that within a considerable range of dilutions (down to 50 volumes per cent sea water) a close proportionality exists between the salt-content of the medium and the transmission rate. ${ }^{\mathrm{X}}$ This result indicates a direct correlation of this rate with the electrical conductivity of the medium. 'The recent investigation of I'ond' on the speed of the contraction-wave in various forms of muscle (cardiac and voluntary of frog and heart of Limulus), using mixtures of balanced salt solution and isotonic sugar solution, has shown that in these tissues also the speed of transmission runs closely parallel with the electrical conductivity of the medium. The transfer of a muscle from a medium of low to one of high conductivity is followed by a corresponding increase in the speed of the contraction-wave, and vice versa. Mayor's and Pond's observations are difficult to explain except on the assumption that clectric currents traversing the cell-media are a chief factor determining the rate at

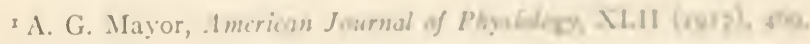
and XLIV, $59 \mathrm{r}$.

${ }^{2}$ S. E. Pond, Jour. Gen. Phy iol., III $(1(221)$ bof. 
which excitation is transmitted from one region of a protoplasmic element to another; this assumption, howerer, is a direct corollary of the local action theory of transmission. Further comparative observations in this field are desirable; the theoretical side also needs careful consideration, since both extracellular and intracellular conductivities are concerned and the precise relations between these two are insufficiently known. ${ }^{\mathrm{r}}$

Various other facts of comparative physiology also indicate the importance of the bioelectric variations in the different forms of protoplasmic transmission. The transmission of excitation between cells which are in contact or close proximity but not otherwise connected is a phenomenon difficult to explain except on the electrical theory; the "rheoscopic frog" experiments have already been cited as examples of transmission demonstrably resulting from secondary electric stimulation by bioelectric currents. Several years ago, I called attention to a number of instances of apparently the same effect; ${ }^{2}$ for example, one active swimming plate in a ctenophore can influence another through several millimeters of sea water; spermatozoa collected in a clump are soon found beating synchronously; ${ }^{3}$ ciliated epithelial cells transmit waves of movement; the trans-

I Brooks has recently studied the relation between the conductance of living cells and tissues (Laminaria, yeast, bacteria, Chlorella) and the conductivity of the medium, and finds a close proportionality between the two (Jour. Gen. Physiol., V [I923], 365.

${ }^{2}$ Op. cit. (I9I4), pp. $427 \mathrm{ff}$.

3 The detached cilia of Paramœcium show a similar behavior, according to recent obsrvations of $\mathrm{Al}$ verdes (Arch. ges. Physiol., CXCV [I922], 245). 
missions between neurones in the central nervous system and between nerve-endings and muscle cells are evidently by contact. Such transmissions point almost certainly to electrical conditions. The sensitivity of certain irritable elements to electrical currents in the surroundings has in some cases been developed to a remarkable degree; for example, the catfish will respond to the dipping of a metallic rod into the aquarium at a distance of several centimeters from the fish. It is possible that such animals may detect living prey' through the action-currents accompanying muscular movements.

Transmission of excitation or other physiological influence-implying transmission of chemical influence to a distance in protoplasm-may be called "physiological distance-action," after the analogy of "chemical distance-action"; the latter is an electrical phenomenon depending on the mutual influence of the electrode areas in circuits. $^{2}$ A simple example will illustrate. ${ }^{3}$ When a copper or platinum wire, e.g., 25 centimeters long, is immersed in a vessel of dilute $\mathrm{H}_{2} \mathrm{SO}_{4}$ and touched at one point with a piece of zinc, instantly bubbles of hydrogen start out from the surface of the wire along its entire length. The zinc forms the anode in the local circuit produced by the contact, the copper or platinum is the cathode, hence hydrogen is formed from the surface of the latter at all points where the current-intensity is

s Parker and von Heusen, a merican Journal of Phytiolagy, XLIN (1917), 405

${ }^{2}$ Cf. Ostwald, "Chemische Fernewirkung," \%. physik. Chem., Ix (1891), 540.

3 For other similar instances and a fuller discustion of the biological analogies cf. Biological Bullelin, XXXIII (1987), 235; cf. pp. 157 If. 


\section{PROTOPLASMIC ACTION AND NERVOUS ACTION}

sufficient. In other words, a reducing action comes instantly into play at a distance from the zinc as soon as the contact is made; this action depends on the passage of the current through the circuit, zinc, acid, and platinum. Similarly when a region of nerve is stimulated, that region becomes negative; and the presumption is that the current of the local circuit thus arising exerts chemical action at all points along the protoplasmic surface where its intensity is sufficient. According to the present theory, it is to this electrochemical action (which secondarily determines stimulation) that the transmission is due. Each area thus secondarily activated serves as a new point of departure for activation of the region beyond, and in this manner transmission to an indefinite distance becomes possible. As already pointed out, the conditions in the passive iron model are of the same general nature. The electrochemical modification of the surface layer (plasma membrane or passivating oxide film), through the electrolytic action of the local circuit, is in both systems the essential change determining transmission.

In the return to the resting or passive state after activation the current of the local circuit is also an essential factor; this current (as the diagram shows) passes in opposite directions (relatively to the surface) at active and resting regions; and correspondingly its physiological effect, which is excitatory at the resting region adjoining the region of activity, is anti-excitatory or inhibitory at the active region itself. Any region, on becoming active, is thus automatically subjected to an electrical influence which limits or arrests its activity. Hence the local activity, in a muscle cell or nerve fiber, 
is temporary, and the state of excitation appears to travel like a wave over the irritable element.

In the wire model the automatic return of passivity in strong $\mathrm{HNO}_{3}$ is the direct result of the formation of a new surface-film by electrochemical (oxidative) action at the local anodal regions. ${ }^{x}$ The phenomena of the refractory period in irritable tissues, and the observations already described showing that the plasma membrane of egg cells changes from a temporarily unstable to at stable state after cell-division or insemination, ${ }^{2}$ indicate that in living irritable elements also the essential condition of recovery after stimulation is the formation of a new surface-film, or the return of the altered film to its original condition. Other physiological facts support this view; e.g., the delay in the return of irritability. in a veratrinized muscle after stimulation is associated with a corresponding delay in the return phase of the bioelectric variation. 'The reversible change in the surface-film is the condition both of the normal bioelectric variation at any region and of the transmission of a similar change of state to adjoining regions.

The well-known interference with stimulation and transmission during the passage of a constant current lengthwise through a muscle or nerve is a further indication of the part played by electrical conditions in transmission. The region near the anode region of anelectrotonus) acts as a block to an excitation-wave; this effect is undoubtedly complex, but it is probable

${ }^{s}$ For a fuller account of the conditions in pasive mulub of. whe review of Bennett and Burnham, Journal of Phy iesl Chenitins, XXI (1917), 107.

${ }^{2}$ Cf. pp. 36 Iff. 
that it depends largely upon the direct physical compensation of the bioelectric current of the approaching excitation-wave, which in the region beyond the actual area of excitation traverses the surface in a direction opposed to that of the polarizing current (Fig. 6). An analogous effect is observed in a passive iron wire in nitric acid when a piece of platinum foil is pressed into close contact with it; an activation wave started

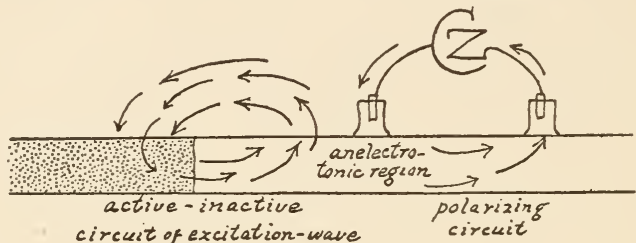

FIG. 6.- Showing the opposed directions of action current and polarizing current through the resting portion of the nerve in the anelectrotonic region; the excitation wave is nearing the anodal region of a battery current led into the nerve by non-polarizable electrodes.

at another region of the wire is blocked in the vicinity of the platinum. This effect is dependent on the intersection of the two local circuits, active-passive and platinum-passive, which are opposed in direction.

The mutual interference of excitation-waves in living tissues is probably to be explained in an essentially similar manner as an instance of mutual compensation of oppositely oriented bioelectric circuits. This phenomenon is best demonstrated in rings of medusa tissue $^{\mathrm{I}}$ or rings of heart muscle; ${ }^{2}$ two contraction-waves

${ }^{8}$ Cf. A. G. Mayor, Carnegie Institute Publications, No. 102 (1908), p. II5; American Journal of Physiology, XXXIX (19I6), 375; cf. p. 379 .

${ }^{2}$ W. E. Garrey, American Journal of Physiology, XXXIII (Igr4), 409. 
approaching each other from opposite directions undergo, mutual extinction where they mect. It is clear that the processes determining transmission are in some manner compensated or nullified where the waves meet: and since each wave is associated with a bioclectric circuit, and the two circuits, being equal and oppositely oriented, must physically compensate each other when superimposed, it is to be expected that any transmission of electric influence and hence of stimulating cfiect beyond their intersection will be impossible. The bloching of excitation-waves at regions of injury is probably. to be explained in a similar manner, as due to compensation of the action current by the injury current. ${ }^{\text {? }}$

The question of the part which interferences of thi kind play in the intact living organism (e.g., in the central nervous system) is an open one. Recently there have been attempts to explain physiological interferences such as those of reciprocal inhibition, the Wedensky phenomenon, vagus inhibition, etc., on the basis of a lack of correspondence between the normal rhythm of response and recovery in the receiving irritable system and the rhythm of the series of nervous impulses entering it from without. ${ }^{2}$ The Wedensky effect is an example of such a phenomenon, the fatilure of the muscle to respond after the initial contraction depending on the coincidence of periods of increased decrement in the motor end-plate with the periods at which the

Mayor refers the mutual extinction of interectiag wase to the refractory phase of the tissuc; "lissue which has been in contractuen cannot again contract until after an apprecialle interval of $x \mathrm{e}$ " Eloc trical compensation must, however, aloo be present, as in the sablegous cases of anclectrotonus and blocking at a region of injury.

${ }^{2}$ Keith Lucas, Conduction of the Nicrons Impulsen, clupse xlit sili 
successive waves of innervation are received. At each stimulation the decrement in the end-plate is increased temporarily. According to the degree of decrement an impulse received by the end-plate may penetrate the latter and reach the muscle cell with its normal "intensity," or it may undergo a decrease which may entirely prevent penetration. A grading of the intensity of innervation, depending on the degree of coincidence of phase in the natural rhythms of the two interconnected systems, is thus possible. Forbes has recently applied this conception to the case of sensory stimulation, and has expressed the view "that an unlimited range of sensory graduation might be based on the frequency with which the impulses follow one another in the sensory fibers." All of these possibilities should be fully studied and investigated, with a recognition of the probable dependence of excitation and transmission upon the bioelectric processes in the irritable elements. Further discussion of this problem is not possible in this place. ${ }^{2}$

The rôle of the bioelectric currents in other physiological processes (growth, cell-division, secretion, etc.) is only beginning to be understood, and much further investigation is required. It seems clear, however, that by means of these currents physiological influence may be transmitted rapidly to a distance in many protoplasmic systems other than nerve; and that correlations of activity and function may thus be effected which would otherwise be impossible. Appar-

× Cf. Forbes, Physiological Reviews, II (1922), 36r (cf. p. 388); Forbes and Gregg, American Journal of Physiology, XXXIX (I915), I72.

2 See Sherrington, "Some Aspects of Animal Mechanism," Nature, CX (1922), 346 . 
ently this general condition is illustrated in the phenomena of growth and development. There is evidence that many cases of form-correlation in both plants and animals have an electrical basis. Actively growing regions of organisms have usually been found electrically negative to more slowly growing regions. Hermann and Müller-Hettlingen ${ }^{\mathrm{I}}$ observed that in seedlings the regions near the growing zones (terminal buds and roottips) were negative to those near the cotyledons; similarly the growing zones of planarians and annelids are negative to intermediate regions, ${ }^{2}$ and in hydroids regenerating hydranth heads are negative to the stems. ${ }^{3}$ In general the indications are that regions of active constructive metabolism - which are usually regions of active oxidation - are typically negative to less active regions. Miss Hyde's observations on fish eggs ${ }^{4}$ indicate that during cell-division the cell body undergoes a temporary negative variation, analogous to that accompanying excitation; the electrical negativity of regions in active proliferation may thus be accounted for.

The presence of bioelectric circuits between the rapidly growing regions of an organism and the regions: adjoining is in all probability an important if not the chief factor in the controlling influence ("physiological dominance") which the former exerts upon the litter. In plants it has long been known that the removal of

¿Hermann, Arch. ges. Physiol., XXV'II (1SS2), 2Ss; MullerHettlingen, ibid., XXXI (1883), 193.

${ }^{2}$ Cf. Child, Biological Bulletin, XLI (1921), 90; Hyman and Bellamy, ibid., XLIII (1922), 3тз.

3 Mathews, American Journal of Physiology, VIII (1903), 294. Lund, Journal of Experimental Zoölogy, XXXVI (1922), 477.

4 I. H. Hyde, American Journal of Physiology, XI (1904), 52. 
growing regions (terminal buds) initiates growth in the dormant regions adjacent (axillary buds, etc.); any arrest of growth by cold, anæsthetization, or removal of oxygen has the same effect. ${ }^{r}$ Such facts show that pronounced activity of growth-processes in one region in some manner involves repression of similar processes in neighboring regions; and this effect has been shown in certain cases to be independent of the transport of special growth-inhibiting substances between the two regions. ${ }^{2}$ A similar inhibitory influence of one embryonic area on another is seen also in the development of animals. ${ }^{3}$

The hypothesis that the essential basis of this growthcontrolling influence is electrical is consistent with the observation that in certain organisms the rate and direction of growth can be experimentally controlled by electric currents. We have seen that in hydroid stems the growing or regenerating hydranths are negative relatively to other regions of the stem and to the stolons. Apparently, therefore, a normal accompaniment of growth is the passage of electric currents in a constant direction through the organism; and the direction of the current through the galvanometer shows that the positive stream enters the living system (from the exterior) at the regions where growth is most rapid. If electric currents are in themselves a factor in the

I McCallum, "Regeneration in Plants," Bot. Gaz., XL (1905), 97, 24 I.

${ }^{2}$ Child and Bellamy, Science, L (1919), 362; Bot. Gaz., LXX (I920), 249; Harvey, American Naturalist, LIV (I920), 362.

${ }_{3}$ Cf. Stockard, American Journal of Anatomy, XXVIII (I92I), I 5 . 
growth process, it is to be expected that their passage through the organism from without should either promote or inhibit growth according to the direction of flow. Lund has in fact recently shown that the regeneration of new polyps from the cut stems of the hydroid Obelia may be controlled by weak electric currents passed lengthwise through the stems; ${ }^{\mathrm{r}}$ the formation of hydranths is promoted where the current passes so as to enter the protoplasm from the medium-i.e., at the cut end facing the positive pole of the battery-and inhibited at the other end. The normal polarity of a stem can thus be reversed by passing the current, a result in agreement with the view previously expressed by Mathews that morphological polarity in these organisms has an electrical basis. ${ }^{2}$ Bose has also found that the electric current influences growth processes in the higher plants in a polar manner, the anode enhancing and the cathode depressing the normal rate. ${ }^{3}$ Recently Ingvar has reported experiments in which the outgrowth of processes from embryonic nerve cells is influenced in a directive manner by the passage of weak currents through the culture medium. Here also a polar influence is seen, the processes growing toward the anode differing morphologically from those growing toward the cathode. All of these facts show clearly that growth processes resemble the processes of stimulation in irritable tissues in being subject to electrical control; further that in this

${ }^{x}$ Lund, Journal of Experimental Zoölogy, XXXIV (1921), 478 .

${ }^{2}$ Mathews, loc. cit.

3 Bose, Procecdings of the Royal Socicty, B, XC (1918), 364.

4 Ingvar, Procecdings of the Society of Experimental Biology and Medicine, XVII (1920), 199. 
control the polar influence is all-important. ${ }^{\mathbf{x}}$ If what is true of artificial electric currents is true also of the currents produced by the living system in its own activity, the conclusion seems unavoidable that the bioelectric currents exert a controlling and co-ordinating influence in normal growth processes as well as in normal stimulation. ${ }^{2}$

Perhaps the most important general inference to be drawn from such experiments is that the electric current under appropriate conditions has a direct promoting influence on the synthetic reactions in living matter, i.e., those underlying growth and repair, as well as on the reactions involving oxidation and decomposition which yield the energy for normal activity. Many years ago, before the development of modern physical chemistry, Hering reached certain general conceptions of the relation of the current to protoplasmic action resembling closely in many respects those reached as the result of our present analysis. ${ }^{3}$ Hering regards the bioelectric currents as essentially an index of chemical

In a recent paper, I have called attention to a number of analogies between organic growth and the growth of precipitation-structures on metals ( $\mathrm{Zn}, \mathrm{Fe}, \mathrm{Co}$, etc.) in ferricyanide solutions; control by electrical conditions is also characteristic of such precipitation-growths (Biologicai Bulletin [I9I 7], loc. cit.; cf. pp. I62 ff.).

${ }^{2}$ According to Kappers, the direction of outgrowth of nerve-tracts in the central nervous system is the expression of a directive electrical influence (analogous to galvanotropism) which he calls "neurobiotaxis." Cf. Kappers, "On Structural Laws in the Nervous System," Brain, XLIV (I92I), I25, and earlier references there given. Cf. also Child's discussion in his Origin and Development of the Nervous System, University of Chicago Press (I92I), chaps. x, xi.

${ }^{3}$ Hering, Lotos, IX, Prag (I888), translated in Brain, XX (I897), 232. 
reactions occurring in the protoplasm; conversely, electric currents passing through protoplasm from without alter its activity through their direct influence on its chemical processes. Hering also concludes, from the contrast between the physiological effects at the two electrodes, that where the current enters protoplasm from the surroundings it induces or promotes assimilatory (anabolic) processes, and where it leaves, dissimilatory. (catabolic) processes. In the typical irritable tissue the inhibitory effect at the anode is the expression of at predominance of anabolic processes, while the stimulation at the cathode results from chemical effects of the reverse kind (predominantly catabolic).

From our present point of view the above-described influences on growth and regeneration point to the conclusion that where the current enters the protoplasmic surface from the exterior it has the effect of promoting oxidation processes which form secondarily the condition of the syntheses required for the formation of new structure. Growth, repair, and recovery from stimulation are the result or expression of chemical reactions of the same general kind, apparently oxidative syntheses, which occur predominantly at the one polar region. It the other polar region reactions of the reverse kind are promoted: these form the condition for stimulation in irritable tissues or for cessation of growth or regression in growing regions.

The closest physico-chemical analogies to suetr processes are furnished by the chemical effects at clectrodes, i.e., the phenomena of electrolysis; and the possibility that electrolysis may underlic the physiological effects of the electric current was in fact early recognized 
by $\mathrm{Du}$ Bois-Reymond and other physiologists. ${ }^{\mathrm{I}}$ Evidently the fundamental problem to be solved is the problem of the physico-chemical basis of electrical sensitivity in living matter. The various facts and considerations reviewed in the foregoing chapters indicate that this basis is to be found in the characteristic physical structure of protoplasm; in other words, that the polyphasic and film-partitioned feature of the system is the condition which makes it possible for the electric current to produce such definite chemical effects. The polar action of the current is a clear analogy to electrode action, and we have to inquire into the justification of regarding the protoplasmic surfaces as having properties like those of electrode surfaces in general. At the surface of a metallic electrode, chemical action occurs when the current passes between metal and solution; and apparently the same occurs when a current passes from one protoplasmic phase to another; e.g., during electrical stimulation from the surface of the plasma membrane to the adjoining medium. As we have seen, the passage of the current (positive stream) in this direction is the condition of stimulation in an irritable cell or nerve fiber.

The parallels between the surface of a metallic electrode and the surface of a living cell or organic membrane have already been discussed in part, and certain resemblances have been pointed out. It remains to be seen whether the two are similar in the further respect that the passage of an electric current across

${ }^{2}$ Cf. Du Bois-Reymond, Untersuchungen über tierische Elektricität, II, 387 ("galvanische Reizung ist uns nichts mehr als die erste Stufe der Elektrolyse eines Nerven"). 
the surface is in both cases attended with chemical change. The chief peculiarity of the chemical reactions occurring under the influence of electric currents in electro-chemical circuits is that they are confined to the boundary region, where the current passes between the electrode and the electrolyte solution. No chemical change occurs in the interior of either phase; the reactions are surface reactions. Since the region of trancition from the metallic conductor to the electrolytic conductor is the only region in the circuit where the passage of the current involves chemical change, the fundamental question relates to the general physical nature of the conditions in this region while the current is tlowing. According to modern physico-chemical theory, the carriers of the current in the electrolyte solution are the anions and cations of the dissociated electrolyte; in the metal the carriers are free electrons. At the surface of contact there is a transfer of electrons between the electrode and the ions of the solution. For example, at the anode, ferrous ions are oxidized to ferric ions; on the electron theory this implies the transfer of an clectron from each ferrous ion to the electrode; conversely; at the cathode electrons are transferred from the metal to the ions in solution, $\mathrm{H}$ ions becoming uncharged $\mathrm{H}$ atoms. To effect this transfer a certain potential gradient is required; it would appear therefore that the region of transition represents that portion of the circuit where the potential gradient is steepest and where the forces acting to displace electrons are greatest. That the contact of two dissimilar conductors, respectively metallic and electrolytic, is not the essential condition, but rather the existence of a large fall of potential across a short distance, 
is indicated by the facts of electrostenolysis and by the chemical effects produced by intense electrical discharges (sparking, etc.).

The general fact that chemical effects are produced in protoplasm by the electric current and that protoplasm produces electric currents in its activity, when considered in conjunction with the further fact that these phenomena are dependent on the structure of the living system and disappear with the loss of semi-permeability (as at death) - as well as the various other facts reviewed above, which relate stimulation to membrane changes-indicates clearly that the electrical sensitivity of living protoplasm is intimately connected with the presence of the semipermeable partitions or surface-films. These partitions have high electrical resistance and are therefore highly polarizable; they are also extremely thin; hence when they are polarized by the passage of a current there is a correspondingly steep fall of potential between their opposite faces. The hypothesis naturally suggests itself that the existence of these steep gradients is the essential condition on which the chemical action of the electric current in living protoplasm depends.

We have recently attempted to put this hypothesis to an experimental test by passing electric currents through electrolyte solutions partitioned by artificial membranes, combining extreme thinness with high electrical resistance. ${ }^{I}$ Such a membrane, consisting of a thin film of rubber or other insoluble non-conducting material

${ }^{x}$ R. S. Lillie and S. E. Pond, "Chemical Effects Produced by Passing Electric Currents through Thin Artificial Membranes of High Electrical Resistance," American Journal of Physiology (1923); Proceedings of the American Physiological Society, December (1922). 
supported in a sheet of lens paper, is interposed between two salt solutions, one of which contains a readily oxidizable compound, ferrous chloride, together with in indicator (KCNS) to show the formation of ferric ions. With membranes of about $50 \mu$ thickness and a P.D. of about I I volts between the two faces, the red color of ferric thiocyanate appears rapidly at the surface facing the cathode; i.e., where the positive stream of the current passes from the membrane to the solution. The surface of the membrane under these conditions acts (in the qualitative sense) like the surface of a platinum anode. When the direction of the current is reversed, ferric ions are reduced to the ferrous state, as shown by the gradual disappearance of the color. In order to obtain these electrolysis-like effects, a certain minimal P.D. (i.e., steepness of gradient) across the membrane is required; for example, with a current giving 9 volts

- There is an interesting analogy here with the conditions of electro stenolysis, where also a critical P.I). is required for producing cliemical effects. In the experiments of Braun ( $A \mathrm{~m}$. d. Physik. XIII $\mid \mathrm{ISp} 1$, N.F., 473) thin sheets of mica were used (ca So $\mu$ thick) in which tis ures were cut; metallic silver separates out rapidly at the borders of tie fiscure when a strong current is passed through such a sheet separating two solutions of $\mathrm{AgNO}_{3}$. Braun compares such chemical effects with Bee querel's "electrocapillary reactions" (cf. Comples rondus, LXXiT [1873], 1037) and suggests that they may have bielogical significanor: he regards a narrow split or fissure in a thin layer of insulating materbl as. acting essentially like an elcctrode; a certain critical intensity of current is required, below which there is no separation of metal. Ierese the fissure there is a steep fall of potential, which he cotimate at $;$; volts per millimeter in currents effective with $\mathrm{Ag} \mathrm{NO}_{\mathrm{J}}$.

For observations on chemical effects at precipitation memalranis through which electric currents are pased cf. Ostwald, Z. phytik. Cles . VI (I890), 7I; Overbeck, Am. d. Physik, Xl.1I (1501), roj: Spriagmann, ibid., LI (1894), I40; Bein, Z. physik. (hem., .XVVIII (1 wewa). 439 . 
P.D. across the membrane, no effect is obtained in half an hour or more. The effective gradient of about Io volts, across a partition $50 \mu$ thick, is equivalent to a fall of about 2,000 volts per centimeter.

From these experiments we may conclude that an arrangement of electrolyte solutions containing oxidizable materials and partitioned by thin films of water-insoluble material having high electrical resistance will be the seat of chemical change (oxidations and reductions) occurring at the surface of the partitions when the system is traversed by an electric current of sufficient intensity. It seems probable that this kind of structural arrangement is the one upon which the electrical sensitivity of living matter depends. We have already seen that this type of structure is characteristic of living protoplasm. The potentials in protoplasm are much smaller than those used in the experiment above; apparently 50 to Ioo millivolts is the usual order of the variations of potential in the bioelectric processes; but the protoplasmic films are much thinner than those used in our model; and since the steepness of the gradients rather than the absolute values of the potentials is the essential factor to be considered, the conclusion seems justified that conditions similar to the above exist in living protoplasm when the system is traversed by a current.

The parallel between the passive iron system and an irritable protoplasmic system such as a nerve axone may be described in general terms as follows. In both cases there are two electrically conducting phases separated by a thin impermeable film of chemically alterable material. In protoplasm both of the phases are electrolytic conductors. In the passive iron system only one 
phase is electrolytic, the other being metallic; but the chemical reaction, which is confined to the interfacial layer, does not depend directly on the internal compusition and physical properties of either phase but only upon the conditions at the interface. When a current of sufficient intensity passes across the boundary at any region, in either system, it causes polarization and pro duces chemical effects; and under the conditions already defined, these effects may be automatically transmitted over the whole surface.

Such a conception of protoplasmic structure and action is fully consistent with the views reached on the basis of histological research, and it has the further advantage of correlating the structural features of the living system with the special peculiarities of its chemical and physiological behavior. The great diversity exhil)ited by living organisms shows that the protoplasmic type of constitution permits the widest variation in the details of structure and activity. Yet the essential or fundamental structure common to all forms of protoplasm is apparently uniform; viz., a film-partitioned or filmbounded arrangement or organization of phases of different chemical composition. With this type of structure, of which an elementary model is an emulsion structure. the properties of growth, chemical activity, and irritability characteristic of living matter appear to be intimately bound up. Systems having this structure will give a maximum of polarization when traversed by electric currents, and hence a maximum of chemical effect. Most of the problems relating to the mode of action of such systems, especially the problem of the conditions of specific synthesis (the most characteristic 
property of living matter), are still unsolved. The study of artificial systems having a similar type of physical constitution may be expected to throw further light on the nature of protoplasmic action, and also to indicate the directions in which further physiological research is most desirable. 


\section{INDEX}

\section{N. C. State College}

Absorption, 94, 102, 110

Action currents, 3Ir ff. Sce Bioelectric variations

Activation, changes of permeability and, 353 ff.; of egg cells, $74,265,353 \mathrm{ff}$; of passive iron, 254; by salt solutions, $35 \mathrm{I}$

Adaptation, 2, 8, 9, 30; active, 9, 30; static, 9

Adsorption, 23, 63, 75 ff.; catalysis and, $76,83,91,239$ ff.; chemical reactions and, 83,$90 ;$ electrical factors in, 84 ; films, 58 ; irreversible, $76,77,82 ;$ narcotic action and, 96, $20 \mathrm{r}$ ff., 233; potentials, $88,93,300$; selective, 79 ; specific, $78,79,82$

Agglutination, 163,208

"All or none" reactions, 324, 386 Alternating currents, activation by, 257; stimulating action of, 278,357

Anæsthesia (see also Narcosis), $40,42,47,83$, I 42 , I 89 ff., 263; by electric current (sce Electrotonus); relation to permeability, 353,363

Anaphylaxis, 35, 266, 370

Animals and plants, 12,28

Antagonisms, by salts, 155, 157, I 58, I 64 ff., I 76, I 8 I ff., 351 ; between salts and anxsthetics, 2 I, 352

Anticatalysis, 202, 226 ff., 236

Antigenic properties, relation to specificity, 34, 35, 36, 37

Antitoxic action, of salts, ${ }_{6} 6 \mathrm{ff}$.; of anæsthetics, $211,35^{2}$

Asymmetry, chemical, specificity. and, 34

Autocataly'sis, 36

Autolysis, 44, 48, 56, 57, 377
Bacteria, 14

Bioclectric potentials, memirane theories of, $302 \pi$.; relation to permeability of membrane 30r ff., $311 \mathrm{ff}$.; resting, $202 \mathrm{ff}$. variations with activity, $31 \pi \pi$

Bioclectric variations, 311 ff ; range, $324 ;$ rate in relation 26 phy'siological proce'sses, $320,32 \mathrm{I}$ $322,326 \mathrm{fl}$; relation to tranmission, 322, 330, 35; I1, rhythm of, 330 ff.

Bioluminescence, 375

Blood corpuscles, isoclectric point of, 100

Calcium salts, 45 , i1 9 , 155 fif.; antitoxic or stabilizing ection, 35 Iff., 369; infuence on permeability, I19; relation (1) excitation processes, 290; summation-interval and, 292

Capillarity, 77

Catalysis, 62, $6_{3}, 2<2$ ! $21 ; \pi$ i , 223,226 fi.; adsorption and. 223 If., 239 ff.; by charc 4 , 243,245 ; contact, $216,222 \pi$; $243 \mathrm{ff}$; electric factors in, 245 . $246 \mathrm{ff}$.; influence of surface active compounds on, $220 \pi$ by platinum, 23i, 2.45; rhvtlomical, 63,247 ff., 295 ; role al surface-films in, $247 \pi$.

Cataphoresis, 91, 99, 300

Cell, I4, I5 If., 67,05, rok. division, $3_{16}\left(1,35^{(6,}, 3^{6}, 3\right.$

Cell-divi-ion, $316,35 \%, 36 s$ : elce trical variation during, $3^{2} 4,306$. 399

Chemical reactions, rliydiesical 247,250

Chemical sen itivity, 46,47, =10

Chromosomes, A, 7 
Chronaxie, 275 ff., 287 ff., 326 , 327 ; relation to refractory period, 338

Cilia, 59, I6 5

Circuits, bioelectric, as factors in transmission, $382 \mathrm{ff}$; local, 63 , $221,236,260,27 \mathrm{I}, 274$

Colloids, 68, 69, 8r, $85 \mathrm{ff}$.

Compensation, of bioelectric currents in protoplasm, 397

Conduction, of excitation, etc. See Transmission

Conductivity, electrical, of artificial membranes, I79; of cellinterior, $\mathrm{I} 22 \mathrm{ff}$;; changes during activation or stimulation, 356 , $36 \mathrm{I}$; of living cells, II8, II9, I $22 \mathrm{ff}$., I30, 358, 359; of medium in relation to transmission, $390 \mathrm{ff}$.

Contact potentials, $236 \mathrm{ff}$.

Contractile processes, 59, 60, 378

Convection, electrical, of cells, 9I, 99, 300

Core-conductors, 380,390

Correlation, II, of growth, 4I, 42, 399 if.

Cortical reaction, of egg-cells, 360,362

Crystal forms, 32, 33, 34

Crystallization, relation to growth, 79, 225

Ctenophore, swimming plate of, $45,59,60,367$

Cyclopia, 39, 40

Cytolysis, 58, 74, I66, I99, 208, 355

Cytolytic agents, 57, 59; activation by, 355 ; stimulation by, 35 I ff., 366, 367

Cytoplasm, relations between nucleus and, I 7

Death process, $48,54,56,58,59,64$

Decrement, transmission with, 397,398

Demarcation potentials, I6o, $306 \mathrm{ff}$.
Depolarization, relation to stimulation, 283,284

Development, 6, 7, 62; experimental control of, $38,39,40$

Diffusion, 2I, 48, 57, 102; as factor in electrical stimulation, 279, 294; potentials, 303,307,319

Disintegration, polar, 277,360

Distance-action, chemical, 260, 393; physiological, 393, $398 \mathrm{ff}$.

Dominance, physiological, 399

Egg-cells, changes of permeability during activation, $360 \mathrm{ff}$.

Electric organs, 3I 7

Electrical activation, of passive iron, 254, 255

Electrical conductivity, of artificial membranes, I79; of cells, II8, II9, I22 ff., I30, 358, 359 ; of cells during activation or stimulation, $35^{6}, 36 \mathrm{I}$; of external medium in relation to speed of transmission, $390 \mathrm{ff}$.

Electrical convection, 91, 99, 300

Electrical endosmose, 94, I47, I48, 300,301

Electrical sensitivity, of protoplasm, 46, 47

Electrical stimulation, 273, $274 \mathrm{ff}$.; secondary, $388,389,392$

Electrocatalysis, 220, 236

Electrode potentials, 304

Electrolysis, 63, 219, 220, $235 \mathrm{ff}$., 252 ff., $277,282,298,403,406$; intermittent, 250; as factor in transmission, 253,382 ; at surface of membranes, 407

Electrolytic oxidations and reductions, 282

Electrostatic conditions, in nerve transmission, 390

Electrostenolysis, 406, 407

Electrotonic currents, 358

Electrotonus, 256, 277, $395 \mathrm{ff}$.

Emulsions, 67, 68, 70 ff., 98, ro4, I 77 
Environment, relations of organisms to, $25 \mathrm{ff}$.; as source of stimuli, $26_{3}$

Enzymes, 5t, 56, 222 fi., 2.10 ff.; specific action of, 224

Equilibration, 10, 26

Equilibrium, vital, $4 \$$, t9

Excitation. Scc Stimulation

Explosive corpuscles, 270,349

Fatigue, structural changes in, 61 Fermentation, alcoholic, 53, 226

Films, interfacial, $7 \mathrm{x}, 72,78,00$, 98, 104, 105, 106, 177; relation to activation, 298 ; relation to rhythmical chemical action, $247 \mathrm{ff}$.

Films, protoplasmic, formation of, I $26 \mathrm{ff}$., $35^{\circ}$

Film-structure, changes during stimulation, $3+6 \mathrm{ff}$; importance in protoplasm, 60, 101, 104, 126, 127,128

Foam structure, 70, 71, 74

Form-correlation, 399

Form-determination, 32

Formative metabolism, $6_{5}$

Fuse, transmission in, $3^{8}+$

Galıanotropism, 277

Gelation, by narcotics, 228, 229

Gels, 70, 106, 107

Genes, 6, 7

Growth, 2, 4, 5, 36, 38, 42, 70; action of narcotics on, +2 ; bioelectric phenomena of, 39. . 399; crystalline, 79. 225; dependence on oxidations, to.3; dependence on specific synthe is. $+2 \mathrm{ff}, 54,217$; polar infuence of electric current on, $40 r$; relations to normal physiological activity, $+\mathrm{r}$ fi.

Hæmoglobin, crystal forms of, 33 Hxmolysis, 99, I(3 $3,109,208,21.3$ Heat-production, 50
Heredity, 2, 4. \&, 1, 27, 49, th: factors of, 39, 30, relation to growth, $4,27,38$

IIomologous series, phy iulogical action of, 111,107

Hormones, as factors in integratirin, $7, \mathrm{tt}$

Hydrogen ion concentration, 85 , S., Sc), 93

Hydrolysis, 4s, 53

Inhibition, 45, 59, 62, 304, 307; of growth, 400

Injury currents, 20); influence of aniesthetics on, oor)

Inorganic salts, phy=iological action of, 151 ff. Se Salte

Instincts, 10, II

Integration, 0, 10, 11, 259

Interfaces, protoplasmic, $21 \%, 26$; relition to cluemical reactions in cell, 217 If. See Films

Interference, of excitation-waves. 306 II.

Ions, physiological action of. I5 $\mathrm{I}$ ff. Sec Silts

Ion-antagonisms, 02, 155 fi. Sc Salts

Iron, passive, activation of. JS4, 255; recovery of tran-mis-ivity in, $345,34 f$; summation effects in, 255 ; transmision in, 25. 8 . $260, \quad 270$ ff., $273, \quad 205, \quad 316$. $3 \Omega_{5}$ tif., 396$), 40.5$

Irritability, $2, \quad \mathrm{~A}, 30,40,43,41$ 102; rolation of salts to, $15,3 \pi$

Isocapillary solutions, 195 II.

Isodectric point, 85, \$o. v51 95. 100; of cells, 100

Kernleiter thenry, 3ro if.

\section{Lecithin, properties of anppersions} 225 if

light, stimulation bs, 266

light-prexluction, by oriarivant. relation to stimulation, 575 
Lipoids, presence in protoplasmic films, I84 ff.; as protoplasmic constituents, 58,67 , II I, I $84 \mathrm{ff}$; relation to narcosis, 83 , I $89 \mathrm{ff}$., $2 \mathrm{I} 4$; relation to permeability, I I I ff.

Lipoid-alterant compounds, action of, 56, 58, III, $187 \mathrm{ff}$.

Lipoid-solubility, relation to physiological action of compounds, I $89 \mathrm{ff}$.

Lithium salts, as substitutes for $\mathrm{Na}$-salts, 40, I54, I6 I

Living matter, general characters of, I ff.

Local action, 382

Local circuits, $236,245,246,250$, 253; in rhythmical catalysis, 250 : in transmission in passive iron, 253

Local currents, rôle in transmission, $386 \mathrm{ff}$.

\section{Maintenance, 2, 3, 65}

Membrane potentials, 107, $306 \mathrm{ff}$.

Membrane theory, of bioelectric potentials, $302 \mathrm{ff}$.

Membranes, artificial, I43 ff., I 85 ; electrical conductivity of, I 79

Membranes, changes during stimulation, $207 \mathrm{ff} ., 29.7,302,337 \mathrm{ff}$.; $346 \mathrm{ff}$.; electrical polarization of, I 59,30 Iff.; permeability of, Io9 ff., I 32 ff.; plasma, 20, 2I, $22,56,57,98 \mathrm{ff}$, IOI ff., IO9 ff., I59, I60, I 70; stabilization during narcosis, $207 \mathrm{ff}$.; structure, I8o. See Films

Membranes, haptogen, 105; porcelaine, I37; precipitation, I 36

Mercury, catalytic action of, 247

Metabolism, electrical factors in, 402, 403; formative, 7; general rôle in living matter, $2,3,4$, $25 \mathrm{ff}$., $42 \mathrm{ff}$; relation to protoplasmic structure, $5 \mathrm{I}$ ff., 63 ; synthetic, relation to carbohydrate metabolism, 54
Microdissection, 64 , I 28

Muscle, 50, 55, I $52 \mathrm{ff} ., \quad \mathrm{I} 62$; contraction of, 378 ; smooth, I $72 \mathrm{ff}$.

Myoneural junctions, ${ }_{5} 6$

Narcosis, I $89 \mathrm{ff}$.; adsorption and, 196, 20I ff., 233; changes in protoplasmic viscosity during, 210; decrease of permeability during, 209; oxygen-consumption in, I95, $203 \mathrm{ff}$.; partitioncoefficients and, I9o ff.; temperature and, I93 ff.; physical changes in protoplasm during, $206 \mathrm{ff}$.; surface-activity and, I $96 \mathrm{ff}$.

Negative osmosis, I47, 148

Nerve, 26r, 270; bioelectric variations of, $328 \mathrm{ff}$; changes of permeability during activity, $357 \mathrm{ff}$.; demarcation potential of, $303 \mathrm{ff}$.; electrical stimulation of, $267 \mathrm{ff}$;; electrotonic currents of, $35^{8}$; refractory period of, $339 \mathrm{ffi}$; relation of salts to irritability of, I 55 ; transmission, $379 \mathrm{ff}$.

Nerve cells, rhythmical activity of, 332 ; structural changes in death, 64

Nerve end-plates, 272,287

Nervous impulse, $379 \mathrm{ff}$.

Nervous system, directive factors in growth of, 402; integrative action of, II; neurone theory of, 272

Neurones, 272

Non-electrolytes, physiological effects of solutions of, I 5 I ff.

Nutrition, 2, 3, 6, 12

Organisms, general characters of, I, 2, 25 ff., 49

Organization, chemical, as dependent on film-partitioned structure of protoplasm, 22

Oxidases, 376

Oxidations, electrolytic, 237,246 
Oxidations, protoplasmic, $48,5 \circ$, $5 \mathrm{I}, 53,55$; relation to structure, $55,206 \mathrm{n}$.; relation to synthesis, growth, recovery, etc., 403

Oxygen-consumption, influence of narcotics on, 195, $203 \mathrm{ff}$.; influence of salts on, i is

Parthenogenesis, artificial, 352 ff.

Partition-coefficients, relation to narcotic action, $8_{3}$, 190 ff.

Partition method, of determining permeability, I 5

Passivity in metals, 254. Sce Iron, passive

Permeability, Ior ff., $\quad 132$ fl; changes in relation to stimulation and activation, 207 fी., 297, $302,337 \mathrm{ff}$., $346 \mathrm{ff}$; electrical conditions in, $146 \mathrm{ff}$.; factors in, 133; influence of narcotics on, 207,209 ; influence of salts on, r $74 \mathrm{ff}$.; methods of determining, I 2 ff.; physiological, I03, I 10, 134; of plasna membranes, $106 \mathrm{ff}$., $132 \mathrm{ff} ., 154,163,17 \mathrm{I}$, I $78 \mathrm{ff}$.; relation of chemical composition to, $143 \mathrm{ff}$.; relation of structure to, $135 \mathrm{ff}$.

Phase-boundaries, 22, 23; catalytic effects at, 244; protoplasmic, 192 , 196

Phase-relations, in emulsions, reversal of, 178,179

Photocatalysis, 235

Physiology, scope of, 2

Plasmolysis, II3, II 4

Platinum, catalytic action of, 237, 245

Polar disintegration, 277,360

Polar secretion, 360

Polar stimulation, $3 s_{3} \mathrm{ff}$.

Polarity, morphological, clectrical factors in, $400 \mathrm{ff}$.

Polarizability, changes of, during stimulation, $357 \mathrm{ff}$; of living protoplasm, 280, 357,358 ; relation to semi-permeability; 297
Polarization, electrical, 99, 101. 150, Is1, 27tff.; relation (i) stimulation, 273,278 ff., 3 sen 16 .

Polarization, physiological, afs. $3+4,3 s i$

Polarization-currents, allo, abf, 357

Potassium salts, $150 \overline{1}$.

Potential, chemicisl, 219

Potential, electric, 210; variation during periodic catalysis, $2.40 \pi$.; variations during tran=mission in passive iron, $253,205,344$; variations in living cells. Ses Bioclectric variations

Protective action, of collaids 23 I ff.

Proteins, relation to specificity, 3I ff.

Protoplasm, electrical sensitirit! of, 40,27+ If.; genera! characters, I II.; physical characters of, 48 fi.; reactivity of, 44 fi.. $259 \mathrm{ff}$.; specific syntles is in, 35 ff.; structural changes during activity or stimulation, $34^{5} \mathrm{ff}$; structure of, $19,5^{0}, 57,5^{8}$ 6 I ff., 66 ff.

Radionctivity, 172

Reactivity, of living matter, 20 30,43 II. Sic Irritability.

Recertors, 200

Refractory period, $1=7, \quad 207$; $337 \mathrm{ff}$; intluence of chemical conditions on, 340; in plane. 374 ; relation of merolirane changes to, 344 , $305 ;$ r relutions (1) bioelectric variatiun, 58 II:: temperature coeficicot, 341 . time-relations, 3.10

Regeneration, 5, 17, 401, relation to nucleus, 17

Regulation, 2, 9, 10 :\%, jo: 25 general jrojerty of stationsers systems, 50

Reproduction, 2, 45 


\section{I6 PROTOPLASMIC ACTION AND NERVOUS ACTION}

Resistance, electrical, of plasma membranes, 103, I18. See Conductivity

Response, general features of organic, 8; inhibitory, 45

Rhythm, in activity of living cells, 25I, 33I; in bioelectric phenomena, $330 \mathrm{ff}$.; in surfacereactions, 247,250

Salts, inorganic, action of pure solutions, I64 ff.; action on permeability, II9, I $74 \mathrm{ff}$., 35I; antagonisms between salts and anæsthetics, 2II, 352; antagonistic action of, I $55,157,1_{5} 8$, I 64 ff., I $76, \quad$ I8I ff., $35 \mathrm{I}$; balanced action, $\mathrm{I} 67 \mathrm{ff} . ;$ in cells, II 7, I 20 ff., I60; physiological action of, I 5 I ff.; sensitization by, $162,367 \mathrm{ff}$.; stimulation by, I66, 35 I ff.

Secondary stimulation, 388, 389, 392

Secretion, 94, I40, 374

Semi-permeability, $20,57,58$, I03, I09; factors in, I32, 302 . See Permeability

Sensitivity, chemical, 266; light, 266. See Irritability

Sensitization, by salt-solutions, 162, $367 \mathrm{ff}$; specific, 370 . See Anaphylaxis

Soaps, relation to protoplasmic structure, $58, \mathrm{I} 80, \mathrm{I} 85$

Specific energies, law of, 267

Specificity, 5, $30 \mathrm{ff}$. See Syntheses, specific

Square root law, 279, 282, 286

Stabilization, of protoplasmic structure, $207 \mathrm{ff}$.; of suspensions, 230, 231

Stationary systems, 48

Stimulation, bioelectric variations in, 297, 312 ff.; changes of polarization in, 275, $276 \mathrm{ff}$., 294; chemical, 266; dependence on surface changes, $269 \mathrm{ff}$., $296 \mathrm{ff}$.; electrical, 46, $285 \mathrm{ff}$; general features, 8,43 ff., 50,5 I, 60, 62 , 74,259 ff.; by light, 266; mechanical, $27 \mathrm{I}$; membrane changes in, 207 ff., 297, 302, $337 \mathrm{ff} ., \quad 346 \mathrm{ff}$. (see Permeability); by salts, I66; summation in, $277, .29$ I ff.; timerelations of, $278 \mathrm{ff}$.

Structure, protoplasmic, relation to chemical activity of living matter, 52 ff., 63. See Protoplasm

Summation, in activation of passive iron, 255 ; in stimu- lation, $277,29 \mathrm{rff}$.

Surface-activity, $82,90, \quad 187$; relation to contact potentials, $236 \mathrm{ff}$.; relation to physiological action, 187, $196 \mathrm{ff} ., 217$

Surface-films. See Films, interfacial

Surface-tension, $71,72,78,90$, I 78, I92, I98 ff., 237, 238, 249

Synapses, 272

Synchronous activity, of cells, 392

Syntheses, as basis of growth and development, 3, 4 ff., 42 ff., 54, 2I7; dependence on protoplasmic structure, $53,54,63$; electrical factors in, 402; relation to protoplasmic interfaces, 217, 218; specific, 27, 30, 31, $34 \mathrm{ff.}, 42,48,53,54,63$

Temperature-coefficients, 44; of bioelectric variations, $316,32 \mathrm{I}$, $333 \mathrm{ff}$.; of demarcation potentials, 319; of minimal duration of threshold stimulus, 286, 292; of summation-interval, 292; of protoplasmic transmissions, 34I, 342 ; of recovery of transmissivity in passive iron, 346 ; of refractory period, 292, 34 I

Transmission, in nerve and other protoplasmic systems, I52, $259 \mathrm{ff} ., 267 \mathrm{ff} ., 379 \mathrm{ff}$.; in passive 
iron, $253 \mathrm{ff} ., 260,270 \mathrm{ff}$., 298, Valence, factor in physical action 382,385 ; in plants, 374. See of ions, $91,92,93$; factor in Stimulation

Trigger action, 262

Turgor, changes resulting from stimulation, 372 ff. physiological action of iunt, (1), 167 ff., 175

Visual purple, 260

Vitalism, 2

Vitamines, $260 \mathrm{n}$.

Ultra-filtration, I07, I 35

Ultramicroscopy, $5^{8}$

Ultraviolet rays, activation by, 355,363

Water-immiscibility, of protoplasm, 22, 57

Y cast, 27 


North Carolina Sta

QH331 .L55

PROTOP55

PROTOPLASMIC ACTION AND NERVOUS ACTION

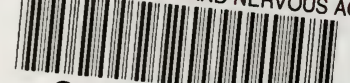

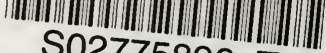

S02775896 T 